
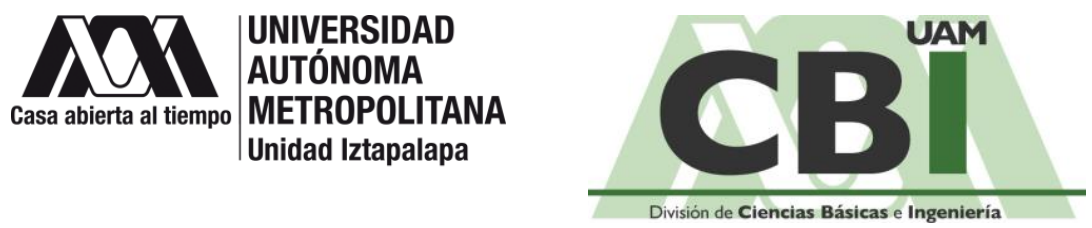

\title{
THESIS
}

\section{To obtain a}

Philosophiæ doctor in Information Sciences and Technology

Granted by : Metropolitan Autonomous University Campus Iztapalapa

\begin{tabular}{c}
\hline Presented by: \\
Omar Alejandro Olvera-Guerrero \\
Boiling water reactor stability analysis based on non-linear techniques \\
\hline \hline
\end{tabular}

JURY:

Presidente: Gilberto Espinosa Paredes

Secretario: Ángel Tomás Aljama Corrales

Vocal: Alejandro Núñez Carrera

Vocal: Miguel Ceceñas Falcón

Vocal: Juan Carlos Sánchez García

Thesis advisors:

Dr. Prieto-Guerrero Alfonso

Dr. Paredes-Espinosa Gilberto

Mexico City - Mexico

Grade Exam Date: January 31 2020,

UAM-I, Classroom: EP101 at 16:00hrs. 


\section{Acknowledgments}

I would like to thank Dr. Prieto-Guerrero Alfonso and Dr. Espinosa-Paredes Gilberto for allowing me to participate in their project associated to boiling water reactor instability. I also thank the department of Electrical Engineering for accepting me as a new student of their graduate program on Sciences and Technologies of Information. I thank my family for supporting me along the difficult journey to attain a PhD. I thank the Mexican national council of science and technology (Conacyt) for providing the financial resources to fund my thesis project through a doctoral scholarship. My experience thus far has been like a wheel of fortune, a series of ups and downs and I would like to thank and to show my appreciation to those individuals that supported me during hardships these past few years. 


\begin{abstract}
The most common indicator currently employed in practice to assess boiling water reactor (BWR) instability, due to density wave oscillation (DWO) is the Decay Ratio (DR), an easy to grasp index that is regularly calculated from an estimate of the impulse response function of the reactor core, such impulse response appraisal is most of the time provided by an autoregressive (AR) modeling of the reactor core. The DR is the output of most stability monitoring systems available in the market. However, it is known that BWRs are intricate systems that may exhibit complex dynamics during instability that cannot be captured by the DR alone. Besides, AR models require linear and stationary signals to grant reliable models. Recorded BWR signals are not linear and are not stationary. Therefore, it is necessary to reignite BWR stability studies to develop more suitable stability methodologies and indicators capable of accommodating the complex nature of unstable BWR signals. To address this issue, the work presented in this thesis is related to the study of non linear signal processing methodologies to assess the stability of a BWR, due to DWO.
\end{abstract}

This thesis is divided in eight chapters. The first one introduces the various BWR instability types that have been observed in practice, it also introduces the DR definition. The second chapter introduces a state of the art of how BWR instability has been studied in the last three decades. The third chapter introduces the Empirical mode decomposition (EMD), a non-linear filter that accommodates non-stationary and non-linear behavior from real world signals. The EMD is the backbone of most of the BWR instability proposals given in this work. In the fourth chapter, a reduced order model (ROM) is studied, such ROM represents qualitatively the chaotic dynamic behavior of a BWR system during instability. The fifth chapter introduces the first non-linear instability indicator: The Shannon Entropy (SE) and its associated tests with BWR recordings is discussed in this chapter. The sixth chapter introduces the second non-linear instability indicator, the Sample Entropy (SampEn) and its experiments are discussed in this chapter too. Chapter seven discusses the third and final (and most powerful) non-linear instability indicator proposal: The Higuchi Fractal Dimension (HFD) and its associated experiments with artificial and real BWR signals are shown and discussed in this chapter. In Chapter eight, we introduce a novel and practical BWR instability monitor with decision rules based on the HFD for real time application. Final conclusions of the work performed in this thesis are given in chapter nine. 


\section{Contributions}

\section{Paper I}

Olvera-Guerrero, O.A., Prieto-Guerrero, A., \& Espinosa-Paredes, G. (2017). Decay Ratio estimation in BWRs based on the improved complete ensemble empirical mode decomposition with adaptive noise. Annals of Nuclear Energy, 102, 280-296.

\section{Paper II}

Olvera-Guerrero, O.A., Prieto-Guerrero, A., Espinosa-Paredes, G. (2017). Non-linear boiling water reactor stability with Shannon Entropy. Annals of Nuclear Energy, 108, 1-9.

\section{Paper III}

Olvera-Guerrero, O.A., Prieto-Guerrero, A., \& Espinosa-Paredes, G. (2017). Non-linear stability analysis of Real Signals from Nuclear Power Plants (Boiling Water Reactors) Based on Noise Assisted Empirical Mode Decomposition Variants and the Shannon Entropy. Entropy, 19(7), 359.

\section{Paper IV}

Olvera-Guerrero, O.A., Prieto-Guerrero, A., \& Espinosa-Paredes, G. (2018). A Novel Nonlinear BWR stability Indicator Based on the Sample Entropy. Science and Technology of Nuclear Installations, 2018.

\section{Paper V}

Olvera-Guerrero, O.A., Prieto-Guerrero, A., \& Espinosa-Paredes, G. (2020). A non-linear stability for Boiling Water Reactors. Annals of Nuclear Energy, 135, 106983. 


\section{Contenido}

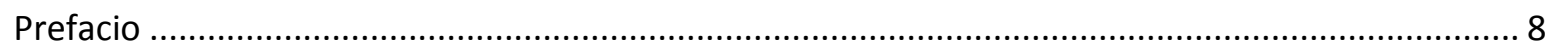

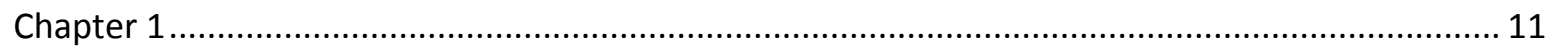

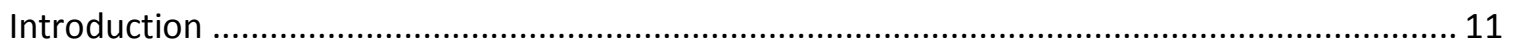

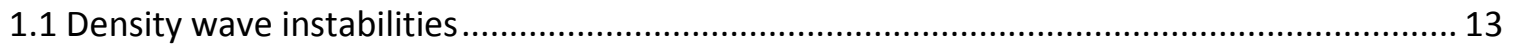

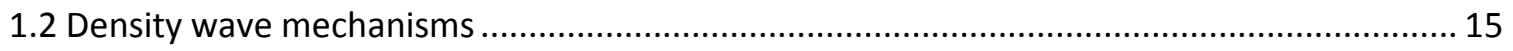

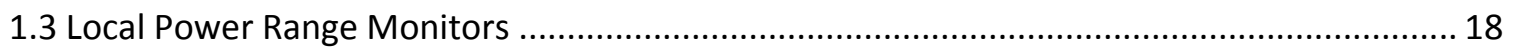

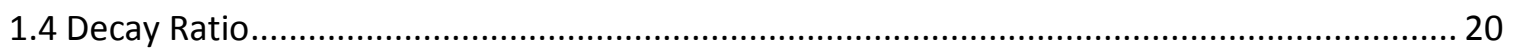

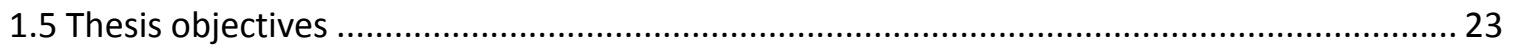

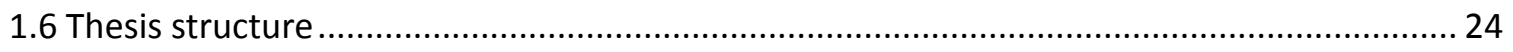

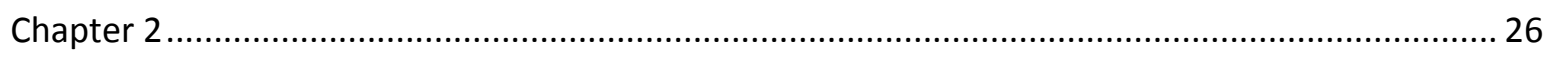

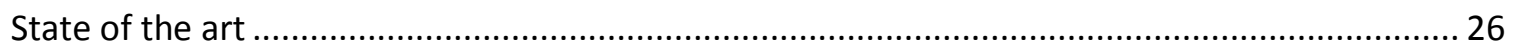

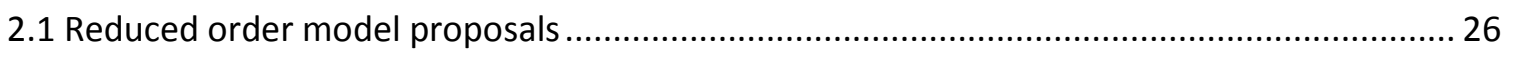

2.2 Autoregressive modeling for decay ratio estimation .................................................... 27

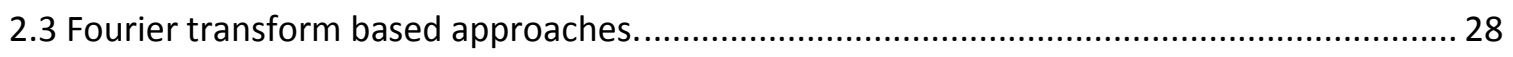

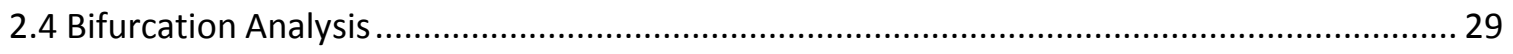

2.5 Wavelet based methodologies for DR estimation ........................................................ 29

2.6 Empirical mode decomposition based methodologies for DR estimation ............................ 30

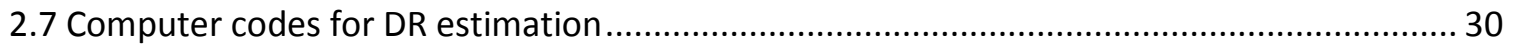

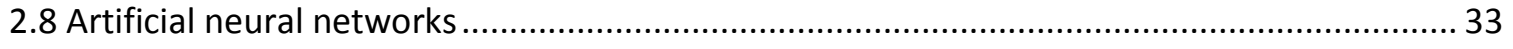

2.9 Time line of techniques proposed to assess BWR stability over the past 40 years ................ 34

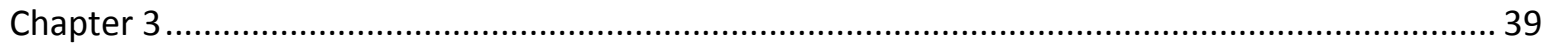

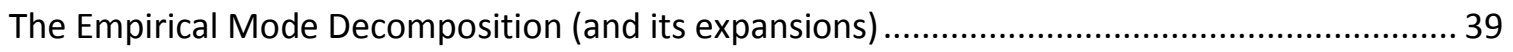

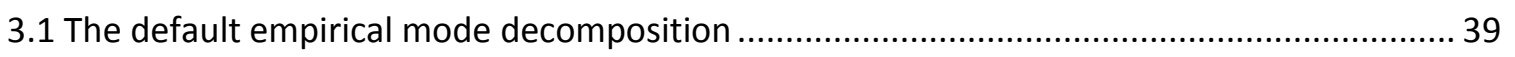

3.2 The ensemble empirical mode decomposition ............................................................... 44 
3.3 The complete ensemble empirical mode decomposition with assisted noise ....................... 46

3.4 The improved complete ensemble empirical mode decomposition with assisted noise....... 49

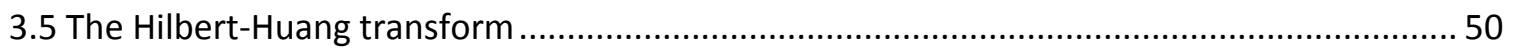

3.6 The noise assisted multivariate empirical mode decomposition........................................... 56

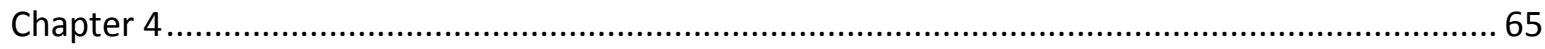

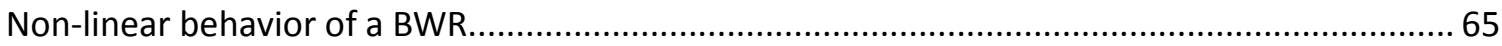

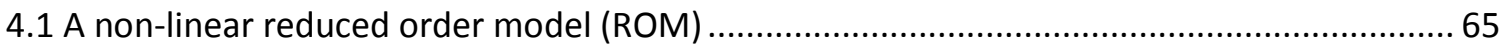

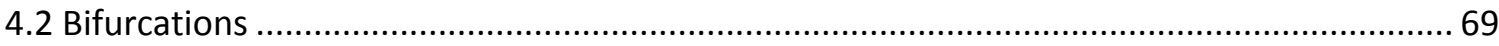

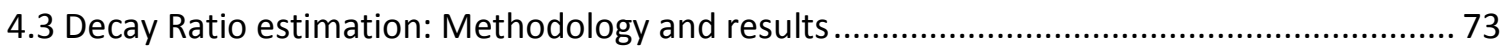

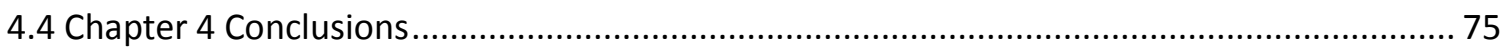

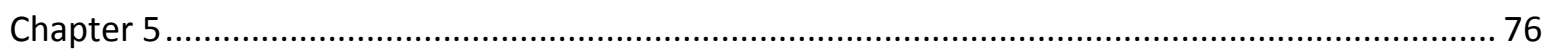

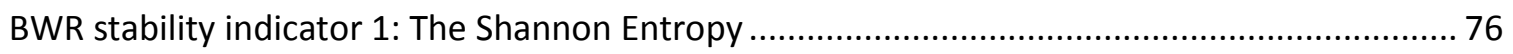

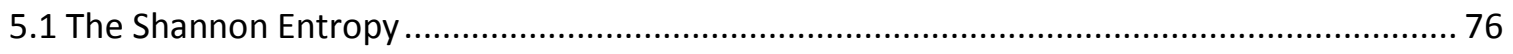

5.2 BWR stability monitor based on the Shannon Entropy for artificial signals ......................... 78

5.3 Non-linear stability analysis of BWR real signals based on noise assisted empirical mode

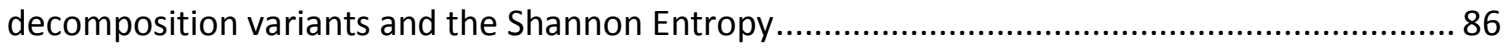

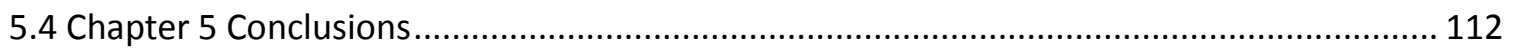

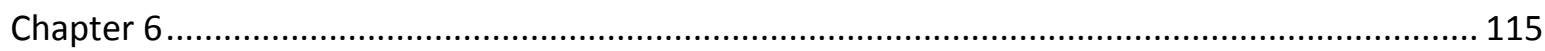

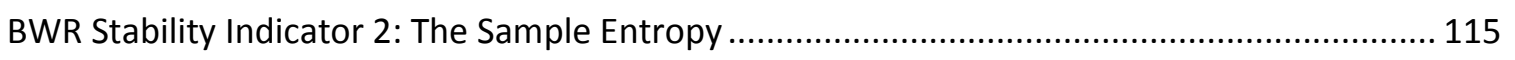

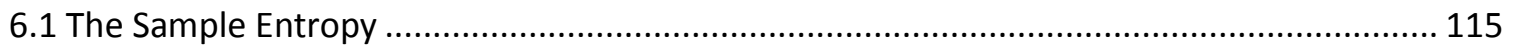

6.2 BWR stability methodology based on the SampEn and the NA-MEMD ............................. 118

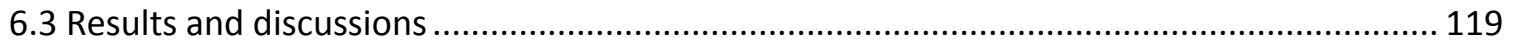

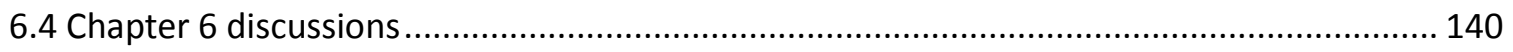

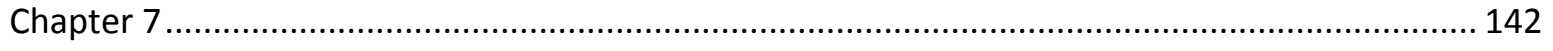

BWR stability indicator 3: The Higuchi fractal dimension .................................................. 142 


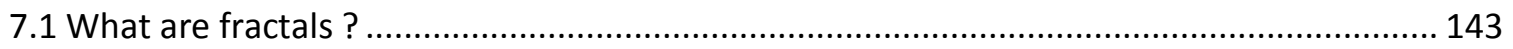

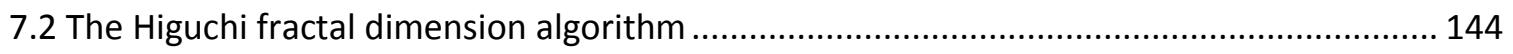

7.3 How to select an adequate kmax free parameter value? ............................................... 145

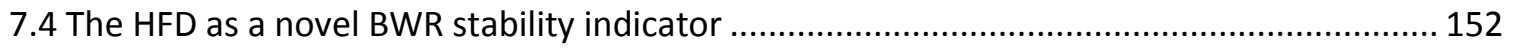

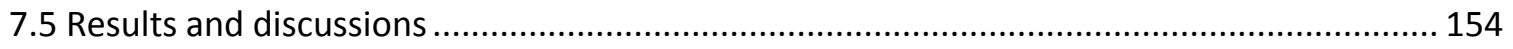

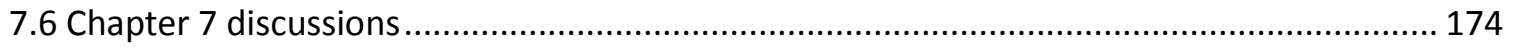

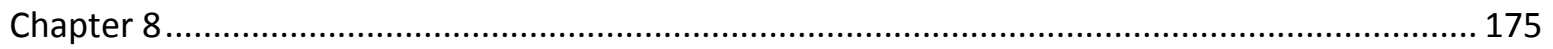

A non-linear stability monitor for boiling water reactors based on the Higuchi fractal dimension

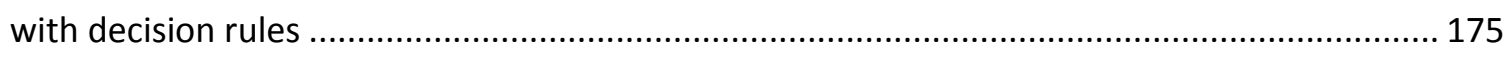

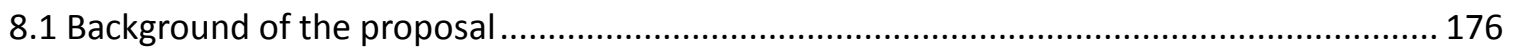

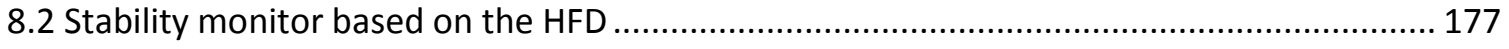

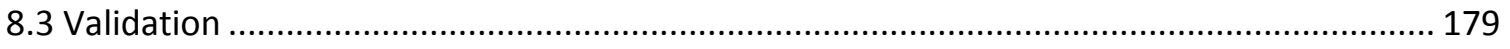

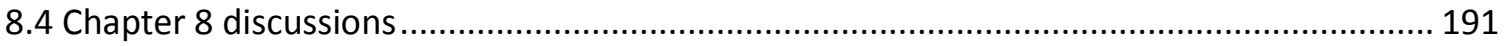

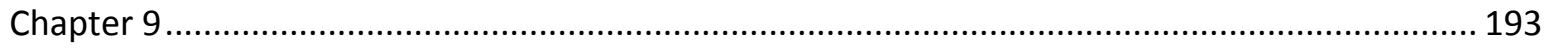

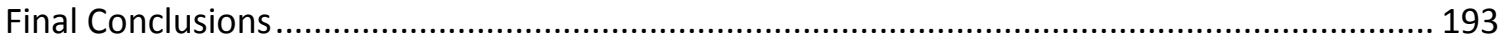

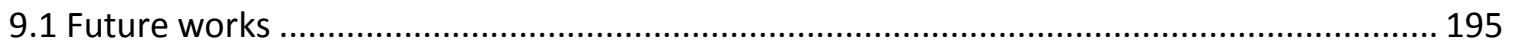

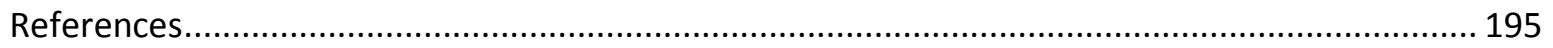




\section{Prefacio}

La elaboración de la presente tesis surge a raíz del interés de desarrollar técnicas para estudiar la estabilidad de reactores nucleares de agua en ebullición (BWR, por sus siglas en inglés). Nos interesa entender a través de las señales medidas de centrales nucleares reales, qué es lo que ocurre desde el punto de vista fenomenológico cuando ocurre una inestabilidad de potencia en reactores muy específicos que son del tipo BWR como el de Laguna Verde. Los BWR pueden presentar problemas de inestabilidad de potencia de tipo divergentes; es decir, que la potencia va creciendo en forma divergente hasta que llega a los puntos donde el reactor pueda apagarse en forma automática (bajo el supuesto de que la planta cuenta con tales diseños).

A través de los años se han hecho esfuerzos internacionales para contar con detectores de inestabilidad, y para tener una idea clara en qué consisten se puede hacer una analogía con una alarma sísmica. "Supongan que con alguna metodología se puede establecer que el inicio de un temblor puede ocurrir un minuto o un minuto y medio antes de lo que actualmente se avisa". En el pasado se han desarrollado métodos que permiten establecer con mucho tiempo de anticipación y poca información, que puede ocurrir una inestabilidad en un reactor nuclear. Lo anterior redunda en una mayor seguridad de la planta, pero además en que el operador cuenta con más tiempo para poder tomar acciones pertinentes. El problema actual de muchas metodologías de estabilidad tiene que ver con sus métodos de construcción. La gran mayoría de métodos empleados para estudiar la estabilidad de BWRs es que son herramientas lineales que no pueden medir toda la complejidad de un "sistema dinámico" como un reactor nuclear. Tales herramientas lineales asumen que el reactor se comporta bajo inestabilidades como un sistema de segundo orden o como un oscilador armónico. Lo cual es una suposición demasiado simple y distante de la realidad. Además, se ha observado que los BWRs bajo inestabilidad pueden llegar a presentar comportamiento "caótico". El "caos" no puede ser detectado con las herramientas existentes.

Nuestro objetivo es desarrollar metodologías de estabilidad haciendo uso de métodos nolineales, las cuales son técnicas que se han desarrollado para estudiar la naturaleza de sistemas dinámicos complejos y/o caóticos como un BWR. En la literatura se ha explorado poco el estudio de estabilidad de BWRs con herramientas no-lineales. Por lo tanto, nos encontramos como investigadores en suelo fértil. Las siguientes secciones están escritas en inglés, para que cualquier persona perteneciente a nuestra comunidad de científicos pueda hacer uso de ella en algún futuro. 
Los lectores encontraran lo siguiente dentro de esta tesis:

El Capítulo 1 está dedicado a introducir el tipo de inestabilidad BWR más comúnmente observado en las centrales nucleares comerciales, que es: la inestabilidad de la onda de densidad. Este capítulo también proporciona un resumen de los eventos de inestabilidad de BWR informados y de los instrumentos dentro del núcleo que se utilizan para evaluar la estabilidad de BWRs por razones de seguridad. Nuestros objetivos también se encuentran en este capítulo.

El Capítulo 2 presenta el estado del arte de algunas obras que se han publicado en los últimos 40 años para analizar la inestabilidad de BWR. Además, las propiedades lineales o no lineales de los métodos proporcionados en esta sección se dan al final de esta sección, junto con algunos de sus obstáculos para la implementación en tiempo real.

El Capítulo 3 presenta la descomposición de modos empírico (EMD), un filtro no lineal para descomponer señales no estacionarias que provienen de sistemas no lineales en un esquema de resolución múltiple. Este capítulo aborda las expansiones de EMD que se inspiraron en el EMD estándar para mejorar la descomposición de datos complejos. En este capítulo, también presentamos una expansión multivariada de EMD para analizar datos multicanal.

El Capítulo 4 presenta un modelo simple pero potente de orden reducido (ROM) de un BWR. La ROM ayuda a comprender el comportamiento dinámico de un BWR bajo inestabilidad de onda de densidad. Realizamos algunos experimentos con la ROM para probar las limitaciones del DR estándar cuando se trata de evaluar la inestabilidad de BWR. Las limitaciones del DR expuestas en este capítulo abren la puerta para estudiar la inestabilidad de BWR (bajo onda de densidad) con otras herramientas más sofisticadas.

El Capítulo 5 presenta la primera medición para evaluar la inestabilidad de BWR (bajo onda de densidad). La entropía de Shannon (SE), la SE es una medida no lineal de la complejidad de una variable aleatoria. La Entropía de Shannon se utiliza con frecuencia en telecomunicaciones. No obstante, aquí la aplicamos para evaluar la inestabilidad de los BWR mediante las grabaciones de los LPRM. Aquí desarrollamos las primeras metodologías de inestabilidad BWR no lineal en este capítulo con el SE en combinación con expansiones EMD.

El Capítulo 6 analiza la segunda medición de estabilidad (una segunda propuesta) para evaluar la inestabilidad de los BWRs. La nueva medida es la Entropía de muestra (SampEn). El SampEn es una medida que se utiliza para evaluar la complejidad de señales fisiológicas, para diagnosticar "estados" enfermos. Aquí se aplica para separar los estados estables de BWR de los inestables. SampEn es una fórmula informática sofisticada para medir la complejidad de las series de tiempo, dicha fórmula se puede implementar en 
tiempo real. El SampEn es un indicador muy sofisticado, más que la Entropía de Shannon que se introduce en el Capítulo 5. Elaboramos una metodología de estabilidad de BWRs basada en SampEn en combinación con una expansión EMD multi variable.

El Capítulo 7 presenta la tercera propuesta de estabilidad (y la más poderosa), la dimensión Fractal Higuchi (HFD), una fórmula que mide la dimensión fractal de las series de tiempo. La dimensión fractal es un índice de rugosidad de una serie temporal. EL HFD proporciona estimaciones confiables de dimensiones fractales con pocos puntos de datos (esto es útil cuando hay pocos datos disponibles). El HFD solo necesita un parámetro de entrada simple a priori para su funcionamiento. Elaboramos una metodología de estabilidad BWR basada en HFD en combinación con una expansión EMD multi variable.

El Capítulo 8 discute las limitaciones de los tres indicadores estudiados de los últimos 3 capítulos. El indicador más fuerte entre los tres estudiados se elige para construir un monitor de estabilidad BWR robusto y práctico para la detección de oscilación DW con reglas de decisión. El indicador elegido es la potente dimensión Fractal de Higuchi (HFD).

En el Capítulo 9 se exponen nuestras conclusiones y se dan sugerencias de futuros proyectos que podrían surgir a raíz de lo expuesto dentro de esta tesis. 


\section{Chapter 1}

\section{Introduction}

Today, there are 78 nuclear boiling water reactors (BWRs) in the world dedicated to the generation of electricity. BWRs make a significant contribution to the production of global electric power and to date, BWRs are the simplest energy system to transform fission energy into electrical energy, due to the direct cycle to turbine with dry saturated steam. Nonetheless, there are many fundamental aspects related to their operation that under certain conditions, may induce BWR malfunctioning and affect its stability. The problem of the stability of the BWR has been a subject of significant scientific and technological work during more than four decades dedicated to its study.

Instability events may occur during start up or during transients to change the region of operation of the reactor. Figure 1 shows the example of a typical power-flow map diagram of a nuclear power plant (NPP), the map shows the regions where the reactor should not be operated (red colored region) for reasons of stability, those ones where the BWR can be operated only under supervision (brown colored region) and finally, the diagram shows the regions of stable reactor operation ( regions where the core flow is high). Nowadays, there is a tendency to design reactor of higher power. In addition, refinement of fuel elements has encouraged the introduction of increasingly efficient fuels that allow the plant to operate at increasingly high power levels. Such a power increase induces a higher reactivity feedback and a decrease in response time, resulting in a lower BWR stability range when the plant operates at a low mass flow and at high nominal power. Another current trend is to increase the size of the core, which causes a weaker coupling in the neutron field which increases the susceptibility of the reactor to experiencing unstable oscillations. In summary, all current tendencies related to reactor design enhance the regions where the reactor should not be operated (reactor operation at low flow and high power). 


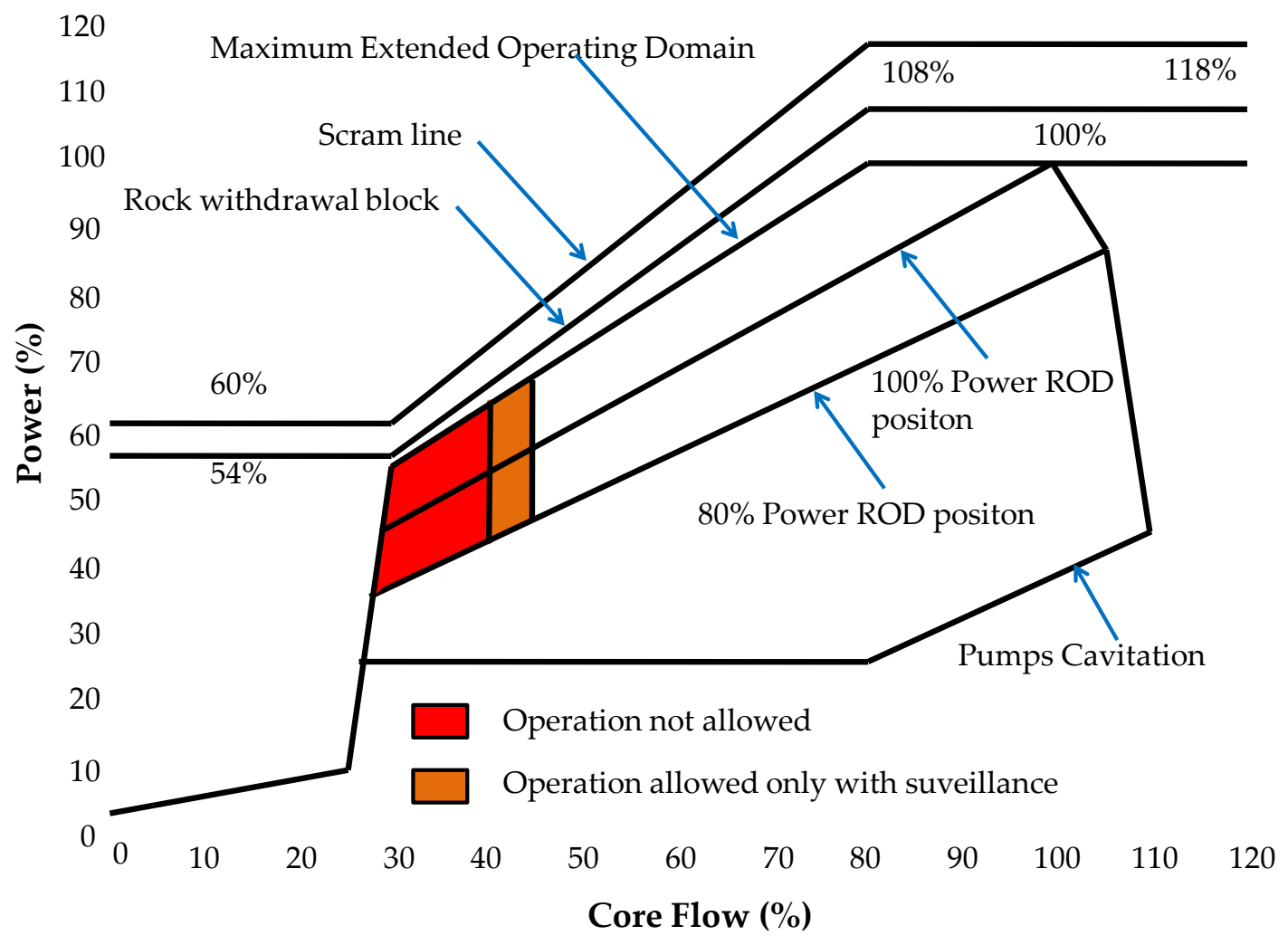

Figure 1. Typical Power-Flow map of a BWR.

Events of instability have already occurred in the past in commercial BWRs, such as the Laguna Verde Nuclear Power Plant (Gonzalez et al., 1995 and Farawila et al., 1996). Some cases of instability occurred inadvertently, while others were intentionally provoked for experimental purposes (Verdú et al., 2001). Periodic oscillations in the neutron flux were observed during these instability events via the electronic instrumentation of the reactor. After the first events of instability occurred, the corresponding authorities (regulatory commissions) requested the development of research projects to study the mechanisms involved in reactor stability to:

1. Study the stability margins of the plant under normal operating conditions and in unusual conditions.

2. Predict reactor transients in an event of instability.

3. Develop measures to prevent and mitigate the consequences of an event of instability. 


\subsection{Density wave instabilities}

BWRs have complex responses that may result in various instability types, each type is characterized by a particular oscillating mode. Table 1 shows a summary of reported BWR instability events that have been observed in the past (Reproduced from D'Auria., 2008).

Table 1: Summary of reported BWR Instability Events.

\begin{tabular}{|c|c|c|}
\hline Date & NPP, Country, Manufacturer & Event \\
\hline $30 / 06 / 1982$ & Caorso, Italy, GE (General Electric) & $\begin{array}{l}\text { It occurred during startup of the } \\
\text { reactor. The reactor thermal power } \\
\text { was } 53.5 \% \text { and the recirculation flow } \\
38 \% \text {. The operating point of the } \\
\text { reactor entered the unstable region, } \\
\text { the oscillations diverged and the } \\
\text { reactor scrammed on high-high } \\
\text { APRM signal (120\% power). }\end{array}$ \\
\hline $13 / 01 / 1984$ & Caorso, Italy, GE & $\begin{array}{l}\text { The second event occured after trio of } \\
\text { a recirculation pump and following } \\
\text { loss of some preheater trains. The } \\
\text { cold feed water and the strongly } \\
\text { peaked axial power distribution led to } \\
\text { neutron flux oscillation. }\end{array}$ \\
\hline $23 / 02 / 1987$ & TVO-I, Finland, ABB Atom & $\begin{array}{l}\text { A power oscillaiton event took place } \\
\text { when the plant was brought back to } \\
\text { power after a short shutdown period. } \\
\text { The reactor power was about } 60 \% \\
\text { with a recirculation flow of } 30 \% \\
\text { when increased APRM signal } \\
\text { oscillations started to appear. }\end{array}$ \\
\hline $09 / 03 / 1988$ & LaSalle 2, USA, GE & $\begin{array}{l}\text { Underwent a dual recirculation pump } \\
\text { trip following which the unit } \\
\text { experienced excessive neutron flux } \\
\text { oscillations while it was in natural } \\
\text { circulation. The resulting perturbation } \\
\text { on the switches for anticipated } \\
\text { transient without scram resulted in a } \\
\text { trip of both recirculation pumps. }\end{array}$ \\
\hline $15 / 01 / 1989$ & Forsmark 1, Sweden, ABB Atom & $\begin{array}{l}\text { After having carried out tests in } \\
\text { several of the selected operating } \\
\text { points, the stability boundary was } \\
\text { even close. Moving from one point to } \\
\text { another, the operator chose to switch } \\
\text { from the pump speed control mode to } \\
\text { the power control mode. This was } \\
\text { done at } 71 \% \text { power and } 4700 \mathrm{~kg} / \mathrm{s} \text {. A } \\
\text { remaining control mismatch led to a } \\
\text { small decrease in core flow. The } \\
\text { combined effect of the power control } \\
\text { system, caused power oscillation with } \\
\text { an amplitude that increased to about } \\
20 \% \text { after } 20 \mathrm{~s} \text { enough the initiate a } \\
\text { pump run-down. }\end{array}$ \\
\hline $26 / 10 / 1989$ & Ringhals 1 , Sweden, ABB Atom & $\begin{array}{l}\text { In } 1989 \text { these NPP was starting up for } \\
\text { a new cycle, power oscillations were } \\
\text { observed in the core. During the } \\
\text { oscillations, several LPRMs gave a } \\
\text { high level alarm, indicating that the }\end{array}$ \\
\hline
\end{tabular}




\begin{tabular}{|c|c|c|}
\hline & & $\begin{array}{l}\text { local power in those positions had } \\
\text { exceeded } 118 \% \text {. The core was } \\
\text { stabilized about } 30 \mathrm{~s} \text { after the partial } \\
\text { scram. }\end{array}$ \\
\hline $08 / 01 / 1990$ & Oskarshamn 2, Sweden, ABB Atom & $\begin{array}{l}\text { A planned power reduction from } \\
106 \% \text { to } 65 \% \text { was performed. The } \\
\text { power was reduced by reducing pump } \\
\text { speed to a minimum. After about } 1 \\
\text { minute, power oscillations had } \\
\text { developed, having peak-to-peak } \\
\text { amplitudes of }+/-10 \% \text {. The APRM } \\
\text { signals showed the oscillations to be } \\
\text { in phase. A manual partial scram was } \\
\text { performed which completely } \\
\text { quenched the oscillations. }\end{array}$ \\
\hline $29 / 01 / 1991$ & Cofrentes, Spain, GE & $\begin{array}{l}\text { Oscillations appeared after the } \\
\text { operator withdraw control rods and } \\
\text { reduced core flow in order to transfer } \\
\text { the recirculation pumps from low to } \\
\text { high speed. }\end{array}$ \\
\hline $03 / 07 / 1991$ & Isar 1, Germany, Siamens & $\begin{array}{l}\text { The Isar NPP had a trip of four } \\
\text { internal recirculation pumps, due to a } \\
\text { reduction on seal water flow to the } 8 \\
\text { recirculation pumps. The power } \\
\text { reduction due to the control rod } \\
\text { insertion proved to be too slow to } \\
\text { prevent the reactor from entering the } \\
\text { unstable region of the power-flow } \\
\text { map. neutron flux oscillations with } \\
\text { increasing amplitude appeared at } \\
\text { about } 50 \% \text { power and } 30 \% \text { core flow. } \\
\text { When the oscillations reached peak- } \\
\text { to-peak amplitude of } 30 \% \\
\text { corresponding to a peak power of } \\
65.5 \% \text { at } 30 \% \text { flow, the reactor } \\
\text { scrammed on the underlayed setpoint } \\
\text { of neutron flux to core flow ratio. } \\
\text { This occurred } 44 \text { seconds after the trip } \\
\text { of the four recirculation pumps. }\end{array}$ \\
\hline $15 / 08 / 1992$ & WNP 2, USA, GE & $\begin{array}{l}\text { The WNP } 2 \text { experienced power } \\
\text { oscillations during startup. The event } \\
\text { occurred early in cycle } 8 \text { operation. } \\
\text { Upon recognizing the power } \\
\text { oscillations, the operators manually } \\
\text { initiated a reactor scram. Post event } \\
\text { review indicated that the oscillations } \\
\text { were in-phase and had grown to peak- } \\
\text { to-peak amplitude of about } 25 \% \text { of } \\
\text { rated power. }\end{array}$ \\
\hline $09 / 07 / 1993$ & Perry, USA, GE & Entry into a region of core instability \\
\hline $01 / 1995$ & Laguna Verde 1, Mexico, GE & $\begin{array}{l}\text { During startup of the reactor, at } 34 \% \\
\text { power, the operator was waiting to } \\
\text { increase pump recirculation speed. } \\
\text { Power was increased to } 37 \% \text { by } \\
\text { control rod withdrawal and closure of } \\
\text { the control valves of the pumps was } \\
\text { initiated, thus leading to power } \\
\text { reduction. During these operations the } \\
\text { operator observed power oscillations } \\
\text { with a tendency to diverged and he }\end{array}$ \\
\hline
\end{tabular}




\begin{tabular}{|l|l|l|}
\hline & & $\begin{array}{l}\text { stopped the closure of the valves. At } \\
\text { this time the peak-to-peak amplitude } \\
\text { in the oscillations was 6\%. }\end{array}$ \\
\hline $17 / 07 / 1996$ & Forsmark 1, Sweden, ABB Atom & $\begin{array}{l}\text { Local oscillations due to a bad seated } \\
\text { fuel assembly. }\end{array}$ \\
\hline $08 / 02 / 1998$ & Oskarshamn 3, Sweden, ABB Atom & $\begin{array}{l}\text { Power oscillations due to a bad } \\
\text { combination of core design and } \\
\text { control-rod pattern during start up }\end{array}$ \\
\hline $25 / 02 / 1999$ & Oskarshamn 2, Sweden & $\begin{array}{l}\text { Power oscillations after a turbine trip } \\
\text { with pump runback }\end{array}$ \\
\hline
\end{tabular}

In this thesis we will only focus on the most common instability type that has been observed in commercial BWR reactors. Such instability is called by the name: Density wave Instability (DW). We will now focus on the mechanisms that generate them.

\subsection{Density wave mechanisms}

A seasonal reduction of inlet flow in a heated channel increases the rate of enthalpy rise, thereby reducing the average density. This disturbance affects the pressure drop and the heat transfer behavior. For certain combinations of geometrical arrangement, operating conditions, and boundary conditions, the perturbation can acquire a $180^{\circ}$ out-of-phase pressure fluctuation at the exit, immediately transmitted to the inlet flow rate and become self sustained (Stenning and Veziroglu, 1965). For boiling systems, the oscillations are due to multiple regenerative feedbacks between the flow rate, vapor generation rate, and pressure drop (Neal et al., 1967). Since transportation delays are of paramount importance for the stability of the system (Bouré, 1966; Bouré and Ihaila, 1967), the phrase "time-delay oscillations" has also been used. More practically, these are low frequency oscillations (centered around $0.5 \mathrm{~Hz}$ ) in which the period is approximately one to two times the time required for a fluid particle to travel through the channel. Accordingly, density wave oscillations and density effect mechanism are also used to describe the phenomena. DW instabilities are the most common instabilities observed in commercial NPPs (MarchLeuba, 1992). Figure 2 illustrates the mechanisms that generate DWs. 


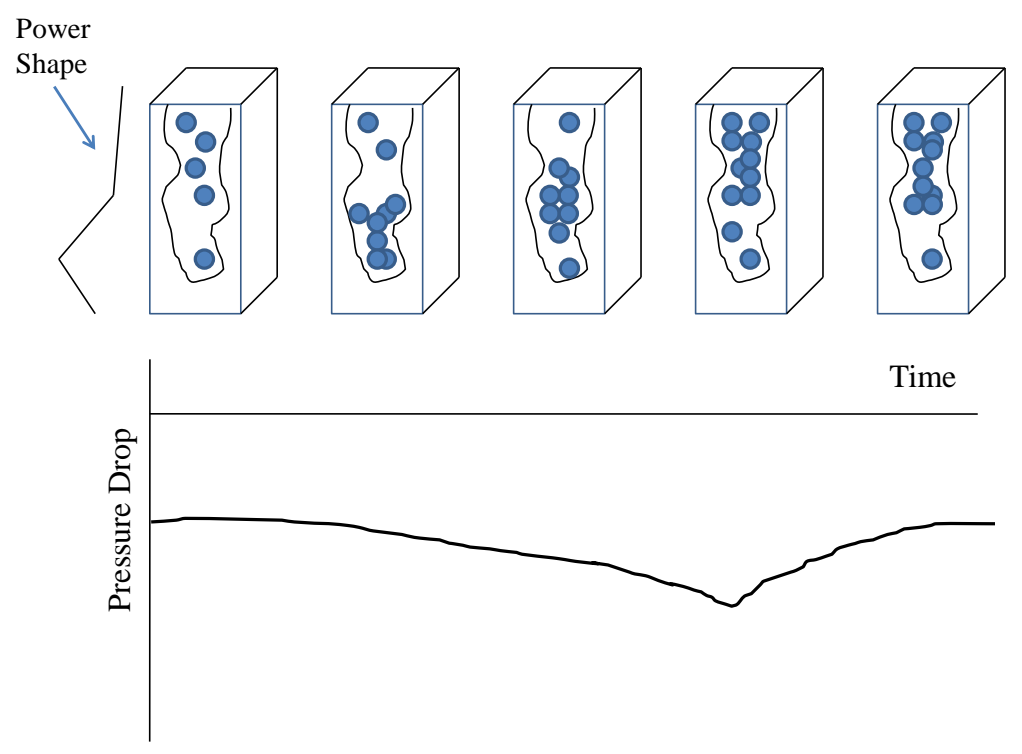

Figure 2. Density wave mechanism introduces a lag. The effect of a power pulse is seen a few seconds later in the channel pressure drop due to void propagation delay.

There are two kinds of DW oscillating modes: in-phase (global or core-wide) oscillations, and out-of-phase (regional) oscillations. In-phase oscillations are caused by the lag introduced into the thermal-hydraulic system by the finite speed of propagation of density perturbation (Lahey and Podoswski, 1989). At high-core void fractions and low flow conditions, the feedback becomes so strong that it induces frequency oscillations close to $0.5 \mathrm{~Hz}$. When this feedback increases, the oscillation becomes more pronounced, and oscillatory instability is reached. The term out-of-phase oscillation is applied to those instabilities in which different reactor core zones show a considerable phase shift (up to $\left.180^{\circ}\right)$ in neutron flux oscillation. It has been shown that stability depends on several variables such as control rod patterns, void fraction, burnup, inlet mass flow, among others. Figure 3 shows an example of an in-phase DW instability event whereas Figure 4 shows an example of an out-of-phase instability event (Please refer to Prieto-Guerrero et al., 2015 to learn the details about Figures 3 and 4). DW instabilities can be studied through the recordings of Local Power Range Monitors (LPRMs) located within the reactor vessel or core. In the following subsection, we will provide more information about how LPRMs are constructed, how they are used and how they are placed within the core. The average of various LPRMs of a particular core region is known as Average Power Range Monitor (APRM). APRMs are used for security purposes only (to trigger the emergency shutdown systems of a BWR, commonly referred to as SCRAM or Kill switches). In practice BWR stability appraisal is achieved only through APRM observation (In the estimate of many 
researchers in the field, a mistake due to the fact that Out-of-phase oscillations might not be adequately detected by APRM circuits if the phase shift is strong).

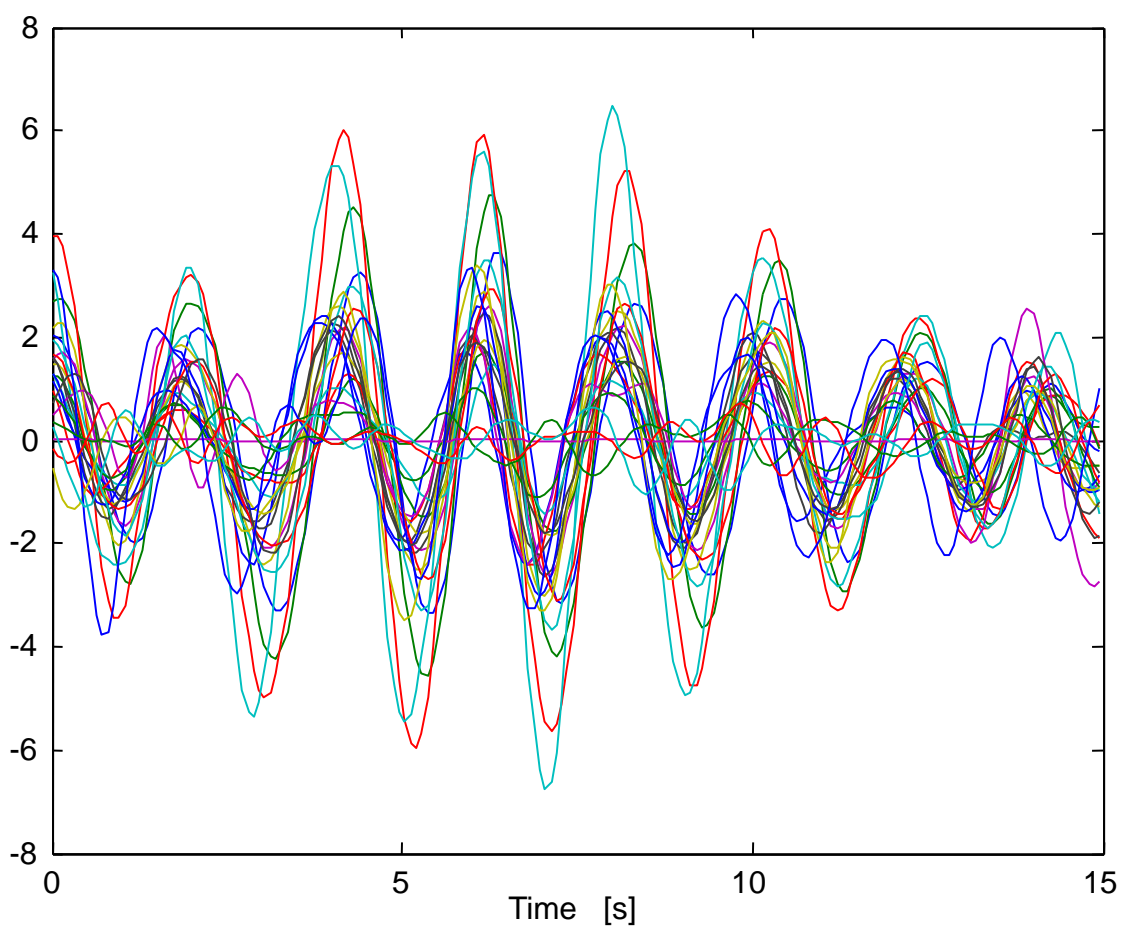

Figure 3. Example of an In-phase instability event (no phase shift observed). 


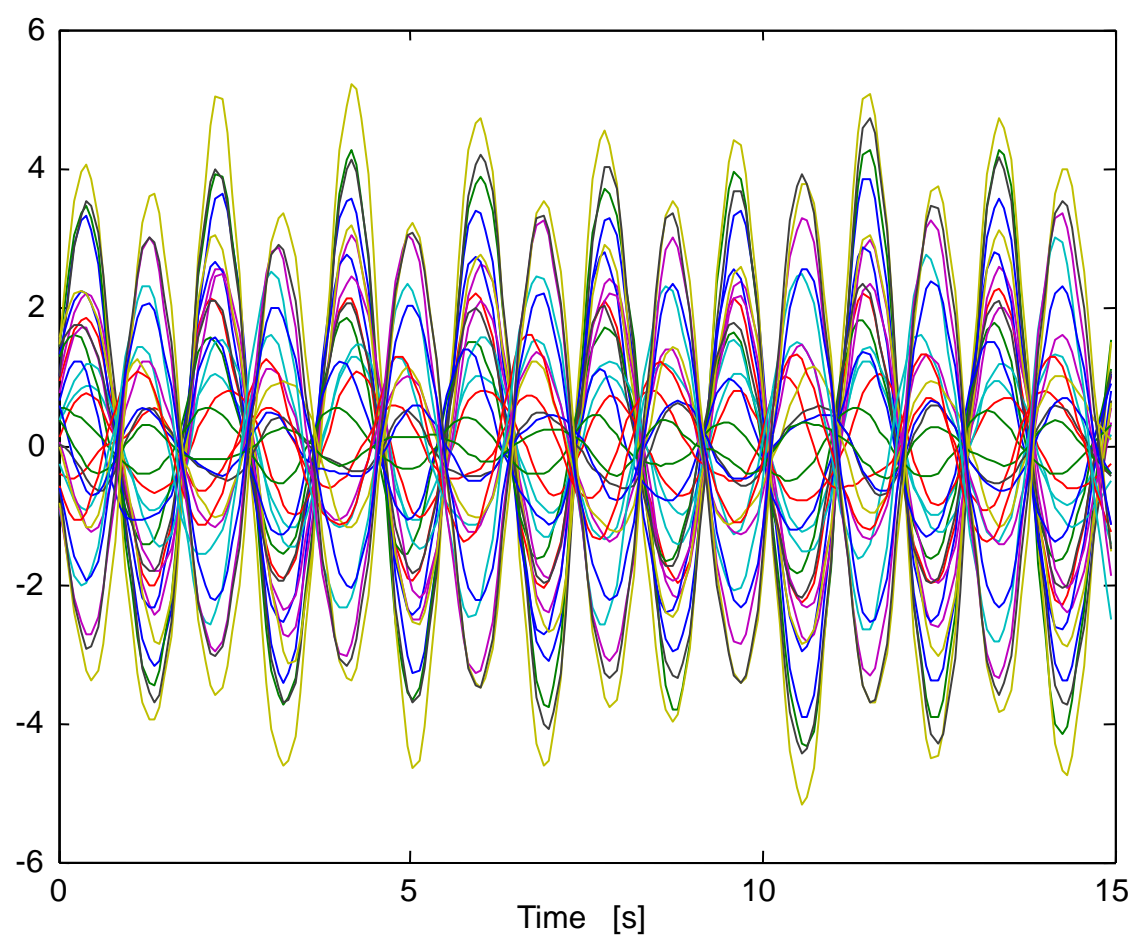

Figure 4. Example of an out-of-phase instability event (phase shift appears to be strong).

\subsection{Local Power Range Monitors}

The BWR reactor has an unconventional instrumentation system for neutron flow monitoring (NMS). In general terms the system monitors the reactor power and generates automatic action signals for core protection. The signals studied in this thesis are recorded by the local power range monitors (LPRM). LPRM detectors are fixed fission chambers inside the core, which receive high voltage and DC current. In fission chambers, nuclear reactions are carried out with the neutrons generated by fissions taking place inside the reactor core. The alfa particles are detected by the LPRMs that generate an electric pulse which is processed with amplifiers. Such flux amplifiers convert the current signal into an analog voltage signal. There is an amplifier for each LPRM detector. The gain of the amplifier can be adjusted based on the depletion of the uranium within the detector. The amplifier output is calibrated to give readings in units of Watts $/ \mathrm{cm}^{2}$ (heat flow per unit area). The LPRM signals are fed to the Average Power Range Monitors (APRMs), where the average of the flow signals is determined to monitor the overall power of the core. LPRM signals are also used to control the reactor Protection System (RPS) for reactor safety and protection.

The LPRMs are placed vertically within the core, separated from each other by a distance along the core, as shown by Figure 5. The first LPRM is located at a distance above the 
bottom of the active fuel (Bottom Active Fuel, BAF). From the bottom up, the detectors are designated with the letters $\mathrm{A}, \mathrm{B}, \mathrm{C}$ and $\mathrm{D}$. The cable of each detector terminates below the reactor vessel that attaches to the connector. There are coaxial cables that transmit the signal from the reactor, through the primary containment, to the monitoring cabinet.

The LPRMs are placed in levels that are distributed in the radial direction of the core as shown in Figure 6. Figure 6 shows in red a total of 36 LPRMs of a reactor floor or level. The reactor of the Mexican NPP of Laguna Verde has a total of 4 levels, of 24 LPRMs each. That make a total of 96 LPRMs that are fed to 4 APRM monitors, each APRM is the average of the 24 LPRMs of a reactor level. The LV APRMs are enumerated for each level as: APRM A, B, C and D. In this work, we study the LPRM recordings of three BWRs. The Forsmark and Ringhals reactors located in Sweden and the LV reactor located in southern Mexico. In this work, we focus on the analysis of LPRMs due to the fact that a strong outof-phase DW event might ruin APRM monitoring (only if the dephasing among the LPRM signals that are used to compute the average is too strong or close to $180^{\circ}$ ). In the following section, we will review the classic stability indicator to appraise the stability of a BWR.

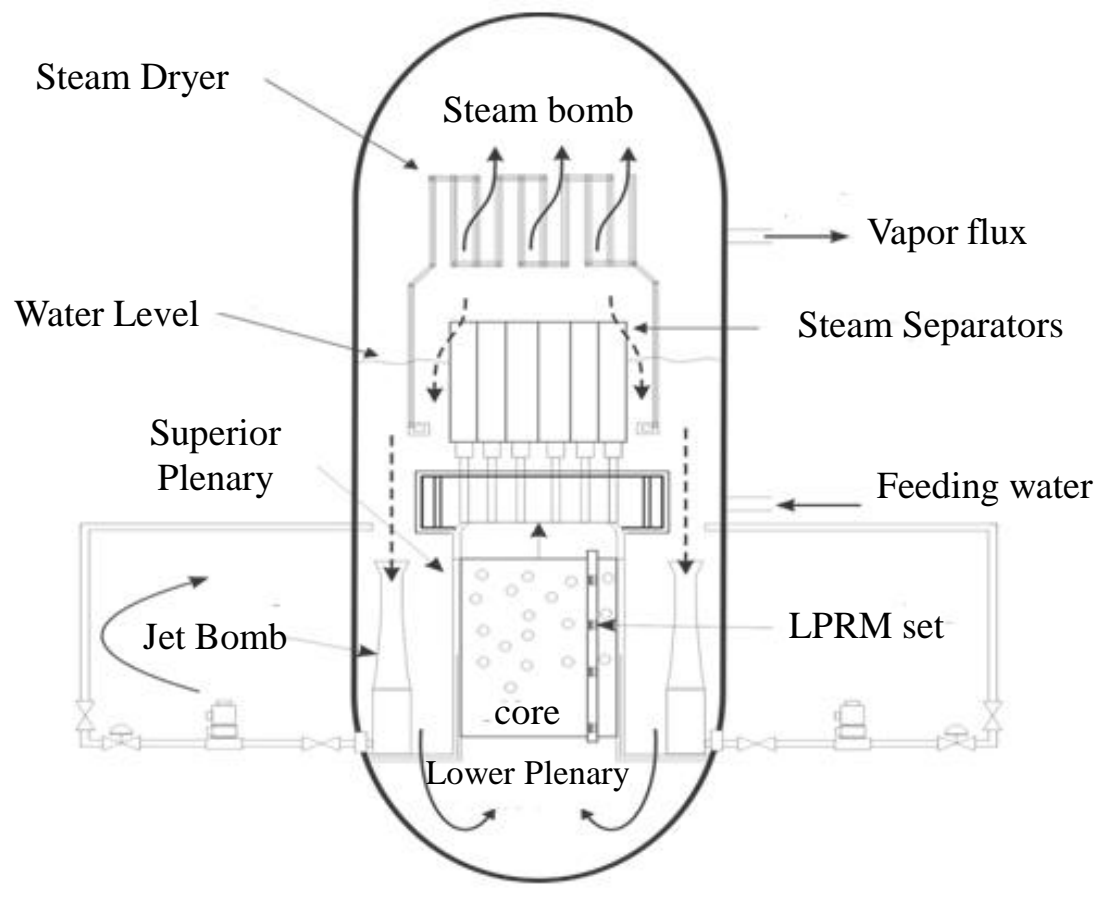

Figure 5. Drawing of a BWR reactor and the longitudinal location of the LPRMs. 


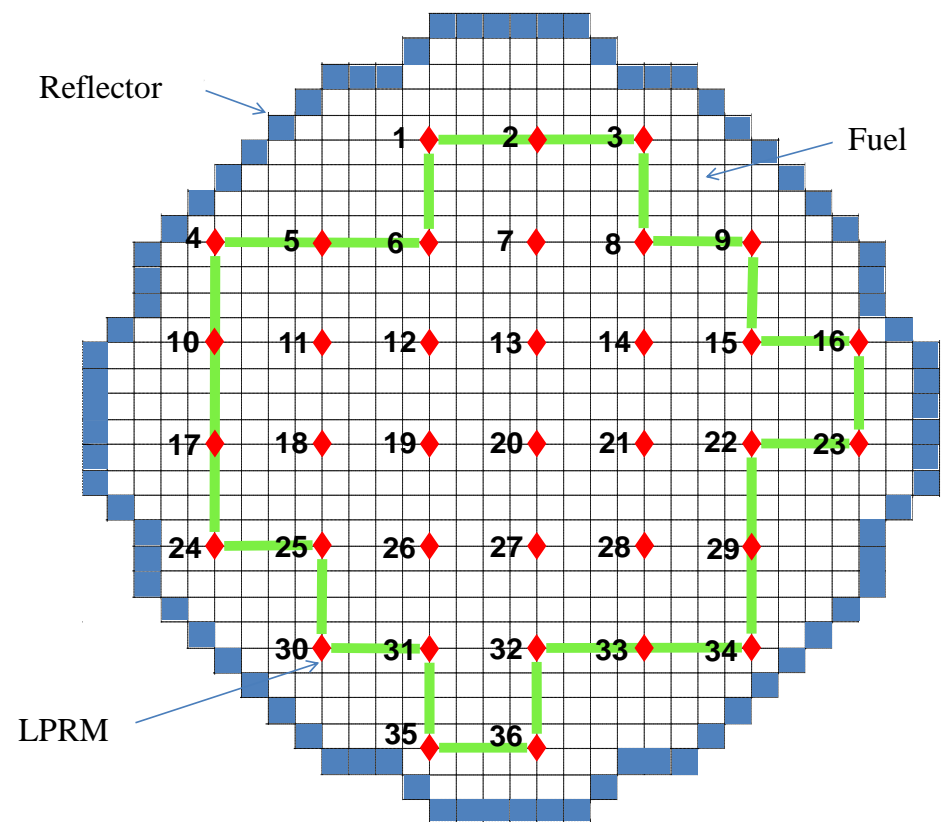

Figure 6. Example of axial placement of LPRMs.

\subsection{Decay Ratio}

Currently, the most common parameter to evaluate BWR stability is known as the decay ratio (DR), which is in most proposals calculated from the impulse response function that stems from an autoregressive (AR) modeling (Williams, 1977; Williams, 1982; Upadhyaya and Kitamura, 1981) of LPRM recordings. The decay ratio is an easy index to scale a margin to the stability boundary and this property is the main output of most stability monitoring systems (Van der Hagen et al., 2000).

\subsubsection{Linear System Dynamics: Stability}

The link between two variables in a system can be best described by a differential equation, if such equation is linear, such equation can be Laplace transformed and the transfer function between two variables can be obtained as the ratio of two polynomials in the s, the Laplace variable (or s domain). The roots of the denominator polynomials are called the poles of the transfer function and the denominator are the zeros. Once the transfer function $H(\mathrm{~s})$ of a linear system is known. The output of this system $Y(\mathrm{~s})$ for any input $X(\mathrm{~s})$ is given by:

$$
Y(\mathrm{~s})=\mathrm{H}(\mathrm{s}) \mathrm{X}(\mathrm{s})
$$


The output $y(\mathrm{t})$ can be obtained in the time domain by applying the a convolution operation between the time domain input $x(t)$ and the impulse response function $h(\mathrm{t})$ (the time domain equivalent of the transfer function):

$$
y(\mathrm{t})=\int_{-\infty}^{+\infty} x(\tau) \mathrm{h}(\mathrm{t}-\tau) d \tau
$$

It is known that $h(\mathrm{t})$ is the response of a system to a delta dirac input function. The features of $h(\mathrm{t})$ determine the stability of the system. If $h(\mathrm{t})$ decays to zero, the output of the system follows the input. However, if $h(\mathrm{t})$ grows in time, old input values are multiplied by a function that increases in magnitude. In this case, the response diverges in time and the system is unstable.

In the Laplace domain, the poles rule the stability of the system. If at least one pole has a positive real part, the impulse response will grow exponentially and the system will be unstable whereas if all the real parts of the poles are negative, the system will be stable. Within the BWR stability domain, we are usually only interested in learning from systems that exhibit oscillatory features when the most positive (and thus unstable) pole is complex. It is in this context that the concepts behind the construction of the DR emerge.

We know, that the impulse response of a system with a pair of complex conjugate poles look like this:

$$
h(\mathrm{t})=\mathrm{e}^{\alpha t} \cos (\omega \mathrm{t}+\varphi)
$$

Where $\alpha$ is the real part and $\omega$ is the imaginary part of the pole. The DR is defined as the ratio between the second and first peaks in the impulse response function (see Figure 7). Which in practice is commonly estimated through AR modeling of LPRM signals. 


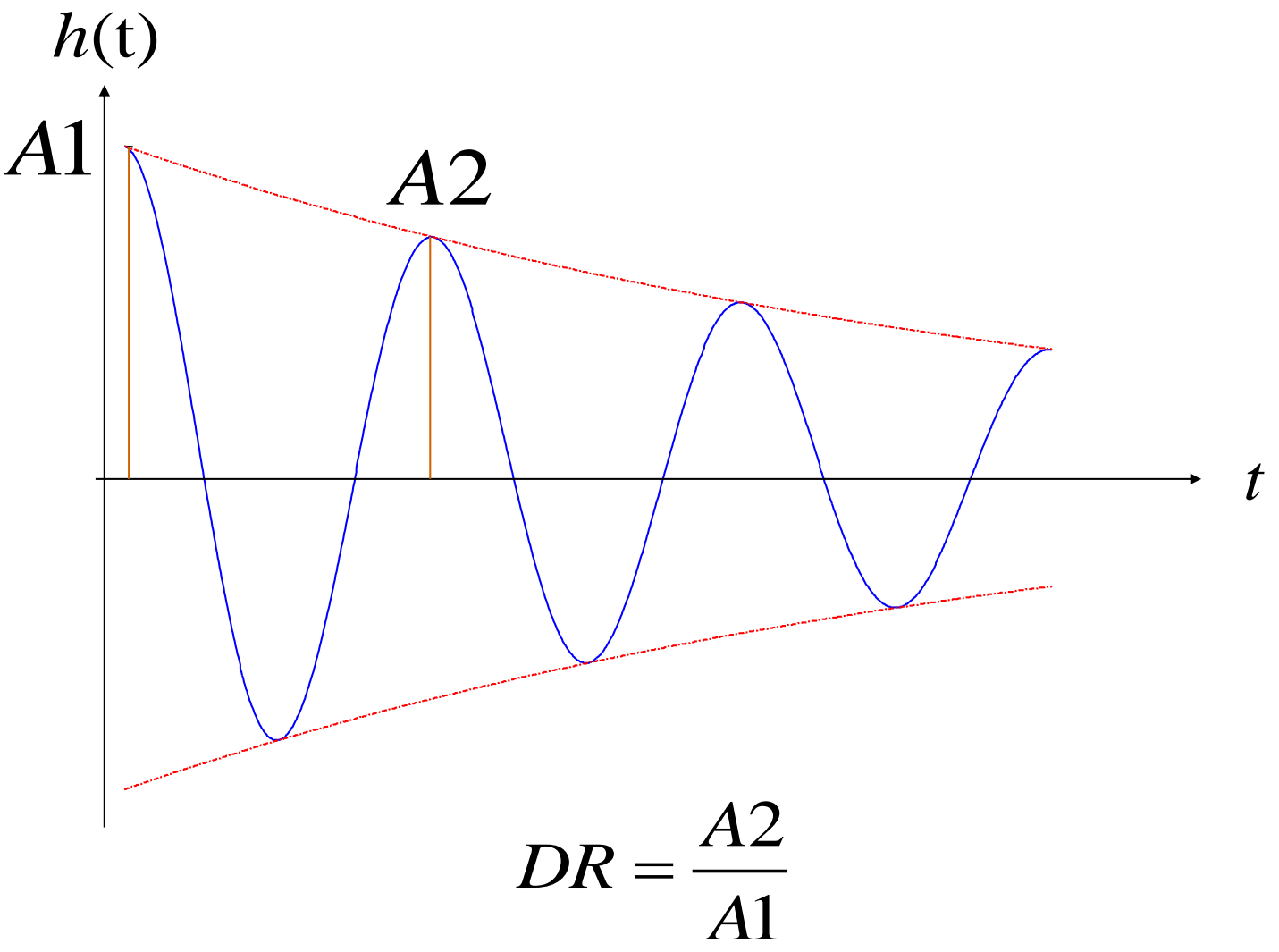

Figure 7. Decay ratio (DR) definition.

This ratio is constant for any two consecutive peaks and equal to (March-Leuba, 1984):

$$
\mathrm{DR}=\exp \left(\frac{2 \pi \alpha}{\omega}\right)
$$

For second order systems, the DR is related to the position of the poles and is a reliable measurement to monitor the stability of a system. The DR is the default indicator to scope the stability of BWRs due to density waves (DWs). For DR validity it is of utter importance to assume that the BWR behaves as a second order linear system with a pair of complex conjugate poles. The latter assumption is unreal in practice, real BWRs are higher order systems with very complex behavior under instability (So, a second order linear modeling of BWRs is not sufficient to assess BWR stability). We therefore consider relevant to study and propose more elaborate stability indicators to assess the stability of a BWR. 


\subsection{Thesis objectives}

The main goal of this work is to develop new BWR stability monitors based on non-linear algorithms to compute indicators (of non-linear nature) that accommodate the complex dynamics of BWR signals recorded by LPRMs.

\subsubsection{Specific Objectives}

1. Study the physical phenomena behind a BWR instability event with the aid of reduced order models (ROMs) that capture the most important features of a BWR.

2. Find the limitations of DR estimates on artificial signals generated by ROMs dedicated to model the dynamics of a BWR.

3. Propose new stability indicators based on non-linear formulas, such as the Shannon Entropy (SE), the Sample Entropy (SampEn) and the Fractal Dimension (FD).

4. Validate the behavior of the proposed novel algorithms and stability indicators with artificial signals generated by ROMs and later by real signals of commercial BWRs.

5. Study the variants of the empirical mode decomposition (EMD, Huang et al., 1998) to decompose real BWR signals.

6. Interpret the results of studying real BWR signals (LPRM data) with the novel indicators.

7. Propose a STABILITY MONITOR with decision rules based on adaptive techniques to analyze LPRM recordings to estimate a non-linear indicator (the best from the 3 indicators mentioned in point 3 ). 


\subsection{Thesis structure}

Chapter 1 is dedicated to introduce the most commonly observed BWR instability type observed in commercial NPPs, which is: the Density wave instability. This chapter also provides a summary of reported BWR instability events and of the instruments inside of the core that are used to appraise the BWR stability for security purposes. Our objectives are also found in this chapter.

Chapter 2 introduces a state of the art of some works that have been published over the past 40 years to analyze BWR instability. Also, the linear or non linear properties of the methods provided in this section are given at the end of this section, along with some of their obstacles for real time implementation.

Chapter 3 Introduces the empirical mode decomposition (EMD), a non linear filter to decompose non-stationary signals that stem from non-linear systems in a multi resolution scheme. This chapter addresses EMD expansions that drew inspiration from the standard EMD to enhance the decomposition of complex data. In this chapter, we also introduce a multivariate expansion of EMD to analyze multi channel data.

Chapter 4 introduces a simple but powerful reduced order model (ROM) of a BWR. The ROM helps to understand the dynamic behavior of a BWR under density wave instability. We perform some experiments with the ROM to test the limitations of the standard DR when it comes to the appraisal of BWR instability. The DR limitations exposed in this chapter open the door to study BWR instability (under density wave) with other more sophisticated tools.

Chapter 5 introduces the first measurement to assess BWR instability (under density wave). The Shannon Entropy (SE), the SE is a non-linear measure of the complexity of a random variable. The Shannon Entropy is used frequently in telecommunications. However, in here we apply it to appraise BWR instability through LPRM recordings. We develop the first non-linear BWR instability methodologies in this chapter with the SE in combination with EMD expansions.

Chapter 6 discusses the second stability measurement (a second proposal) to appraise BWR instability. The new measure is the Sample Entropy (SampEn). The SampEn is a measure that is used for assessing the complexity of physiological time-series signals, diagnosing diseased states. In here it is applied to separate BWR stable states from unstable ones. The SampEn is a sophisticated computer formula to measure the complexity of time series, such formula can be implemented in real time. The SampEn is a much sophisticated indicator that the Shannon Entropy estimator used in chapter 5. We elaborate a BWR stability methodology based on SampEn in combination with a multivariable EMD expansion.

Chapter 7 introduces the third stability proposal (and the most powerful one), the Higuchi Fractal dimension (HFD), a formula that measure the fractal dimension of time series. the fractal dimension is an index of roughness of a time series. The HFD provides reliable 
fractal dimension estimates with few data points (this is useful when there is little data available). The HFD needs only one simple input a priori parameter for operation. We elaborate a BWR stability methodology based on HFD in combination with a multivariable EMD expansion.

Chapter 8 . In here we discuss the constraints of the three studied indicators of the past 3 chapters. The strongest indicator among the three studied ones is chosen to build a robust and practical BWR stability monitor for DW oscillation detection with decision rules. The chosen indicator is the powerful Higuchi Fractal dimension (HFD). 


\section{Chapter 2}

\section{State of the art}

This chapter introduces briefly and classifies some of the most relevant works in BWR stability over the past 40 years. The mentioned works may fall into one of the following major method categories for BWR stability appraisal:

A) Models based on systems of differential equations (also known as reduced order models (ROMs)).

B) Autoregressive (AR) modeling for decay ratio (DR) estimation.

C) Short time Fourier Transform (STFT) and Autocorrelation function (AFC) for DR estimation (Spectral Analysis methods).

D) Bifurcation Analysis.

E) Computer Codes (programs that simulate the dynamic behavior of the reactor, the fine details of such programs are industrial secrets), that provide DR estimates.

F) Wavelet based methodologies for DR estimation.

G) Empirical Mode Decomposition based methodologies for DR estimation.

H) Artificial Neural Networks (ANNs).

\subsection{Reduced order model proposals}

March-Leuba, 1986 developed a reduced order model of the linear dynamic behavior of a BWR. His model is based on studies of various physical processes involved. The proposed ROM is composed of only five differential equations. With the adequate input parameters, the ROM represents accurately the dynamic behavior of BWRs predicted by fine-mesh computations.

Uehiro et al., 1996 proposed an analytical model to study the two-phase flow of in-phase and out-of-phase modes in boiling water reactors. The developed model for parallel boiling channels with the void-reactivity feedback loop, takes into consideration the interaction between channels with different void fraction variations. The authors performed a linear stability analysis of their model in the frequency domain.

Hashimoto et al., 1997 developed a ROM model for linear multichannel analysis of out-ofphase (regional) instability in BWRs. In their model, the zero-power transfer function of a spatial-harmonic mode, the nodal component of the harmonic amplitude and the node-wise feedback coefficients for the mode appear. They apply modal expansion to a transient flux to obtain the transfer function of the model, the nodal components and the feedback coefficients. 
Muñoz-Cobo et al., 2004 developed another reduced order model (ROM) that includes the sub-cooled boiling. They obtained new additional equations for the next magnitudes: the effective inception length, the average void fraction in the sub-cooled boiling region, the average void fraction in the bulk boiling region, the mass fluxes at the boiling boundary and the channel exit. The ROM includes as well a modal kinetics with the fundamental mode and the first sub-critical one, and two channels representing both halves of the reactor core. Their model is able to display out-of-phase oscillations when enough feedback gain is provided.

Lange et al., 2011 developed an advanced reduced order model (ROM). Such model was qualified in the framework of a novel approach for nonlinear stability analysis of BWRs. Their approach is called RAM-ROM method, where RAM is synonym for system code and ROM stands for reduced order model. In the RAM-ROM method, integrated BWR system codes and ROMs are used as complementary tools to examine the stability characteristics of fixed points and periodic solutions of nonlinear differential equations describing the stability behavior of a BWR loop. Their idea is a novel one, because they study the nonlinear processes behind BWR dynamics by applying validated system codes and by applying sophisticated techniques of nonlinear dynamics (e.g. bifurcation analysis).

Dykin et al., 2013 developed another advanced ROM that includes four heated channels and is aimed to study the global and regional BWR instabilities. Their ROM contains three sub-models: a neutron-kinetic model ( that describes neutron transport), a thermal-hydraulic model ( describing fluid transport) and a heat transfer model (that describes heat transfer between the fuel and the coolant). The three models are coupled to each other by two feedback mechanisms: by void feedback and by Doppler feedback. Each sub-model is described by a set of ordinary differential equations, derived from their corresponding timeand space-dependent partial differential equations.

\subsection{Autoregressive modeling for decay ratio estimation}

Mitsutake et al., 1982 applied a multivariable autoregressive (M-AR) model in the simulation study of the core stability test of a BWR. M-AR model technique was used for data analysis of Peach Bottom-2 core stability test, and provided the results of the stability margin of each component and global system in the reactor core. According to the authors their M-AR model is effective for the stability estimation of the reactor core.

Kanemoto et al., 1985 applied an autoregressive (AR) modeling technique in BWRs. They improve the AR model using an associate matrix. In the reactor core, void and recirculation flow noise sources, which are most dominant in BWR plants, are qualitatively evaluated by their improved AR model.

Shi et al., 2001 proposes a reliable method for detecting BWR instability through a nonlinear time series analysis approach namely exponential autoregressive (ExpAR) modeling. The ExpAR model is available for revealing types of nonlinear dynamics such as 
fixed point, limit cycle, and even chaos. Furthermore, the authors claim that the ExpAR is real time estimated to make it suitable for the purpose of on-line BWR instability detection.

Manera et al., 2003 presented a benchmark to compare the performances of exponential autoregressive (ExpAR) models against linear autoregressive (AR) models with respect to BWR stability monitoring. They employ the well known March-Leuba ROM model to generate the time series to be analyzed. They demonstrate that all the methods perform well in determining the stability characteristics of the studied signals.

Some participants that contributed to the Forsmark stability benchmark (Verdú et al., 2003) proposed the AR based methods shown in Table 2.

Table 2. Methods used in the Forsmark stability benchmark.

\begin{tabular}{|c|c|c|}
\hline Method & Organisation & Countries involved \\
\hline $\begin{array}{c}\text { Auto-regressive (AR) } \\
\text { methods and dominant poles }\end{array}$ & PSI, UPV/CSN SIEMENS & $\begin{array}{c}\text { Switzerland, Spain, } \\
\text { Germany/USA }\end{array}$ \\
\hline $\begin{array}{c}\text { Auto-regressive (AR) } \\
\text { methods and impulse } \\
\text { response }\end{array}$ & $\begin{array}{c}\text { TOSHIBA, JAERI, IRI/TU- } \\
\text { Delft, PSU }\end{array}$ & $\begin{array}{c}\text { Japan, The Netherlands, } \\
\text { USA }\end{array}$ \\
\hline $\begin{array}{c}\text { Auto-regressive moving } \\
\text { average (ARMA), plateau } \\
\text { method }\end{array}$ & PSI & Switzerland \\
\hline
\end{tabular}

\subsection{Fourier transform based approaches.}

Yokomizo et al., 1990 developed a stability monitor that calculates the autocorrelation values of two delayed time intervals to compute the damping coefficient, $\alpha$, of the impulse response, $h(t)$, of a BWR core. If the $\alpha$ estimates are positive, the core is stable. However, if they become negative or zero, the core is unstable. The estimated $\alpha$ values can be converted to decay ratios. Due to its simplicity, this method is suitable for online monitoring.

Hotta and Ninokata, 2002 estimated the core stability of the Ringhals unit 1 by numerical random noise that simulates indefinable two-phase flow noise sources in actual cores. Their noise model is expressed as a product of band white amplitude and arbirtrary shape functions. The two authors evaluate BWR instability by means of decay ratios.

Navarro-Esbri et al., 2003 presented several tools to study the time dependence of the linear stability parameters of a BWR using neutron noise analysis. They studied the variation of the fundamental frequency of a signal via the short-time Fourier transform, they make comparisons of such method with calculations of a time dependent Power Spectral Density (PSD) function. The temporal variation of the DR analyzed using a method based on an AR model to fit the different blocks of the signal. 


\subsection{Bifurcation Analysis}

Tsuji et al., 1993 use the bifurcation theory for the stability analysis of BWRs. They derive the stability information by analysis of the bifurcation phenomena on the equilibrium states. Their approach was applied to the analysis of the stability linked with in-phase power oscillations. The loss of linear stability takes place at a lower reactor power as the coolant flow rate decreased, and this instability occurs at the Hopf bifurcation point.

Lange et al., 2013 explored methods developed in the nonlinear dynamics field in order to reveal the nature of BWR stability states when power oscillations are being observed. A powerful method they used is: Bifurcation analysis. They demonstrate via some examples of phenomena which can only be understood in nonlinear terms by application of bifurcation theory and where linear interpretation leads to incorrect conclusions.

\subsection{Wavelet based methodologies for DR estimation}

Espinosa-Paredes et al., 2005 introduced a wavelet-based method to analyze BWR instability events. The proposed methodology includes the following features: a) A shorttime Fourier transform (STFT) analysis, b) decomposition using the continuous wavelet transform (CWT), and c) application of multi resolution analysis (MRA) using discrete wavelet transform (DW). STFT analysis permits the study, in time, of the spectral content of analyzed signals and the originality of the CWT provides information about ruptures, discontinuities, and fractal behavior of studied BWR data.

Espinosa-Paredes et al., 2007 studied BWR unstable power oscillations with another wavelet based method for three different scenarios: a) during stable operation and in b) inphase conditions and c) out-of-phase conditions. The results obtained by the authors suggest that a Wavelet-based method can help with the understanding of the dynamics in BWRs. The stability parameters: oscillation frequency and DR were calculated as a function of time according to the theory of wavelet ridges.

Sunde and Pazsit, 2007 also used the Wavelet transform and Wavelet filtering techniques for the improvement of the estimation of the decay ratio to assess BWR stability. This work is divided into two main areas: the first one is concerned with the improvement of the quality of the auto-correlation function $(\mathrm{ACF})$ for DR estimation. The second area is the estimation of the DR by the use of continuous Wavelet transform (CWT). The CWT appears to be a promising candidate to determine the critical DR even in the case of two oscillations being co-existent with different stability properties. 


\subsection{Empirical mode decomposition based methodologies for DR estimation}

Montesinos et al., 2003 presented for the very first time in BWR stability history an application of the empirical mode decomposition (EMD, Huang et al., 1998) technique to the stability analysis of BWR. Such method decomposes the original time series data in intrinsic oscillation modes or IMFs. Then, for each IMF, the Hilbert amplitude spectrum is computed. From the IMF related to BWR stability, the authors obtained by ordinary autoregressive(AR) modeling the DR value and the oscillation frequency of BWR signals.

Prieto-Guerrero and Espinosa-Paredes, 2014 inspired by the work of Montesinos et al., 2003 decided to develop a method based on the EMD to estimate an indicator associated with instability in BWRs. Such instability indicator is not the classical DR, but it is associated with it. The proposed method allows the decomposition of the studied signal (LPRM or APRM signal) in different levels of intrinsic mode functions (IMFs). One or more of these computed modes can be linked to the instability problem in BWRs. Their alternative $\mathrm{DR}$ is computed by means of the autocorrelation function (ACF) of the IMF linked to the instability event.

Prieto-Guerrero et al. 2015 developed a stability monitor based on the Multivariate empirical mode decomposition (MEMD) (Rehman and Mandic, 2009) to compute DR estimates and to measure out-of-phase oscillations. The MEMD is a method that decomposes multivariate signals into intrinsic mode functions (IMFs) or simply modes that capture the slow and fast oscillations of the targeted signal. The IMFs track the cyclic oscillations related to instability due to DW. The MEMD decomposes non-linear data that stems from non-linear sources. This proposal was an original one because it employed a non-linear technique (the MEMD method) to analyze BWR signals and compute the conventional DR. However, real BWR signals are non-linear and non-stationary and exhibit an intricate behavior that the DR does not accommodate. Thus, it is a must to develop new methodologies and indicators well adapted to accommodate the fine details of BWR signals for use when studying.

\subsection{Computer codes for DR estimation}

March-Leuba and Rey, 1993 made a state of the art of the existing computer codes (programs to simulate the dynamic behavior of BWRs) of their time, such codes can be divided into the following categories:

Frequency domain codes (the most widely used):

- LAPUR

- NUFREQ

- FABLE

Time domain codes (the most widely used): 
- RAMONA-3B

- TRAC-BF1

- TRACG

- RETRAN

- EPA

- SABRE

- TRAB

- TOSDYN-2

- STANDY

- SPDA

LAPUR (Otaduy, 1979; Otaduy and March-Leuba, 1989; March-Leuba, 1990; MarchLeuba and Otaduy, 1983) was developed by the Oak Ridge National Laboratory (ORNL) for the US NRC and is currently used by NRC, ORNL, and others. LAPUR's capabilities include both point kinetics and the first subcritical mode of the neutronics for out-of-phase oscillations. LAPUR's main result is the open and closed-loop reactivity-to-power transfer function from which a DR is estimated.

NUFREQ (Peng et al., 1985) is a family of codes called NUFREQ-N, NUFREQ-NP, and NUFREQ-NPW that compute reactor transfer functions for the fundamental oscillation mode; the main differences between them are the ability to mode pressure as an independent variable (NUFREQ-NP) so that it can reproduce the pressure perturbation tests.

FABLE (Chan, 1989) is a code used by General Electric (GE) which can model 24 radial thermal hydraulic regions that are coupled to point kinetics to estimate reactor transfer function for the fundamental mode of oscillation.

RAMONA-3B (Wulff, 1984; Moberg and Tangen, 1986; Rohatgi, 1990) is a code that was developed jointly by the US NRC and ScandPower; it is used by the Brookhaven National Laboratory (BNL), ScandPower and ABB. RAMONA-3B has a full 3D neutron kinetics model that is capable of coupling to the channel thermal hydraulics in a one-to-one basis. It is costly in CPU time.

TRAC (Weaver, 1986; Andersen, 1983; Shaug, 1990) has two versions currently used in BWR stability analysis. TRAC-BF1 is the open version used by the Idaho National Engineering Laboratory (INEL) and Pennsylvania State University. TRACG is a GEproprietary version. TRAC runs are very expensive in computational time: to minimize this, most runs are limited to the minimum number of thermal hydraulic regions that will do the job. both TRAC versions have full $3 \mathrm{~d}$ neutron kinetics capability.

RETRAN (Jensen and Galer, 1990; McFadden et al., 1981) is a time domain transients code developed by the Electric Power Research Institute (EPRI), it has 1D and point kinetics capability. RETRAN is a relatively fast-running code. It can run on a personal desktop computer. 
EPA(Wulff et al., 1984) stands for Engineering Plant Analyzer. It's a combination of software and hardware that allows real time simulation of BWRs. EPA's software for BWR stability simulations is named HIPA. HIPA uses modeling methods similar to those of RAMONA-3B.

SABRE (Chaiko, 1990) is a time domain code developed and used by the Pennsylvania Power and Light for transient analyses that include BWR instabilities.

TRAB (Valtonen, 1990) is a 1D neutronics code with an average thermal hydraulics region. It was developed and used in the Finish Center for Radiation and Nuclear Safety.

TOSDYN-2 (Takigawa et al, 1987) is a code that was developed by Toshiba Corporation. TOSDYN-2 models multiple channels as well as the balance of plant.

STANDY (Yoshimoto et al., 1990; Muto et al., 1990; Yokomiso et al., 1987) is a time domain code used by Hitachi Ltd. It has 3D neutron kinetics and parallel channel flow across at most 20 thermal hydraulic regions.

SPDA (Haga et al., 1990) is a computer code used by the Japan Institute of Nuclear Safety.

The participants that contributed to the Ringhals stability benchmark (Lefvert, 1996) used the codes shown in Table 3.

Table 3. Methods used in the Ringhals stability benchmark.

\begin{tabular}{|c|c|c|}
\hline Organisation & Code & Frequency/Time Domain \\
\hline CSN/UPV, Spain & LAPUR & Frequency Domain \\
\hline $\begin{array}{c}\text { NETCORP/SCANDPOWER, } \\
\text { USA }\end{array}$ & LAPUR & Frequency Domain \\
\hline NFI, Japan & STAIF-PK, DYNAS-2 & $\begin{array}{c}\text { Frequency Domain, Time } \\
\text { Domain }\end{array}$ \\
\hline PSI, Switzerland & RAMONA-3.5 & Time Domain \\
\hline $\begin{array}{c}\text { SCANDPOWER/ABB } \\
\text { ATOM, Norway/Sweden }\end{array}$ & RAMONA-3 & Time Domain \\
\hline SIEMENS, Germany & STAIF & Frequency Domain \\
\hline TSI, Japan & TSI Stab. Eval. Syst & Frequency Domain \\
\hline $\begin{array}{c}\text { University of Pisa, DCMN, } \\
\text { Italy }\end{array}$ & RELAP5/MOD2 & Time Domain \\
\hline
\end{tabular}

Anegawa et al., 1996 used SIMON $^{\mathrm{TM}}$ on their BWR stability tests. This monitor analyses the core stability margins and plots its status via DRs. The information related to the algorithms used by is classified. This monitor also provides information related to the dephasing of neutron detectors to infer out-of-phase oscillations.

Sometime after the instability event that happened at La Salle in March 1988, the BWR owners group (BWROG) started a project to research endeavors that industries should consider to solve the stability issue as a problem that may appear during operation. As a 
result, the BWROG decided that a long-term solution was required and that such function should have a rapid response that does not rely on the operator. The developed solution is called Long Term solution III (LT-III, Lehmann et al., 1996). LT-III solution is a system that grants reliable, automatic detection and deletion of stability related power oscillations. Option III solution includes three separate methods to detect stability related oscillations: A period based detection method (PBDA, Energy, 1995), a Growth rate algorithm (GRA) and an amplitude based algorithm (ABA). All three techniques perform calculations in OPRM cells (a cell of 1 to 8 LPRMs).

Mowry and Nir, 2001 proposed a stability monitor that is made of a Period Based Algorithm (PBA) and a Confirmation Density Algorithm (CDA). The PBA evaluates the periodicity of the studied LPRMs to detect the presence of density waves (DWs) in the core. The PBA uses a Successive Confirmation Count Model (SCCM), described by Nir and Mowry, 1996. The CDA uses the PBA to identify DWs confirmation, that is, the fraction of LPRMs that reach a successive oscillation period confirmation. When such confirmation density exceeds a fixed stability threshold, a mechanism is triggered to suppress the reactor prior further growth in power of the unstable DW induced oscillation.

Covington and Noël, 2003 proposed a stability monitor that deviated from the norm, i.e. AR modeling to compute the impulse response function of the BWR core, and from the DRs are estimated. Their stability monitor GARDEL-BWR estimates global and regional decay ratios through the explicit use of SIMULATE-3K (S3K). The S3K is the bestestimate coupled neutronic and thermal-hydraulics code, used for performing BWR stability analysis (Grandi et al., 2011). As stated before, in order to trust DR estimates, it is necessary to assume that the BWR signals are stationary and linear.

Grandi et al., 2011 used the coupled neutronic/thermal-hydraulics code, SIMULATE-3K $(\mathrm{S} 3 \mathrm{~K})$, which is a program that many utilities, research institutes and regulatory authorities in Europe use for performing BWR stability analysis of European BWRs. Their work summarizes part of the extensive validation database for the code, and discusses the influence of fuel pin model parameters on the stability analysis.

\subsection{Artificial neural networks}

Tsuji et al., 2005 also developed another stability monitor to compute decay ratios. Their monitor performs a singular value decomposition (SVD) of neutron detector time series into independent extracted components in the sense that their cross-second moments vanish. The SVD procedure is followed by a trained artificial neural network (ANN) to perform an efficient screening of decomposed SVD components to provide reliable DR estimates.

Tambouratzis and Antonopoulos-Domis, 1999 use the smallest possible number of points of the autocorrelation function at the shortest possible time lags as input to a number of back-propagation artificial neural networks (BP ANNs) which are trained to approximate the relationship that exists between the ACF of the neutron noise signals and the DR. Their technique can be implemented in real time. 


\subsection{Time line of techniques proposed to assess BWR stability over the past 40 years}

In this section, we present a time line of the works published over the past 40 years to study the stability of BWRs. Figure 7 shows a time line of many works that have been published since the early 80's to study the phenomena behind BWR instability and how to assess it in commercial BWRs.

1980's: In the 80's, computer codes reigned supreme, in a total of 9 computer codes were developed in this decade. 2 published works proposed in this decade were related to AR techniques and only 1 published work was related to ROMs (March-Leuba, 1986 was a pioneer in the study of BWR instability through ROMs)

1990's: In the early 90's 3 more computer codes were developed and two more appeared in 1996. in the 90s we saw the early development of proposals that studied BWR instability via new methods: such as spectra analysis techniques, artificial neural networks and bifurcation analysis. 2 more ROM proposals appeared in the late 90's to understand the physical aspects behind BWR instability.

2000's: In the time span of 2000-2010, BWR stability methodologies based on digital signal processing techniques became more dominant as computer power grew. Spectral analysis techniques dominated this decade along with proposals based on AR modeling. in 2003, the EMD was used for the first time within the BWR stability community. 
STANDY, 1987
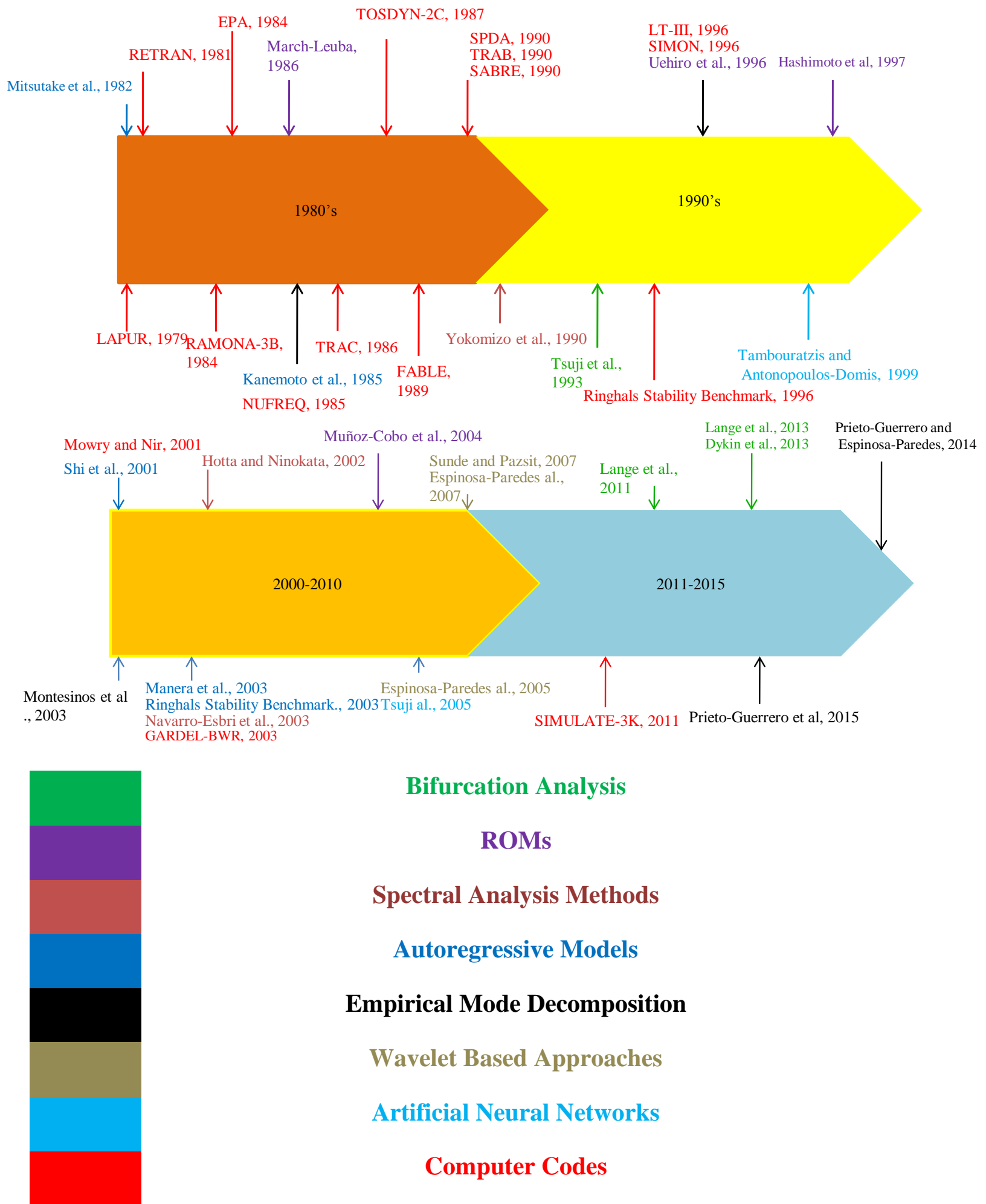

Figure 7. Time line of BWR stability analyses methodologies over the past 40 years. 
2011-2015: in the short time span of 2011 to 2015, non-linear techniques started to become more popular to address the BWR instability problem. A total of 3 bifurcation analysis methodologies were published from 2011 to 2013, only one new computer code appeared in 2011. And in 2014 and 2015 new BWR stability methodologies based on the EMD were published (the work of Montesinos et al., 2003 was the source of inspiration for these two proposals). These two final works published in 2014-2015 (based on the empirical mode decomposition) are the source of inspiration for the work presented in this thesis. EMD expansions (multivariate versions and improved versions of the default EMD) are also used in this work to develop new BWR stability appraisal methodologies. The EMD is an empirical algorithm and to this day, there is no sufficient mathematical theory to sustain it. But, nevertheless, its results in real signal analysis problems are impressive (Huang et al., 1998).

Finally, Table 4 distinguishes the linear proposals from the non linear ones.

Table 4. Linear/Non linear features of BWR instability methodologies given in this section.

\begin{tabular}{|c|c|c|}
\hline Categories & Authors & $\begin{array}{c}\text { Linear techniques / Non- } \\
\text { linear techniques }\end{array}$ \\
\hline Reduced Order Models (ROMs) & $\begin{array}{c}\text { March-Leuba, 1986 } \\
\text { Uehiro et al., 1996 } \\
\text { Hashimoto et al., 1997 } \\
\text { Muñoz-Cobo et al., } \\
\text { 2004 } \\
\text { Dykin et al., 2013 }\end{array}$ & $\begin{array}{c}\text { Linear techniques, DR is } \\
\text { computed via transfer } \\
\text { function of proposed } \\
\text { ROM. }\end{array}$ \\
\hline \\
\hline
\end{tabular}




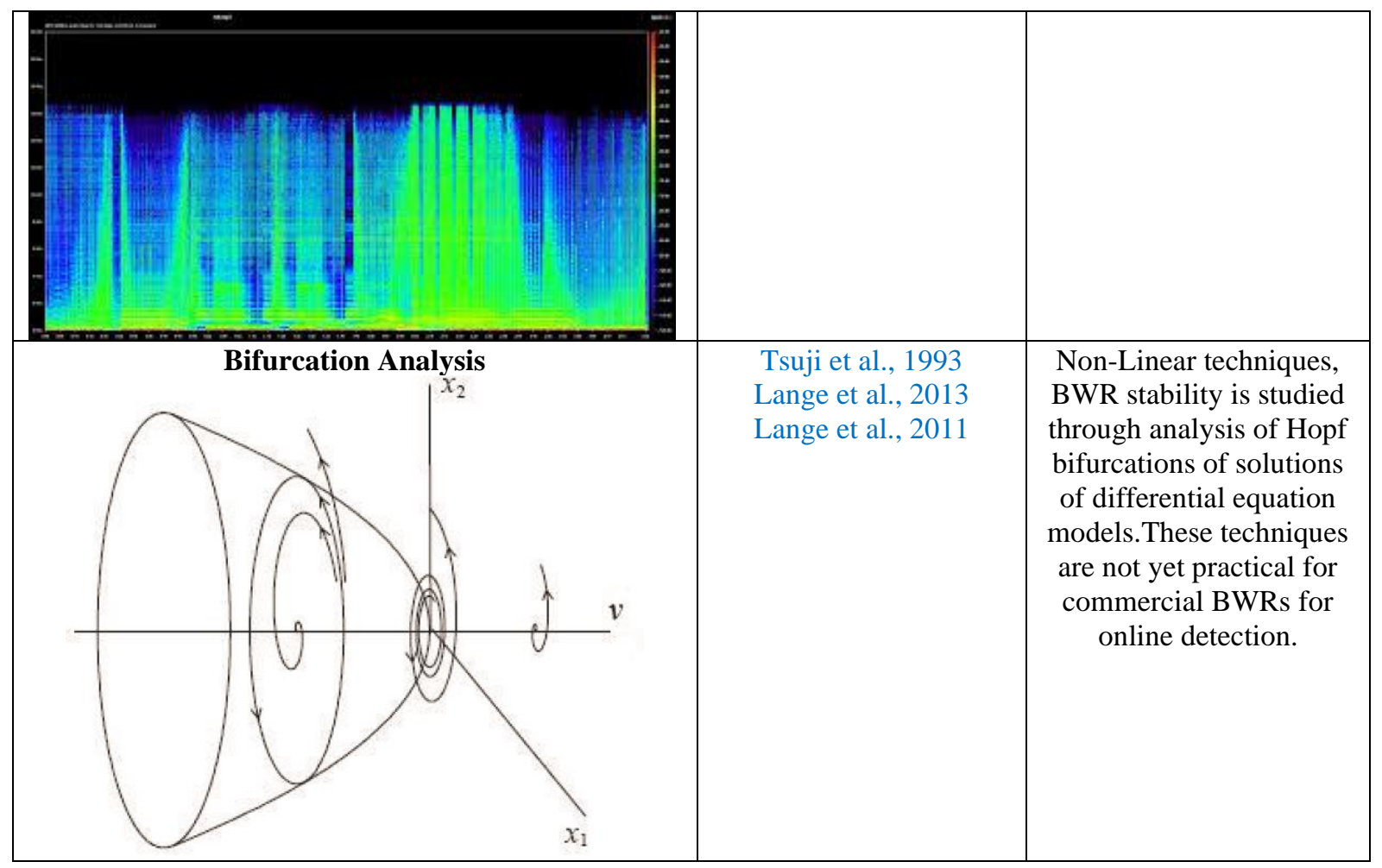

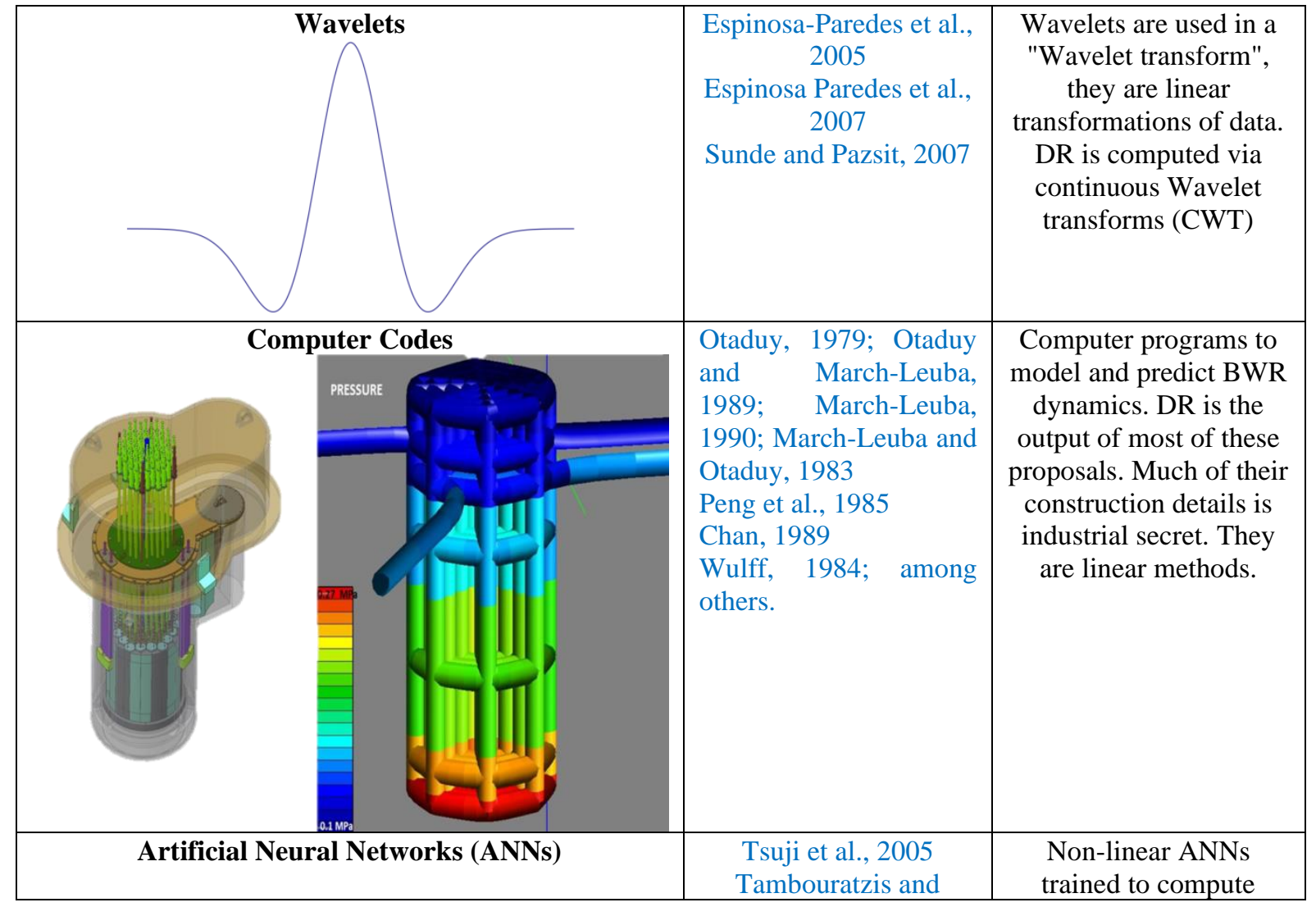




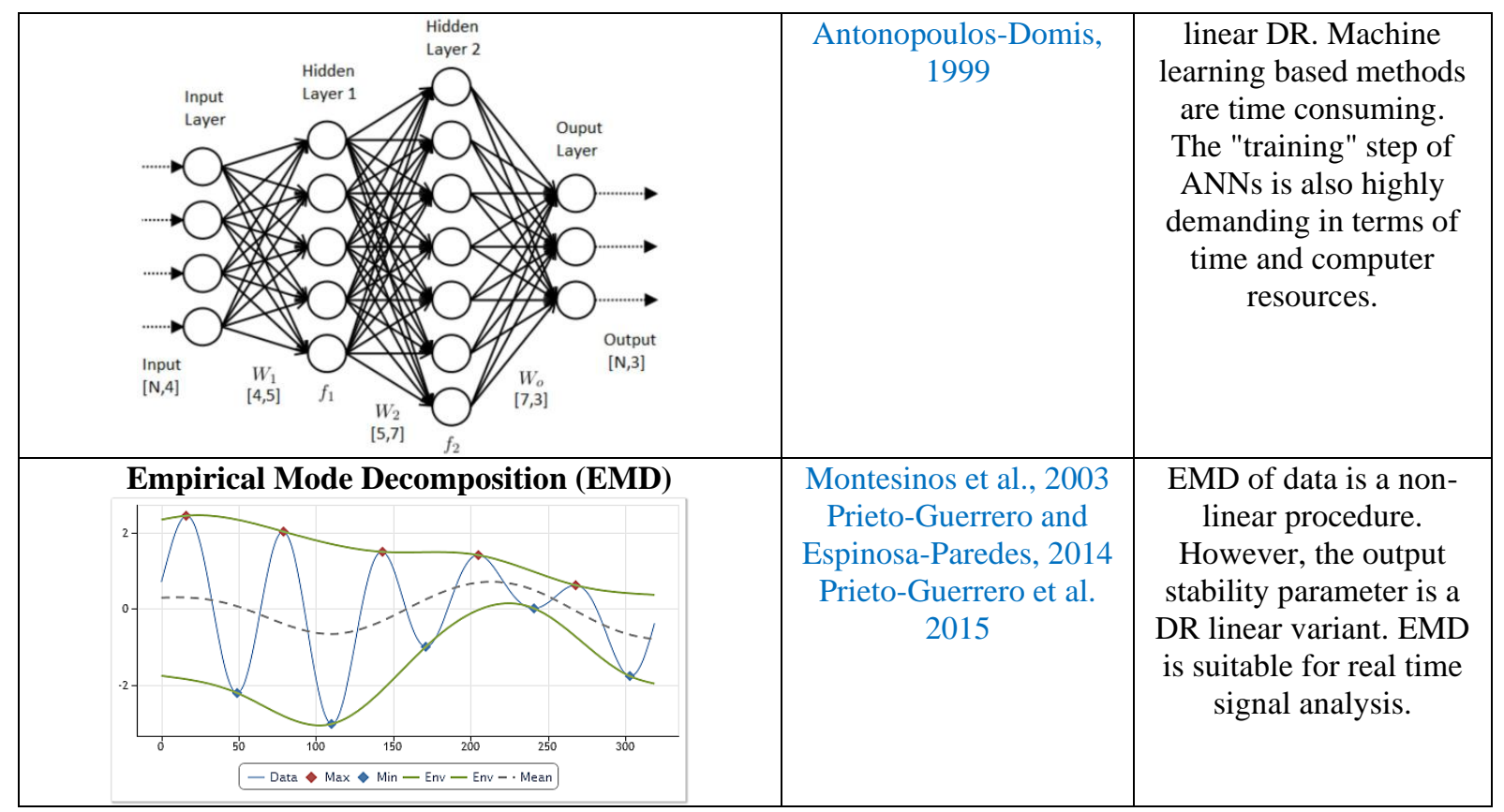




\section{Chapter 3}

\section{The Empirical Mode Decomposition (and its expansions)}

This chapter introduces in detail the default empirical mode decomposition (Huang et al., 1998), an adaptive non-linear filter that is applied to study LPRMs to assess the stability of the BWR plant. Also, in this chapter, we introduce the some EMD expansions that address some issues of the default EMD when it comes to the analysis of intermittencies and transitory behavior of studied data. This thesis is a direct continuation of the work of PrietoGuerrero and Espinosa-Paredes, 2014 and of Prieto-Guerrero et al., 2015 that started with the contributions of Montesinos et al., 2003 to the BWR stability discipline.

\subsection{The default empirical mode decomposition}

The default EMD grants the decomposition of non-stationary signals that stem from nonlinear sources, into various intrinsic mode functions (IMFs) or simply modes. The IMFs capture the fast and slow oscillations that compose the studied signal. The original signal can be reconstructed if all the extracted IMFs are added together. The default EMD has been applied to:

1. Study Seismic signals (Han and Van der Baan, 2013; Wang et al., 2012; Yu and Zhang, 2017; Ivan, 1999).

2. In the analysis of Electrocardiogram(ECG) signals (Kumar et al., 2018; Tan et al., 2014; Weng et al., 2006; Yang et al., 2015).

3. In the analysis of Climate Variability signals (Coughlin and Tung 2014; Molla et al., 2011; Molla et al., 2006).

The IMFs must satisfy the next properties:

1. The number of extrema (maxima and minima) and the number of zero-crossings must be equal or differ at most by one.

2. The local mean, defined as the mean of the upper and lower envelopes, must be zero.

Method 1 (default EMD): The default EMD method can be described by the next steps, but first, let $x$ be the signal of interest to decompose into IMFs:

Step 1. Set $k=0$ and find all extrema of $r_{o}=x$.

Step 2. Interpolate between minima (maxima) of $r_{k}$ to obtain the lower (upper) envelope $\mathrm{e}_{\min }\left(\mathrm{e}_{\max }\right)$. 
Step 3. Compute the mean envelope $m=\left(\mathrm{e}_{\min }+\mathrm{e}_{\max }\right) / 2$.

Step 4. Compute the IMF candidate $d_{k+1}=r_{k}-m$.

Step 5. Is $d_{k+1}$ an IMF?

- Yes, save $d_{k+1}$, compute the residue $r_{k+1}=x-\sum_{i=1}^{k} d_{i}$, do $k=k+1$, and treat $r_{k}$ as input data in step 2.

- No, treat $d_{k+1}$ as input data in step 2.

Step 6. Continue until the final residue $r_{k}$ satisfies some predefined stopping criterion.

The refinement process (steps 2 to step 5) needed to extract every IMF, requires a certain number of iterations named as siftings. The extracted modes $d_{k}, k=1,2, \ldots, K$ decompose $x$ and are in theory, nearly orthogonal to each other. To, illustrate how the EMD works, consider the next artificial signal $x$ :

$$
x=x(t)=\cos (2 \pi 2 t)+\cos (2 \pi 1 t)+\cos (2 \pi 0.5 t)
$$

The sampling frequency of $x$ is $20 \mathrm{~Hz}$. Figure 8 shows a $10 \mathrm{~s}$ plot of this signal. Figure 9 shows the EMD decomposition of $x$. A total of 4 IMFs were extracted from the studied signal, the last one (in red) is the residue of the decomposition. The EMD decomposes any studied signal in IMFs. Oscillations are extracted from highest frequency components to lowest frequency components. The first IMF (IMF 1) has captured the oscillation associated to $\cos (2 \pi 2 \mathrm{t})$, the second one (IMF 2$)$ has captured the oscillation linked to $\cos (2 \pi 1 \mathrm{t})$, the third IMF (IMF 3 ) captured the lowest frequency oscillation of $x$ : $\cos (2 \pi 0.5 \mathrm{t})$. The last IMF (IMF 4$)$ is the residue of the decomposition. The decomposition is adaptive and data driven. However, the EMD is highly dependent on the sampling frequency of studied signals. We do not know how many IMFs will be extracted from a signal (the EMD lacks control of the number of IMFs to be computed). There is no mathematical theory to explain how the EMD works. Therefore, we must rely heavily on numerical simulations to extract as much information as possible from the EMD algorithm. As Huang et al., 1998 wrote in their proposal: We do not need to understand in detail how the digestive system works in order to eat. The EMD has shown impressive results in other disciplines of science (as described above). So, we have decided to continue using the EMD to analyze LPRM signals to assess BWR instability. We know by experience that the EMD is a trustworthy method to decompose non-stationary signals that are the output of nonlinear dynamical systems (there is no such thing as linearity in nature). 


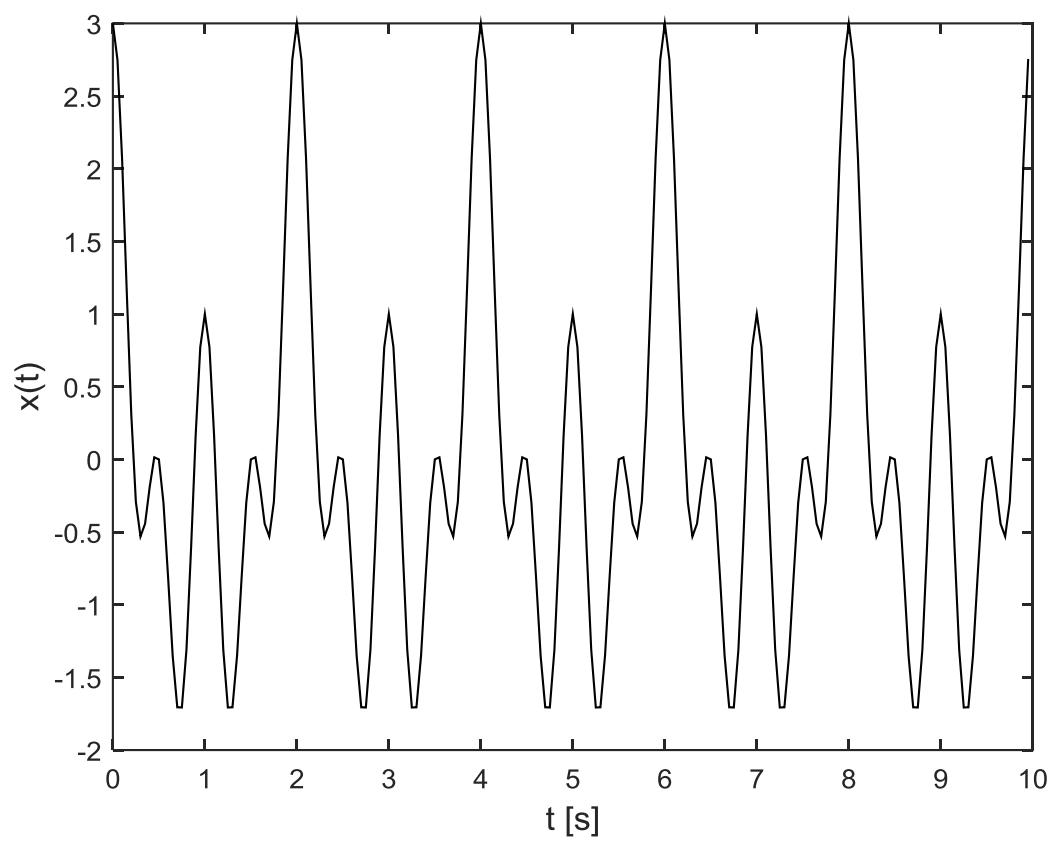

Figure 8. Artificial signal $x(t)$.
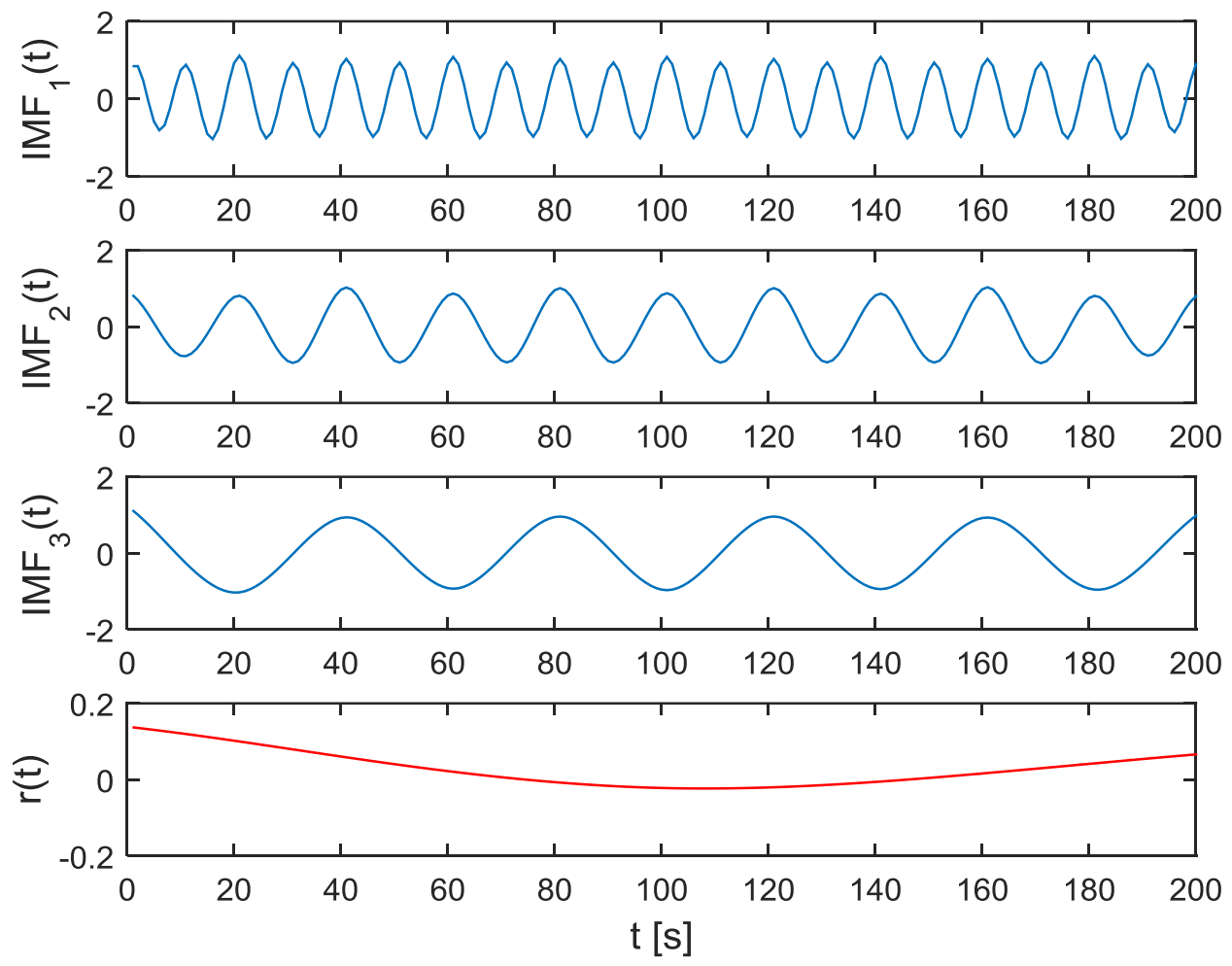

Figure 9. EMD of artificial signal $x(t)$. 
Figure 10 shows the EMD decomposition of a real BWR signal, an LPRM recording of 15 s that belongs to LPRM 3 of case 4 of the Forsmark instability benchmark (Verdú et al., 2003). This segment was decomposed through EMD, the results of the decomposition are shown in Figure 11. When it comes to BWR signals, we are interested in isolating the IMF associated to BWR instability (Montesinos et al., 2003), such IMF looks like a clean cyclic oscillating function of frequency close to $0.5 \mathrm{~Hz}$. The EMD decomposed the segment into 5 IMFs (see Figure 11). The first IMF is probably associated to noise of the acquisition equipment system and to noise produced by the recirculation pump. The second IMF looks like an oscillating function (although not fully clean) and it might be linked to BWR density wave phenomena, although a frequency analysis is required to confirm this (which will come later in this document). IMFs 3 to 5 are low frequency components and are related mainly to the reactor control system. The EMD decomposed the targeted signal segment in a multi resolution fashion and in an adaptive way and data driven way (no need to pre select a mother Wavelet, as is the case for Wavelet based transformations). As we have mentioned before, we have no control of the number of IMFs that will be extracted, in this case, the EMD decomposed the segment into 5 IMFs, if such IMFs are added together, we will recover the original signal. The EMD accommodates non-stationary and non-linear behavior of the studied signal and as such the IMFs are physically meaningful. There are however a few issues that might appear in the default EMD results, which we will discuss in the next subsection.

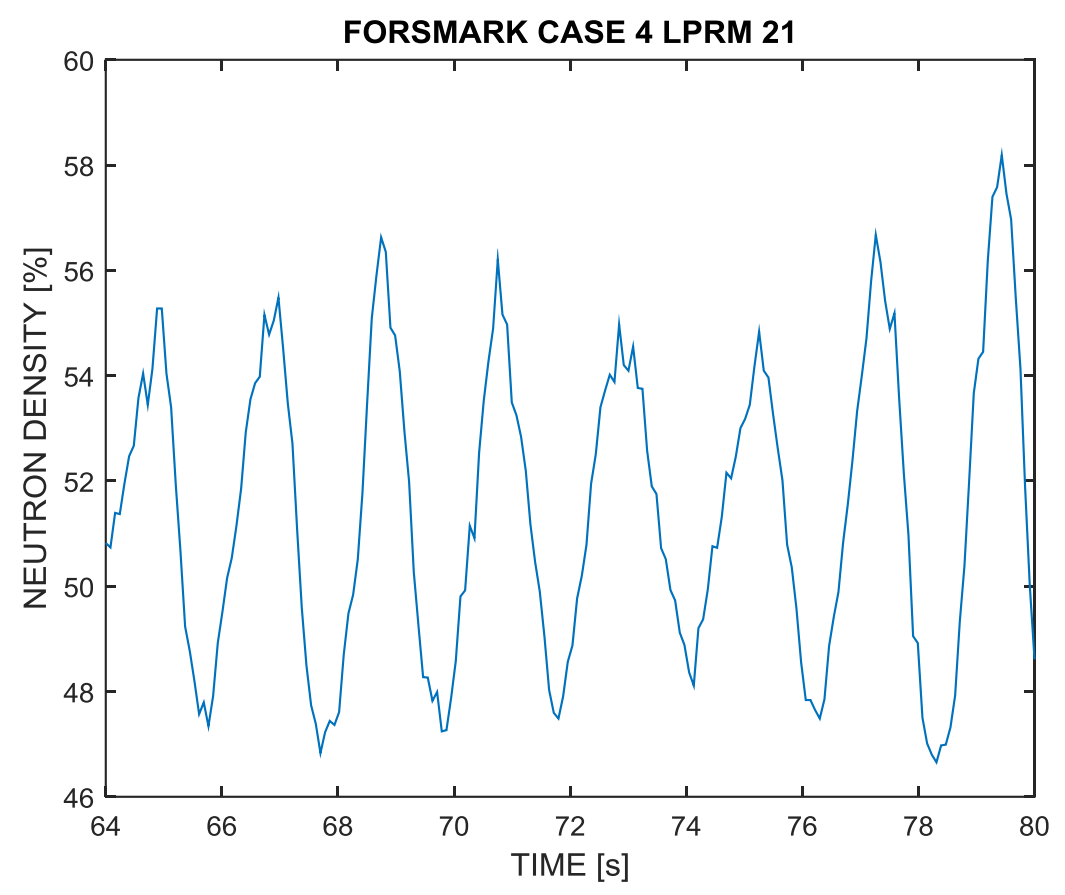

Figure 10. Real BWR signal: LPRM recording of $15 \mathrm{~s}$. 


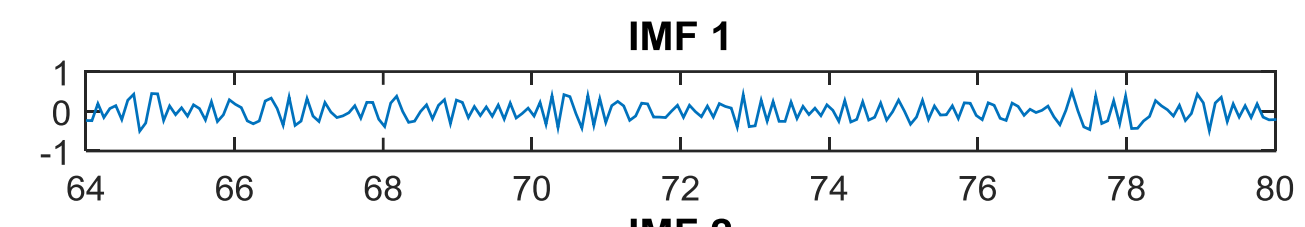

IMF 2
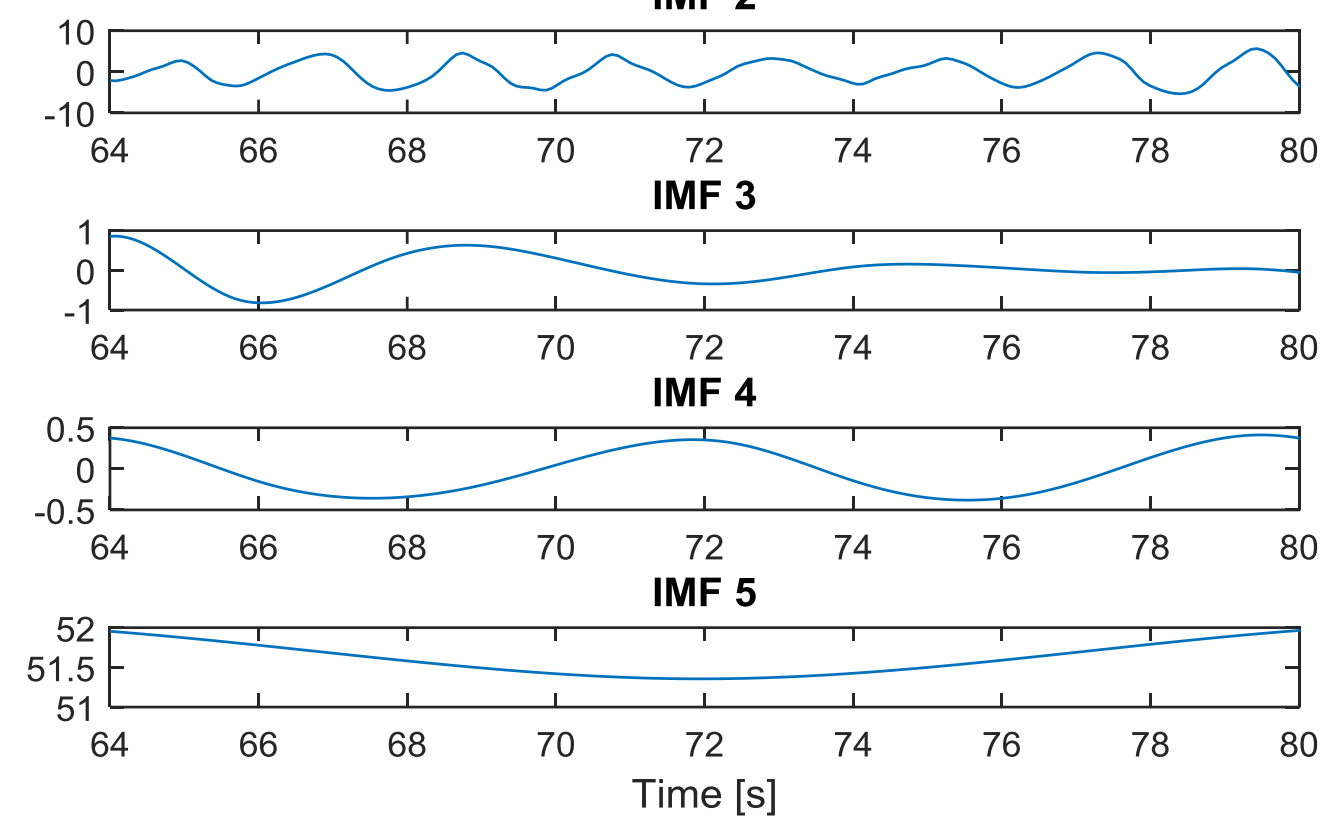

Figure 11. EMD of a real BWR signal.

\subsubsection{The mode mixing problem}

One of the major disadvantages of the EMD is the frequent appearance of an issue that is known as mode mixing, which is defined as a single intrinsic mode function (IMF) either consisting of signals of widely disparate scales, or a signal of a similar scale residing in different IMF components. This issue might spoil the meaning of individual IMFs. To alleviate this drawback, an interesting property of the EMD must be exploited: such property appears when the signal to decompose is a white Gaussian noise. When this Gaussian noise is decomposed, the EMD behaves as an adaptive dyadic filter bank, as the one shown in Figure 12. Where 5000 independent time series (of white Gaussian noise) of 512 points each have been generated, and the average spectra of the first seven IMFs are plotted as a function of the normalized frequency. The methods that are discussed in the following, are expansions of the default EMD to address the mode mixing issue. The idea behind such methods is to add an ensemble of realizations of white noise to the signal of interest. The goal of such non-intuitive action is to repair and exploit the dyadic filter bank property of the EMD to improve the IMF separation of the targeted signal. 


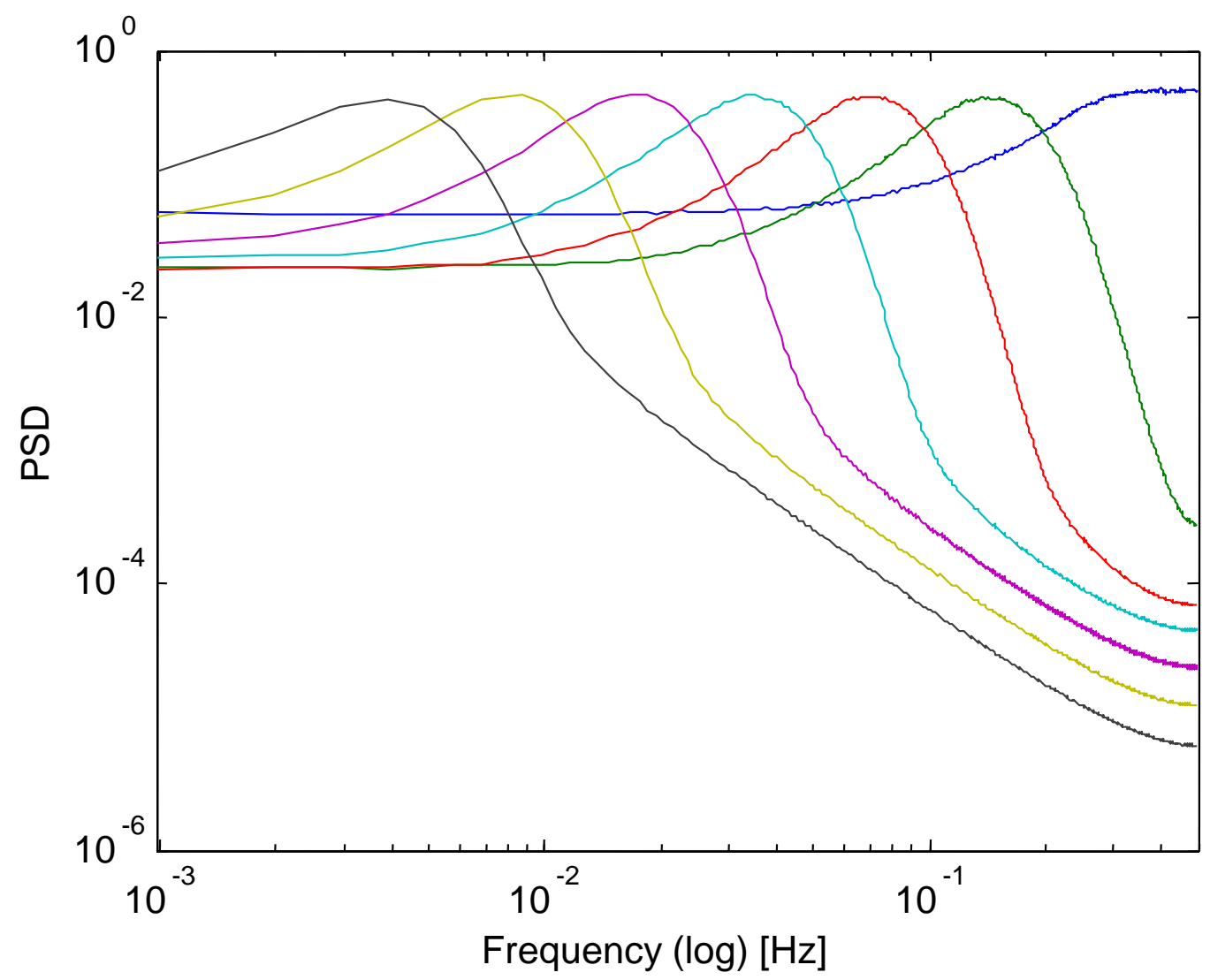

Figure 12. EMD equivalent filter bank for a white Gaussian noise.

\subsection{The ensemble empirical mode decomposition}

In order mitigate the mode mixing problem, the ensemble empirical mode decomposition (EEMD) method was introduced by Wu and Huang, 2009. In the EEMD method, the true modes are the average of the corresponding IMFs obtained from an ensemble $I$ of the original signal plus different realizations of white noise of standard deviation $\beta$. This approach is based on the insight gleaned from recent studies on the statistical properties of white noise, which showed that the EMD is effectively an adaptive dyadic filter bank when applied to white noise. These authors proved that noise could help data analysis in the EMD, through the exploitation of the filter bank property of the EMD, illustrated in Figure 12. The EEMD has been applied before in:

Rotor fault diagnosis of rotating machinery (Lei et al., 2009), in improving forecasting accuracy (Wang et al., 2015). In the fault diagnosis of locomotive roller bearings (Lei et al., 2011) and in BWR instability (Montalvo et al., 2017) for the very first time in BWR instability history. 
Method 2 (The ensemble empirical mode decomposition) The EEMD is described as follows:

1. Generate $x^{(i)}=x+\beta w^{(i)}$ where $i=1,2, \ldots, I . w^{(i)}$ is a zero mean unit variance white noise realization of standard deviation $\beta$ and $I$ is the number of noise realizations.

2. Decompose completely each $x^{(\mathrm{i})}$ by the default EMD, obtaining the modes $\mathrm{IMF}_{j}^{(i)}$. Where $j=1, \ldots, N$ indicates the corresponding mode.

3. Assign $\overline{\mathrm{IMF}}_{j}$ as the $j$-th mode of $x$, obtained by averaging the corresponding modes: $\overline{\mathrm{IMF}}_{j}=\frac{1}{I} \sum_{i=1}^{I} \mathrm{IMF}_{j}^{(i)}$.

The extraction of every $\mathrm{IMF}_{j}^{(i)}$ requires a different number of sifting iterations. It can be noticed that in the EEMD, every $x^{(i)}$ is decomposed independently from the other realizations and for every one of them a residue $r_{j}^{(i)}=r_{j-1}^{(i)}-\mathrm{IMF}_{j}^{(i)}$ is obtained at each stage, with no connection between the different realizations, such is the cause of some EEMD disadvantages:

- The decomposition is not complete.

- Different realizations of signal plus noise might produce different number of modes.

Figure 13 shows the EEMD decomposition of the previous LPRM recording of $15 \mathrm{~s}$ that belongs to LPRM 3 of case 4 of the Forsmark instability benchmark. A total of 7 IMFs were extracted by the EEMD, The first 2 IMFs are associated to acquisition noise and to the noise produce by the recirculation pump. IMF 3 looks like a clean cyclic oscillation, our experience might indicate that this IMF is related to BWR density wave instability (OlveraGuerrero et al., 2017), the oscillation looks clean (We are interested in recovering a oscillating function of a frequency close to $0.5 \mathrm{~Hz}$ and IMF 3 looks visually like that). IMFs 4 and 5 are low frequency oscillations probably related to reactor control system and are irrelevant for DW instability appraisal. As time passed, more authors started to develop better EMD upgrades to address the mode mixing issue, EEMD was the first proposal in that family of expansions. In the following subsections we will review two more modern methods to address the mode mixing phenomena. In the work of Prieto-Guerrero and Espinosa-Paredes, 2014, the authors built their proposal with the default EMD. They did not take into account the existence of the mode mixing problem. Our goal now is to upgrade the methodology they proposed by replacing the default EMD for the most reliable EMD expansion that addresses the mode mixing issue existing in default EMD. One issue of the EMD expansions is the computer time they require to do the decomposition of the studied signal. 


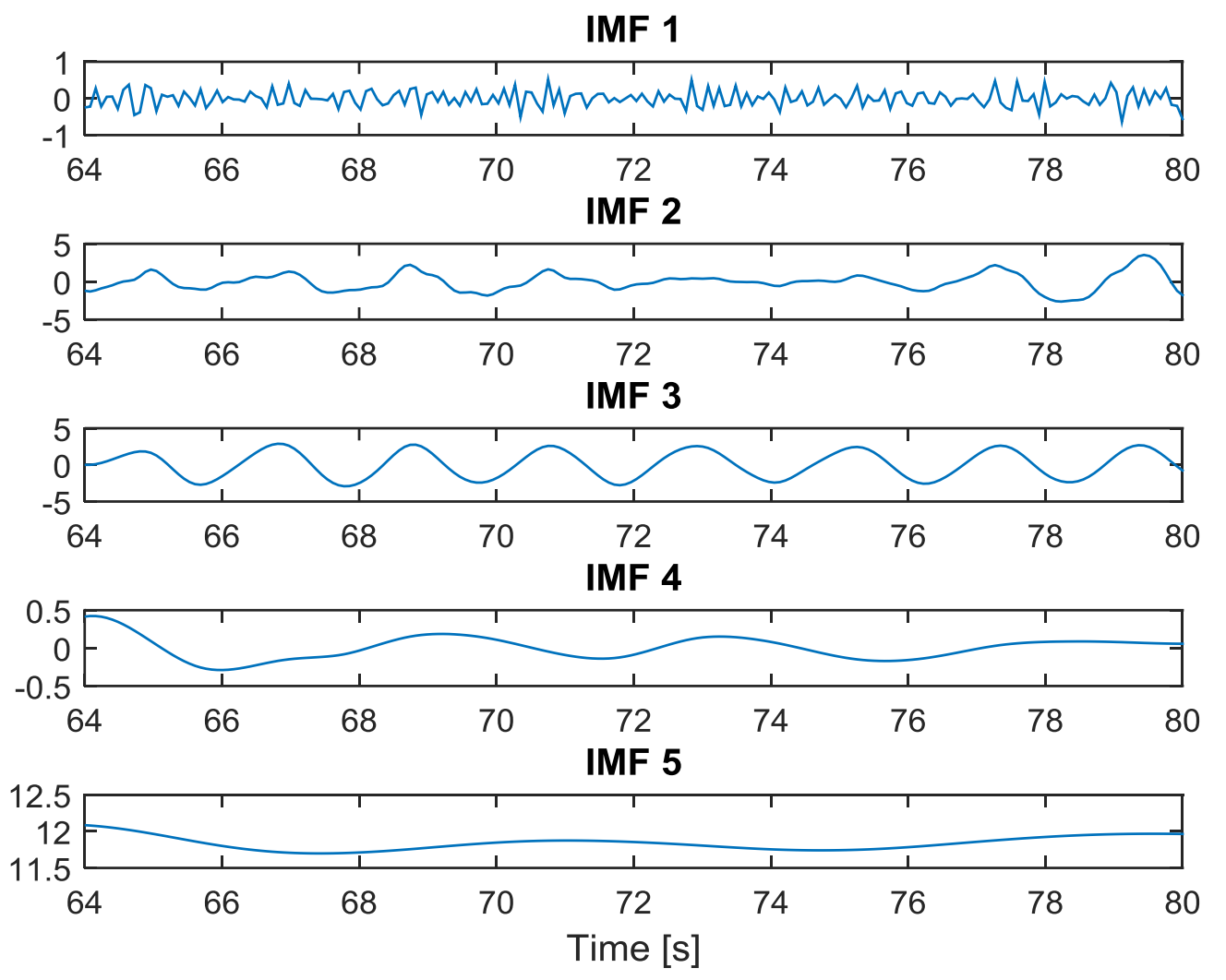

Figure 13. EEMD of a real BWR signal ( $I=100$ and $\beta=0.1$ ).

\subsection{The complete ensemble empirical mode decomposition with assisted noise}

The word "complete" presumably refers to decomposing completely everything, even added perturbations such as noise. The EEMD relies on averaging the modes obtained by EMD by applying several realizations of additive Gaussian white noise to the original signal. The resulting decomposition solves the mode mixing problem. However, it introduces new ones. In the method here discussed, a particular noise is added at each stage of the decomposition and a unique residue is computed to obtain each mode. The resulting decomposition is complete, with a numerical negligible error. Such EMD expansion is called the complete ensemble empirical mode decomposition with assisted noise (CEEMDAN) and was proposed by Torres et al., 2011 and has been applied widely in the next disciplines:

Wind speed forecasting (Zhang et al., 2017). In ECG signal denoising (Xu et al., 2017) and in fault diagnosis of rolling element bearings (Lei et al., 2017). 
Method 3 (The CEEMDAN) The complete ensemble empirical mode decomposition with assisted noise is described as follows (Torres et al., 2011):

Observe that in the EEMD, each $x^{i}$ is decomposed independently from the other realizations and so for each one a residue $r_{k}^{i}=r_{k-1}^{i}-\mathrm{IMF}_{k}^{i}$ is obtained.

In the CEEMDAN, the decomposition modes, the decomposition modes will be noted as $I M F_{k}$. The CEEMDAN authors proposed to calculate a unique first residue as:

$$
r_{1}=x-I M F_{1}
$$

where $I M F_{1}$ is obtained in the same way as in EEMD. Then, compute the first EMD mode over an ensemble of $r_{1}$ plus different realizations of a given noise obtaining IMF 2 by averaging. The next residue is defines as: $r_{2}=r_{1}-I M F_{2}$. This procedure continues with the rest of the modes until the stopping criterion is reached. Let us define the operator $E_{j}(\square)$ which, given a signal, produces the $j$-th mode obtained by EMD. Let $w^{i}$ be white noise with $N(0,1)$. If $x$ is the targeted data, we can describe our method by the following algorithm:

1. Decompose by EMD realizations $x+\varepsilon_{o} w^{i}$ to obtain their first modes and compute:

$$
I M F_{1}=\frac{1}{I} \sum_{i-1}^{I} I M F_{1}^{i}=\overline{I M F}_{1} .
$$

2. At the first stage $(k=1)$ calculate the first residue as in equation (6): $r_{1}=x-I M F_{1}$.

3. Decompose realizations $r_{1}+\varepsilon_{1} E_{1}\left(\mathrm{w}^{i}\right), \mathrm{i}=1, \ldots, I$, until their first EMD mode and define the second mode: $I M F_{2}=\frac{1}{I} \sum_{i-1}^{I} E_{1}\left(\mathrm{r}_{1}+\varepsilon_{1} \mathrm{E}_{1}\left(\mathrm{w}^{i}\right)\right)$.

4. For $k=2, \ldots, K$ calculate the $k-$ th residue: $r_{k}=r_{(\mathrm{k}-1)}-I M F_{k}$.

5. Decompose realizations $r_{k}+\varepsilon_{k} E_{k}\left(\mathrm{w}^{i}\right)$, with $i=1, \ldots, I$ until their first EMD mode and define the $(\mathrm{k}+1)-$ th $\operatorname{mode}$ as: $I M F_{(\mathrm{k}+1)}=\frac{1}{I} \sum_{i-1}^{I} E_{1}\left(\mathrm{r}_{k}+\varepsilon_{k} \mathrm{E}_{k}\left(\mathrm{w}^{i}\right)\right)$.

6. Go to step 4 for next $k$.

Steps 4 to 6 are performed until the obtained residue is no longer feasible to be decomposed ( the residue does not have at least two extrema). The final residue satisfies: $R=x-\sum_{k-1}^{K} I M F_{k}$ 
With $K$ the total number of modes. Thus, the given signal $x$ can be expressed as:

$$
x=\sum_{k-1}^{K} I M F_{k}+R
$$

Equation (7) makes the proposed decomposition complete and provides an exact reconstruction of the original data. The coefficients $\varepsilon_{i}$ allow to select the SNR (signal to noise ratio) at each stage. Concerning the amplitude of the added noise, Wu and Huang, 2009 use small amplitude values for data dominated by high-frequency signals and vice versa. In this work, we used a few hundred realizations and fixed SNR for all the stages ( $\varepsilon_{j}=\varepsilon_{o}$ in all the stages). Figure 14 shows the CEEMDAN decomposition of the previous LPRM recording of $15 \mathrm{~s}$ that belongs to LPRM 3 of case 4 of the Forsmark instability benchmark. The IMF that is probably associated to BWR instability is IMF 4, this IMF visually looks like an oscillating function (whose frequency according to our feeling must be close to $0.5 \mathrm{~Hz}$ ). IMFs $1-3$ are probably linked to noise and IMF 5 is probably related to reactor control system. This CEEMDAN method seems to work well, although more complex to implement in practice. This technique was later improved, in the following subsection we will review the features of this improved version.

IMF 1
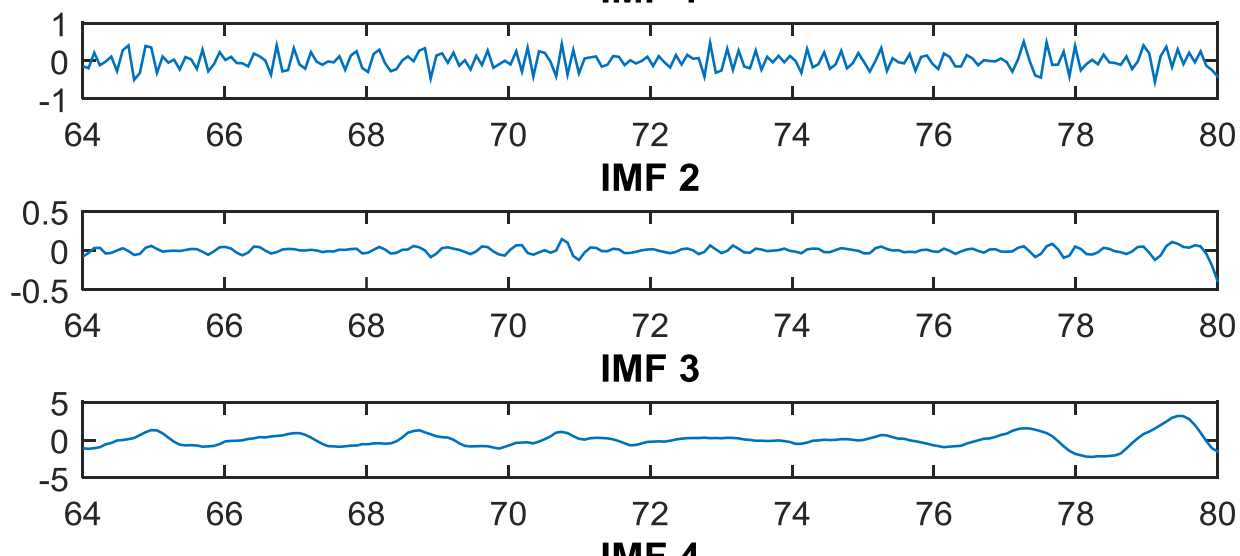

IMF 4

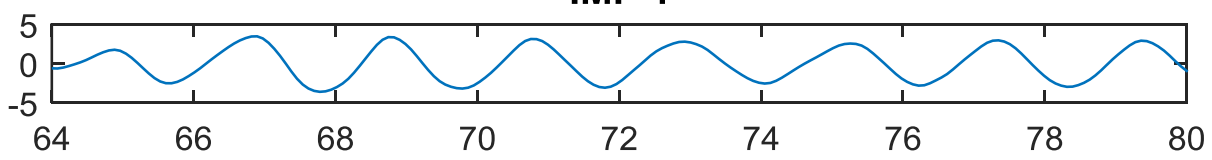

IMF 5

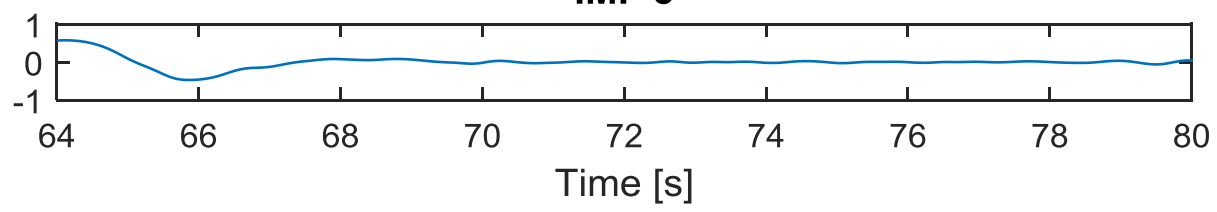

Figure 14. CEEMDAN of a real BWR signal ( $I=100$ and $\varepsilon_{o}=0.1$ ). 


\subsection{The improved complete ensemble empirical mode decomposition with assisted noise}

Taking into account the EEMD drawbacks, the CEEMDAN method was proposed, its authors proved how CEEMDAN overcomes the main difficulties of EEMD, but the method still has two problems. These issues, the presence of residual noise in the modes and the existence of spurious modes, are addressed by a recent improvement of the CEEMDAN technique, proposed by Colominas et al., 2014. Let $w^{(i)}$ be a realization of white Gaussian noise with zero mean and unit variance. Let us define $M(\square)$ as the operator which produces the local mean( the average of the upper and lower envelopes of the studied signal interpolated by cubic splines) of the signal it is applied to and let $\langle\square\rangle$ be the action of averaging throughout the realizations. The improved CEEMDAN has been applied in: The analysis of body signals for glucose detection ( $\mathrm{Li}$ and $\mathrm{Li}, 2016$ ), in Health degradation monitoring (Lv et al., 2018) and in speech signal analysis for mental disorder diagnostic (Alimuradov et al., 2019).

Now, With the previously defined operators $E_{j}(\square), M(\square)$ and $\langle\square$ in mind, the improved CEEMDAN is given by the next steps:

Method 4 Improved CEEMDAN algorithm:

1. Calculate by EMD the local means of I realizations $x^{(i)}=x+\beta_{o} E_{1}\left(\mathrm{w}^{(i)}\right)$ to obtain the first residue: $r_{1}=\left\langle M\left(\mathrm{x}^{(i)}\right)\right\rangle$.

2. At the first stage $(j=1)$ calculate the first mode: $I M F_{1}=x-r_{1}$.

3. Estimate the second residue as the average of local means of the realizations

$$
\begin{aligned}
& r_{1}+\beta_{1} E_{2}\left(\mathrm{w}^{(i)}\right) \quad \text { and } \quad \text { define the second mode: } \\
& I M F_{2}=r_{1}-r_{2}=r_{1}-\left\langle M\left(\mathrm{r}_{1}+\beta_{1} \mathrm{E}_{2}\left(\mathrm{w}^{(i)}\right)\right)\right\rangle .
\end{aligned}
$$

4. For $j=3, \ldots, \mathrm{N}$ calculate the $j-$ th residue: $r_{j}=\left\langle M\left(\mathrm{r}_{j-1}+\beta_{j-1} \mathrm{E}\left(\mathrm{w}^{(i)}\right)\right)\right\rangle$.

5. Compute the $j$-th mode: $I M F_{j}=r_{j-1}-r_{j}$.

6. Go to step 4 for the next $j$.

Constants $\beta_{j}=\varepsilon_{j} \operatorname{std}\left(r_{j}\right)$ are chosen to obtain the desired SNR between the added noise and the residue to which the noise is added, nonetheless, in this work, we fixed the same SNR for all the stages of this procedure $\left(\varepsilon_{j}=\varepsilon_{o}\right)$. Studies about this parameter can be found in Colominas et al., 2012. Figure 15 shows the improved CEEMDAN decomposition of the previous LPRM recording of $15 \mathrm{~s}$ that belongs to LPRM 3 of case 4 of the Forsmark instability benchmark. IMF 3 looks like a cyclic oscillation (probably linked with the DW 
instability phenomena), IMFs 1-2 are linked to the acquisition noise and IMF 4 and 5 are low frequency components not associated with the Density wave phenomena (no useful information can be extracted from them). Only a total of 5 IMFs are shown in this figure out of a total of 7 IMFs extracted by this method. The improved CEEMDAN was selected to build many of the BWR instability proposals described in this work (read chapter 4) at the univariate level.
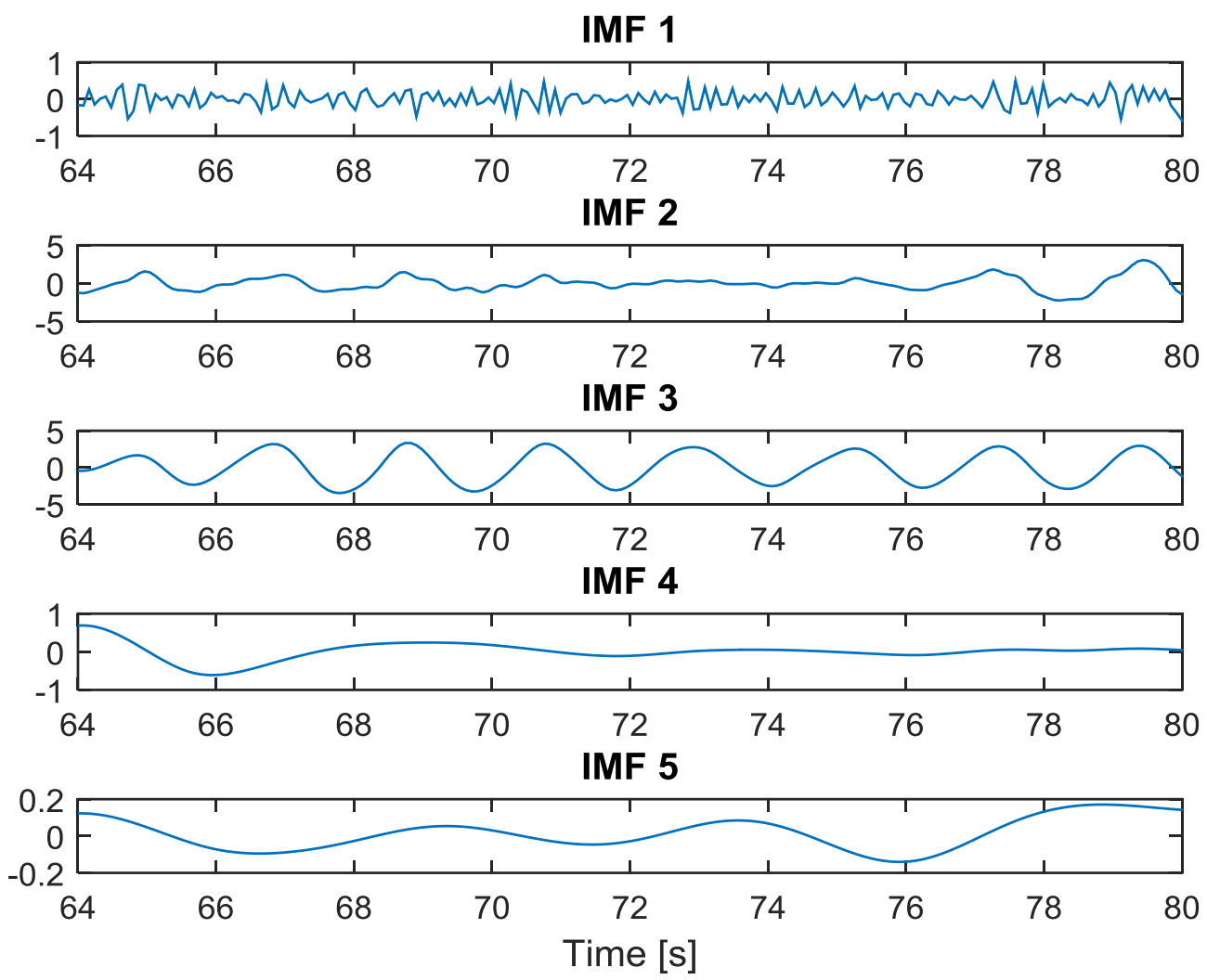

Figure 15. Improved CEEMDAN of a real BWR signal ( $I=100$ and $\varepsilon_{o}=0.1$ ).

\subsection{The Hilbert-Huang transform}

Consider the next analytic representation of a signal $x$ :

$$
Z_{n}=x+j \hat{x}=A e^{j \varphi}
$$

Where $A$ and $\varphi$ are the envelope and the instantaneous phase of the signal, respectively. In this equation $\hat{x}$ is the Hilbert transform (Benedetto, 1996) of the signal $x$, and is calculated as: 


$$
\hat{x}=x * 1 / \pi n
$$

A convolution product between $x$ and the infinite impulse response filter $1 / \pi n$. The Hilbert-Transform can be thought of as a phase shift of $x$ by $\pi / 2$ radians. As a result $x$ and $\hat{x}$ are said to be quadrature. This complex or analytic signal is completely characterized by its amplitude $A$ and its phase $\varphi$ with values in the interval $[0,2 \pi)$, forming a canonical pair. Consider the following:

$$
x=\operatorname{Re}\left[\mathrm{Z}_{x}\right]=\operatorname{Acos}(\varphi)
$$

It is observed that this canonical representation of $x$ corresponds to a signal varying in amplitude and phase all the time. Based on this representation, to estimate the instantaneous frequency linked to the instantaneous phase $\varphi$. From equation $Z_{x}=A e^{j \varphi}$. The instantaneous frequency is defined as:

$$
\omega_{\text {inst }}=d \varphi / d n
$$

The analytic signal $Z_{x}$ associated with $x$ has the same amplitude and frequency range with $x$. It also comprises the phase information of the original signal $x$. According to this fact, we can construct the analytic signal corresponding to each IMF using the HilbertTransform. The combination of EMD (or of any EMD expansion) applied to the signal $x$ to generate IMFs, and the Hilbert transform of each IMF is called the Hilbert-Huang transform (HHT). Since HHT is not based on the Fourier transform, the time-frequency resolution is not limited by uncertainty and there is no need to assume that the studied signals (LPRM signals) are stationary or linear.

\subsubsection{HHT on an artificial signal}

Consider the signal given in equation (5): $x=x(t)=\cos (2 \pi 2 t)+\cos (2 \pi 1 t)+\cos (2 \pi 0.5 t)$ illustrated again in Figure 16. Figure 17 illustrates again its EMD decomposition, into 4 IMFs. However, only the first 3 IMFs are shown in this figure because the fourth IMF is the residue of the decomposition. The HHT of each IMF is also illustrated in red. The HHT grants the instantaneous frequency (IF), which is a time - frequency representation of an IMF function. the first IF oscillates around $2 \mathrm{~Hz}$, the second IF oscillates strongly around 1 $\mathrm{Hz}$ and the last one (IF 3) is an oscillation close to $0.5 \mathrm{~Hz}$. The IFs are not rooted in the Fourier transform. Thus, this frequency-time representation is not limited by the constraints of the Fourier transform and it might serve as an alternative to study the frequency content of a time series. We highlight that there are still some difficulties with the HHT procedure on the edges of a signal (near the start and near the end of the time series). In the following subsection the HHT is tested with IMFs extracted from a segment of a real BWR signal (recorded by LRPMs). The studied BWR signal stems from case 4 of the Forsmark stability benchmark. 


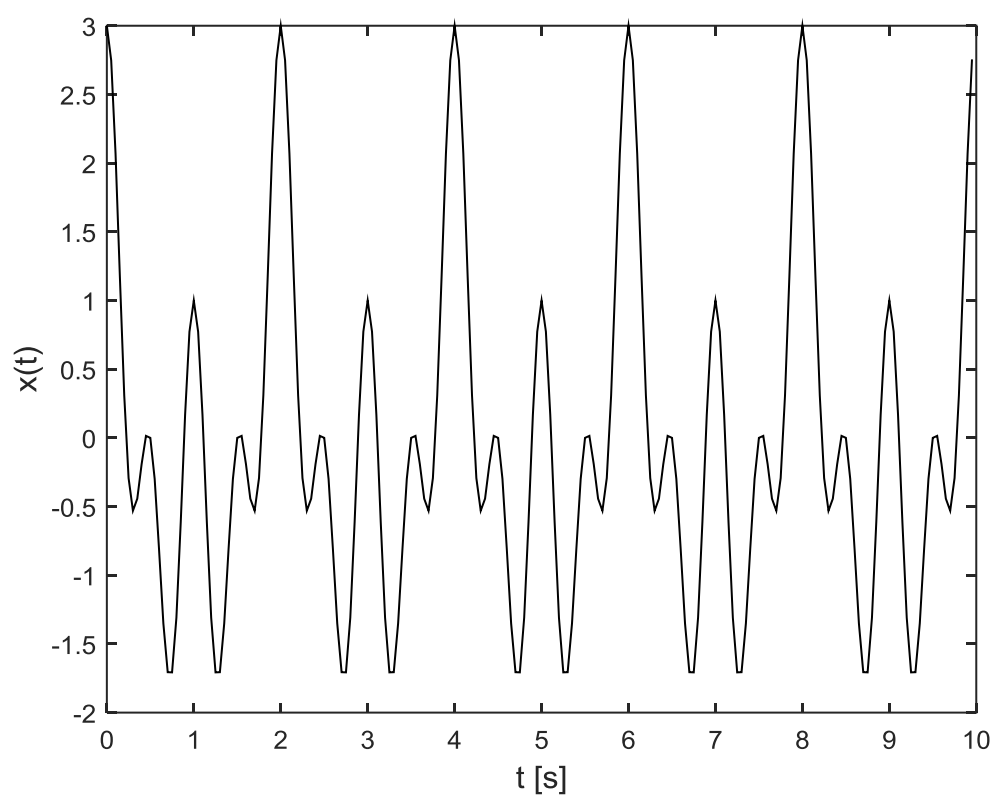

Figure 16. Artificial signal: $x=x(\mathrm{t})=\cos (2 \pi 2 \mathrm{t})+\cos (2 \pi 1 \mathrm{t})+\cos (2 \pi 0.5 \mathrm{t})$.
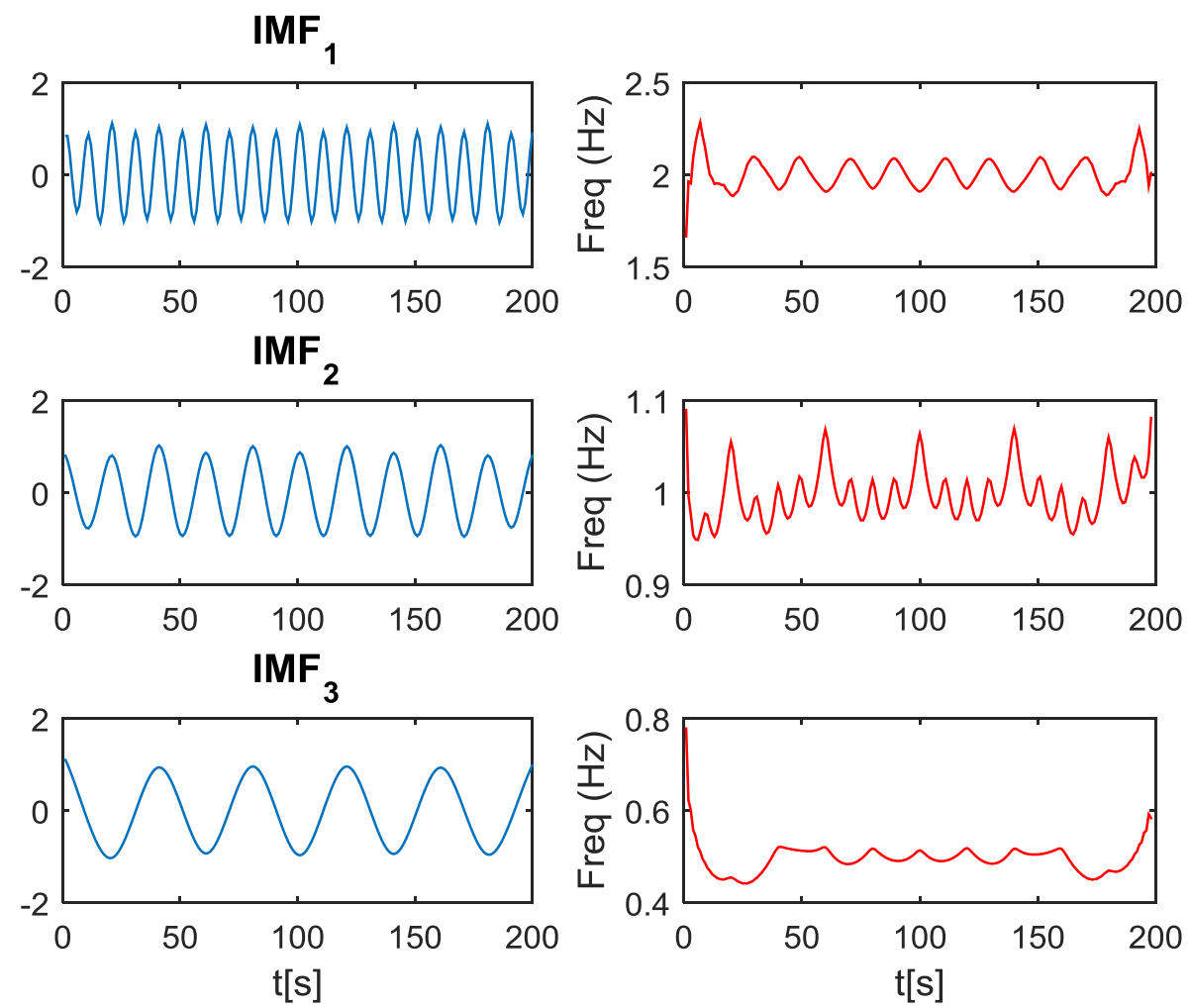

Figure 17. EMD decomposition of the Artificial signal along their IFs (estimated by means of HHT). 


\subsubsection{HHT on a real LPRM signal}

Figure 18 shows the previous EMD decomposition of a real BWR signal, an LPRM recording of $15 \mathrm{~s}$ that belongs to LPRM 3 of case 4 of the Forsmark instability benchmark (Verdú et al., 2003). However, now, the instantaneous frequency (IF) granted by the HHT is also shown (in red). The IF of IMF 1 is a high frequency oscillating around $5 \mathrm{~Hz}$. The IF of IMF 2 oscillates close to $0.5 \mathrm{~Hz}$ (IMF 2 is the IMF linked to the density wave instability). The IF of IMF 3 oscillates close to $0 \mathrm{~Hz}$ and the IF of IMF 4 oscillates between $0.1 \mathrm{~Hz}$ and $0.15 \mathrm{~Hz}$. We highlight that IMF 2 looks visually like a cyclic function with an IF close to $0.5 \mathrm{~Hz}$, this IMF 2 is probably linked to DW instability whereas the other 3 computed IMFs are unrelated to the DW problem.
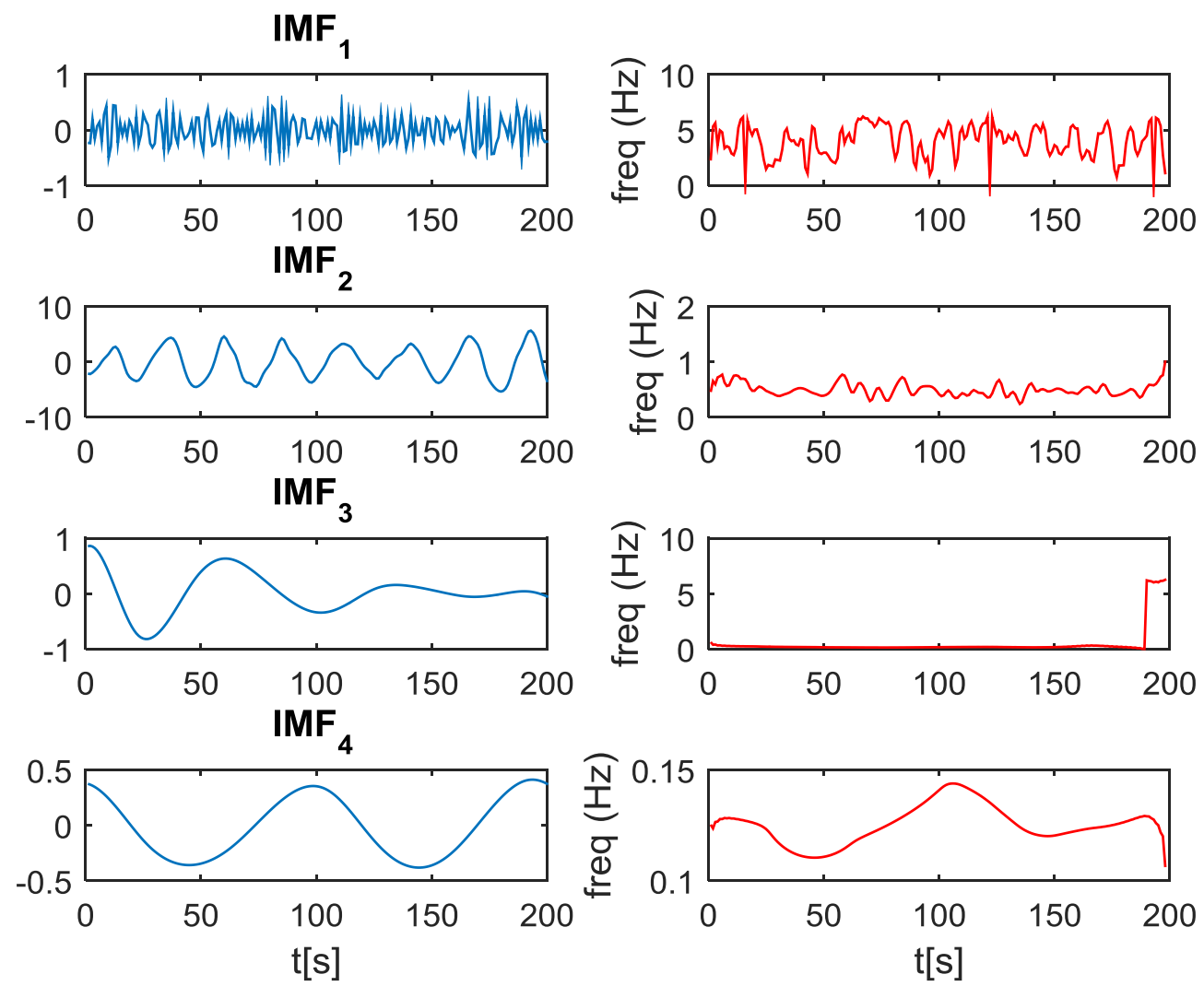

Figure 18. EMD of a real BWR signal along the IFs of each IMF.

Figure 19 shows the same LPRM 3 signal segment decomposed by EEMD to address the mode mixing issue of the default EMD. But now, the IF of each IMF is shown too. The IF of IMF 1 oscillates around $5 \mathrm{~Hz}$. The IF of IMF 2 oscillates close to $0.5 \mathrm{~Hz}$. IF of IMF 3 oscillates close to $0.1 \mathrm{~Hz}$ and IF of IMF 4 changes from 0 to $5 \mathrm{~Hz}$ in a quasi-squared wave fashion. The IMF linked to BWR instability is IMF 2 (its associated IF oscillates around 0.5 $\mathrm{Hz})$. 

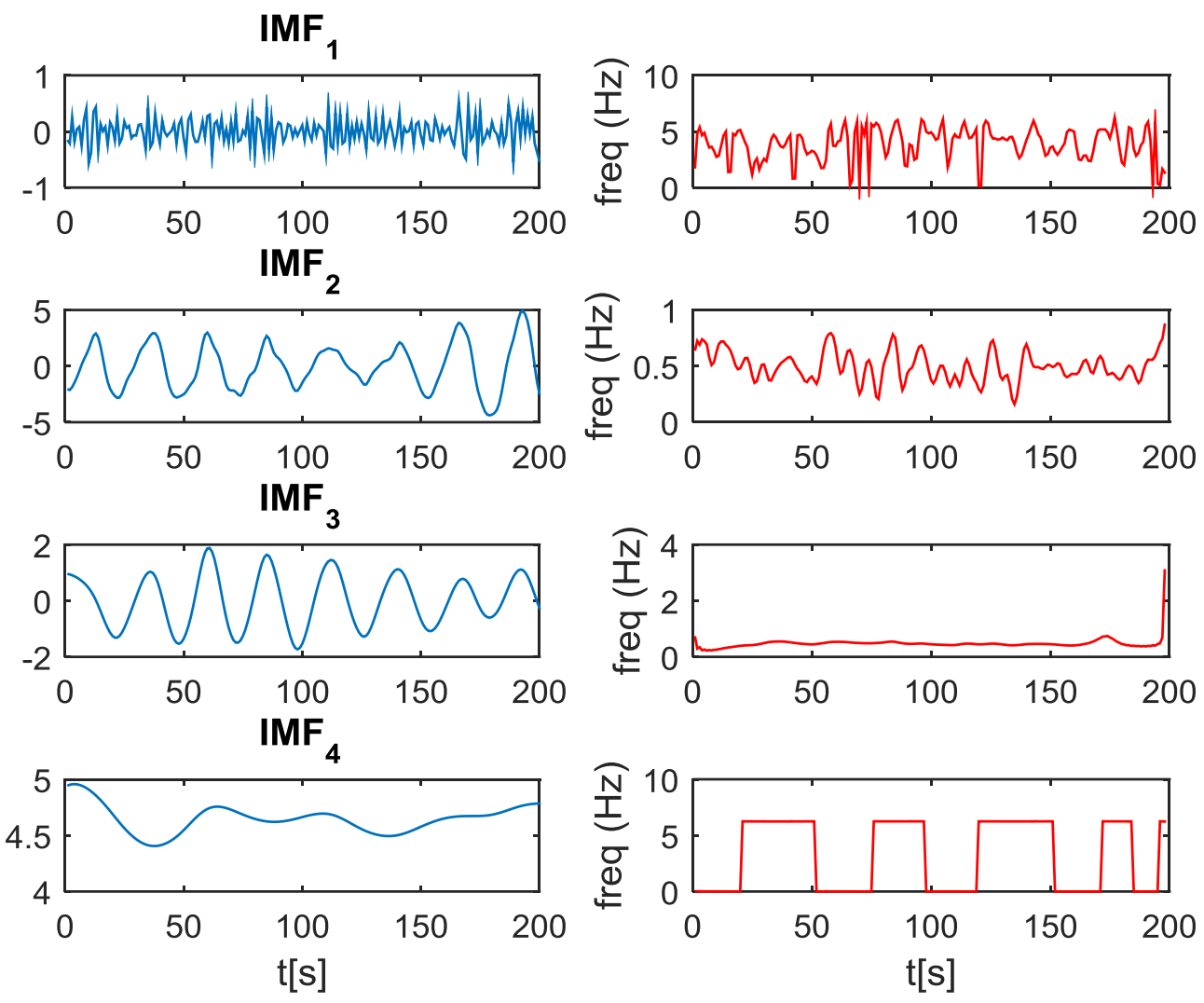

Figure 19. EEMD of real BWR signal along the IFs of each IMF.

Figure 20 shows the CEEMDAN of the same LPRM 3 signal segment. The IFs of each IMF are now shown. The IF of the first 3 IMFs are high and linked to noise whereas the IF of IMF 4 is a frequency highly concentrated around $0.5 \mathrm{~Hz}$ and visually speaking, IMF 4 looks like a cyclic function. IMF 4 is linked to DW instability. IMF 4 looks like a clean oscillating function. Figure 21 shows the improved CEEMDAN of the same LPRM 3 signal segment. the IFs of IMFs 1 and 2 is high (linked to noise) and oscillates around $5 \mathrm{H}$. The IF of IMF 3 oscillates around $0.5 \mathrm{~Hz}$ and IMF 3 looks like a cyclic oscillating function (this is the IMF linked to DW instability). The IF of IMF 4 is a low frequency component close to $0.3 \mathrm{~Hz}$. Visually speaking IMF 4 grants no information about the instability of the BWR. Improved CEEMDAN was the method that we chose to build BWR instability methodologies for uni variate signal analysis due to the fact that this EMD expansion is better that its predecessors and because the extracted IMFs linked to DW phenomena look clean and the oscillating function looks in our eyes, clean (the IMFs linked to the DW look visually like clean cosine functions of $0.5 \mathrm{~Hz}$ ). The HHT is a powerful tool to reveal frequency content of a signal in a time-frequency plane. Any rapid change in the time domain signal will impact its frequency. The HHT is able to capture that sudden change whereas Fourier transform is unable to accommodate that sudden signal behavior. With the HHT we no longer feel trap with assumptions regarding linearity of the studied signals. With the HHT, the frequency analysis of a signal becomes more realistic. 

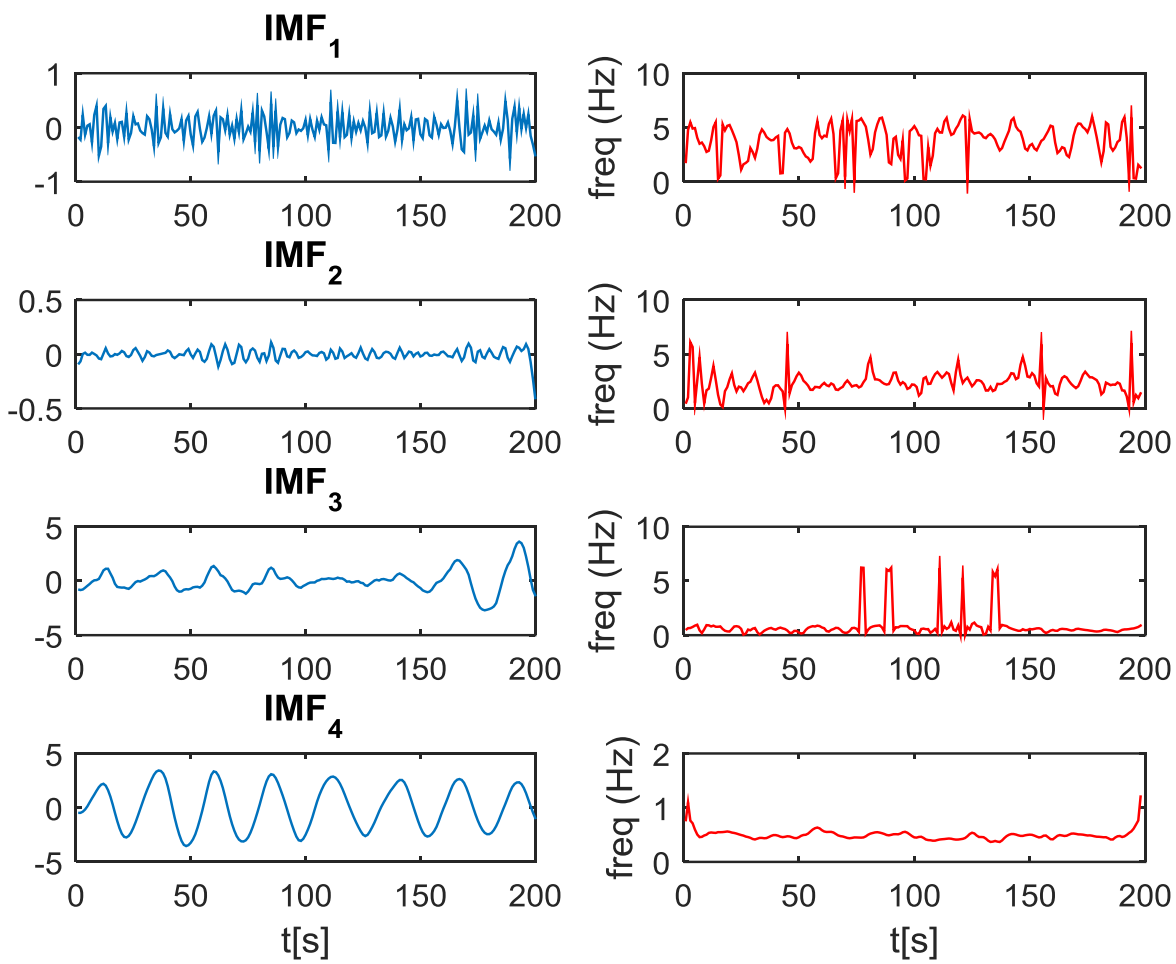

Figure 20. CEEMDAN of real BWR signal along the IFs of each IMF.
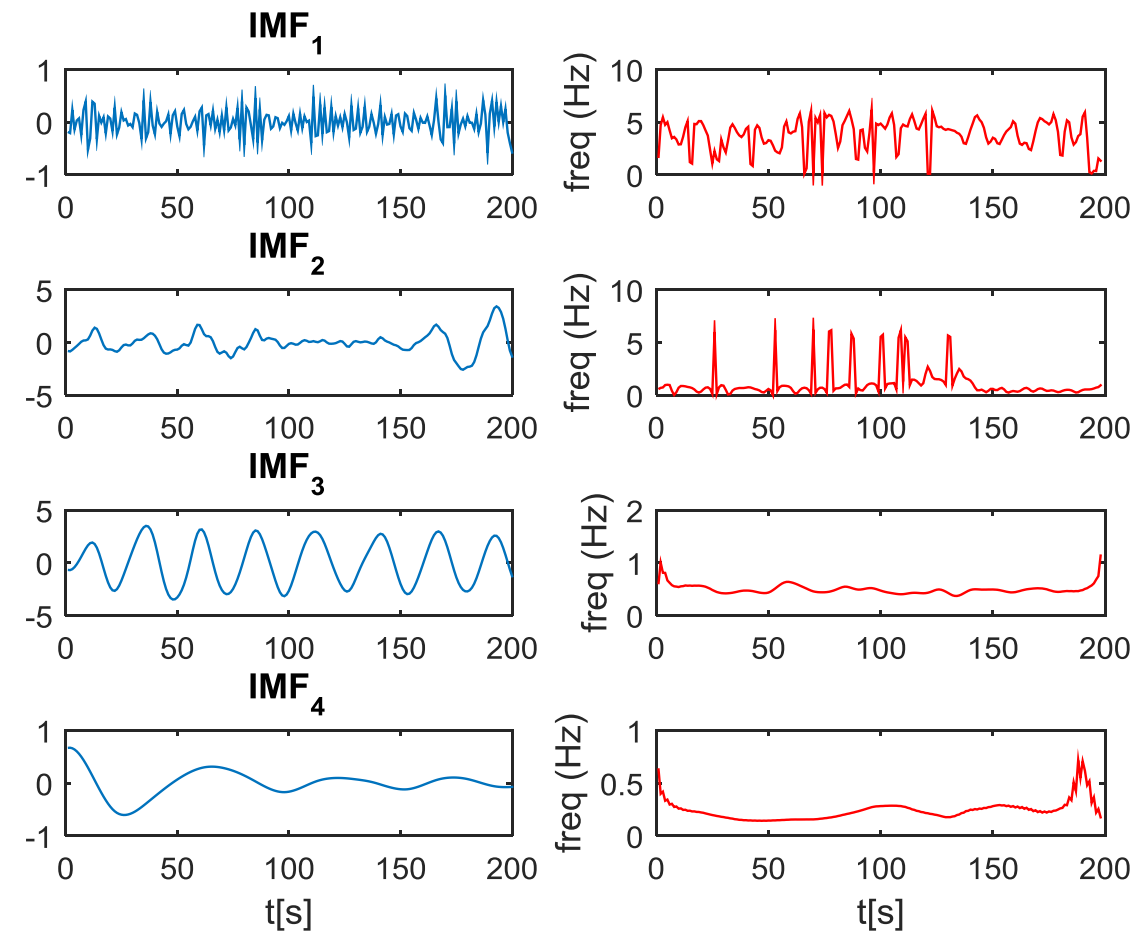

Figure 21. Improved CEEMDAN of real BWR signal along the IFs of each IMF. 


\subsection{The noise assisted multivariate empirical mode decomposition}

The multivariate empirical mode decomposition (MEMD) is a technique that was proposed by Rehman and Mandic, 2009 to make the default EMD suitable for processing of multichannel signals. To shed further light in the performance of the MEMD method, its behavior was analyzed in the presence of white Gaussian noise (Rehman and Mandic, 2011) and it was found that, similarly to EMD. The developed MEMD also in essence acts as a dyadic filter bank on each channel of the multivariate input signal, such MEMD property is illustrated in Figure 22 and its algorithm is given below. However, unlike EMD, the MEMD better aligns the corresponding IMFs from different channels across the same frequency range which is crucial for real world applications and from such studies, the noise assisted multivariate empirical mode decomposition (NA-MEMD) emerged to help fix the mode mixing issue of the MEMD (the MEMD is the multivariate expansion of EMD).

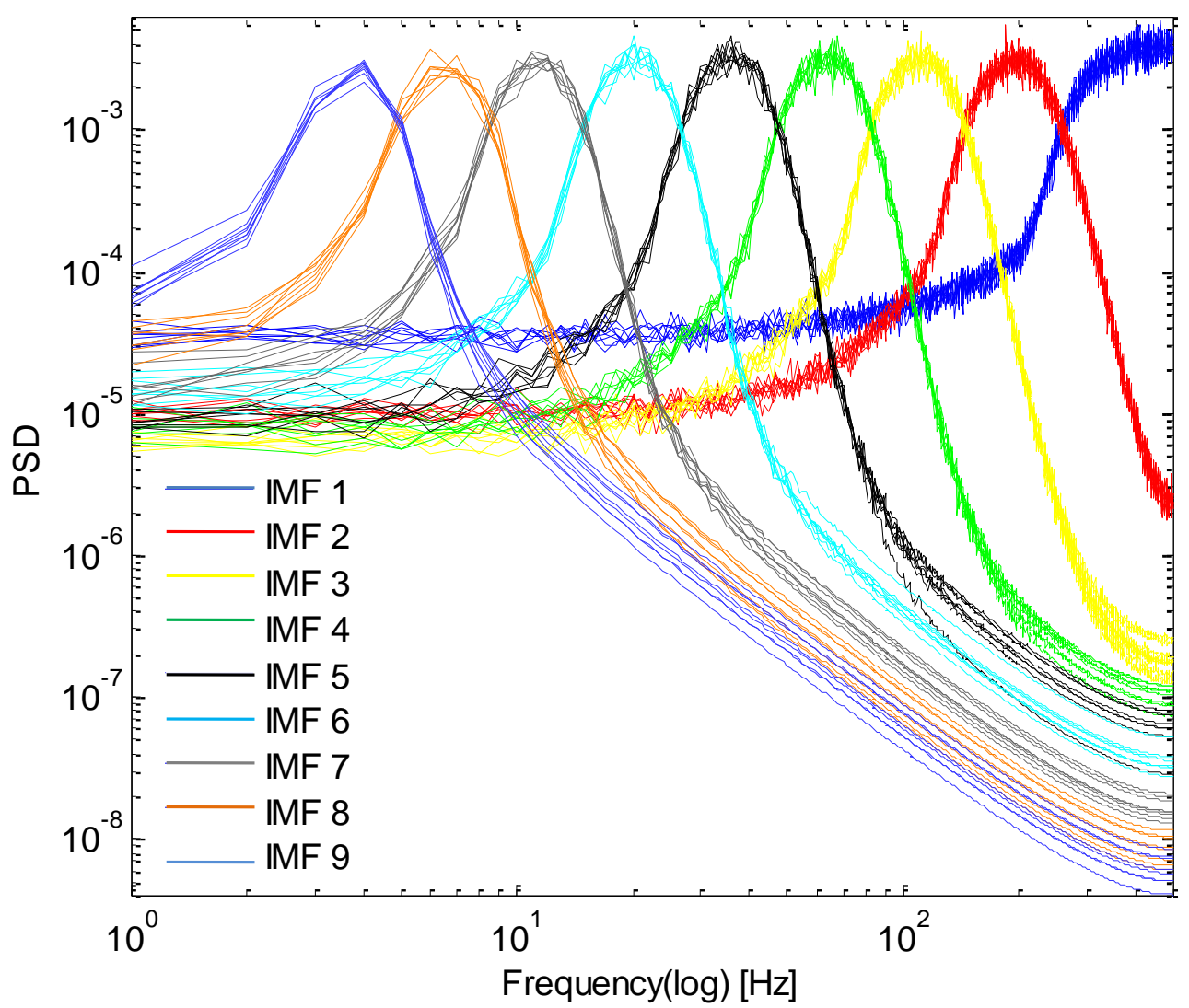

Figure 22. Averaged spectra of IMFs (1-9) obtained for 50 realizations of eight-channel white Gaussian noise via MEMD. 
The NA.MEMD method which makes use of the quasi-dyadic filter bank properties of MEMD on white noise, it is capable of significantly reducing the mode mixing problem for classes of signals where the quasi-dyadic filter bank structure proves useful. Embarking upon the quasi-dyadic filter bank structure of standard EMD for broadband noise, many EMD variants were proposed, in which multiple realizations of white noise were added to the input signal before being decomposed via EMD. This helps to establish a uniformly distributed reference scale which, in turn, results in corresponding IMFs exhibiting a quasidyadic filter bank structure. Following the latter idea, to explore the benefits of the quasidyadic filter bank structure of the default MEMD on white noise. In the NA-MEMD (Rehman and Mandic, 2011) a total of $m$ extra independent channels containing white noise are added in the MEMD decomposition of the multivariate signal of interest to exploit such interesting benefits of this filter bank property. The extracted IMFs corresponding to the $m$ channels of white noise are then discarded yielding a set of IMFs linked with only the original input signal. Since the added noise channels occupy a broad range in the frequency spectrum, MEMD aligns its IMFs based on the quasi-dyadic filter bank, with each component carrying a frequency sub band of the original signal. In doing so, IMFs corresponding to the original input signal also align themselves according to the structure of the quasi-dyadic filter bank. This, in turn, helps to mitigate the mode mixing problem within the extracted IMFs. The details of the NA-MEMD method are as follows, but first let us introduce the steps of the classic MEMD method:

Method 5 (The multivariate empirical mode decomposition (MEMD)).

Consider a multivariate signal $\mathbf{v}(t)$ :

Consider a sequence of $N$ dimensional vectors $\{\mathbf{v}(t)\}_{t=1}^{T}=\left\{v_{1}(t), v_{2}(t), \ldots, v_{N}(t)\right\}$ representing a multivariate signal with $N$ components, and $\mathbf{x}^{\theta_{k}}=\left\{x_{1}^{k}, x_{2}^{k}, \ldots, x_{N}^{k}\right\}$ denoting a set of direction vectors along the direction given by angles $\theta^{k}=\left\{\theta_{1}^{k}, \theta_{2}^{k}, \ldots, \theta_{N-1}^{k}\right\}$ on a (l-1) sphere. Then the extraction of the first IMF from the given MEMD steps is summarized in next steps:

Step 1. Generate the point set based on the Hammersley sequence for sampling on an (l-1) sphere (Niederreiter, 1992).

Step 2. Calculate a projection, denoted by $\left.p^{\theta_{k}}(t)\right\}_{t=1}^{T}$, of the input multivariate signal $\{\mathbf{v}(t)\}_{t=1}^{T}$ along the direction vector $\mathbf{x}^{\theta_{k}}$, for all $k$ (the whole set of direction vectors), giving $p^{\theta_{k}}(t)_{k=1}^{K}$ as the set of projections.

Step 3. Find the time instants $\left\{t_{i}^{\theta_{k}}\right\}_{k=1}^{K}$ corresponding to the maxima of the set of projected signals $\left.p^{\theta_{k}}(t)\right\}_{k=1}^{K}$.

Step 4. Interpolate $\left[t_{i}^{\theta_{k}}, \mathbf{v}\left(t_{i}^{\theta_{k}}\right)\right]$, for all values of $k$, to obtain multivariate envelope curves $\left.\mathbf{e}^{\theta_{k}}(t)\right\}_{k=1}^{K}$. 
Step 5. For a set of $K$ direction vectors, calculate the mean $\mathbf{m}(t)$ of the envelope curves as

$$
\mathbf{m}(t)=\frac{1}{K} \sum_{k=1}^{K} \mathbf{e}^{\theta_{k}}(t)
$$

Step 6. Extract the detail $c(t)$ using $c(t)=x(t)-m(t)$. If the detail $c(t)$ fulfills the stoppage criterion for a multivariate IMF, apply the above procedure to $x(t)-c(t)$, otherwise apply it to $c(t)$.

Once the first IMF (or mode) is extracted, it is subtracted from the input signal and the same process (steps from method 5) is applied to the resulting signal yielding the second IMF and so on, the process is repeated until all the IMFs are extracted and only a residue is left; in the multivariate case, the residue corresponds to a signal whose projections do not contain enough extrema to form a meaningful multivariate envelope. The sifting process for a multivariate IMF can be stopped when all the projected signals fulfill any of the stoppage criteria adopted in the conventional EMD. Now, that the steps of the MEMD method have been given, the NA-MEMD is calculated as:

Method 6 The noise assisted multivariate empirical mode decomposition (NA-MEMD)

Step 1. Create an uncorrelated Gaussian white noise time-series (m-channel) of the same length as that of the input.

Step 2. Add the noise channels (m-channels) created in step 1 to the input multivariate $(N$-channels) signal, obtaining an $(N+m)$-channel signal.

Step 3. Process the resulting $(N+m)$-channel multivariate signal using the MEMD algorithm (listed above), to obtain multivariate IMFs.

Step 4. From the resulting $(N+m)$-variate $I M F s$, discard the $m$ channels corresponding to the noise, giving a set of N-channel IMFs corresponding to the original signal.

It should be mentioned that the noise assisted methods (EEMD, CEEMDAN, improved CEEMDAN and the multivariate NA-MEMD) for mitigating the mode mixing problem are expected to be most useful for signals in which the dyadic filter bank decomposition is relevant. This is the case for the studied BWR signals. To illustrate the mode alignment property of the MEMD, a synthetic hexavariate time series is explored; each component (variate), is shown in the top row of Figure 23 ( denoted by X,Y and Z), such signal was constructed from a set of four sinusoids. the component $\mathrm{X}$ is made by the sum of the four sinusoids of frequency modes: $2 \mathrm{~Hz}, 8 \mathrm{~Hz}, 16 \mathrm{~Hz}$ and $32 \mathrm{~Hz}$. The component $\mathrm{Y}$ is made by the sum of two sinusoids of frequency modes: $16 \mathrm{~Hz}$ and $8 \mathrm{~Hz}$ and the last component $\mathrm{Z}$ is made by the sum of two sinusoids of frequency modes: $8 \mathrm{~Hz}$ and $2 \mathrm{~Hz}$. The MEMD was applied to this decompose this signal yielding multiple IMFs shown in Figure 23. Observe 
that the sinusoid common to all the components of the input is the third IMF, whereas the remaining three frequency modes were also accurately extracted in the respective IMF decomposition level. Such mode alignment cannot be achieved by the real-valued EMD applied component-wise, as it generally does not yield the same number of IMFs per component.
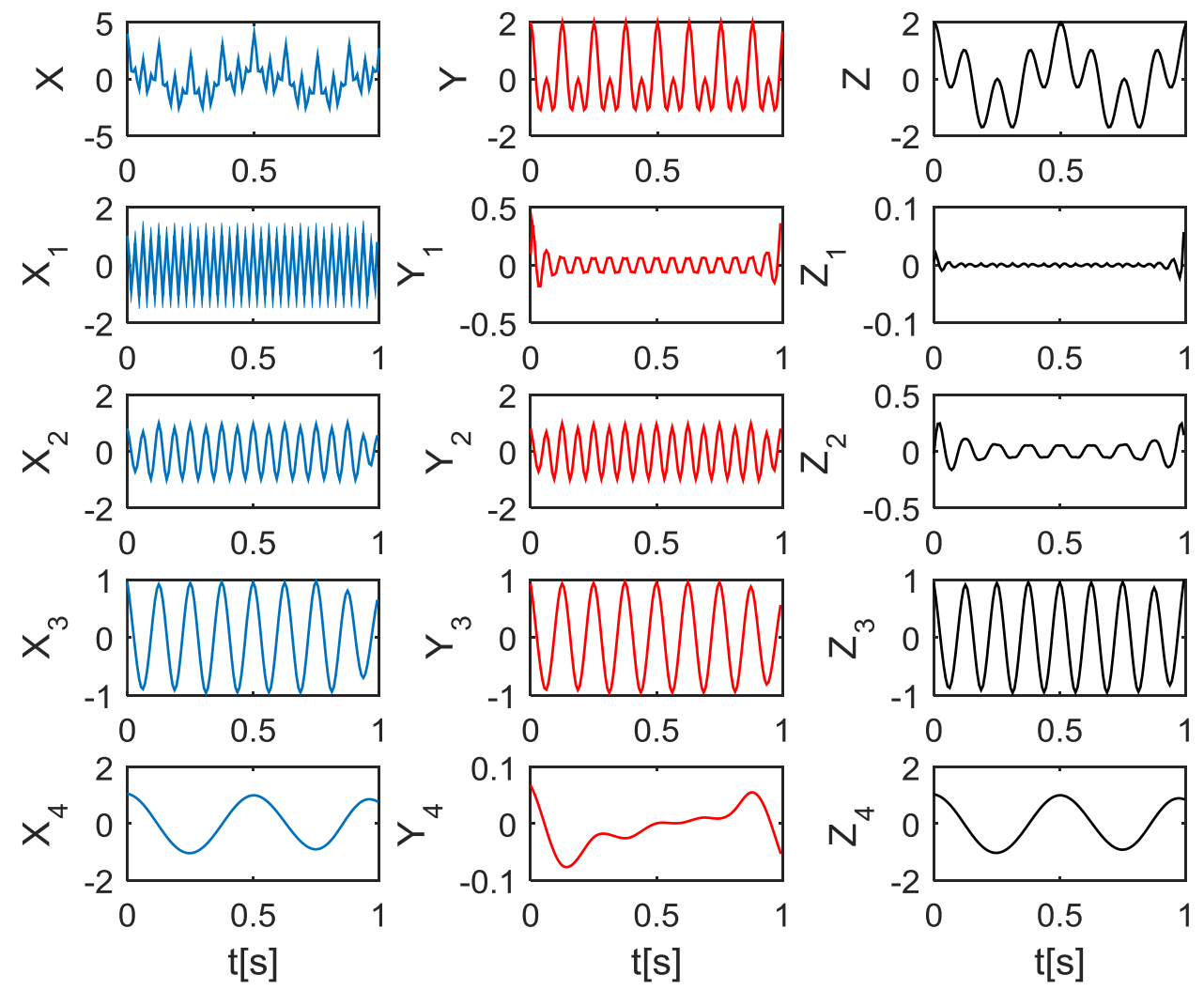

Figure 23. Decomposition of a synthetic multivariate signal (X, Y, Z) exhibiting frequency modes (with $\mathrm{f} 1=2 \mathrm{~Hz}, \mathrm{f} 2=8 \mathrm{~Hz}, \mathrm{f} 3=16 \mathrm{~Hz}$ and $\mathrm{f} 4=32 \mathrm{~Hz}$ ) via the MEMD. Each IMF carries a single frequency mode, illustrating the alignment of common scales within different components of a multivariate signal.

The multivariate EMD (MEMD) has been applied in other engineering applications, such as: in the classification of motor imagery brain computer interface (Park et al., 2012), in soil water prediction ( $\mathrm{Hu}$ and $\mathrm{Si}, 2013$ ) and for quantifying multivariate phase synchronization (Mutlu and Aviyente, 2011).

Now, in order to illustrate the capabilities of the noise assisted MEMD (NA-MEMD). Consider a synthetic signal consisting of a combination of two different tones of low frequency, one tone is added to the other in seconds 24 to 31 . The resulting signal is shown in the top of Figure 24. Also shown in Figure 24 are the IMFs obtained from applying default EMD to the signal; mode mixing is evident since IMF 1 contains multiple modes, 
the Original signal could not be separated successfully by default EMD. We next processed the same signal using the NA-MEMD method with two extra noise channels $(m=2)$.

The IMFs from the resulting trivariate signal are shown in Figure 25. Observe that the IMFs corresponding to the first channel are now free from mode mixing, as all the tones are decomposed as separate IMFs (IMF 5 and IMF 6, respectively). The IMFs related to noise are later discarded (IMFs of noise 1 and IMFs of noise 2). In the NA-MEMD technique, the number of noise channels $m$ and the amplitude of noise channels must be chosen so that the desired dyadic filter bank structure of the MEMD is exploited. In the following example the NA-MEMD is tested with real BWR signals that stem from the Ringhals stability benchmark (Lefvert, 1996).
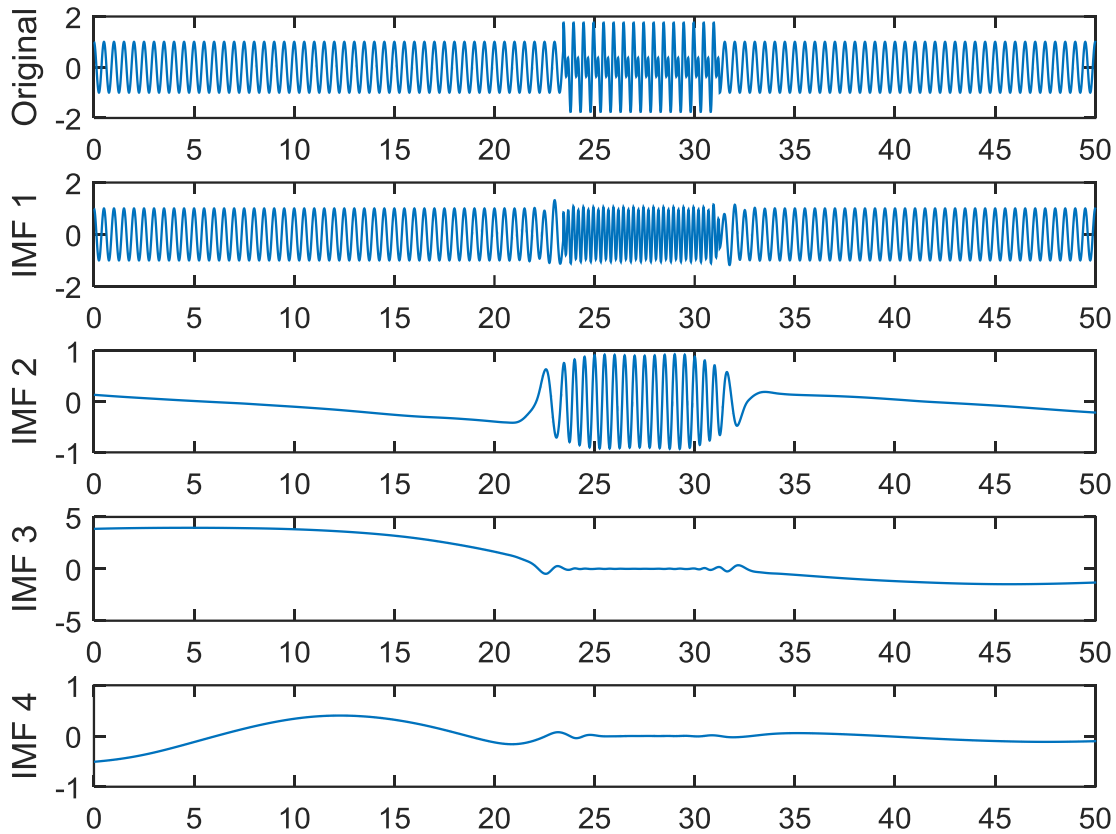

Figure 24. IMFs of a synthetic signal obtained by applying default EMD. Mode mixing is evident in IMF 1, the Original signal could not be separated by default EMD. The two tones are still added together in IMF 1. 

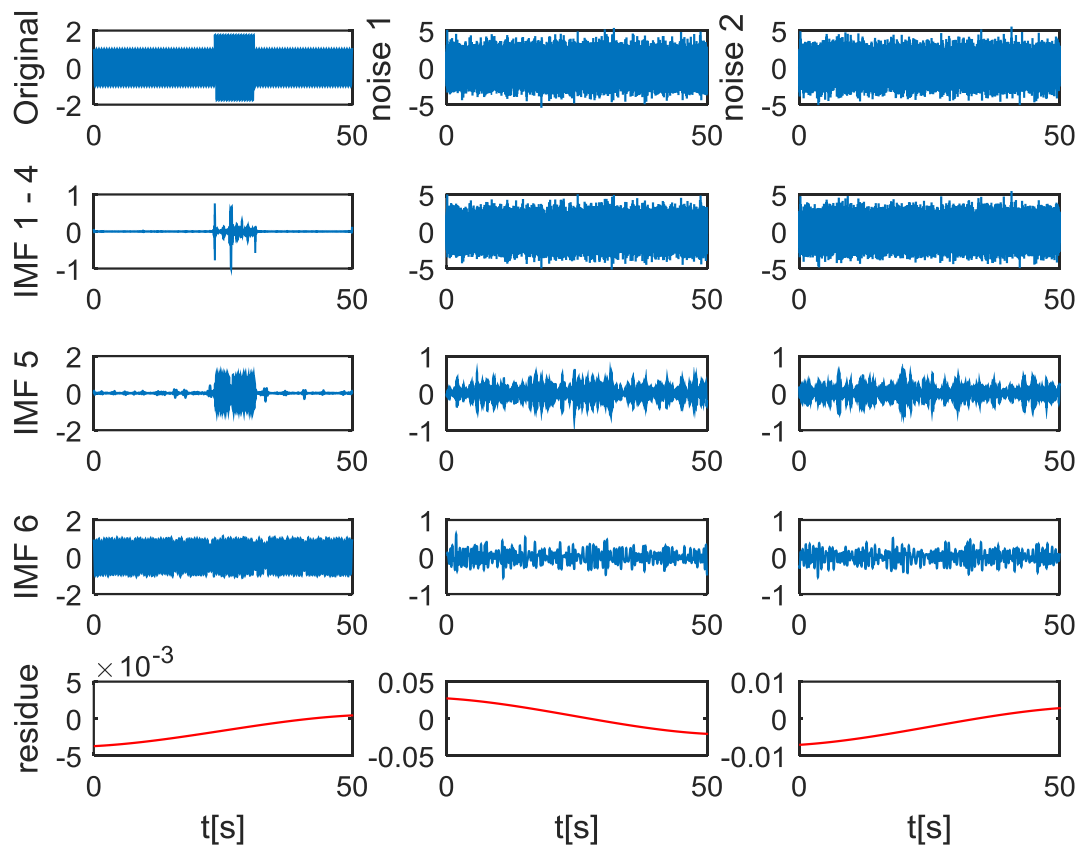

Figure 25. IMFs of a synthetic signal obtained by NA-MEMD (left hand column); the IMFs of the two noise channels shown in the middle and right hand column. Mode mixing observed in IMFs from default EMD is significantly reduced with IMF 5 and IMF 6 containing the two original tones.

Now, we decompose 3 LPRM segments of $15 \mathrm{~s}$ of time span each with the NA-MEMD. The chosen LPRMs belong to case 9 cycle 14 of the Ringhals stability benchmark and are labeled as LPRM 1, LPRM 2 and LPRM 3. Figure 26 shows the results of the NA-MEMD decomposition of the 3 LPRM segments. The original LPRM signals are shown on the top row this figure. The IMFs 1-3 of each channel were added together (such IMFs are linked to acquisition noise), The IMFs 4 exhibit a cyclic oscillating waveform (such IMFs 4 are related to the Density Wave instability), IMFs 5 and 6 are also shown and their content is irrelevant to our application. 

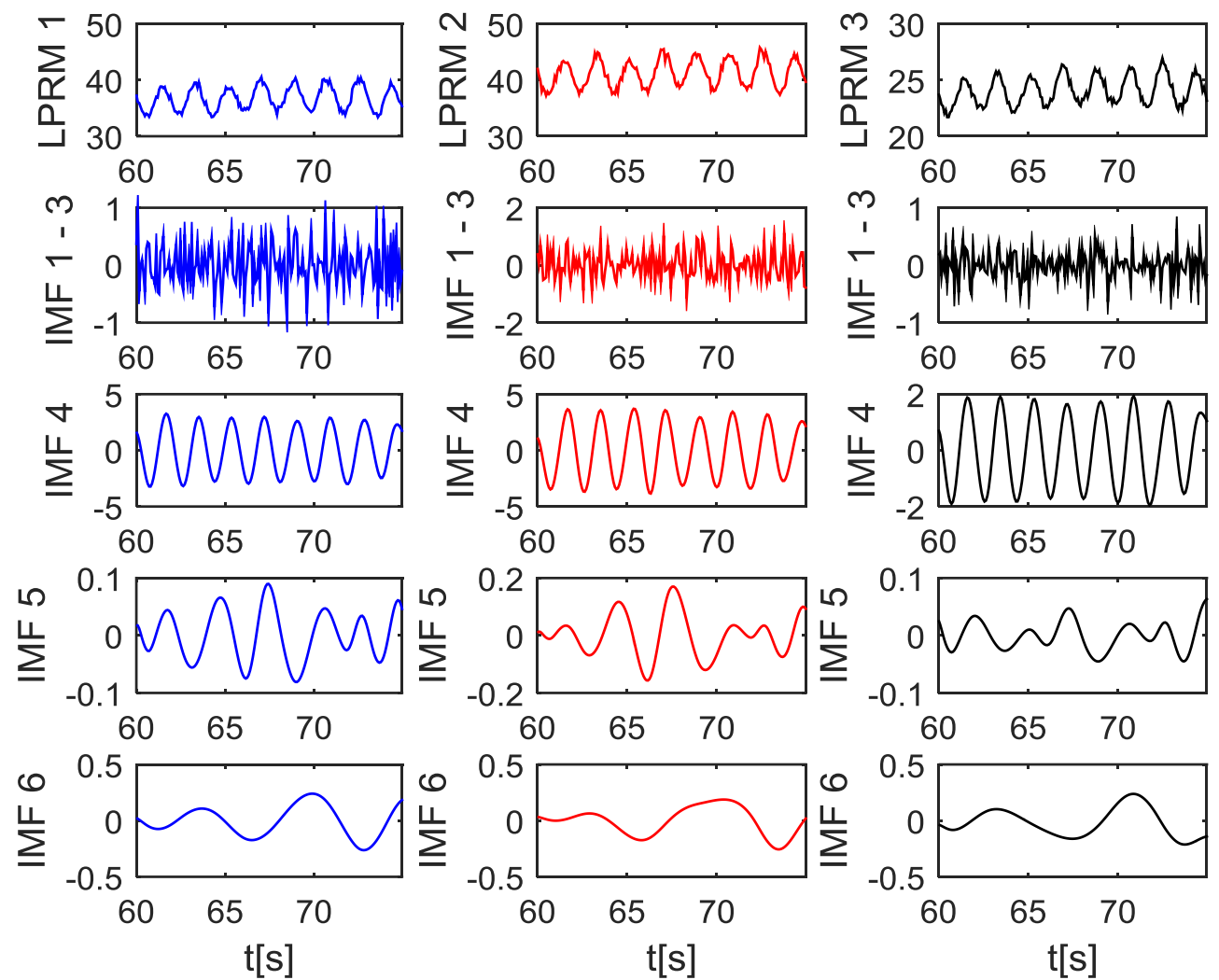

Figure 26. IMFs of 3 LPRM segments of $15 \mathrm{~s}$ of time span each extracted by NA-MEMD. IMFs 1-3 of each channel are added together (IMFs 1-3 associated to acquisition noise). IMFs 4 are linked to the DW instability issue because they look visually like cyclic oscillating waveforms. IMFs 5 and 6 are also shown and their content is irrelevant to the detection of the DW instability. A total of 8 IMFs were extracted for each LPRM.

Now, Figure 27 - Figure 29 shows the first four extracted IMFs from each LPRM (LPRM 1 to 3). The instantaneous frequencies of extracted IMFs are also shown in these Figures (all the IFs are computed by means of HHT). The IFs of IMFs 1 to 3 are high and related to acquisition noise whereas the IF of IMF 4 is a line oscillating around $0.5 \mathrm{~Hz}$. IMFs number 4 are the IMFs linked to the density wave (DW) instability. The NA-MEMD is the technique that is chosen to analyze multichannel BWR signals from various LPRMs. The NA-MEMD can also be applied to decompose a uni variate signal if many noise channels are added to the ensemble. The NA-MEMD decomposes multi channel non-stationary signals that stem from non-linear sources in a data driven way. With the HHT it is possible to analyze the frequency content of each IMF. 

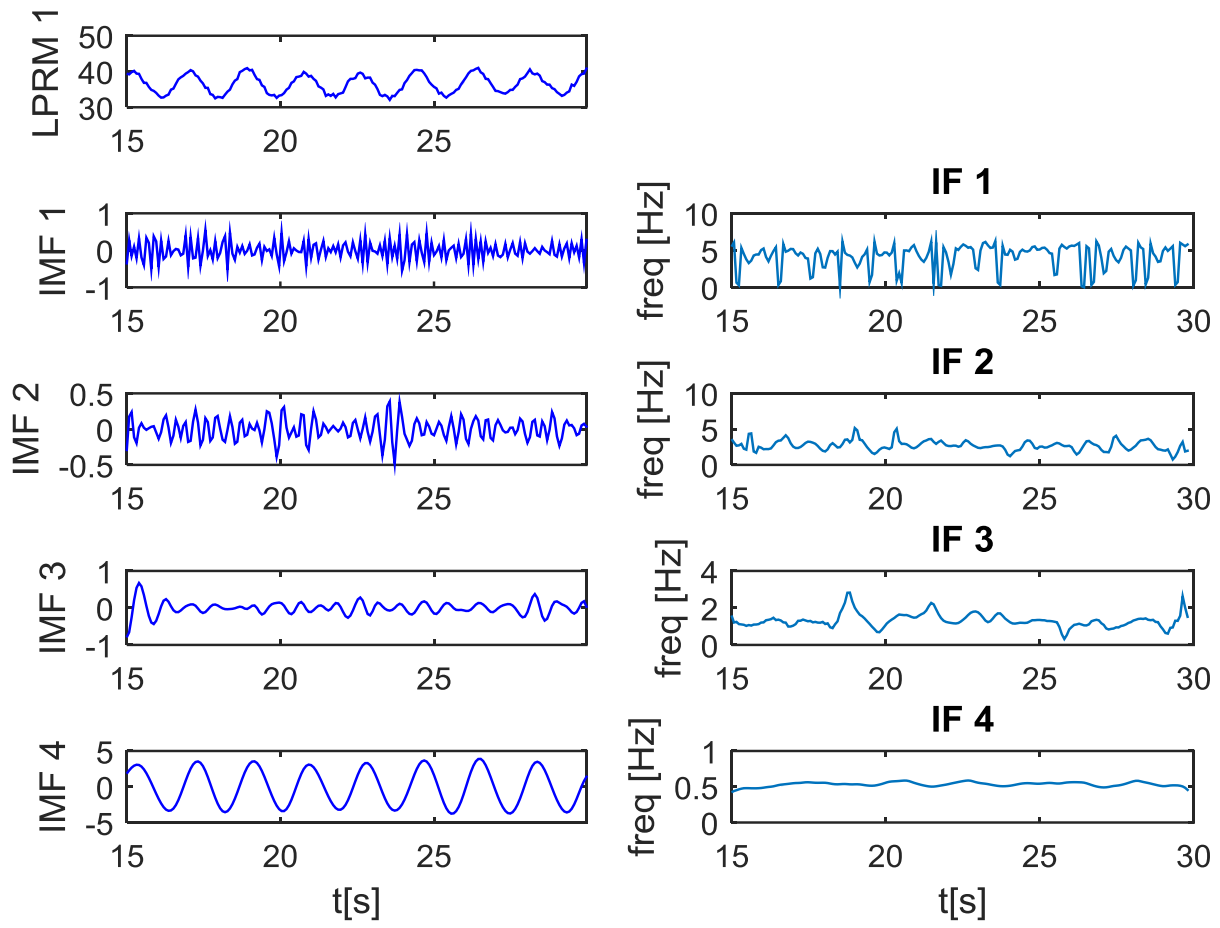

Figure 27. Extracted IMFs of LPRM 1 with their respective IFs (computed by means of HHT). IMF 4 is the IMF linked to the density wave instability.
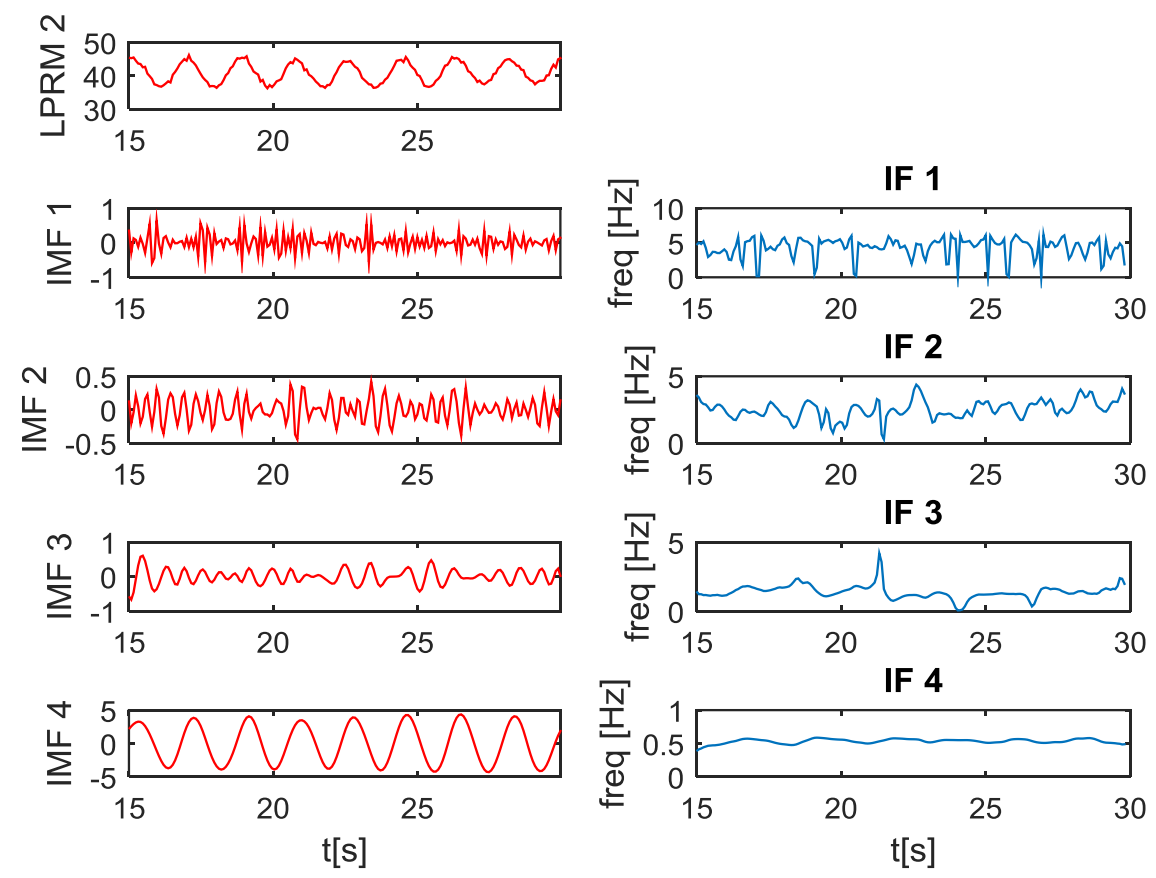

Figure 28. Extracted IMFs of LPRM 2 with their respective IFs (computed by means of HHT). IMF 4 is the IMF linked to the density wave instability. 

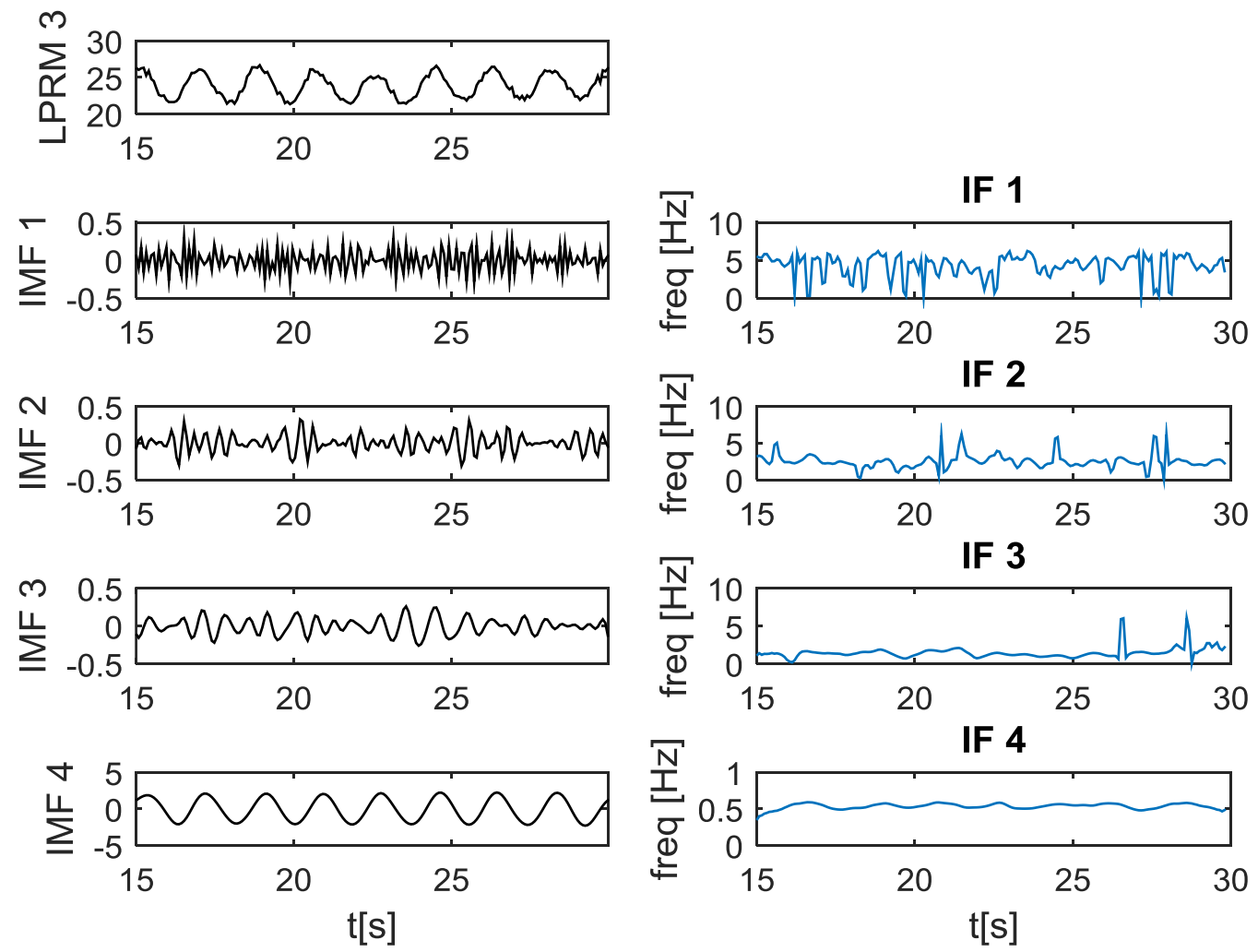

Figure 29. Extracted IMFs of LPRM 3 with their respective IFs (computed by means of HHT). IMF 4 is the IMF linked to the density wave instability. 


\section{Chapter 4}

\section{Non-linear behavior of a BWR}

\subsection{A non-linear reduced order model (ROM)}

Reduced Order Models are used to study system stability and its dynamics. They are usually obtained by averaging over time and/or space, and often represent a system by a set of non-linear ordinary differential equations (ODEs). In the nuclear engineering discipline, the basic point reactor kinetics model needs to be extended to capture the effects of several feedback mechanisms that play a significant role in reactor dynamics. For instance, changes in reactor power lead to changes in core component temperature and void fraction, which in turn have an impact on the reactivity. A simple but powerful model which has been extensively used for BWR stability analysis was developed by March-Leuba, 1986, such non-linear reduced order model (ROM) represents qualitatively the BWR dynamics, the complex (i.e., chaotic) dynamics of BWR unstable behavior are also captured by this model. The studied ROM is given by the next set of differential equations:

$$
\begin{gathered}
\frac{d n(\mathrm{t})}{d t}=\frac{\rho(\mathrm{t})-\beta}{\Lambda} n(t)+\lambda c(\mathrm{t})+\frac{\rho(t)}{\Lambda} \\
\frac{d c(t)}{d t}=\frac{\beta}{\Lambda} n(t)-\lambda c(\mathrm{t}) \\
\frac{d T(\mathrm{t})}{d t}=a_{1} n(\mathrm{t})-a_{2} T(t) \\
\frac{d^{2} \rho_{\alpha}(\mathrm{t})}{d t^{2}}+a_{3} \frac{d \rho_{\alpha}(\mathrm{t})}{d t}+a_{4} \rho_{\alpha}(t)=\kappa k_{o} T(\mathrm{t}) \\
\rho(t)=D T(t)+\rho_{\alpha}(\mathrm{t})
\end{gathered}
$$

Here the variables $n(t)$ and $c(\mathrm{t})$ are converted by the following equations as fluctuations caused from the equilibrium values $N_{o}$ and $C_{o}$ of the steady state:

$$
\begin{gathered}
n(t)=\frac{N(\mathrm{t})-\mathrm{N}_{o}}{N_{o}} \\
c(t)=\frac{c(t)-C_{o}}{N_{o}}
\end{gathered}
$$


where $n(\mathrm{t})$ is the excess neutron population normalized to the steady state neutron population, $c(t)$ is the excess delayed neutron precursors concentration also normalized to the steady state neutron population; $T(t)$ is the excess average fuel temperature; and $\rho_{\alpha}(\mathrm{t})$. Since we are interested in the non-linear region above the threshold for linear stability, the indicators for the base case were calculated from a fit to the LAPUR transfer function for test $7 \mathrm{~N}$ of the Vermont Yankee reactor, the value of the indicators is given in the following Table 5:

Table 5. Mode indicators for the Vermont Yankee test 7 N.

\begin{tabular}{ccc}
\hline Model coefficient & Value & Units \\
\hline$a_{1}$ & 25.94 & $\mathrm{Ks}^{-1}$ \\
$a_{2}$ & 0.23 & $\mathrm{~s}^{-1}$ \\
$a_{3}$ & 2.25 & $\mathrm{~s}^{-1}$ \\
$a_{4}$ & 6.82 & $\mathrm{~s}^{-2}$ \\
$\kappa_{o}$ & $-3.70 \times 10^{-3}$ & $\mathrm{~K}^{-1} \mathrm{~s}^{-2}$ \\
$\mathrm{D}$ & $-2.52 \times 10^{-3}$ & $\mathrm{~K}^{-1}$ \\
$\beta$ & 0.0056 & - \\
$\lambda$ & 0.08 & $\mathrm{~s}$ \\
$\Lambda$ & $4 \times 10^{-5}$ & $\mathrm{~s}^{-1}$ \\
\hline
\end{tabular}

The indicator $\kappa$ is related to the void reactivity coefficient and the fuel heat transfer coefficient. $\kappa$ controls the feedback gain, defining the stability of this reactor model. The value $(\kappa=1)$ is the critical value at which the model becomes unstable. By artificially increasing the feedback gain $\kappa>1$ we can make the model unstable and under these conditions, we can study the model behavior in the non-linear region. For instance, Figure 30 shows a stable reactor output $n(t)$ for $\kappa=0.75$ and its reactivity value $\rho_{\alpha}$. This is an example of a stable nuclear reaction; which means that the number of neutrons remains constant. If the fission reaction is kept stable: It is said that the BWR is critical. Ideally speaking the $\rho_{\alpha}$ of a stable fission reactor should be zero. In this example, $\rho_{\alpha}$ is in the order of $10^{-6}$.

As soon as $\kappa$ starts to increase to approach the stability threshold point $(\kappa=1)$ the reactor output $n(\mathrm{t})$ starts to show signs of cyclic behavior (as if it started to look like a cosine function). The reactivity value $\rho_{\alpha}$ becomes bigger. The moment $\rho_{\alpha}$ becomes positive, the reactor becomes unstable. The fission reactions are growing inside the core and the number 
of neutrons is increasing beyond control. We say in the BWR jargon that the reactor is becoming supercritical.

Figure 31 shows another stable reactor output $n(t)$ for $\kappa=0.95$ and its reactivity value $\rho_{\alpha}$. In this example, we are approaching the stability threshold point $(\kappa=1)$; which means that the number of neutrons is starting to increase. $n(t)$ begins to look like a damped oscillation. The ROM is still stable and the reactivity $\rho_{\alpha}$ has increased and now is in the order of $10^{-4}$.

Figure 32 shows a marginally stable solution of this ROM for $\kappa=1$ (The ROM solution is now located on the threshold of instability). $n(t)$ looks like a cyclic function now (and no longer damped). reactivity $\rho_{\alpha}$ exhibits cyclic behavior too. The emerging $\rho_{\alpha}$ oscillation is still too small (in the order of $10^{-4}$ ). But, $\rho_{\alpha}$ is nevertheless positive. The first signs of BWR instability are beginning to appear. The number of neutrons is beginning to increase.
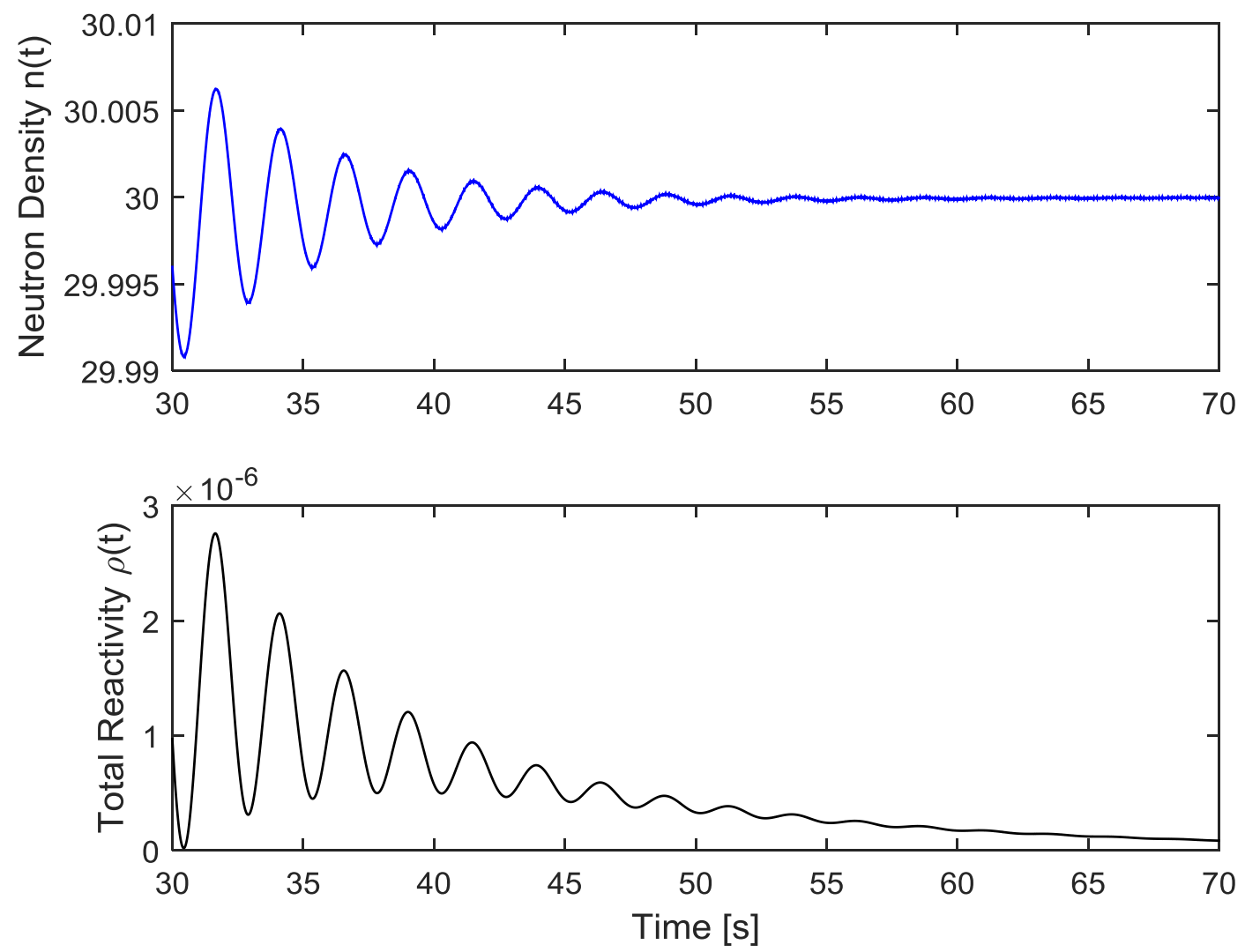

Figure 30. Stable ROM output for $\kappa=0.75$. 

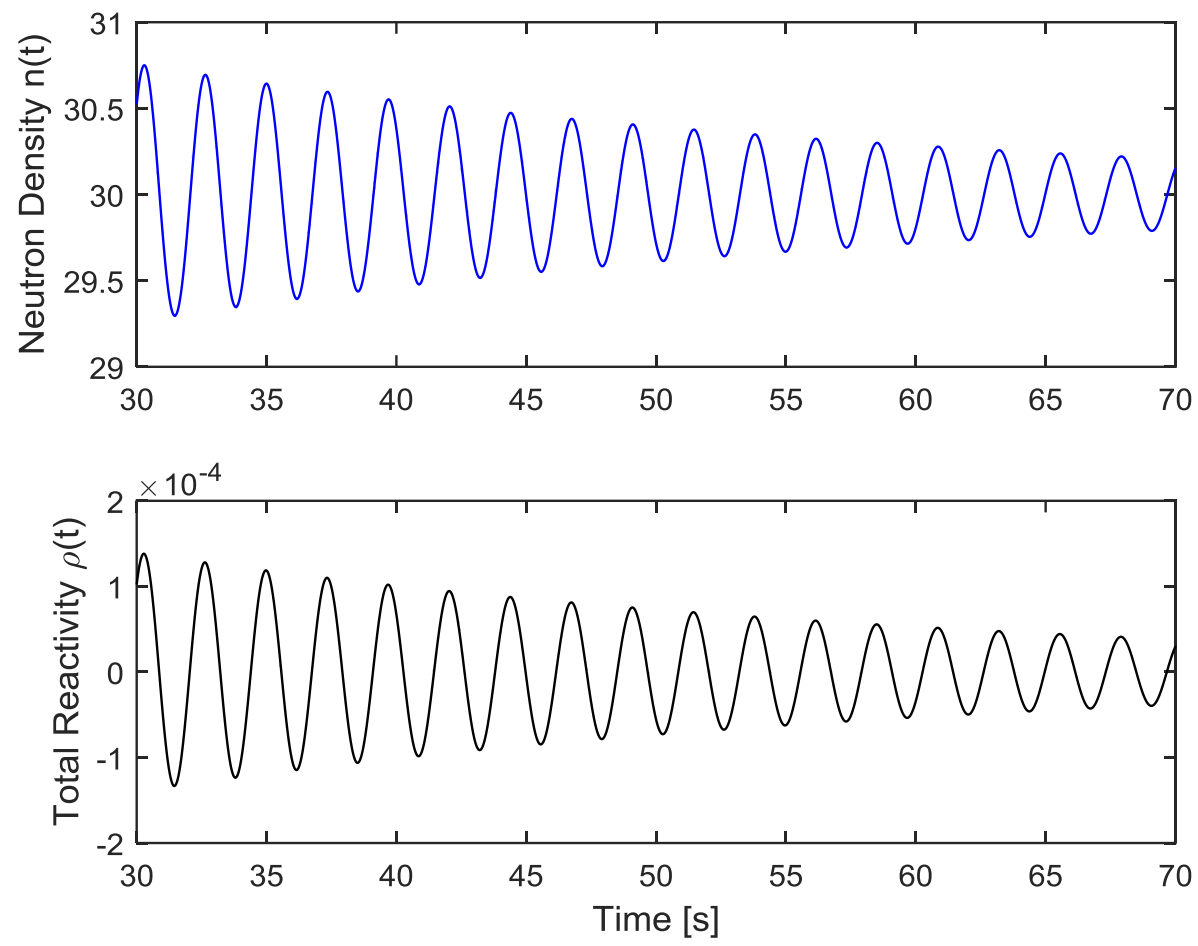

Figure 31. Stable ROM output for $\kappa=0.95$.
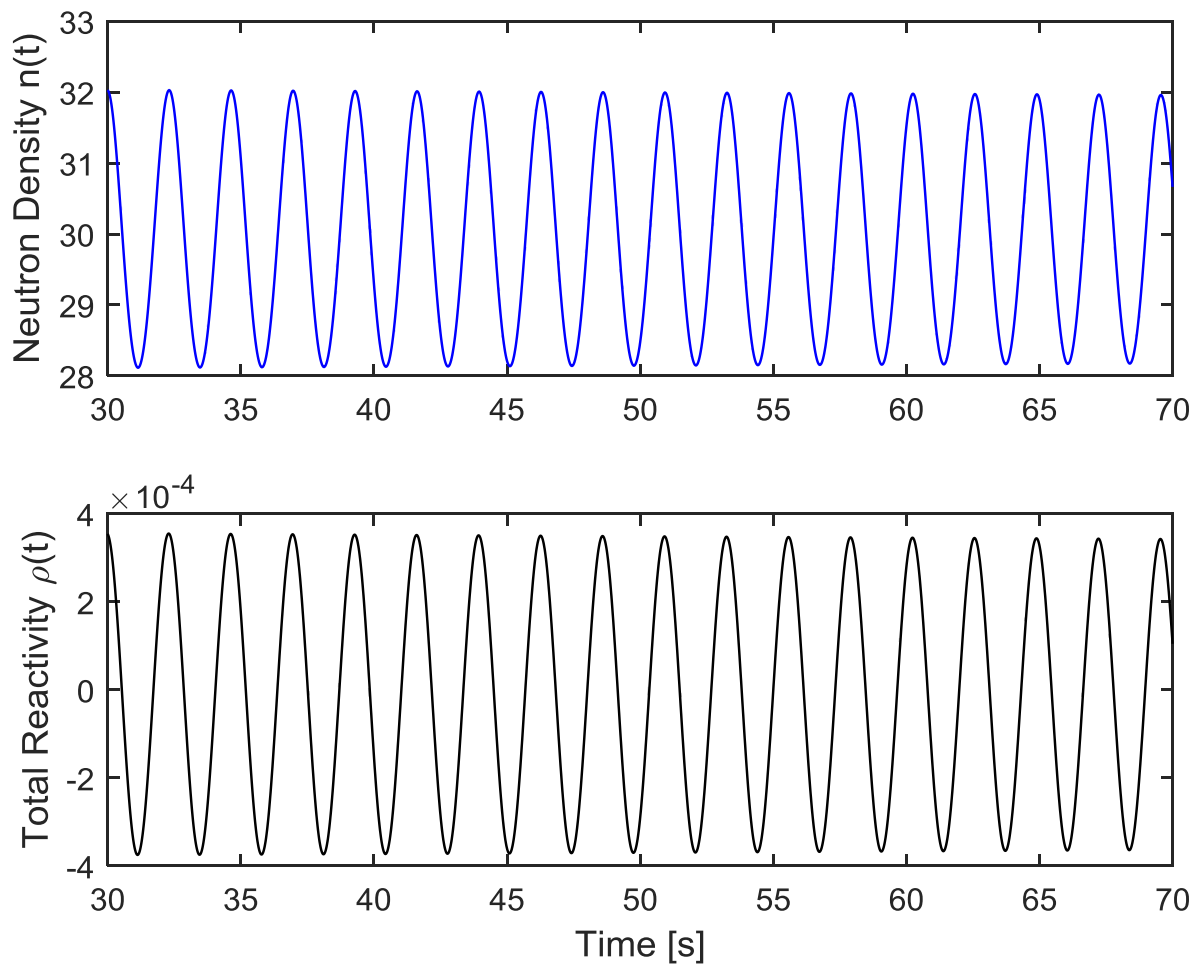

Figure 32. Marginally stable solution of the ROM for $\kappa=1$. 
Figure 33 now shows an unstable reactor output $n(t)$ for a $\kappa=1.05$. The oscillation visually looks like a cyclic oscillation. But now, the oscillation is increasing with time. The reactivity $\rho_{\alpha}$ shows a similar behavior to $n(t) . \rho_{\alpha}$ is positive and in the order of $10^{-3}$. The fission reactions are increasing within the core with no control.
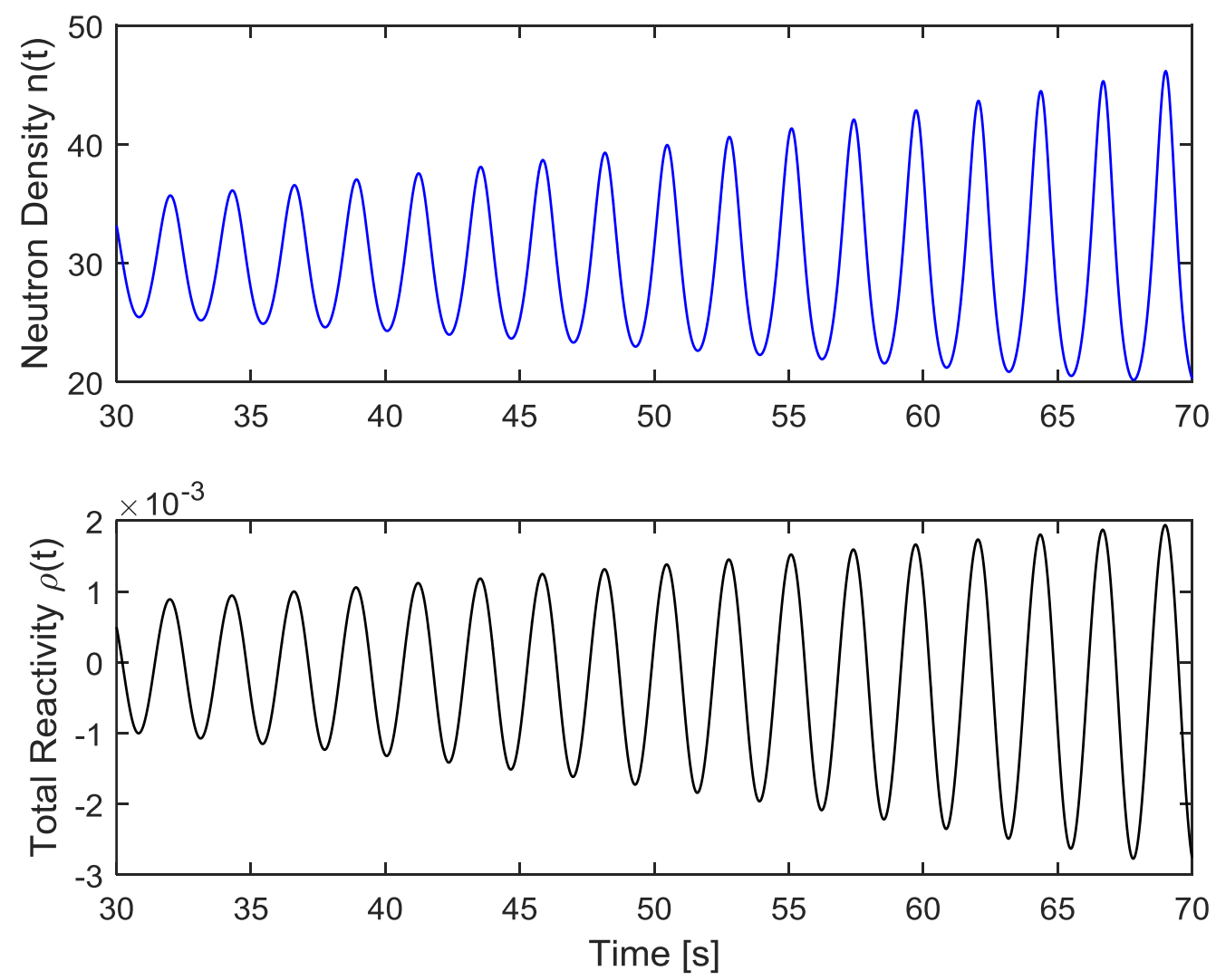

Figure 33. Unstable ROM output for $\kappa=1.05$.

In all these simulations, the initial condition vector is kept at $[0.1,0.1,1,0,0]$. The numerical integration method is the standard Runge-Kutta 4th order method and the integration step is $\Delta t=0.01 \mathrm{~s}$. The first $30 \mathrm{~s}$ of all the example reactor outputs were removed to eliminate transients.

\subsection{Bifurcations}

March-Leuba, 1986 investigated the evolution of stable limit cycles and a series of period doublings, which eventually led to chaotic oscillations. Muñoz-Cobo and Verdú, 1991 used this ROM to study the limit cycle oscillations in BWR by applying the Hopf bifurcation theory. In the stable regime, the system is said to be stable if following a perturbation, the resulting oscillation is eventually damped and the solution of the system converges to the 
equilibrium state. Figure 30 shows an example for a stable system, for after a small perturbation the system returns to an equilibrium state. Nonetheless, when the system becomes unstable, the solution does not converge to an equilibrium point, but to a new equilibrium state defined by a limit cycle as in Figure 33 where the system leaves the equilibrium point and jumps to a limit cycle in which the amplitude of the oscillation remains constant for the rest of the time span of the simulation. In the next numerical integrations, we show the behavior of the system when we increase the feedback gain of the reactor $\kappa>1$ for three different values of the feedback gain $\kappa=[1.45,1.55,1.75]$, as illustrated in Figures 34-36.

In Figure 34 the amplitude of the oscillation (equal to the maximum value of the pulses i.e., the signal envelope) follows a smooth curve and promptly converges to the final amplitude. For $\kappa=1.55$ (Figure 35) the oscillation is following a new limit cycle of its own with twice the original period. This causes the signal to periodically exhibit two peaks of different magnitude and in the last case where $\kappa=1.75$ (Figure 36) the oscillation exhibits several pulses of different magnitude; the system in this case never converges to a final amplitude. In essence the amplitude of the limit cycle has become unstable and is following a new limit cycle of its own with $2^{n}$ the original period. This process is known in the literature by the name of period doubling pitchfork bifurcation. Such process continues as the value of $\kappa$ is increased further. The amplitude of each new limit cycle becomes unstable at critical values of the feedback gain, $\kappa_{j}$ and a new limit cycle of twice the original period appears. This process is visualized in the Orbit diagram (or Route to chaos) shown in Figure 37.
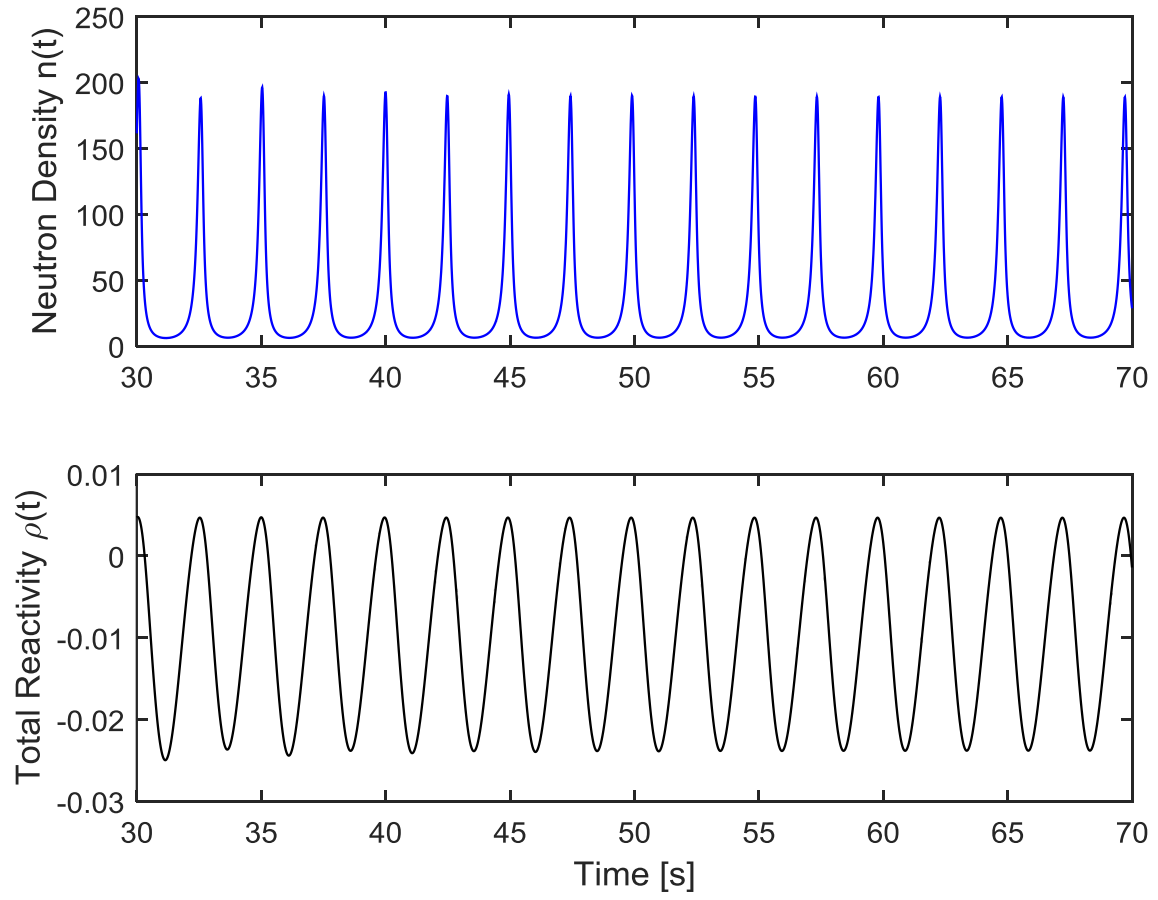
Figure 34. Unstable ROM output for $\kappa=1.45$.
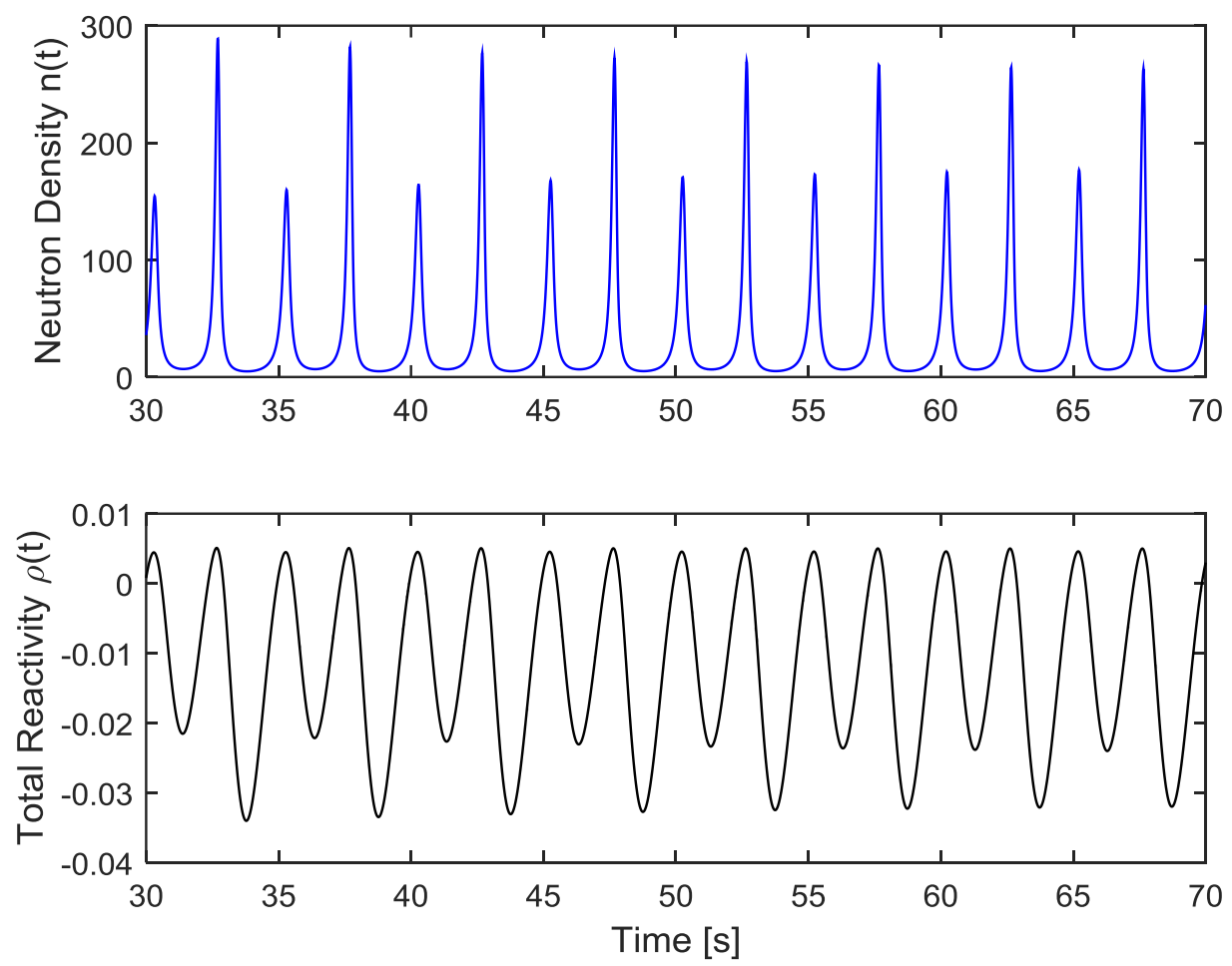

Figure 35. Unstable ROM output for $\kappa=1.55$.
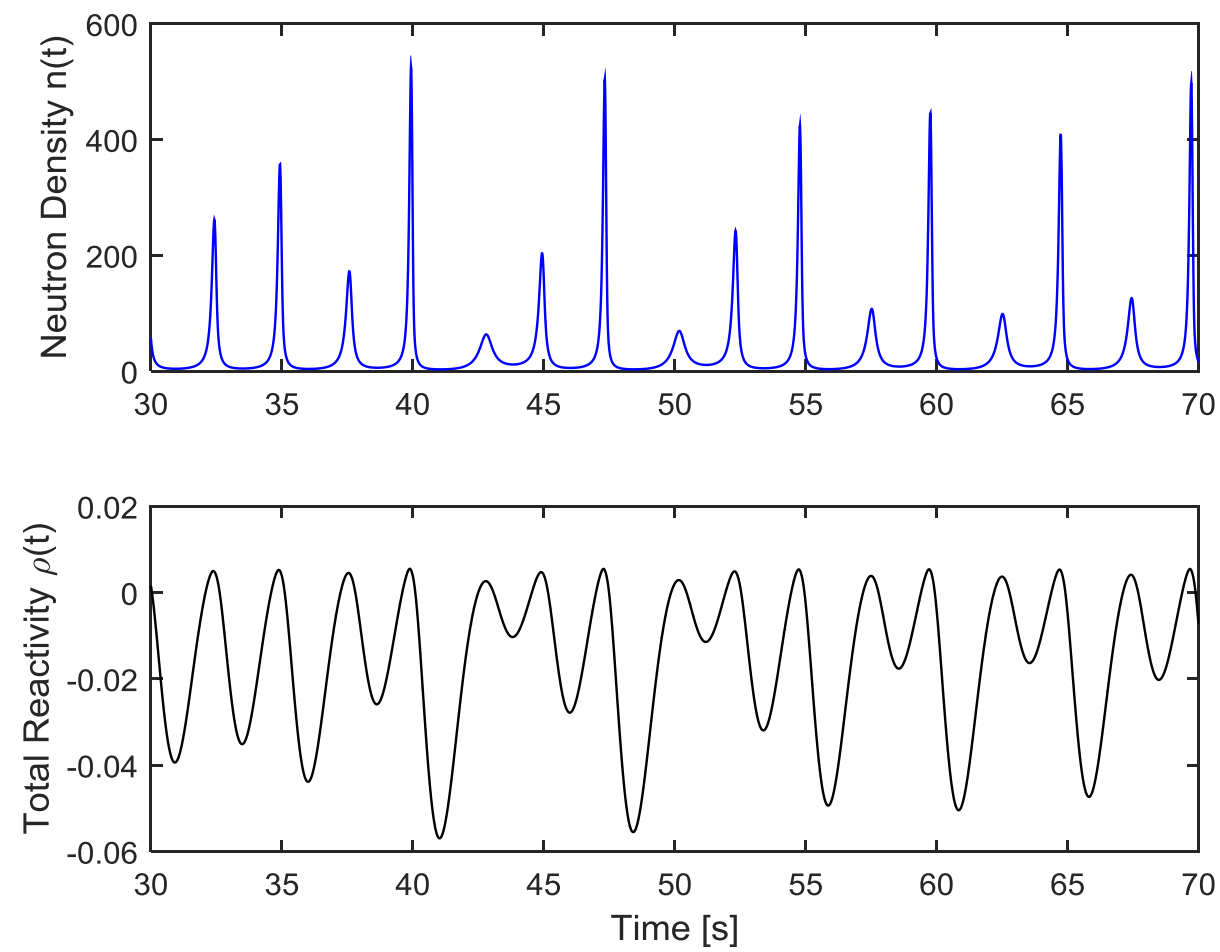
Figure 36. Unstable ROM output for $\kappa=1.75$.
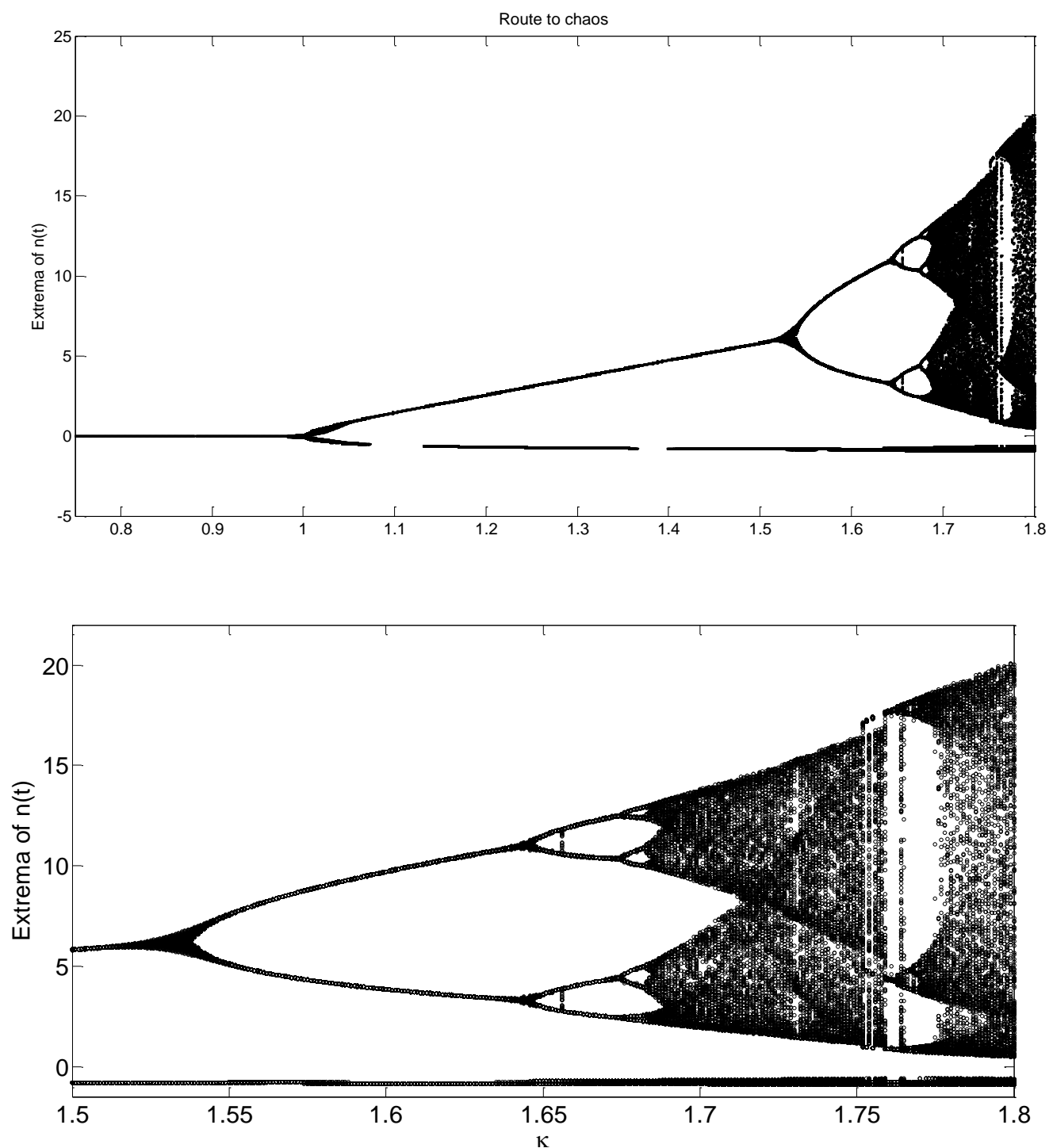

Figure 37. Route to chaos showing the accumulation of critical bifurcation values and the onset of aperiodicity.

In the diagram shown in Figure 37. The maxima of the oscillation are plotted for several values of the feedback gain $\kappa$. In the region where $\kappa<1$ the model is stable and the maxima coincide with the equilibrium point. For a feedback gain between $\kappa \in(1,1.55]$ the equilibrium point is unstable but a new limit cycle exists with the amplitude defined by the maxima and minima of the oscillation. For a gain $\kappa>1.65$ this two turns limit cycle becomes unstable and a new four-turn limit cycle appears. In essence at every critical value, $\kappa_{j}$, each of the branches in the diagram split (bifurcates) into two new branches. This implies that the critical value $\kappa_{j}$ converge to an accumulation point $\kappa_{\infty}\left(\kappa_{\infty} \approx 1.71\right)$. The 
solution of the system for a feedback gain greater than $\kappa_{\infty}$ will be aperiodic or chaotic. Currently, no definition of the term chaos is universally accepted yet, but almost everyone agrees on three ingredients used in the following working definition: Chaos is aperiodic long-term behavior in a deterministic system that exhibits sensitive dependence on initial conditions (Strogatz, 2014). In such region, the solution converges to a close curve or to an equilibrium point. Such type of solution is called a strange attractor. This region in phase space attracts the trajectories towards it, but once inside, the trajectories repel each other, so that there is not a final closed curve to maintain equilibrium. A situation similar to this has been postulated to explain turbulence phenomena. In resume, according to this simple ROM that contains the most important features of a BWR system. The BWR is a complex dynamical system that exhibits period doubling pitchfork bifurcations when the feedback gain of the system is increased beyond the critical feedback gain value $\kappa=1$. If this gain keeps increasing beyond the accumulation point $\kappa_{\infty}$, the solution of the system will become aperiodic or chaotic.

\subsection{Decay Ratio estimation: Methodology and results}

In this section, numerical experiments are performed on artificial signals produced with the Reduced Order Model (ROM) previously introduced. In here, we estimate the decay ratio (DR) using a classical AR model. Our goal is to show the DR limitations to infer stability information of a complex dynamical system such as a BWR. The procedure to estimate the DR via AR modeling includes the next steps:

Method 7 DR estimation via AR modeling

Step 1. The BWR signal of interest (emulating a LPRM recording) $n(t)$ is preprocessed to remove its trend.

Step 2. The resulting signal of Step 1 is model through an autoregressive model. The Akaike information criteria (Akaike, 1974) is used to estimate the best possible AR model ( or apparently the best AR model) to describe the signal of interest.

Step 3. From the best possible AR model, the impulse response $h(t)$ of the system (of the reactor) is computed.

Step 4. The DR is estimated from the autocorrelation function of the impulse response $h(t)$, by computing the DR as the ratio between the second peak and the first peak of the ACF. 
The DR was estimated from a set of 300 BWR signals $n(t)$ for feedback gain values in the interval $\kappa \in[0.75,1.80]$, covering in this way the entire stable and unstable regions of the chosen ROM model, in order to observe the DR behavior of this chosen ROM (we wish to figure out whether the computed DR is able to follow the route to chaos plotted in Figure 37). Results of this DR estimation method are shown in Figure 38.
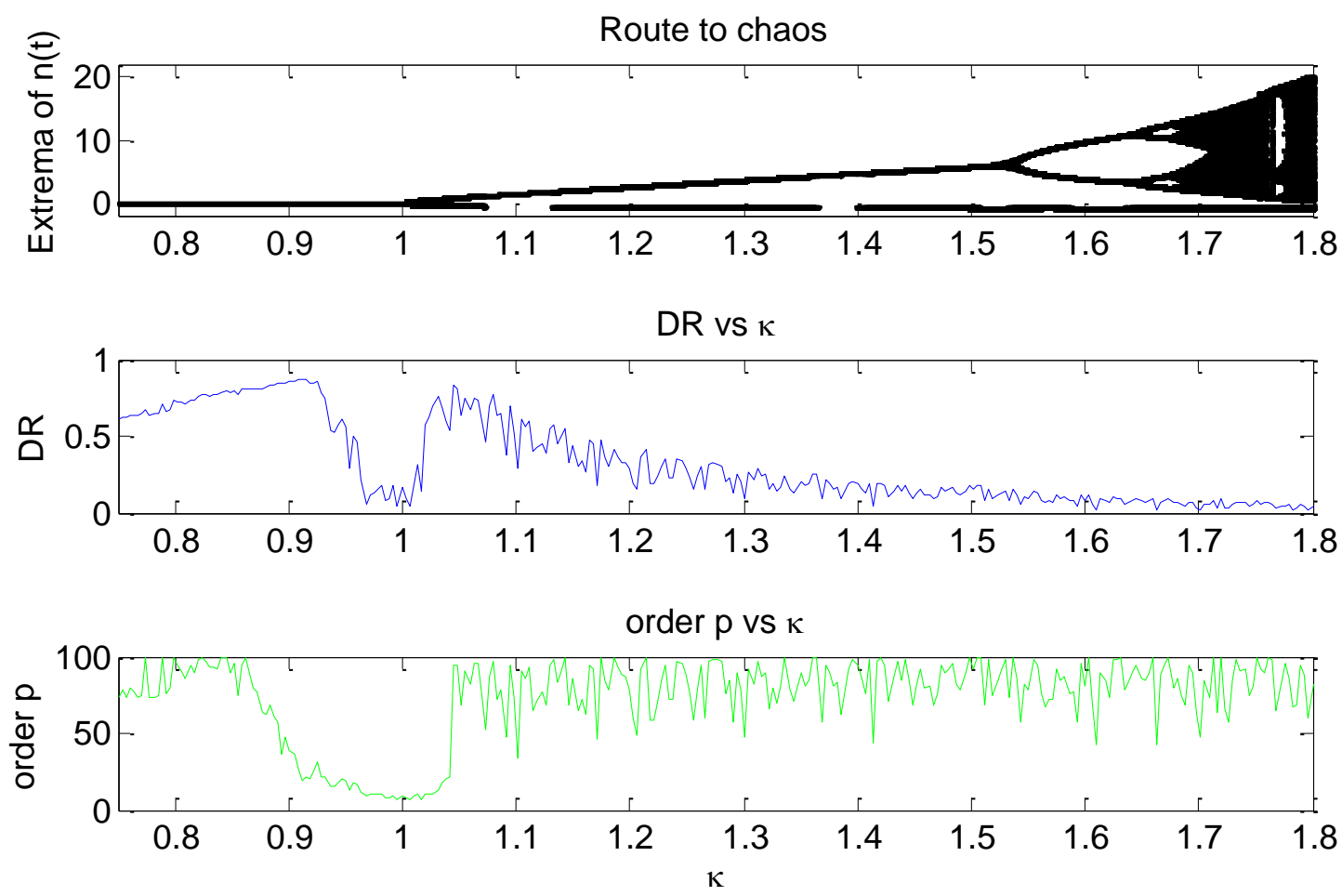

Figure 38. DR estimation for a set of signals with feedback gain values in the interval $\kappa \in[0.75,1.8]$.

The time span of the signals of interest is $130 \mathrm{~s}$, however, we are suppressing the first $30 \mathrm{~s}$ of the signal (to eliminate transients). The initial condition vector for the entire set of signals is kept at $[0.1,0.1,1,0,0]$, the numerical method is the standard Runge-Kutta 4th order method and the integration step is again $\Delta t=0.01 \mathrm{~s}$.

As we can see in Figure 38 (blue line), as soon as the feedback gain $\kappa$ increases beyond 0.75 , the DR increases accordingly, however, the DR is underestimated when $\kappa$ approaches the critical value $\kappa=1$ that destabilizes the BWR, the DR increases again until $\kappa$ approaches the feedback gain value $\kappa=1.1$ already within the unstable region. From this value and onwards, the DR is again underestimated and does not follow adequately the route to chaos of this ROM, which is an indication of the lack of accuracy and the lack of 
predictability the DR possesses to assess the instability of a complex dynamical system such as a BWR.

Besides, the order $\mathrm{p}$ (green line) of the optimal AR model is too high to model unstable ROM signals. These high $\mathrm{p}$ order values in conjunction with poor DR values that did not get the proper BWR information about its instability (i.e., dynamics) are a proof that AR models at the core of BWR stability techniques are not the best option to study BWR stability (the same remark applies to the linear DR indicator, which does not capture the complex BWR dynamics shown by this ROM): Besides, methods using the correlation function, the impulse response (obtained by AR models) and neural networks were compared by Van der Hagen, 1995 and many drawbacks and inaccuracies of such methods are indicated in that reference in detail. Overall, Van der Hagen, 1995 calls for a reconsideration of the practical use of the DR. Therefore, the BWR stability problem needs to be readdressed in a different manner with tools that accommodate as much as possible, the non-linear dynamics of a BWR to infer instability (in particular, such tools must be able to detect the beginning of an instability event). In the next chapter, the Shannon Entropy (SE, Shannon, 1948) is studied to build a methodology to assess the complexity of BWR signals to infer information about BWR instability.

\subsection{Chapter 4 Conclusions}

The selected reduced order model (ROM) is simple and yet, adequate enough to highlight that BWR systems under density wave instability exhibit a chaotic nature described by period doubling pitchfork bifurcations. The density wave instability is introduced in the differential equation model via the parameter $\kappa$, when it exceeds the ROM stability threshold of $\kappa=1$. Highly chaotic behavior for large $\kappa(\kappa>1.8)$ is characterized by strange attractors. The BWR does not behave as a second order dynamical system (i.e., a harmonic oscillator). Therefore a simple linear parameter such as the DR will not accommodate altogether the complex nature of BWR under instability. The DR is in our estimate an unreliable BWR instability indicator. According to our experiments with a classic AR-DR model methodology, we conclude that the DR measurement misses the bifurcation phenomena which occurs in unstable BWR systems. The DR only predicts linear stability characteristics (stable BWR behavior). Thus, it is desirable to study more sophisticated stability indicators that do take into account the non-linear and non-stationary behavior of BWR recorded signals with the ambitious objective of enhancing BWR security systems and protocols. In the following chapters we will introduce the studied nonlinear instability indicators to address the appraisal of BWR instability phenomena. 


\section{Chapter 5}

\section{BWR stability indicator 1: The Shannon Entropy}

In this chapter, we explore the Shannon Entropy (SE) as a possible non-linear stability indicator for BWRs. The Shannon Entropy is a concept introduced by Claude E. Shannon (Shannon, 1948) to characterize a discrete source through the content of the information of this source. In other words, the SE is a statistical index that quantifies the complexity of a signal. In our case, the BWR instability problem is studied by quantifying the intricacy of BWR signals through this proposed indicator. A low SE estimation indicates a predictable BWR event (a stable event) whereas a high SE value indicates an unpredictable BWR event (an unstable event). The SE estimation was validated with artificial signals issued from the ROM studied in the previous chapter and with real BWR signals that stem from the Forsmark (Verdú et al., 2001) and Ringhals (Lefvert, 1996) stability benchmarks and from a Laguna Verde instability event that happened in the year 1995.

\subsection{The Shannon Entropy}

In order to capture the complex dynamics of a BWR system, the Shannon Entropy (SE) is studied. In statistical mechanics and information theory, entropy is a functional that quantifies the information content of a statistical ensemble or equvalently, the uncertainty of a random variable. Its application in various scientific disciplines is countless. Nonetheless, the most important example of such a functional is the Shannon Entropy (also known as average information), the concept is established as: Consider a discrete random variable $c$, which can take a finite number of $M$ possible values $c_{i} \in\left\{\mathrm{c}_{i}, \ldots, \mathrm{c}_{M}\right\}$ with corresponding probabilities $p_{i} \in\left\{\mathrm{p}_{1}, \ldots, \mathrm{p}_{M}\right\}$. Its entropy $H_{S}(\mathrm{c})$ is defined as:

$$
H_{S}(c)=-\sum_{i=1}^{M} p_{i} \ln \left(\mathrm{p}_{i}\right)
$$

In general, the probability distribution for a given stochastic process is not known, and, in most situations, only small data sets from which to infer the entropy are available. For instance, it could be of interest to figure out the Shannon Entropy of a given BWR signal. In such circumstances, one could estimate the probability of each element $i$ to occur, $p_{i}$, by making some assumptions on the probability distribution, as for example:

i. Parametrizing it.

ii. Dropping the most unlikely values.

iii. Assuming some a priori shape for the probability distribution. 
Nevertheless, the easiest and most straightforward path to estimate them is by counting how often the value $c_{i}$ appears in the available data set. Denoting this number by $l_{i}$ and dividing by the total size $N$ of available data set, we can obtain a relative frequency estimator given by:

$$
\hat{p}_{i}=\frac{l_{i}}{N}
$$

Which naively approximates the probability $p_{i}$ linked to the value $c_{i}$. With this simple estimator in mind, the easiest way to compute the SE of the data set can be done by simply replacing the probabilities $p_{i}$ by $\hat{p}_{i}$ in the entropy functional, giving an estimate of the Shannon Entropy:

$$
H_{s}(c) \approx H_{s}^{\text {naive }}(c)=-\sum_{i=1}^{M} \hat{p}_{i} \ln \left(\hat{p}_{i}\right)=-\sum_{i=1}^{M} \frac{l_{i}}{N} \ln \left(\frac{l_{i}}{N}\right)
$$

The quantity $H_{s}^{\text {naive }}(c)$ is an example of an entropy estimator proposed by (Knuth, 2006), in a very similar sense as $\hat{p}_{i}$ is an estimator of $p_{i}$. In particular the minimum $H_{s}(c)=0$ is reached for a constant random variable, i.e., a variable with a determined outcome, which reflects in a fully localized probability distribution $p_{i}=1$ and $p_{i}=0$ for $i \neq j$. At the opposite, $H_{s}(c)$ is maximal, equal to $\ln (M)$, for a uniform distribution $\left(p_{1}=p_{2}=\ldots=p_{M}\right.$ ) . The SE is a quantity that increases with the number of possible states: for an unbiased coin, $H_{s}(c)=\ln (2) \approx 0.6931$ while for an unbiased dice $H_{s}(c)=\ln (6) \approx 1.7918$. To estimate Equation (21), a histogram is required to infer the probabilities $p_{i}$ of the data set. In this work, the number of bins $M$ of such histogram was computed with an optimal estimator proposed also in Knuth, 2006. Shannon initially proposed this functional to quantify the information loss in transmitting a given message in a communication channel. A noticeable aspect of Shannon approach is to ignore semantics and focus on the pyhsical and statistical constraints limiting the transmission of a message, regardless of its meaning. The source generating the inputs $c_{i} \in c$ is characterized by the probability distribution $p_{i}$. Shannon Entropy $H_{s}(c)$ thus appears as the average missing information. That is, the average information required to specify the outcome $x$ when the receiver knows the distribution $p_{i}$. It equivalently measures the amount of uncertainty represented by a probability distribution. In the context of communication theory, it amounts to the minimal number of bits that should be transmitted to specify $x$. Based on these facts and considering that the estimator in Equation (21) is the easiest way to estimate SE, it is the estimator used in our proposed methodologies to study the BWR instability due to density waves. The SE, estimated by our naive estimator, quantifies the uncertainty of the artificial studied signals. Through this approach, the instability problem of a chaotic dynamical system such as a BWR is studied. The SE is our tool to study reactor instability and as 
such, the SE might serve as an alternative option to the conventional DR indicator. Our goal is to detect through SE the beginning of an incipient instability event (via a stability monitor), prior any further development of that emerging unstable event. We are also interested in gaining from the SE indicator as much information as possible regarding the dynamics of a BWR system.

\subsection{BWR stability monitor based on the Shannon Entropy for artificial signals}

In this section, a Shannon Entropy stability methodology is introduced, this technique is rooted in the SE estimator given before in Equation (21) and in the improved complete ensemble empirical mode decomposition method. The SE / improved CEEMDAN stability methodology is given by the following steps:

1. The considered neutronic signal $n(t)$ of interest obtained from the BWR ROM model (March-Leuba, 1986) discussed in Chapter 4 is decomposed using the improved CEEMDAN method for an ensemble of $I=100$ realizations of white noise of standard deviation $\varepsilon_{O}=0.2$, obtaining in this way the corresponding IMFs.

The selection of the chosen improved CEEMDAN indicators $I$ and $\varepsilon_{o}$ were taken from Colominas et al., 2014. It is worth mentioning that this information is contained in the residue of this decomposition.

2. The Hibert-Huang transform (HHT) is computed in order to get the instantaneous frequencies contained in each IMF.

3. When tracking these frequencies, it is possible to get the mode linked to instability processes (IF highly concentrated around $0.5 \mathrm{~Hz}$ ). In this regard, two possible ways may emerge:

- The original entry signal $n(t)$ is considered for further processing in cases where there is no IMF linked to instability (i.e., the BWR is within the stable region and no meaningful instantaneous frequencies around $0.5 \mathrm{~Hz}$ were found).

- The IMF, from the decomposition of $n(t)$ linked to BWR instability is considered for further processing when meaningful instantaneous frequencies around $0.5 \mathrm{~Hz}$ were found (i.e., the BWR is within the unstable region).

4. The Shannon Entropy of the tracked IMF (or the original entry signal $n(t)$ when the BWR is in a stable state) is computed considering the estimator given in Equation (21), using the probabilities estimator given by equation (20). The optimal number of bins $M$ for the histogram, is calculated with a technique given in Knuth, 2006 in the interval $5 \leq M \leq 20$.

5 . The mean and variance of the SE is calculated. 
6. In order to range the SE between 0 and 1, the following normalization is given (Liang et al., 2016):

$$
\hat{H}_{s}^{\text {naive }}(\mathrm{c})=\frac{-\sum_{i=1}^{M} \hat{p}_{i} \ln \left(\hat{p}_{i}\right)}{\ln (M)}
$$

Based on this methodology, the following experiments were implemented. We estimated global SE values with the estimator given previously by equation (21) and the normalized by the equation (22), in order to figure out whether the SE is able to follow accordingly the route to chaos of the chosen BWR ROM (shown previously in Figure 37). The initial condition vector for all the BWR ROM signals is kept again at $[0.1,0.1,1,0,0]$, the numerical integration method is the Runge-kutta 4th order method and the integration step size is $\Delta t=0.01 \mathrm{~s}$. Before to show the complete results sweeping this Route to chaos, we analyze two specific cases: one stable case and one unstable case to contrast what a low SE value means from a high one.

\subsubsection{Stable BWR signal}

Figure 39 shows the neutron density $n(t)$, obtained from the studied ROM considering a stable behavior of the BWR. The time span of the signal is $130 \mathrm{~s}$, however, the first $30 \mathrm{~s}$ of the signal are ignored (transient suppression). The value of the feedback gain $\kappa$ is 0.75 .

Figure 40 shows the first 5 IMFs of the improved CEEMDAN decomposition of the signal shown in Figure 39 from a total of 12 IMFs. The amplitude (and thus the energy) of the IMFs along time is not meaningful, thus our methodology detects these weak oscillations as an indication of a BWR stable operation (the IMF amplitude is in average $<10^{-3}$ ).

Figure 41 shows the instantaneous frequencies (IF) of the IMFs shown in Figure 40. No meaningful IF oscillation is highly concentrated around $0.5 \mathrm{~Hz}$, thus, the IFs are of little interest when the BWR is in a stable state, we thus calculate the SE of the original signal $n(t)$. The global estimated SE (normalized according to $\ln (\mathrm{M})$ in order to provide $\mathrm{SE}$ values in the interval $0 \leq S E \leq 1$ ) of this signal is $S E=0.1883$. This low $S E$ value indicates that the uncertainty (thus the signal in Figure 39 has a high predictability) of the studied signal is not so high, thus, the BWR is located in its stable region. The estimated number of bins $M$ for this signal is 6 (calculated by the Knuth, 2006 proposal). 


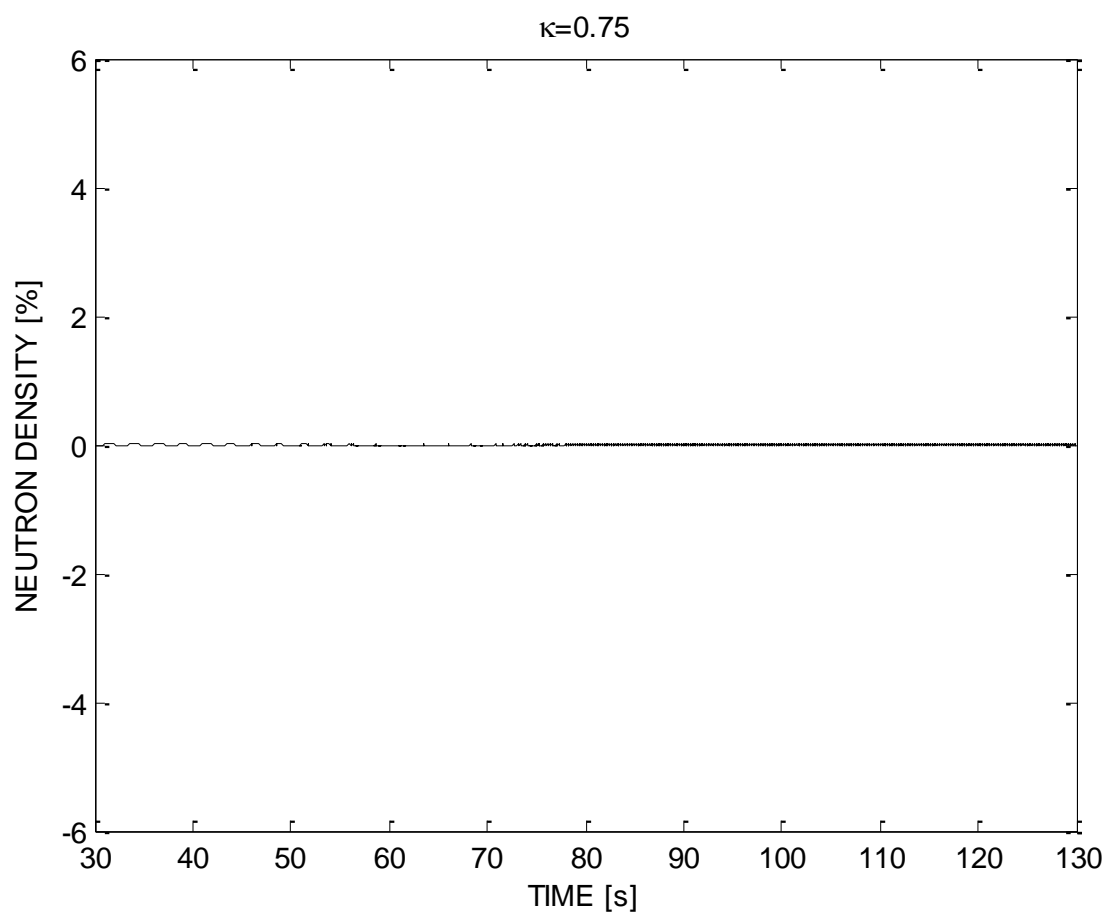

Figure 39. Stable BWR signal $n(t)$ of the feedback gain value $\kappa=0.75$.
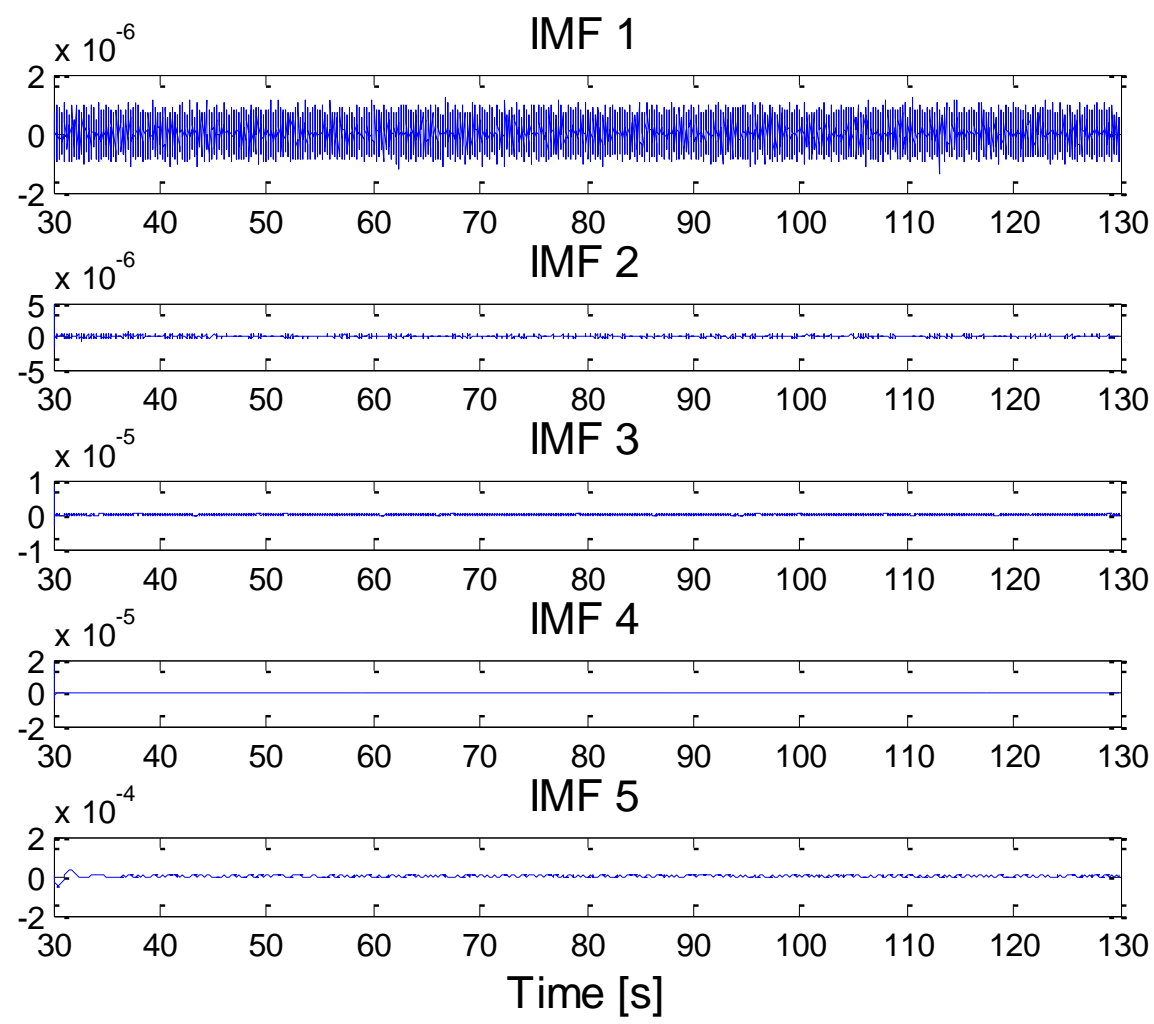

Figure 40. Improved CEEMDAN decomposition of the stable studied signal. 

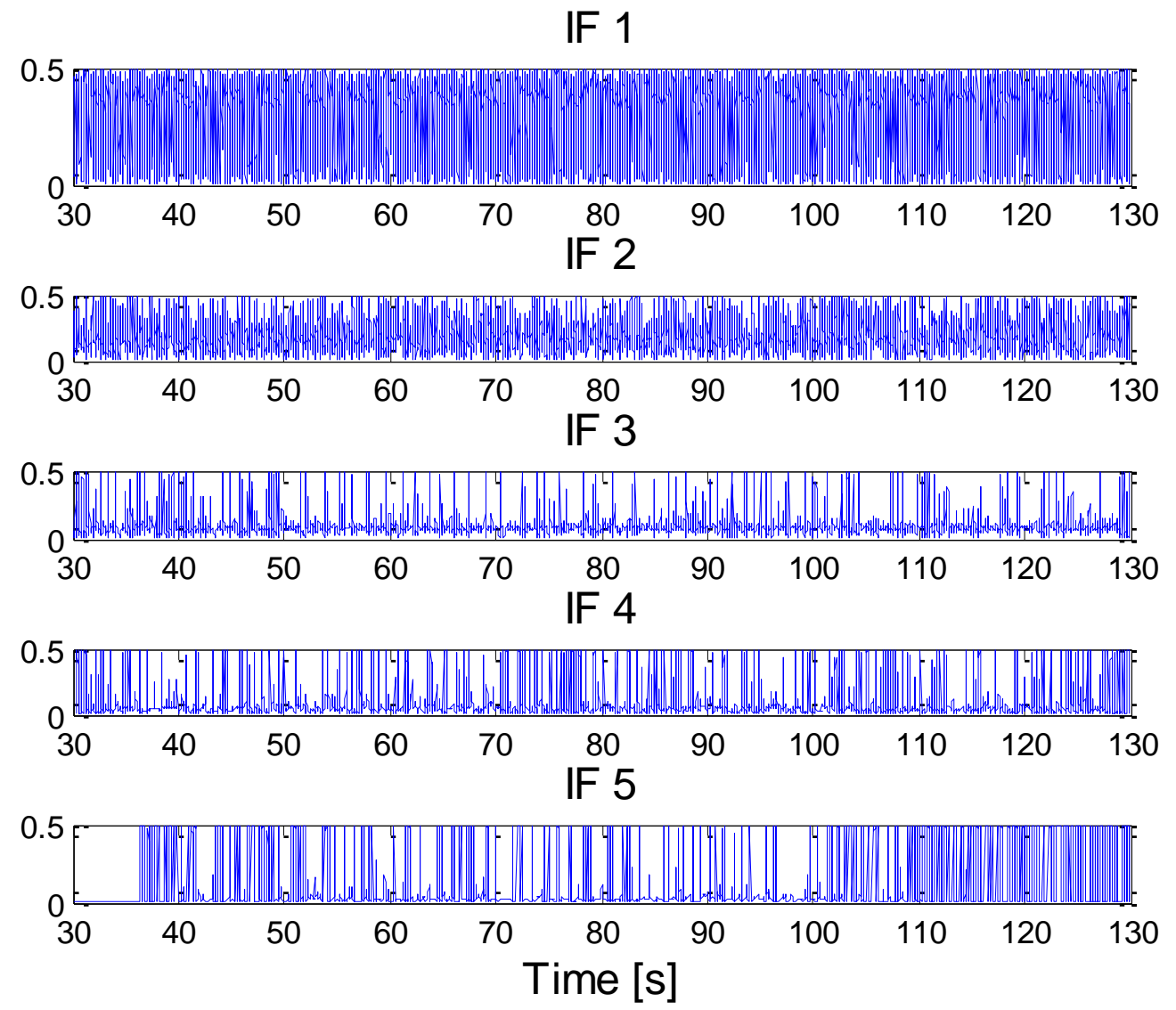

Figure 41. IFs of the IMFs (computed by means of HHT) shown in Figure 40. 


\subsubsection{Unstable BWR signal}

Now, the feedback gain of the ROM is increased beyond its critical value $k=1$ (border between stable and unstable regions). The BWR signal $n(t)$ is now unstable. This unstable signal is shown in Figure 42. The feedback gain value is now $\kappa=1.2$.

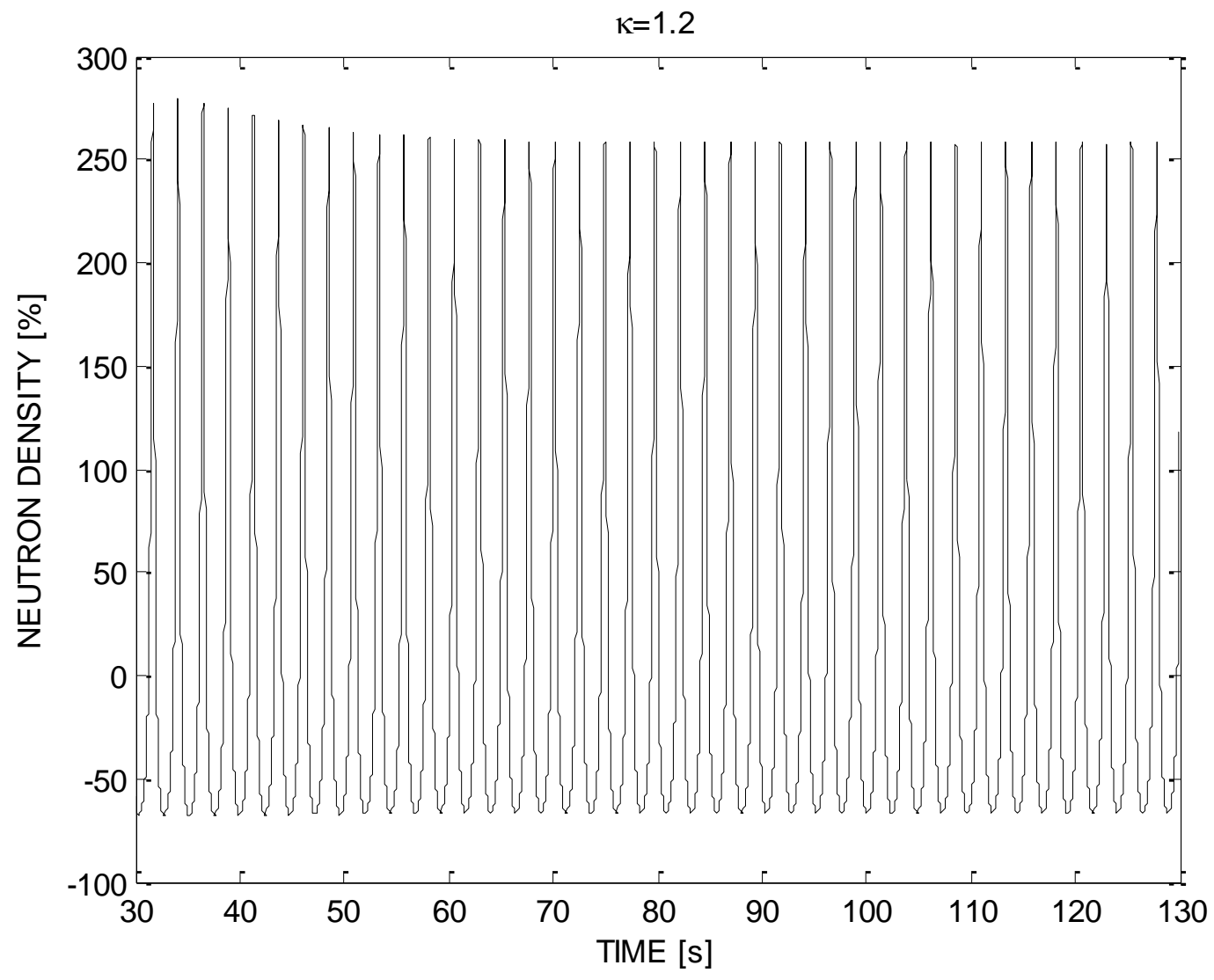

Figure 42. Unstable BWR signal $n(t)$ of feedback gain value $\kappa=1.2$.

Figure 43 depicts IMFs of the improved CEEMDAN decomposition of this unstable signal shown in Figure 42. Only, IMF 4 to IMF 8 are shown in Figure 43, because the IMF linked to BWR instability is the IMF 7 whose IF (i.e., IF 7 from the total IMF set, see Figure 44 to observe the respective IFs) is oscillating around $0.5 \mathrm{~Hz}$ (the energy of this IF 7 is highly concentrated around $0.5 \mathrm{~Hz}$ ). Thus, the IMF 7 is chosen for further processing. 
IMF 4
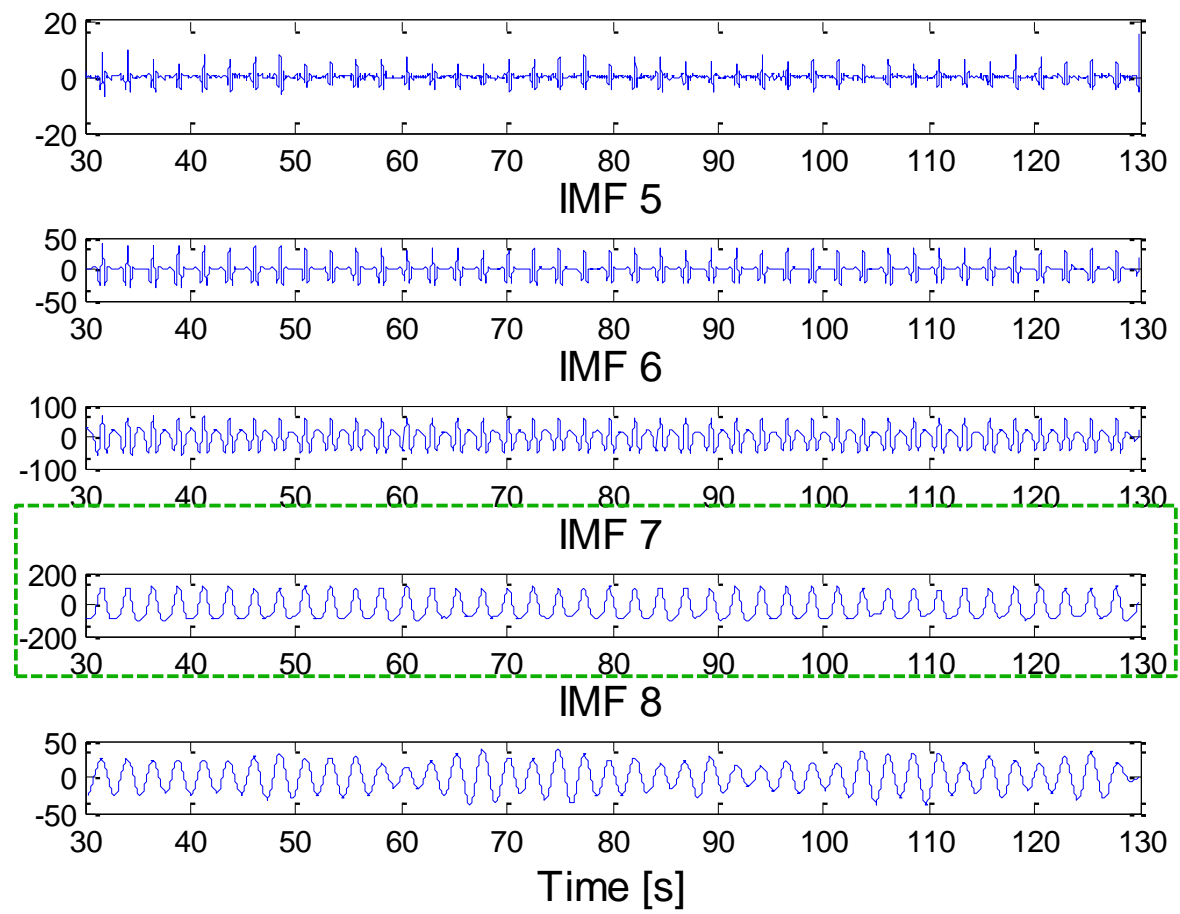

Figure 43. Improved CEEMDAN decomposition of the BWR signal shown in Figure 42.

IF 4
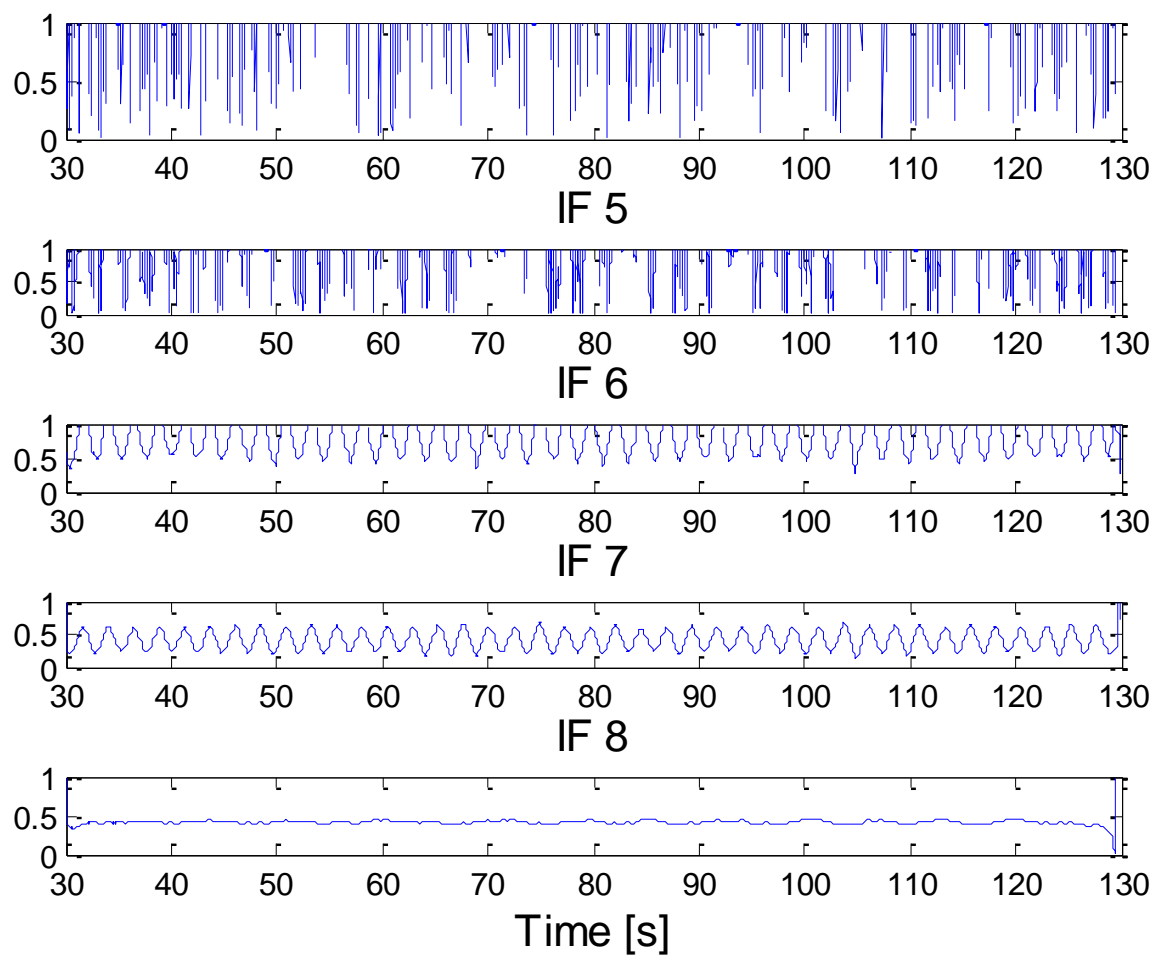

Figure 44. IF of the IMFs (computed by means of HHT) shown in Figure 43. 
The SE of the IMF 7 (the IMF linked to instability) is computed. The global estimated SE (normalized according to $\log (\mathrm{M})$ in order to provide $\mathrm{SE}$ values in the interval $0 \leq \mathrm{SE} \leq 1$ ) is $S E=0.8955$. This high $S E$ value indicates that the uncertainty (thus the signal in Figure 42 has low predictability) of the IMF 7 is high, thus the BWR is located in its unstable region. The estimated number of bins $M$ for this signal is 20 (provided by the method given by Knuth, 2006).

\subsubsection{Full SE sweep of the ROM Route to chaos}

The global SE was estimated from a set of 300 BWR signals $n(t)$ for feedback gain values in the interval $\kappa[0.75,1.80]$, to cover the entire stable and unstable regions of the studied ROM model to observe the behavior of the chosen SE estimator. Results of this experiment are shown in Figure 45. The time span of the signal of interest is $130 \mathrm{~s}$. However, we are suppressing again the first $30 \mathrm{~s}$ of each signal. The Runge-Kutta indicators are kept as before and each SE value is normalized according to its optimal estimated value $M$.
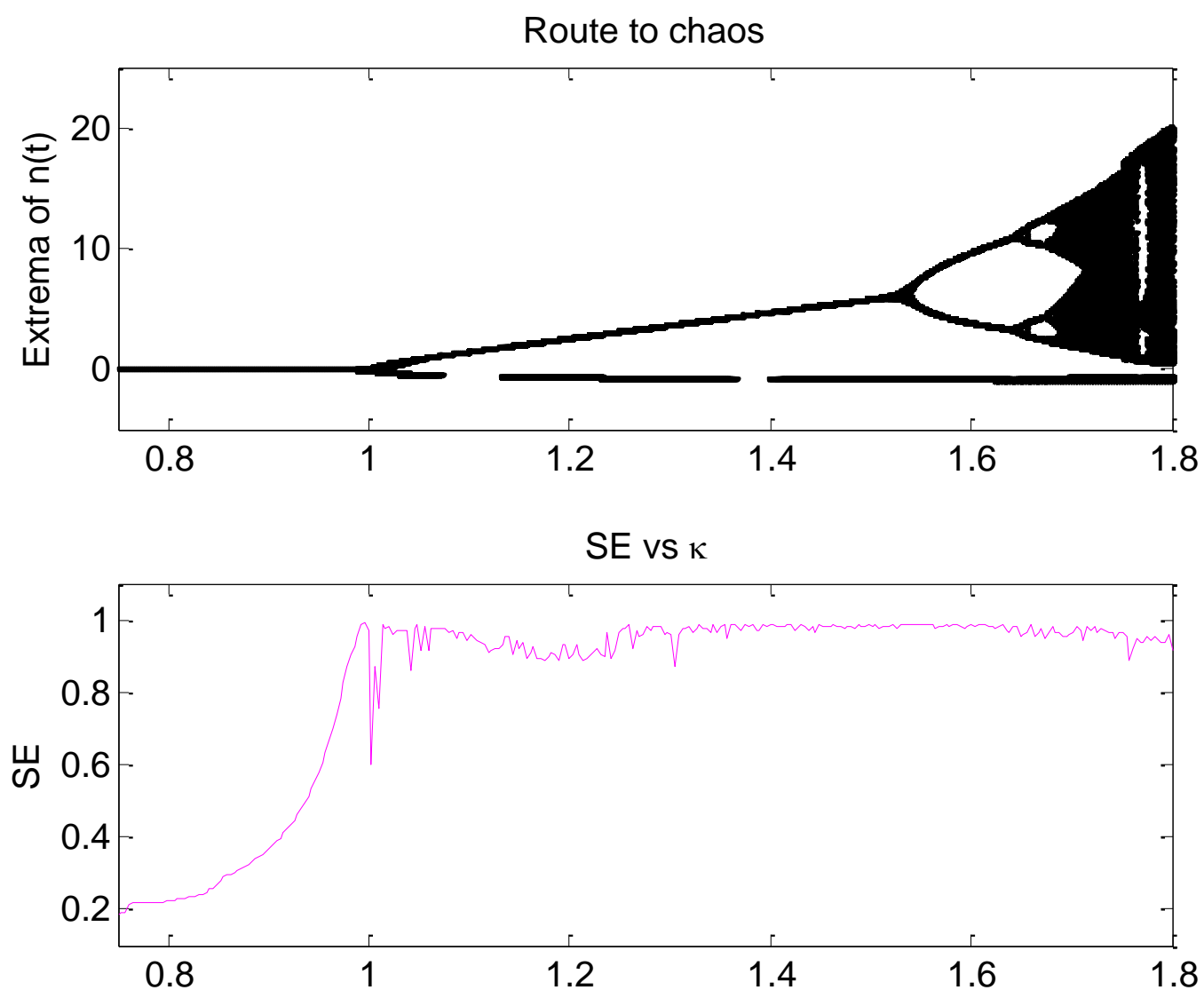

Figure 45. SE sweep of the ROM route to chaos. 
The SE sweep of the ROM route to chaos, clearly permits to separate between BWR stable states from BWR unstable states, the SE behavior in the stable region $(\kappa<1)$ increases as soon as the ROM feedback gain approaches the critical value which destabilizes the reactor $(\kappa=1)$. Low SE values indicate BWR stability (and thus predictability) whereas high ones indicate instability (high uncertainty) or that the system is being destabilized due to feedback gain increasing values. Beyond the critical value $(\kappa=1)$, the SE remains high throughout the entire unstable ROM region indicating instability.

Figure 46 shows the estimated optimal number of bins $M$ for each case, estimated with the Knuth, 2006 technique. In the stable ROM region $(\kappa<1)$, the optimal number of bins is 5 for all the stable cases (low BWR complexity). As soon as the feedback gain attains its critical destabilizing value $(\kappa=1)$, the optimal number of bins increases dramatically to almost 20 and in the unstable ROM region $(\kappa>1)$, the optimal number of bins increases dramatically to almost 20 and in the unstable ROM region $(\kappa>1)$ the value $M$ oscillates between 14 and 20 bins to represent the histogram of the improved CEEMDAN IMF linked to BWR instability. Thus, the unstable BWR IMF signals require an elevated bin number $M$ to provide optimal histograms to represent data.

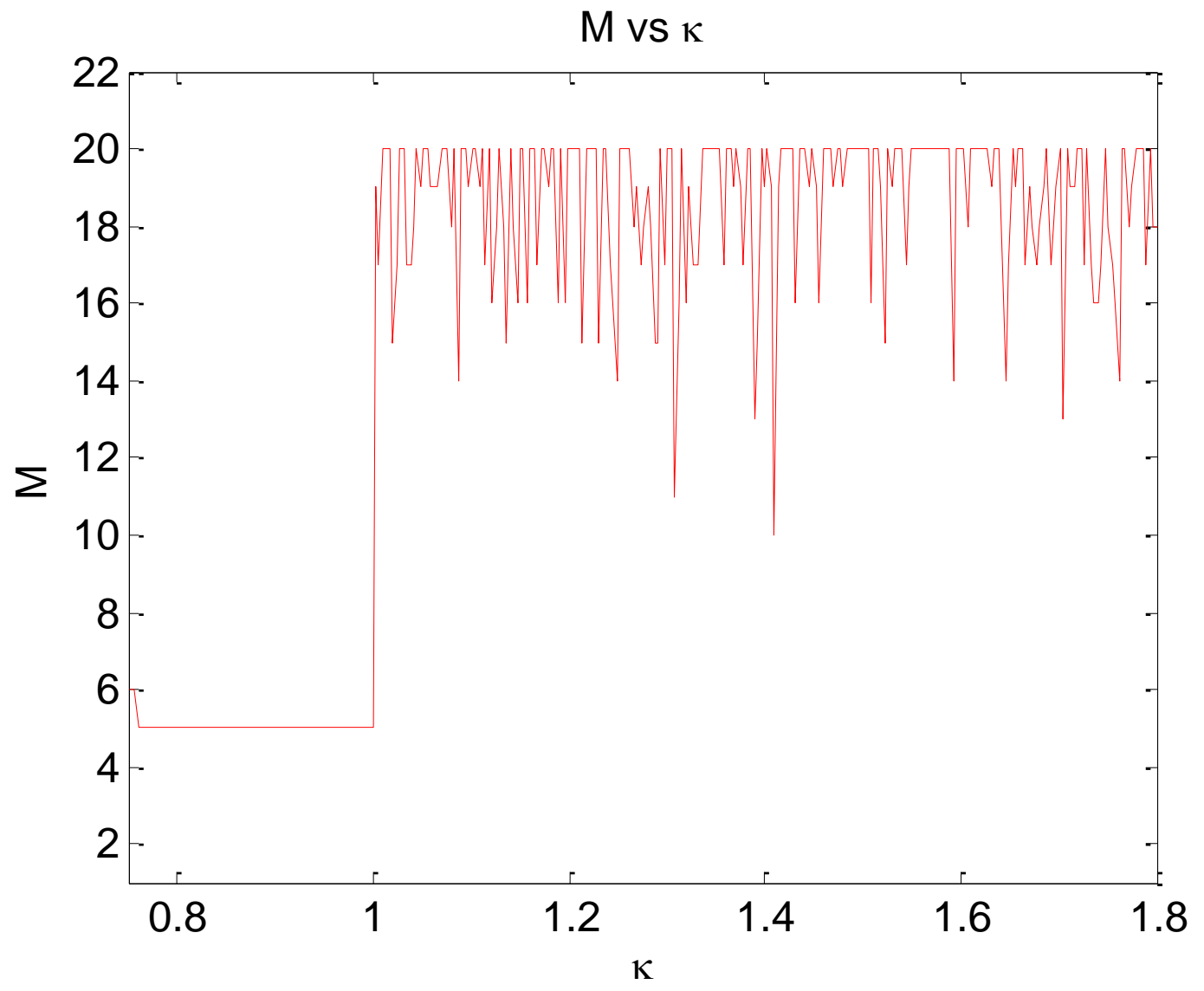

Figure 46. Estimated number of bins $M$ to compute the SE sweep of the ROM route to chaos. 


\subsection{Non-linear stability analysis of BWR real signals based on noise assisted empirical mode decomposition variants and the Shannon Entropy}

In this section, a novel methodology based on an adaptive Shannon Entropy estimator and on Noise assisted empirical mode decomposition variants is presented. This methodology was developed for real-time implementation of a stability monitor. This methodology was applied to a set of signals stemming from several nuclear power plants (NPPs, RingalsSweden, Forsmark-Sweden and Laguna Verde-Mexico) under commercial operating conditions, that experienced instability events in the past.

\subsubsection{Methodologies based on Shannon Entropy}

In this section, two stability methodologies are introduced, labeled as methodology 1 and methodology 2, based on improved CEEMDAN and NA-MEMD respectively, to study individual BWR unstable events and multivariate ones.

Methodology 1: Stability Monitor based on the improved CEEMDAN and the SE

Step 1. The considered signal (APRM or LPRM) obtained from the BWR is segmented in windows of 15 s of duration each.

Step 2. Each segmented signal (APRM or LPRM) is studied using the improved CEEMDAN method for a number of realizations of the ensemble $I=100$ and standard deviation of the assisted noise $\varepsilon_{o}=0.2$, described above, obtaining in this way, the corresponding IMFs. It is worth mentioning that the APRM or LPRM signals are not being processed before. For instance, to remove the signal trend, due that this information is contained in the residue of the decomposition.

Step 3. The Hilbert transform of each IMF is computed in order to get the instantaneous frequencies contained in each IMF (this step is known as Hilbert-Huang transform).

Step 4. When tracking these frequencies, it is possible to get the mode linked to density wave $(D W)$ instability.

Step 5. The SE of the tracked IMF (mode of interest linked to BWR instability) is computed considering the estimator given in equation (21). The optimal number of bins $M$ is again 
calculated by the Knuth, 2006 proposal (Several rules of thumb exist for determining the number of bins, such as the belief that between 5 - 20 bins is usually an adequate number).

Step 6. The mean and variance of the SE are calculated and averaged along all the studied segments of $15 \mathrm{~s}$.

Step 7. The SE estimates are ranged between 0 and 1 in the same way as in the previous section.

Methodology 2: Stability Monitor based on the NA-MEMD and the SE

Step 1. The considered multivariate signal (an array of $N$ independent LPRM signals) obtained from the BWR are segmented in small windows of $15 \mathrm{~s}$.

Step 2. These segments ( of 15 s each of time span) are decomposed in parallel through NA-MEMD in $N$ independent channels. Also, $m$ independent channels of white Gaussian noise are added (to mitigate the mode mixing issue) for decomposition ( $m=3$ for all of our computer experiments).

Step 3. After decomposition, discard the $m$ channels corresponding to the noise, giving a set of $N$-channel IMFs corresponding to the original signal segments.

Step 4. The Hilbert transform of each IMF is computed in order to get the instantaneous frequencies contained in each $N$-channel IMFs frequencies (i.e., the HHT).

Step 5. When tracking these frequencies, it is possible to get the IMF linked to density wave instability. In this regard, only the IMFs linked to DW instability are considered for further processing. Exploiting the NA-MEMD properties, the chosen IMFs of interest are all located at the same level of decomposition.

Step 6. The SE of the tracked IMFs (modes of interest linked to DW instability) are computed via Equation (21). The Knuth, 2006 proposal is used in a local way, within the interval $5 \leq M \leq 20$. There are thus, $N$ different values of $S E$ (each $S E$ value is linked to one LPRM in particular).

Step 7. The mean and variance of the SE values are calculated and averaged along all the studied multivariate segments of $15 \mathrm{~s}$.

Step 8. The SE estimates are ranged between 0 and 1 in the same way as in the previous section. 


\subsubsection{Results: methodologies performance and discussions}

The previous methodologies 1 and 2 are applied to BWR signals that stem from the Forsmark (Verdú et al., 2001), Ringhals (Lefvert, 1996) and the Laguna Verde Instability event (Gonzalez et al., 1995). The Ringhals plant stability benchmark test data has been widely applied to BWR stability studies because they cover various stability conditions, e.g., dominant fundamental mode related with in-phase instabilities, dominant first harmonic mode related with out-of-phase instabilities, and an overlapping of the two modes. The stability tests were performed (and controlled) in the Swedish BWR Ringhals Unit 1 from cycle 14 through cycle 17. The Forsmark benchmark is based on data from several measurements performed (performed) in the Swedish Reactor Forsmark 1 and 2, in the period 1989 to 1997 . The Laguna Verde instability event was recorded during an unstable event that occurred in 1995 and is considered in the literature as a prototype of an in-phase instability.

\section{Stability analysis of the chosen real cases through methodology 1}

This methodology 1 is applied to the next three following cases:

I. Case 4 of the Forsmark stability benchmark. This event is considered a challenging case to be analyzed due to the complexity of the phenomenon. For reasons of space, only this challenging case is presented in a detailed way. The studied Case 4 contains a mixture between a global oscillation mode and a regional (half core) oscillation. This event corresponds to a situation where the neutronic power reactor suffers abnormal and apparently unstable oscillations. This event corresponds to a situation where the neutronic power reactors suffers abnormal and apparently unstable oscillations. The C4_APRM and C4_LPRM_x signals correspond to average power range monitor (APRM) and local power range monitor (LPRM) registers respectively, during the instability event. The entire case 4 was studied (a total of 23 signals, 22 LPRMs plus 1 APRM). However, only the analysis of one signal (C4_APRM_1) is described in this work and the other results (22 LPRMs) are summarized in a table.

II. Case 9 cycle 14 of the Ringhals stability benchmark. Data given comes from measurements in the Swedish BWR reactor Ringhals 1. This case consists of a total of 36 LPRMs. Again, the whole case 9 (36 LPRMs) was studied, however, only the analysis of one signal (LPRM 1) is detailed in this work and the other results of LPRMs are summarized in a table.

III. An APRM signal that stems from the Laguna Verde BWR that was recorded during an unstable event that happened in 1995. On 24 January 1995 a power instability event happened in the Laguna Verde Unit 1, which is a BWR-5 and is operated since 1990 at a rated power of $1931 \mathrm{MWt}$. The instability event happened during a cycle 4 power ascension without fuel damage. When the thermal power reached $37 \%$ of the rated power, the recirculation pumps were running at low speed driving $37.8 \%$ of the total core flow. The flow control valves were set to their minimum, closed position in order to operate the recirculation pumps at a high speed. The 
drop in drive flow resulted in a core flow reduction of $32 \%$ and, a power reduction also of $32 \%$. Two control rods were also partially withdrawn during valve closure. The new low flow operating conditions resulted in growing power oscillations. This prototype of in-phase instability has been widely studied (Blazquez and Ruiz, 2003; Moreno, 2016).

\section{APRM signal from the Forsmark benchmark}

The studied signal in this subsection is the APRM 1 of the Forsmark stability benchmark, Case 4. This signal of interest is shown in Figure 47.

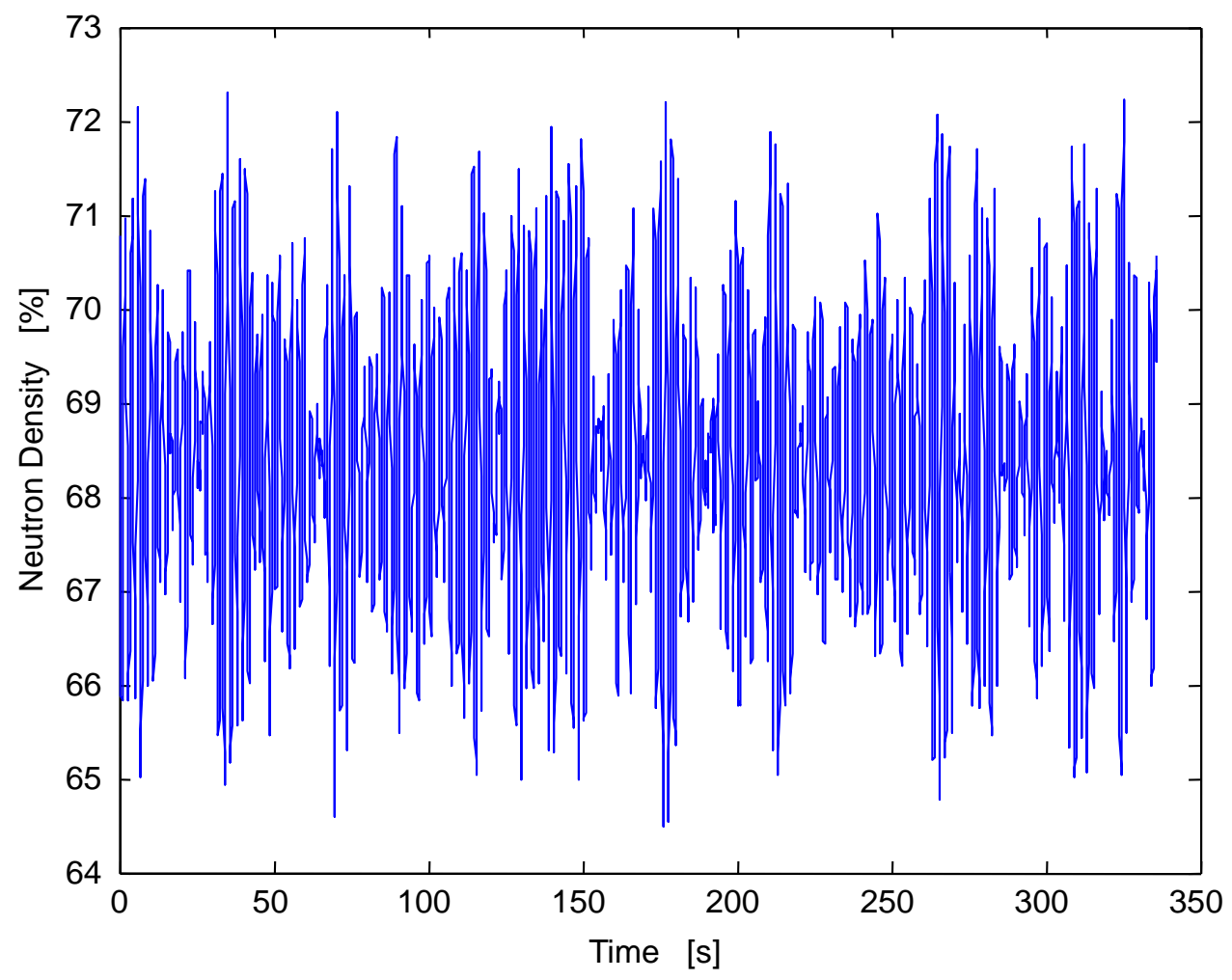

Figure 47. APRM 1 signal from the Forsmark stability benchmark. Case 4.

The Methodology 1 based on the improved CEEMDAN and the SE is applied to this signal. Such methodology splits the signal of interest in segments of $15 \mathrm{~s}$, later the segment is decomposed through improved CEEMDAN into IMFs, the HHT is calculated to obtain the instantaneous frequencies of the IMFs. The IF of interest linked to instability is tracked (the energy of this mode connected to BWR instability is highly concentrated around $0.5 \mathrm{~Hz}$ in the Fourier domain, according to previous BWR stability observations). Later, the IMF linked to the IF of interest is selected for SE calculation. Figure 48, shows the analysis of one studied segment that was decomposed through improved CEEMDAN into multiple 
IMFs and the IMF 3 is selected for more processing (because the IF (IF 3) of this IMF (IMF 3) is linked to BWR density wave instability, this key IF is shown in Figure 49).

Figure 50 shows a power spectral density estimation of the extracted IMFs of the studied segment, to visualize the spectrum of the IMF 3 linked to instability and to observe the improved CEEMDAN capabilities to compensate for mode mixing, which translates into less overlap of contiguous IMFs spectrums. Figure 51 shows the plot of the estimated SE of all of the studied segments of the signal of interest. Also, in this same figure, a DR estimate of the segments is shown to illustrate the performance of the SE over the DR to analyze the stability of the studied signal. The DR was estimated in the same way as in (OlveraGuerrero et al., 2017). We have established empirical stability thresholds based on our numerical experiments for the SE (Although more experiments are needed in this direction to accurately confirm this finding, bu such studies leave the scope of this work). This stability threshold value is located around 0.8 (a stable segment has a SE $<0.8$ whereas an unstable one has a $\mathrm{SE}>0.8$ ). Now, regarding the $\mathrm{DR}$, a stable segment has a $\mathrm{DR}<1$. For this signal, the DR estimate indicates the beginning of an unstable event (an incipient one) whereas the SE throughout the whole time span of the signal, points to the existence of a fully developed instability event from the very beginning of the simulation. Figure 52 shows the estimated number of bins $M$ for the extracted IMF for the studied case which remained very close to 5 bins and jumping beyond 5 in some segments.
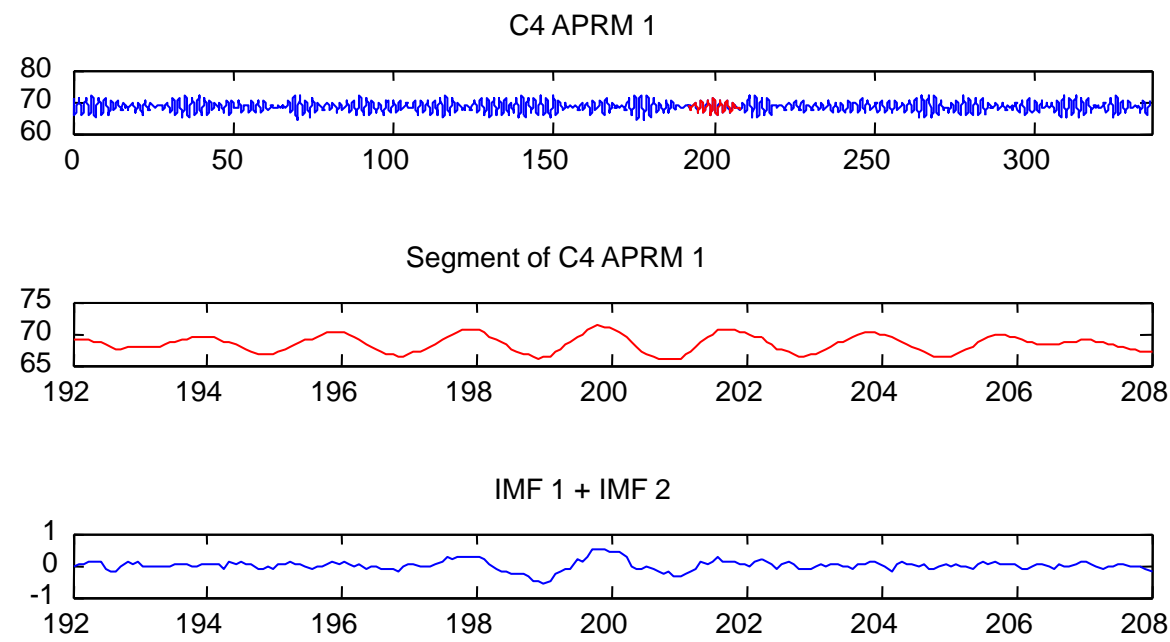

IMF 3

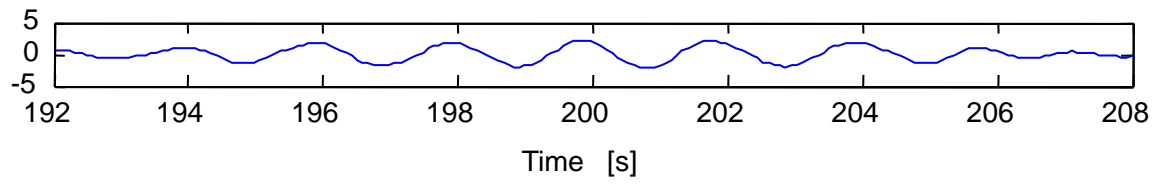

Figure 48. Improved CEEMDAN decomposition of one of the segments of the APRM 1. 
Segment of C4 APRM 1

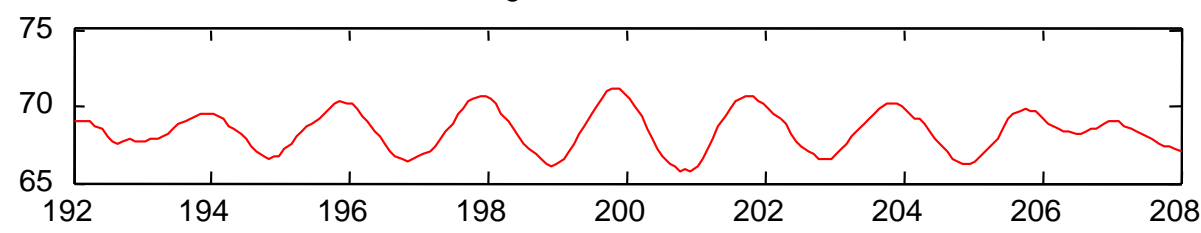

IMF 3 (linked to BWR instability)

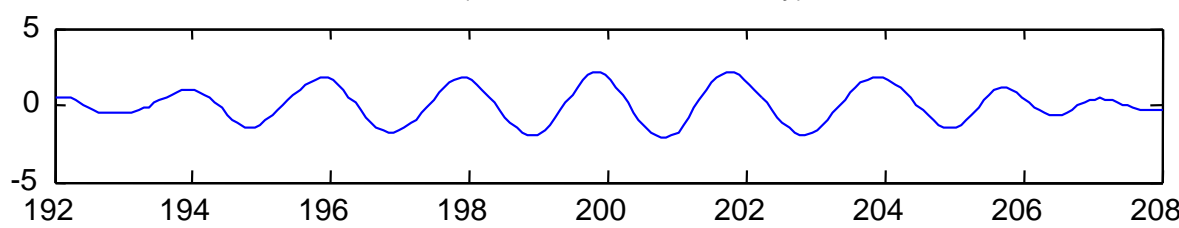

IF - IMF 3

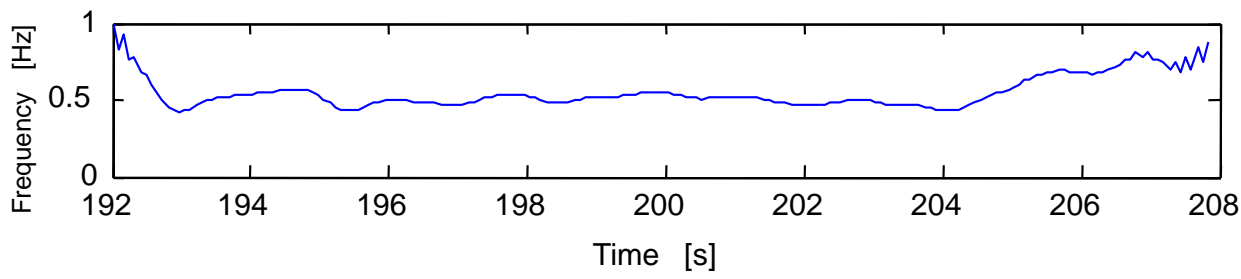

Figure 49. IF 3 linked to BWR density wave instability.

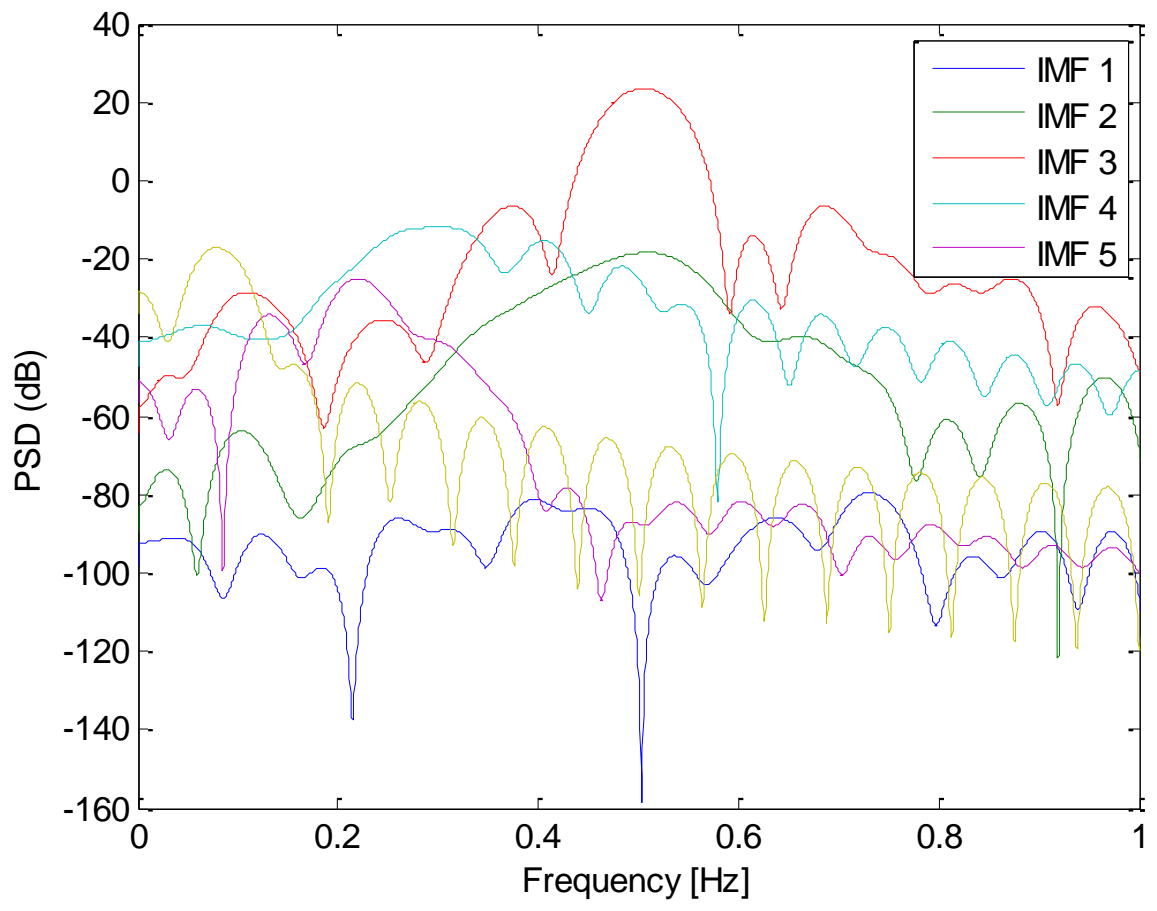

Figure 50. PSD estimate of the extracted IMFs of the studied segment. 


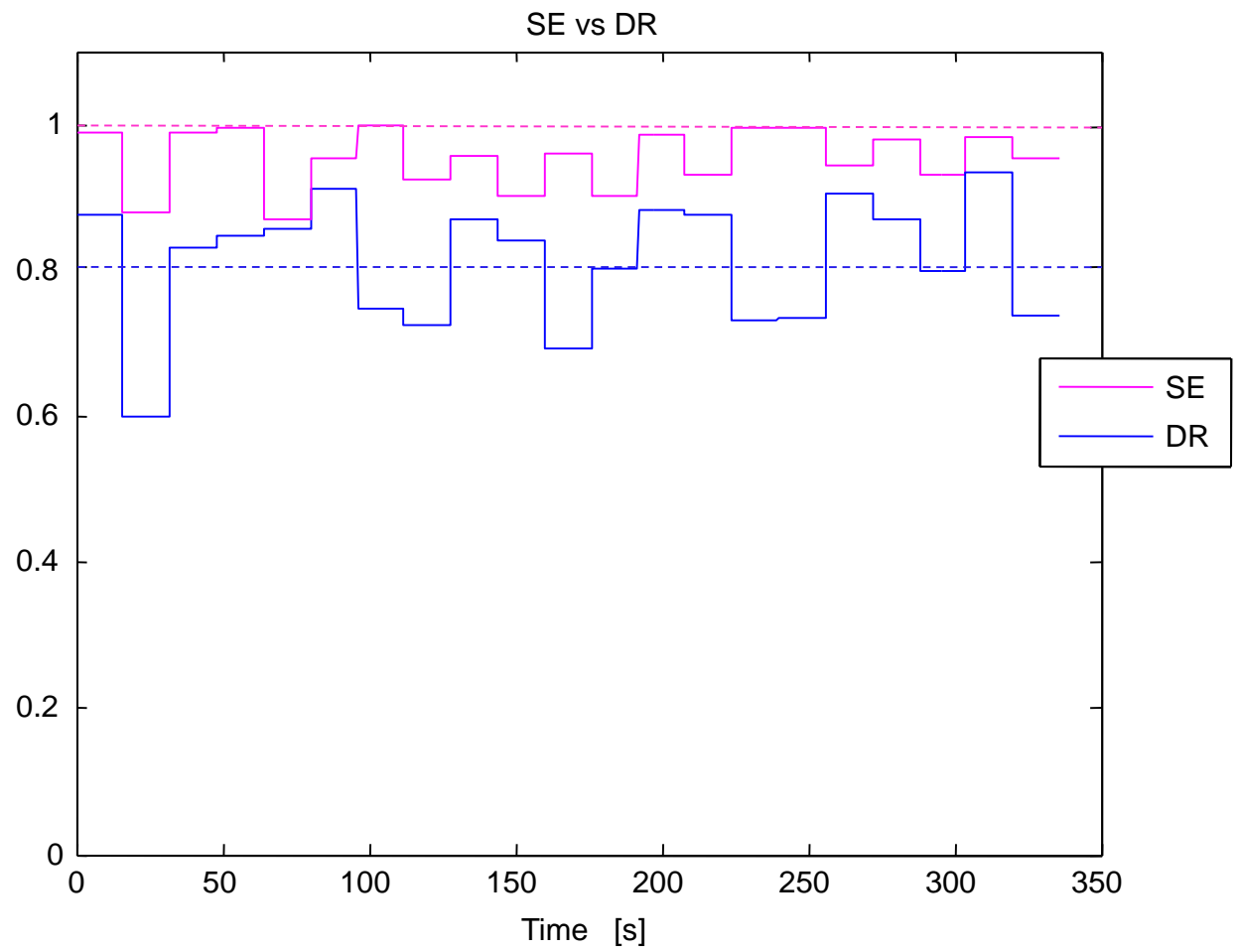

Figure 51. Estimated SE and DR along time for the APRM 1 signal. The purple dotted line located at 0.8 is the SE threshold (segments whose SE is above this line are unstable) whereas the blue dotted line at 1 is the DR threshold (segments whose DR is above this line are unstable).

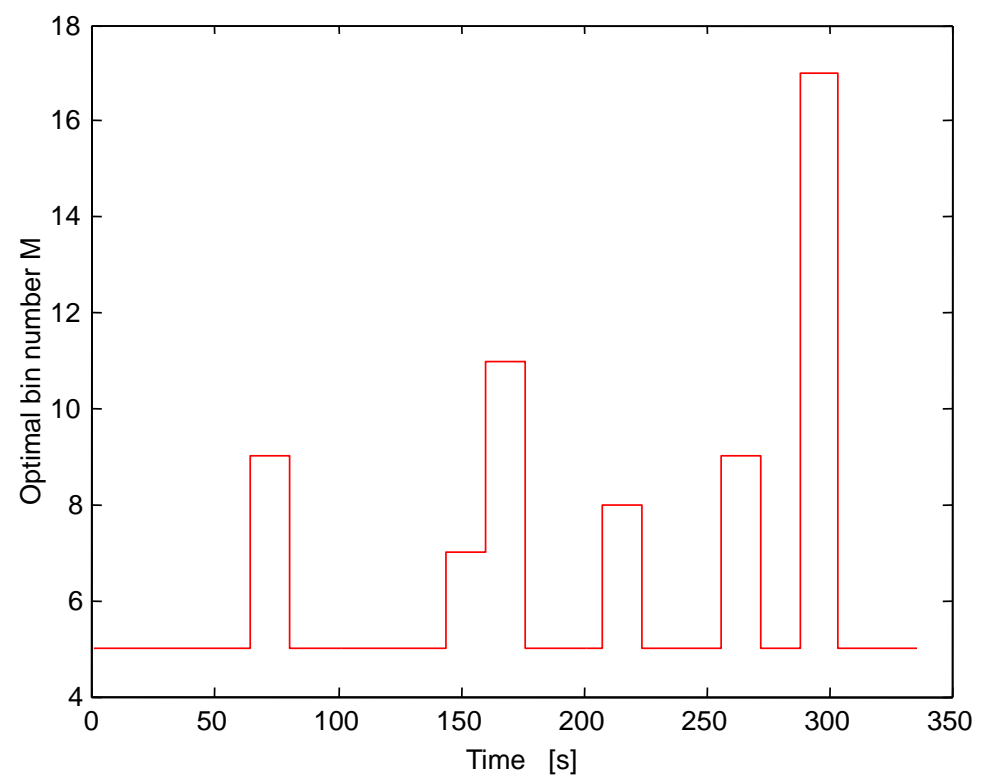

Figure 52. Estimated optimal number of bins $(5 \leq M \leq 20)$. 
Ultimately, the estimated SE, DR and oscillation frequency for the rest of the LPRMs of the studied Case 4 are shown in Table 6 (only average (Mean) and their standard deviations (std) values along all the studied segments are shown in Table 6). The estimated averaged values for the DR are in perfect agreement with those estimated by the different methodologies presented in the benchmark. The DR estimates point to the beginning of an incipient instability event whereas the SE estimates indicate a fully developed instability event in the BWR. Thus, it is naive to assume that we can infer the dynamics of a complex system such a BWR through an estimate of a linear parameter such as the DR alone. In spite of the contradictions of what these two parameters (SE and DR) are indicating, they nevertheless pinpoint to an instability event in the BWR core. Although the SE does this form the very beginning of the stability analysis.

Table 6. Average and standard deviations values for the SE, the DR and the oscillation frequency $\left(f_{0}\right)$ linked to instability of the Forsmark stability benchmark, Case 4, studied through Methodology 1 based on the iCEEMDAN.

\begin{tabular}{cllllll}
\hline Detectors & Mean SE & Std SE & Mean DR & Std DR & Mean fo & Std f \\
\hline APRM & 0.9553 & 0.0377 & 0.8136 & 0.0842 & 0.5279 & 0.0299 \\
LPRM 1 & 0.9527 & 0.0236 & 0.801 & 0.0765 & 0.519 & 0.0282 \\
LPRM 2 & 0.9564 & 0.0344 & 0.8007 & 0.1048 & 0.5101 & 0.03 \\
LPMR 3 & 0.9607 & 0.0222 & 0.8211 & 0.0778 & 0.5036 & 0.0202 \\
LPMR 4 & 0.9515 & 0.0268 & 0.7649 & 0.123 & 0.5116 & 0.0345 \\
LPRM 5 & 0.9323 & 0.0493 & 0.771 & 0.1269 & 0.5424 & 0.0317 \\
LPRM 6 & 0.9422 & 0.0304 & 0.765 & 0.1376 & 0.5444 & 0.0265 \\
LPRM 7 & 0.9409 & 0.0313 & 0.7623 & 0.0843 & 0.5513 & 0.0346 \\
LPMR 8 & 0.921 & 0.0411 & 0.6991 & 0.0873 & 0.5683 & 0.0509 \\
LPRM 9 & 0.9331 & 0.049 & 0.752 & 0.0966 & 0.5461 & 0.0384 \\
LPRM 10 & 0.9272 & 0.0429 & 0.7043 & 0.1315 & 0.574 & 0.0373 \\
LPRM 11 & 0.9224 & 0.0586 & 0.7527 & 0.0885 & 0.5513 & 0.0425 \\
LPRM 12 & 0.9074 & 0.0521 & 0.545 & 0.1649 & 0.5796 & 0.078 \\
LPRM 13 & 0.9436 & 0.0356 & 0.7753 & 0.1208 & 0.5462 & 0.0315 \\
LPRM 14 & 0.9334 & 0.0396 & 0.7783 & 0.0907 & 0.5386 & 0.0397 \\
LPRM 15 & 0.9428 & 0.0356 & 0.7569 & 0.1241 & 0.537 & 0.0408 \\
LPMR 16 & 0.9477 & 0.0331 & 0.7831 & 0.092 & 0.5362 & 0.0341 \\
LPMR 17 & 0.9449 & 0.0375 & 0.7683 & 0.089 & 0.5302 & 0.0486 \\
LPRM 18 & 0.9489 & 0.0375 & 0.7487 & 0.1392 & 0.5253 & 0.0362 \\
LPRM 19 & 0.915 & 0.0575 & 0.6295 & 0.1206 & 0.5111 & 0.0703 \\
LPRM 20 & 0.9152 & 0.0429 & 0.6834 & 0.1149 & 0.5631 & 0.0487 \\
LPMR 21 & 0.9227 & 0.0368 & 0.6841 & 0.1882 & 0.5777 & 0.0566 \\
LPRM 22 & 0.9026 & 0.0408 & 0.518 & 0.1275 & 0.5606 & 0.1011 \\
\hline
\end{tabular}




\section{LPRM signal from the Ringhals benchmark}

Now, the studied signal stems from the Ringhals stability benchmark case 9 cycle 14 . The studied signal is shown in Figure 53. The Methodology 1, based on the improved CEEMDAN and the SE is applied to this signal. This stability methodology splits the signal of interest in short segments of $15 \mathrm{~s}$, later the studied segment is decomposed through improved CEEMDAN into IMFs (or modes), the Hilbert-Huang Transform is calculated to obtain the instantaneous frequencies of the extracted IMFs. The IF of interest linked to instability (the energy of this IF of interest oscillates around $0.5 \mathrm{~Hz}$ ) is tracked. Later, the IMF associated to this IF is selected for SE calculation. Figure 54 shows the analysis of one studied segment that was decomposed through improved CEEMDAN into $\mathrm{n}$ IMFs and the IMF 3 was selected for further processing (because the IF 3, of this IMF (IMF 3) is linked to BWR instability, this key IF is shown in Figure 55).

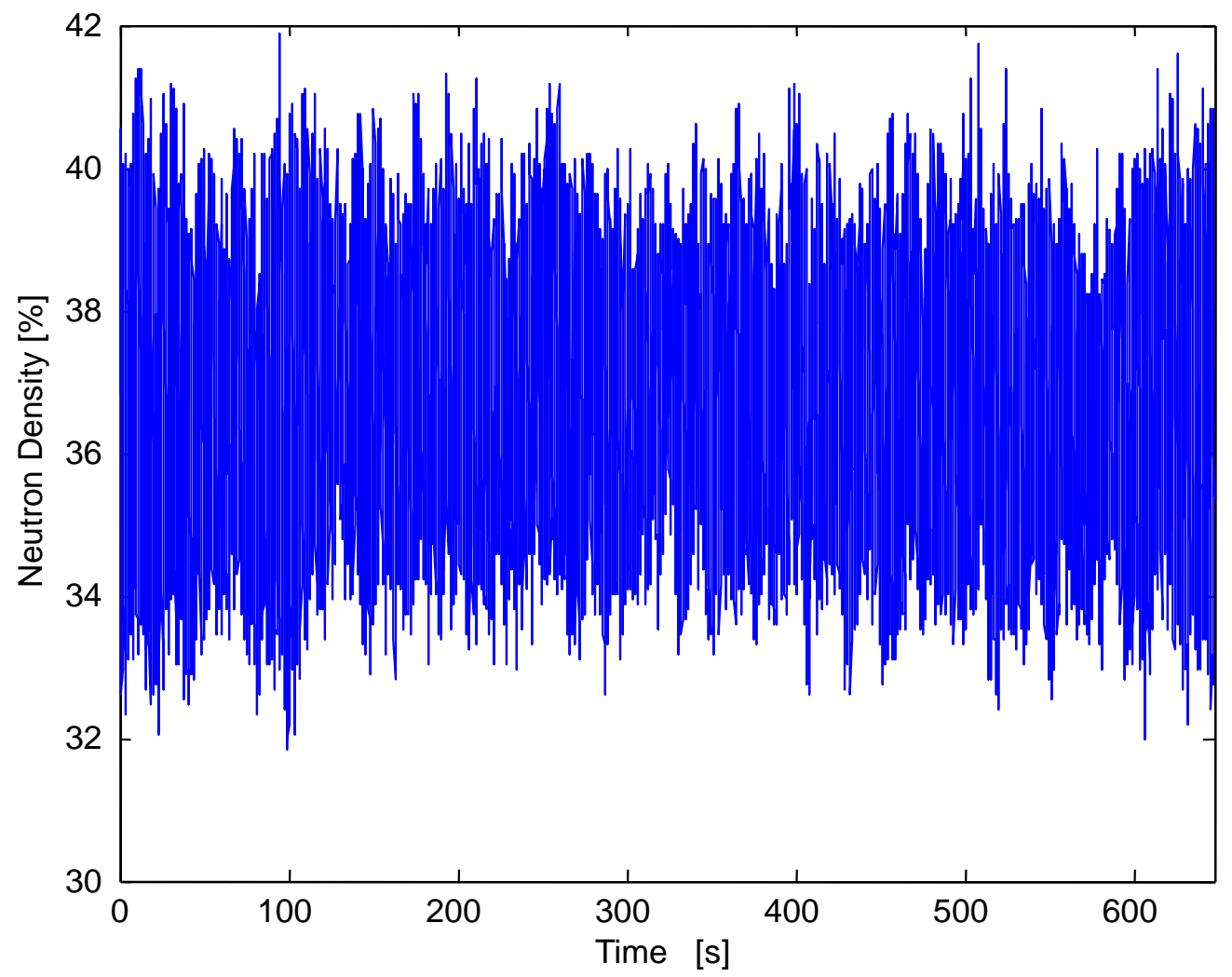

Figure 53. LPRM 1 from the Ringhals stability benchmark, case 9 cycle 14. 
Ringhals case 9 cycle 14 LPRM 1

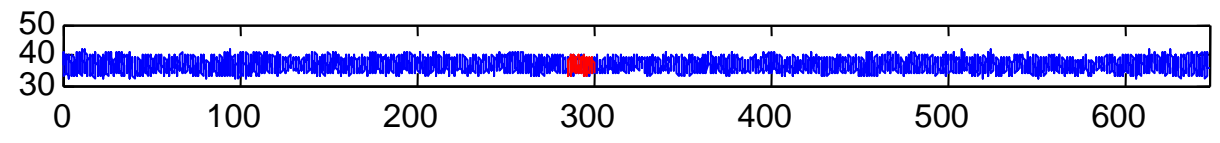

Segment of LPRM 1

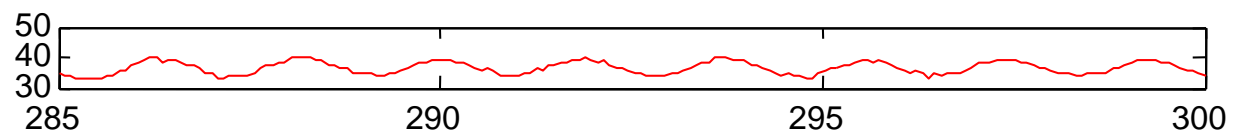

IMF $1+$ IMF 2

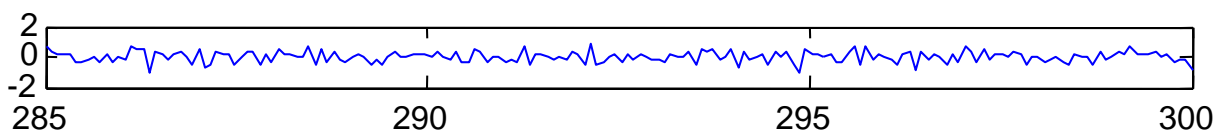

IMF 3

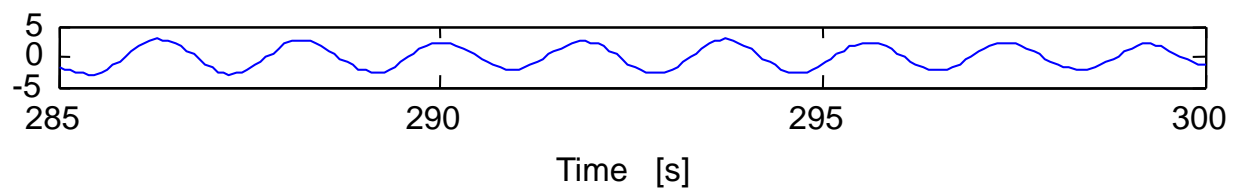

Figure 54. Improved CEEMDAN decomposition of one of the segments of the studied signal.

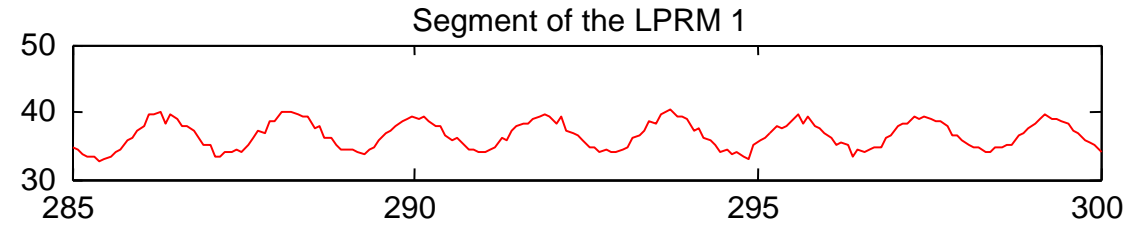

IMF 3 (linked to BWR instability)

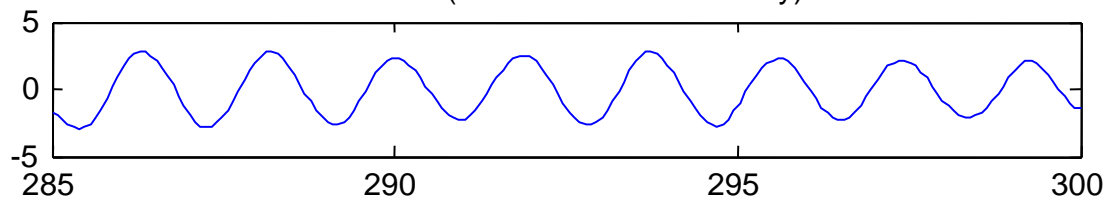

IF- IMF 3

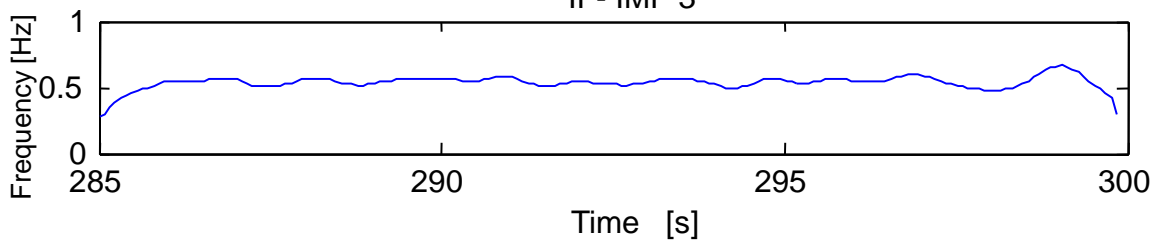

Figure 55. IF 3 linked to density wave instability. 
Figure 56 shows a power spectral density estimate of the extracted IMFs of the studied segment, to visualize the spectrum of the IMF 3 linked to instability and to observe again the improved CEEMDAN capabilities to compensate for mode mixing, which translates into less overlap of contiguous IMF spectra.

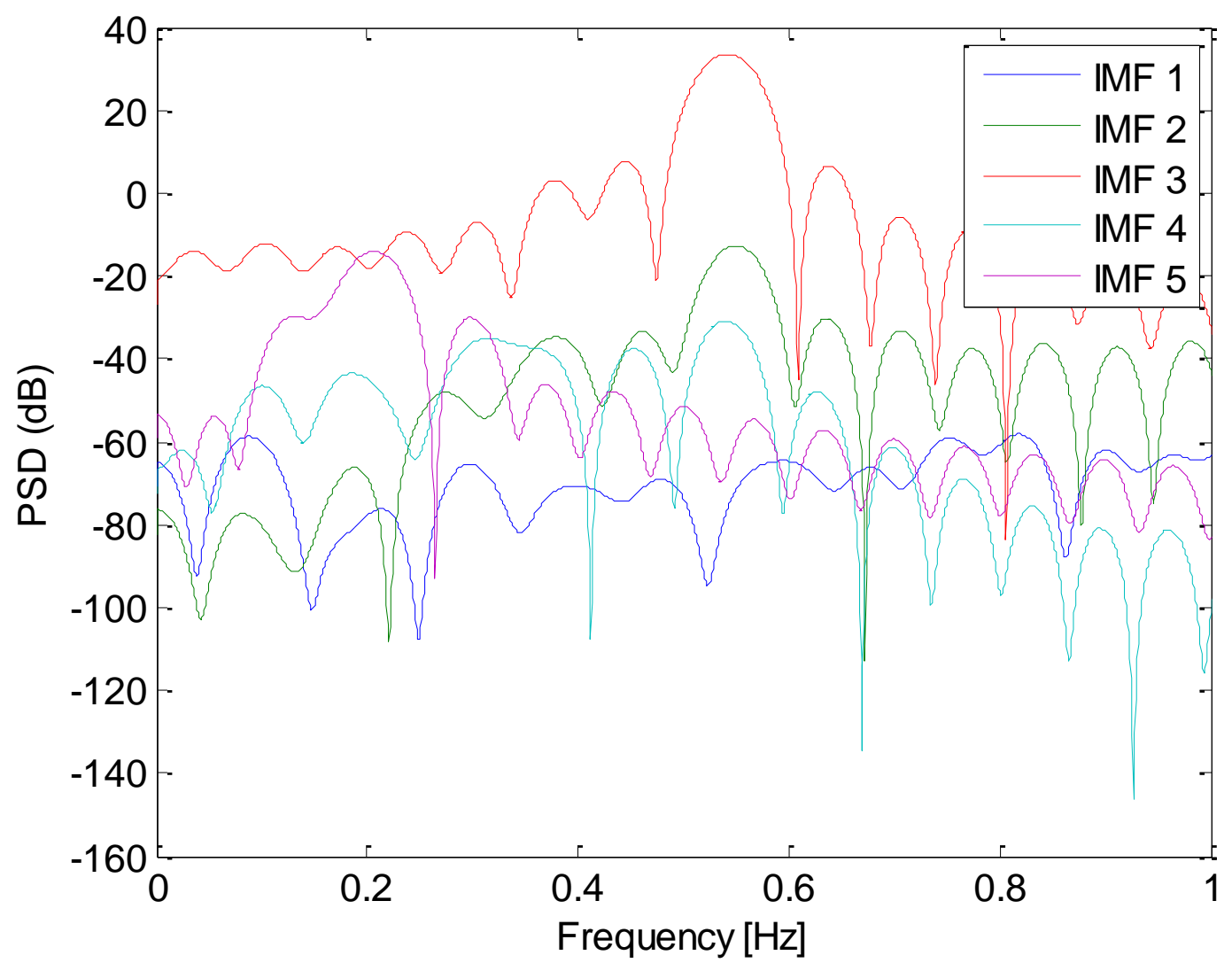

Figure 56. PSD estimate of the extracted IMFs of the studied segments.

Figure 57 shows the plot shows the plot of the estimated SE of all of the studied segments of the signal of interest. As before, in this figure, a DR estimate of the segments is also shown to illustrate the performance of the SE over the DR to analyze the stability of the studied signal. The instability thresholds for the DR and for the SE are the same as before. For this signal, again the SE indicates a fully developed unstable BWR behavior whereas the DR is pointing to an early development of an instability event (a quasi-instable event), because the average DR is high (not exactly one, but approaching it). Again, the high SE estimates of the studied segments of this LRPM 1 signal are clearly indicating an out of the ordinary BWR behavior. The estimated number of bins $\mathrm{M}$ remained throughout most the simulation constant at 7 bins. The proposed stability monitor, given in Methodology 1, proves again to be suitable to detect unstable or not ordinary BWR behavior prior further growth of such unforeseen unstable events, that may in the worst case scenarios, trigger increasing power oscillations beyond the nominal BWR constraints. Thus, it is necessary to be able to detect any incipient unstable events as fast as possible. 


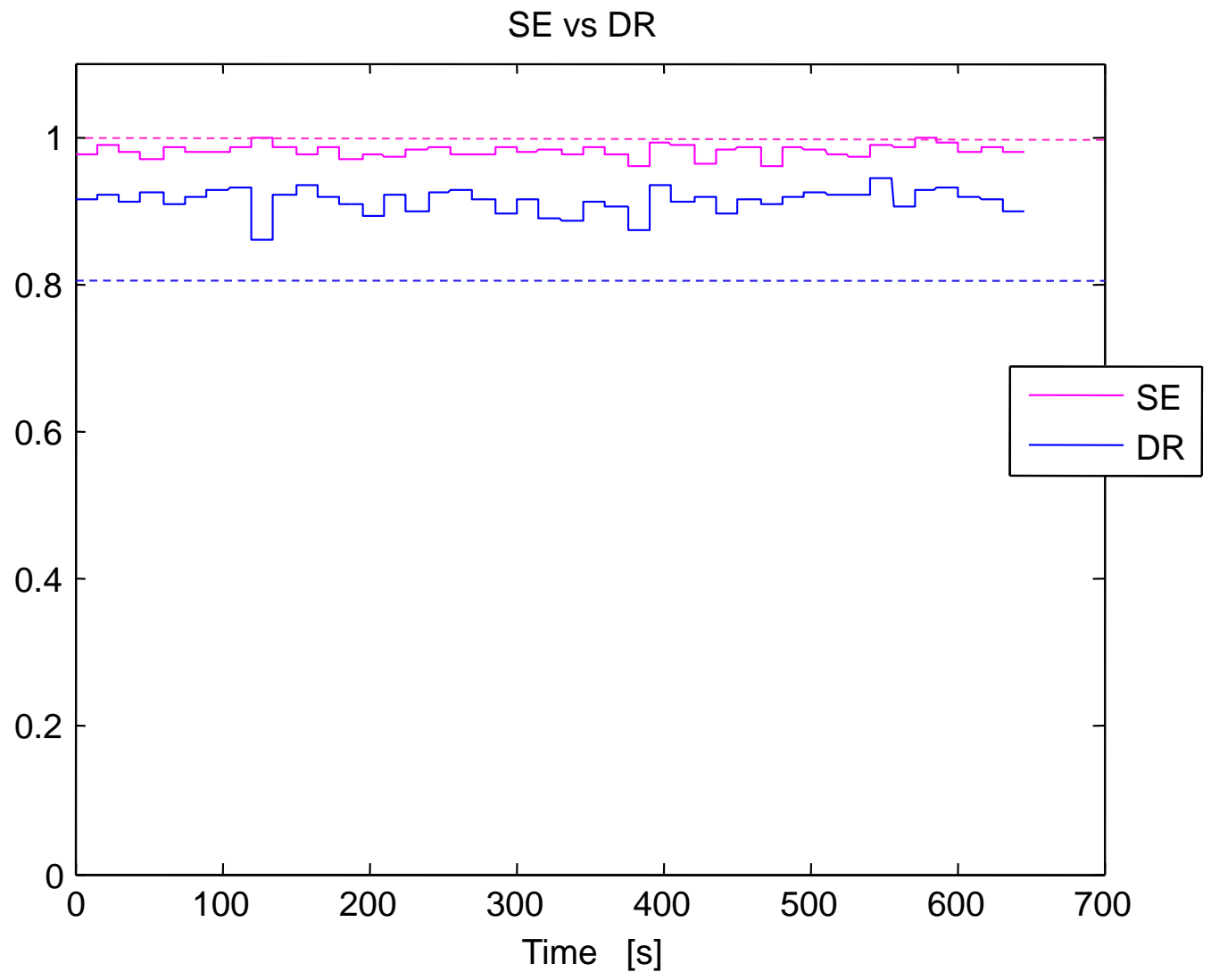

Figure 57. Estimated Shannon Entropy (SE) and Decay Ratio (DR) estimate along time for the LRPM 1 signal, Case 9 from Ringhals stability benchmark. The purple dotted line located at 0.8 is the SE threshold (segments whose SE is above this line are unstable) whereas the blue dotted line at 1 is the DR threshold (segments whose DR is above this line are unstable).

Finally, the estimated SE, DR and oscillation frequency for the rest of the LPRMs of the studied Cycle 14 Case 9 are shown in Table 7 (only average values and standard deviations along all the studied segments are shown in Table 7). The entire case consists of a total of 72 LPRMs distributed on two different floors or levels (2 and 4) within the BWR core. In Table 7, only the analysis of the floor number 2 is studied. This floor consists of 36 LPRM detectors marked by odd numbers.

The estimated DR results of this studied case and shown in Table 7, where in most LPRMs high and apparently this case exhibits and out-of-phase oscillation, which will be scoped in detail once Methodology 2 based on the NA-MEMD is used to perform a multivariate analysis of this particular case. Overall, The high SE estimated values, clearly indicate a fully developed unstable behavior of this case. Thus, the studied BWR floor 2 is unstable. The high DR estimates ( but still not above the 1, which is the stability threshold that must be exceeded by the DR to trigger BWR peril alarms) although high and depicting that there is something unusual going on in the BWR core. But, the estimates are not high enough to 
trigger BWR protection alarms to warn the operators whereas the SE estimates would have trigger such BWR protection circuits.

Table 7. Average and standard deviations values for the SE, the DR and the oscillation frequency $\left(\mathrm{f}_{0}\right)$ linked to instability of the Ringhals stability benchmark Case 9 Cycle 14 studied through the Methodology 1 based on the improved CEEMDAN.

\begin{tabular}{|c|c|c|c|c|c|c|}
\hline Detectors & Mean SE & Std SE & Mean DR & Std DR & Mean fo & Std f $\mathbf{f}_{0}$ \\
\hline LPRM 1 & 0.9809 & 0.0084 & 0.9132 & 0.0161 & 0.5164 & 0.0248 \\
\hline LPRM 3 & 0.9826 & 0.0069 & 0.9122 & 0.0171 & 0.5153 & 0.0226 \\
\hline LPRM 5 & 0.9834 & 0.0094 & 0.9102 & 0.0162 & 0.5157 & 0.0246 \\
\hline LPRM 7 & 0.9877 & 0.0153 & 0.8897 & 0.0367 & 0.5149 & 0.0243 \\
\hline LPRM 9 & 0.9854 & 0.0082 & 0.9135 & 0.0184 & 0.5139 & 0.0266 \\
\hline LPRM 11 & 0.9823 & 0.0078 & 0.9134 & 0.0172 & 0.5175 & 0.0248 \\
\hline LPRM 13 & 0.9820 & 0.0106 & 0.9108 & 0.0214 & 0.5169 & 0.0219 \\
\hline LPRM 15 & 0.9856 & 0.0088 & 0.9091 & 0.0179 & 0.5106 & 0.0270 \\
\hline LPRM 17 & 0.9883 & 0.0080 & 0.9006 & 0.0221 & 0.5188 & 0.0241 \\
\hline LPRM 19 & 0.9621 & 0.0436 & 0.8332 & 0.0778 & 0.5180 & 0.0274 \\
\hline LPRM 21 & 0.9814 & 0.0270 & 0.8693 & 0.0506 & 0.5218 & 0.0267 \\
\hline LPRM 23 & 0.9862 & 0.0149 & 0.8909 & 0.0301 & 0.5174 & 0.0248 \\
\hline LPRM 25 & 0.9841 & 0.0122 & 0.8997 & 0.0273 & 0.5125 & 0.0267 \\
\hline LPRM 27 & 0.9869 & 0.0142 & 0.8951 & 0.0352 & 0.5136 & 0.0281 \\
\hline LPRM 29 & 0.9653 & 0.0509 & 0.8309 & 0.0762 & 0.5186 & 0.0364 \\
\hline LPRM 31 & 0.9500 & 0.0441 & 0.8106 & 0.0820 & 0.5049 & 0.0348 \\
\hline LPRM 33 & 0.9429 & 0.0409 & 0.6562 & 0.1321 & 0.4868 & 0.0286 \\
\hline LPRM 35 & 0.9630 & 0.0352 & 0.7145 & 0.2115 & 0.5020 & 0.0365 \\
\hline LPRM 37 & 0.9771 & 0.0203 & 0.8538 & 0.0490 & 0.5124 & 0.0272 \\
\hline LPRM 39 & 0.9598 & 0.0335 & 0.7558 & 0.0766 & 0.5062 & 0.0338 \\
\hline LPRM 41 & 0.9141 & 0.0637 & 0.5868 & 0.2425 & 0.4987 & 0.0423 \\
\hline LPRM 43 & 0.8814 & 0.0672 & 0.4893 & 0.2241 & 0.4922 & 0.0386 \\
\hline LPRM 45 & 0.9858 & 0.0124 & 0.8496 & 0.0415 & 0.5126 & 0.0265 \\
\hline LPRM 47 & 0.9854 & 0.0094 & 0.8816 & 0.0284 & 0.5071 & 0.0242 \\
\hline LPRM 49 & 0.9807 & 0.0091 & 0.9110 & 0.0173 & 0.5102 & 0.0279 \\
\hline LPRM 51 & 0.9771 & 0.0086 & 0.9120 & 0.0133 & 0.5121 & 0.0218 \\
\hline LPRM 53 & 0.9823 & 0.0077 & 0.9096 & 0.0184 & 0.5154 & 0.0262 \\
\hline LPRM 55 & 0.9868 & 0.0091 & 0.8974 & 0.0250 & 0.5204 & 0.0218 \\
\hline LPRM 57 & 0.9804 & 0.0076 & 0.9061 & 0.0143 & 0.5195 & 0.0188 \\
\hline LPRM 59 & 0.9771 & 0.0087 & 0.9084 & 0.0149 & 0.5126 & 0.0223 \\
\hline LPRM 61 & 0.9765 & 0.0101 & 0.9126 & 0.0149 & 0.5140 & 0.0229 \\
\hline LPRM 63 & 0.9764 & 0.0089 & 0.9123 & 0.0137 & 0.5112 & 0.0245 \\
\hline LPRM 65 & 0.9805 & 0.0085 & 0.9059 & 0.0185 & 0.5117 & 0.0268 \\
\hline LPRM 67 & 0.9832 & 0.0113 & 0.9054 & 0.0202 & 0.5149 & 0.0223 \\
\hline LPRM 69 & 0.9817 & 0.0093 & 0.9023 & 0.0184 & 0.5155 & 0.0197 \\
\hline LPRM 71 & 0.9831 & 0.0073 & 0.9029 & 0.0181 & 0.5156 & 0.0253 \\
\hline
\end{tabular}




\section{APRM laguna verde}

The studied signal that is now studied stems from an instability event that happened in Laguna Verde, in the year 1995. This signal is shown in Figure 58 and was obtained via the integral Information Process System (IIPS). The channel A of the APRM trace shows no unstable behavior at 3:28:00 h. The value closure was initiated at 03:28:20 h. A small core flow reduction was noticeable $40 \mathrm{~s}$ later, and the APRM-A trace depicts signs of instability although the variations in the magnitude of the signal remained within the noise level. As the valve continued to close, the APRM-A trace shows clear unstable behavior starting at 03:30:30 $\mathrm{h}$. The valve reached the minimum position at 03:31:30 $\mathrm{h}$. The valve reached the minimum position at 03:31:30 $\mathrm{h}$, and the oscillations continued without any significant increase in their growth rate. The operator attempted to stabilize the power level by increasing the core flow opening the vales at 03:33:20 h. As a result of increasing the core flow, the oscillation started to decay at 03:34:40 h. At 03:35:20 h the oscillation reached $3 \%$ of amplitude, when the reactor was manually scrammed (see the red boxes in Figure $58)$.

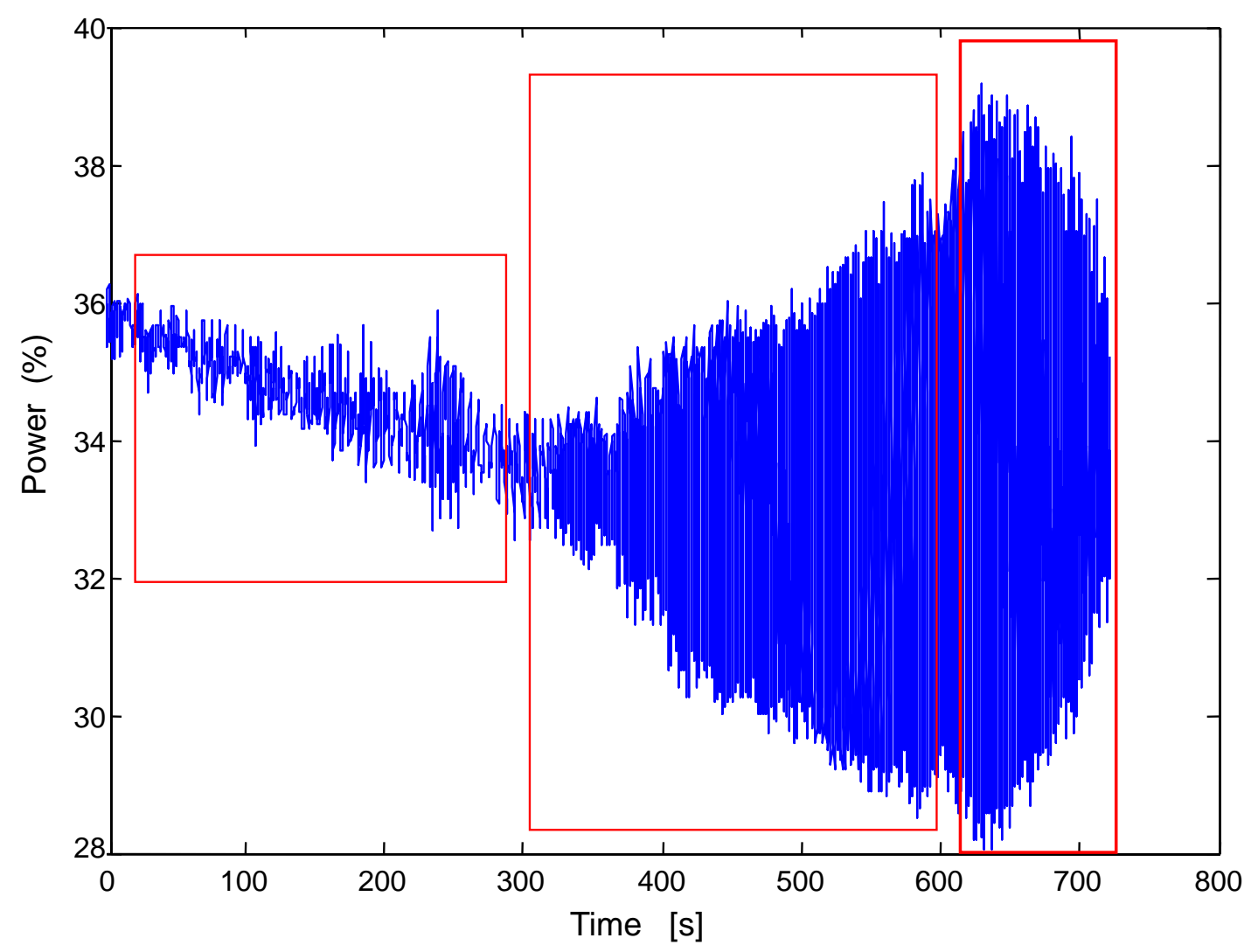

Figure 58 . Laguna Verde (LV) APRM signal of an unstable event that occurred in 1995. 
As before, Figure 59 shows the decomposition of one of the segments of the studied Laguna Verde APRM, decomposed according to Methodology 1 based on the improved CEEMDAN. The IMF linked to BWR instability in this case is the IMF 1 , see its instantaneous frequency (IF 1) oscillating around $0.5 \mathrm{~Hz}$. This IF 1 is shown in Figure 60 and also the power spectral density estimates of all the extracted IMFs are shown in Figure 61. Observe that the PSD of IMF 2 is slightly mixed with the PSD estimate of IMF 1, but the spectral energetic content of IMF 2 is negligible in comparison with that of IMF 1.
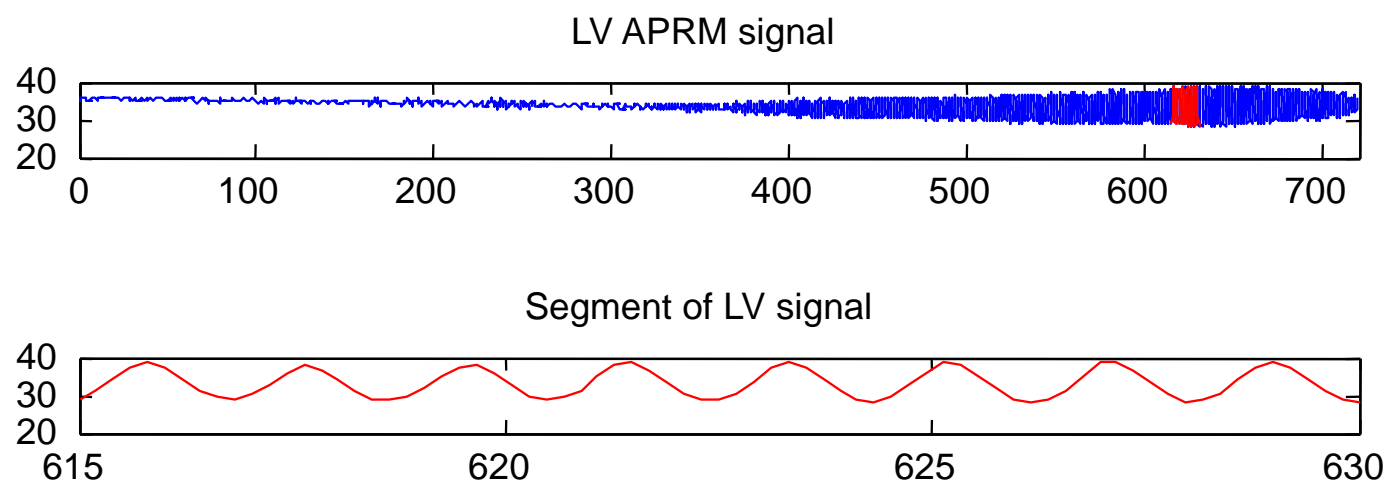

IMF 1

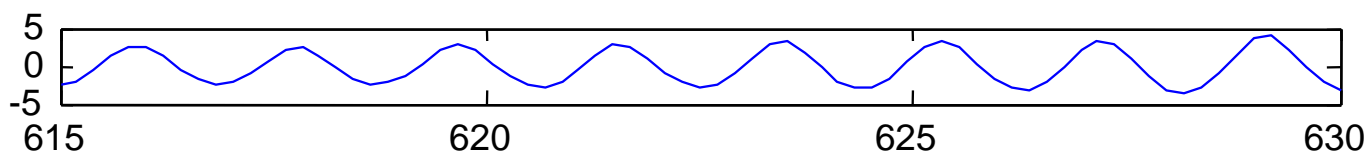

IMF 2

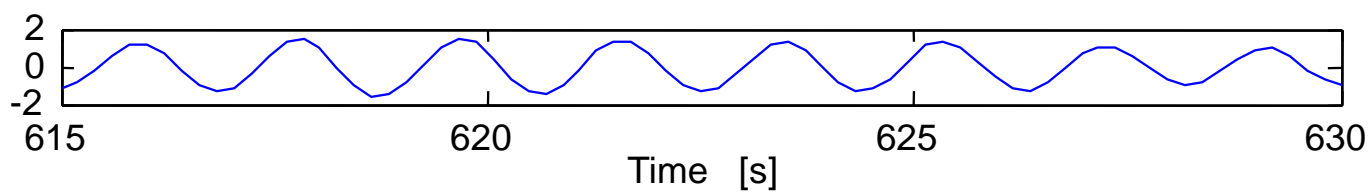

Figure 59. Improved CEEMDAN of one of the segments of the APRM signal of a Laguna Verde density wave instability. Only the first 2 IMFs are shown in this plot. 

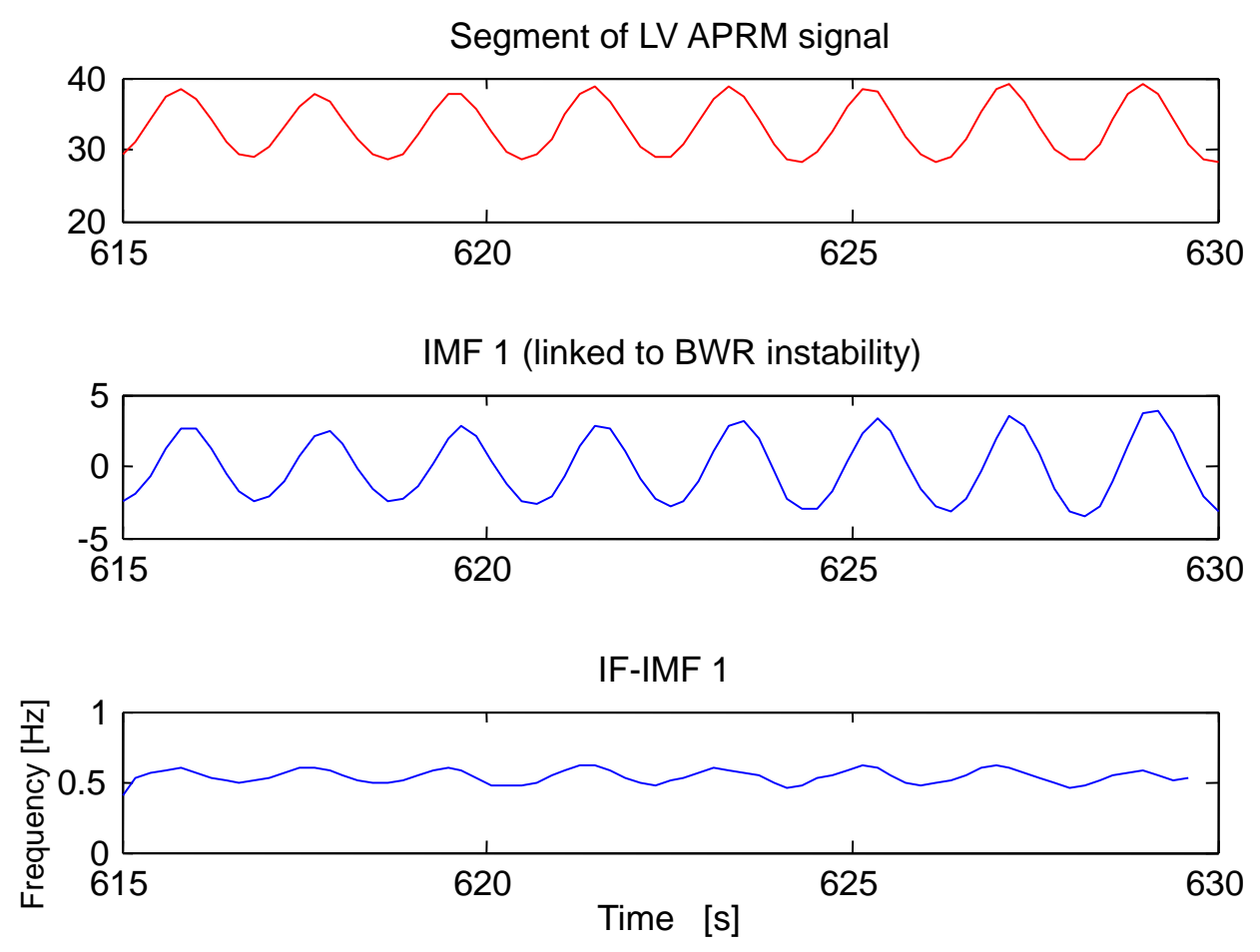

Figure 60. IF 1 linked to BWR instability. The time series of IF 1 oscillates in a quasisinusoidal manner around $0.5 \mathrm{~Hz}$ (the region of interest for BWR instability events).

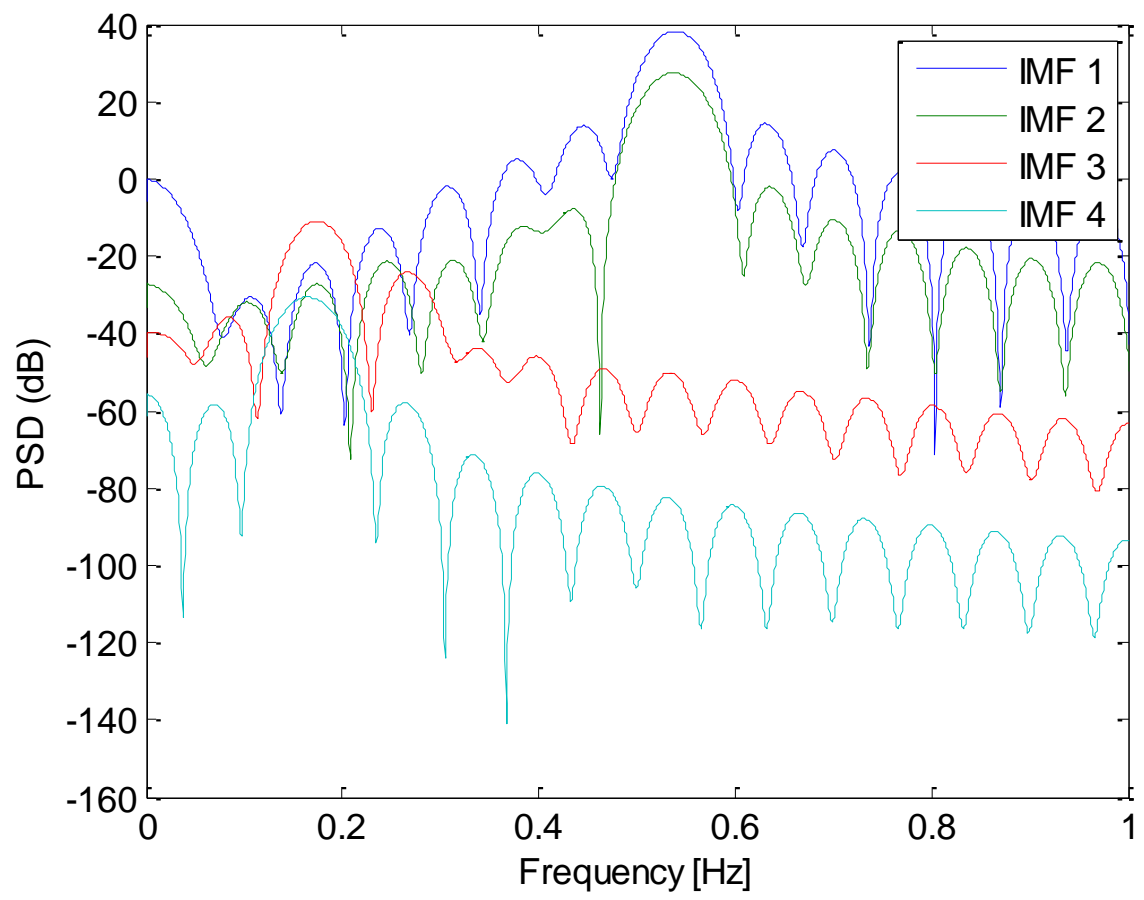

Figure 61. PSD estimate of the extracted IMFs of the studied segment through the improved CEEMDAN method. 
Figure 62 shows the SE and DR estimates along all the studied segments of the APRM signal of interest. Prior the first $300 \mathrm{~s}$ of the signal, the DR oscillates between stability and instability. But, it is cumbersome to infer the dominant DR value due to its strong discontinuous jumps between stability and instability. However, after the $300 \mathrm{~s}$ mark, the $\mathrm{DR}$ is high and greater that its threshold value $(\mathrm{DR}=1)$ and remains as such (and oscillating around 1.1) throughout the rest of the simulation. Thus, the DR indicates unstable BWR behavior but only after the 300 s mark.

The SE estimate is highly more consistent than the DR prior the $300 \mathrm{~s}$ mark, because the SE clearly indicates unstable behavior (whereas the DR is unable to differentiate between the two) and after the $300 \mathrm{~s}$ mark, the SE slightly oscillates around 1 (and not in a dramatic way as the DR does). Nevertheless, the SE always indicates unstable BWR behavior, long before the DR is able to detect it. Thus, the SE is capable of indicating unstable behavior prior any further growth in power of the unstable oscillation within the core whereas the DR is only able to detect instability (without bias) once the unstable oscillation is fully sustained and powerful enough to damage the core. The optimal number of bins for this case remained most of the simulation constant at 10 .

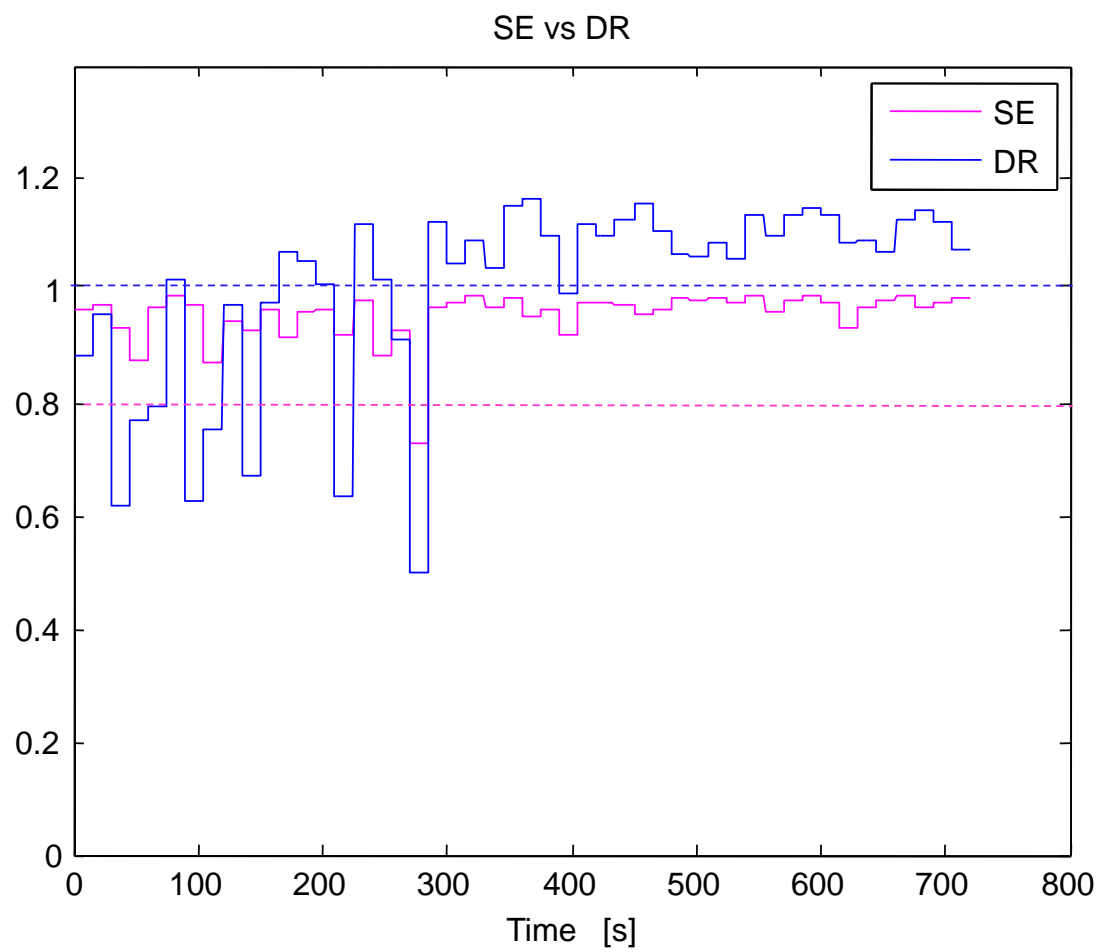

Figure 62. Estimated Shannon Entropy (SE) and Decay Ratio (DR) estimate along time for the APRM signal. The purple dotted line located at 0.8 is the SE threshold (segments whose SE is above this line are unstable) whereas the blue dotted line at 1 is the DR threshold (segments whose DR is above this line are unstable). 
Finally, Table 8 shows the mean SE, DR and instantaneous frequency averaged along all the segments of the signal of interest.

Table 8. Average and standard deviations values for the SE, the DR and the oscillation frequency $\left(\mathrm{f}_{0}\right)$ linked to instability of the Laguna Verde APRM signal studied through the Methodology 1 based on the iCEEMDAN.

\begin{tabular}{ccccccc}
\hline Detector & Mean SE & Std SE & Mean DR & Std DR & Mean fo & Std fo \\
\hline APRM & 0.9592 & 0.0444 & 1.0079 & 0.1655 & 0.5385 & 0.0158 \\
\hline
\end{tabular}

\section{Stability analysis of the chosen real cases through Methodology 2}

The stability methodology 2 is applied with the next following cases of nuclear power plants (NPP):

I. Multidimensional analysis of the already mentioned Case 4 of the Forsmark stability benchmark.

II. Multidimensional analysis of the also mentioned Case 9 Cycle 14 of the Ringhals stability benchmark.

Regarding Laguna Verde instability event, the methodology 2 can also be applied. However, the signals from 96 LPRMs monitoring the core are not available for this specific instability phenomenon.

\section{LPRMs signals form Forsmark benchmark}

Now, the case 4 of the Forsmark stability benchmark is going to be studied with the stability Methodology 2 based on the NA-MEMD in a multivariate way $\mathrm{m}=3$ independent channels of noise to mitigate mode mixing. In here, the ensemble of LPRM signals is considered in the NA-MEMD and a local estimation of SE and of the DR (calculated according to Olvera-Guerrero et al., 2017) are computed based on the IMFs associated to the instability event (the oscillatory IMF around $0.5 \mathrm{~Hz}$ ). Figure 63 shows the IMFs linked to BWR instability.

Exploiting the time alignment property of the NA-MEMD, these IMFs of interest are located at the same level of decompositions, in this case the IMFs of interest are located at the fourth level of the NA-MEMD decomposition (IMFs number 4). We highlight that in Figure 63. the IMFs of interest linked to instability are in-phase among them. The instantaneous frequencies (IFs number 4$)$ around the region of interest $(0.5 \mathrm{~Hz})$ of these IMFs of interest are shown in Figure 64. Later, Figure 65 shows the estimated SE locally for each IMF of interest (IMFs number 4). However, for simplicity, only a sample of 4 IMFs are shown in this plot, the selected IMFs are LPRM 1, LPRM 7, LPRM 11 and LPRM 21. Also, the DR (depicted in Figure 66 and estimated in the same way as before) is estimated locally for each IMF but again, only 4 IMFs (the aforementioned 4 LPRM signals) are shown in such figure. In the multivariate scenario, overall the BWR is unstable because of the high SE estimates along time, in spite of 4 segments that had an SE below the stability threshold ( $\mathrm{SE}<0.8$ ). Thus, again from the very beginning of the simulation, 
the SE is able to detect an unusual BWR unstable behavior. The DR in the multivariate case prior the $150 \mathrm{~s}$ mark is apparently stable and after this $150 \mathrm{~s}$ mark, it fluctuates around 0.75 , the DR estimate is high but not high enough to trigger the BWR warning mechanisms and thus the DR indicates quasi-instability.

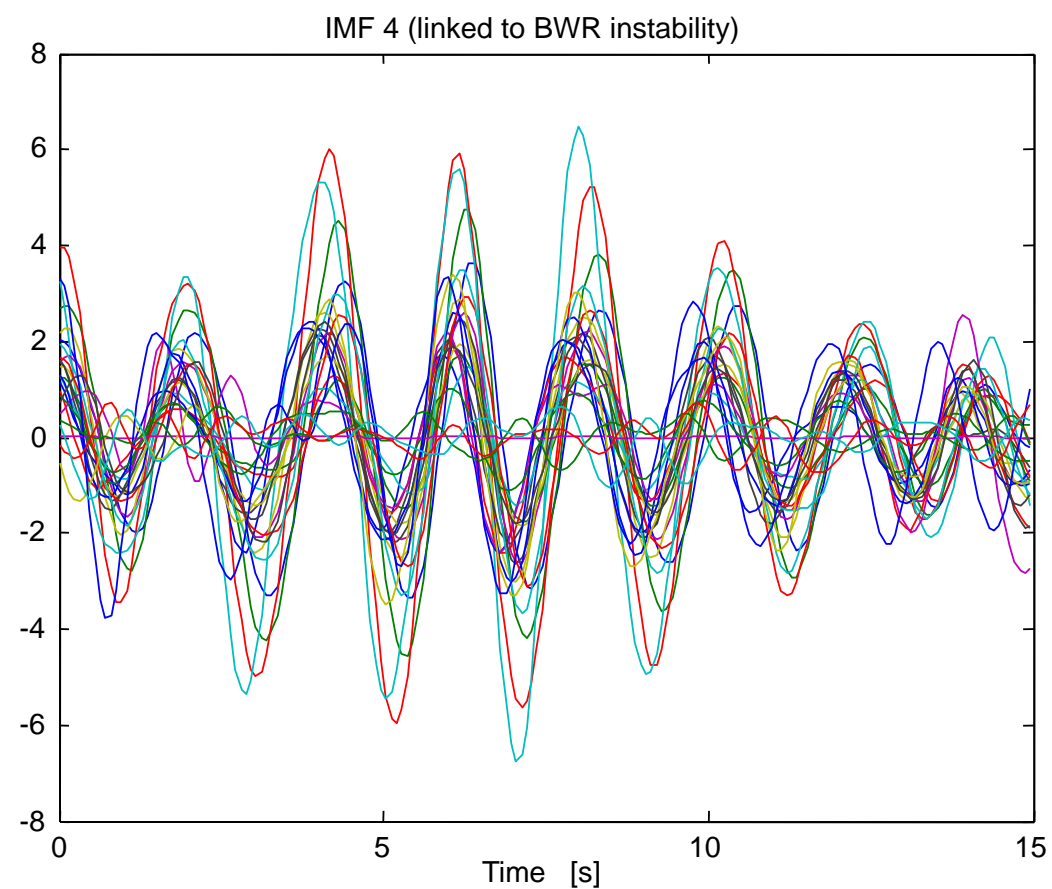

Figure 63. NA-MEMD applied to a short time segment of Case 4 of the Forsmark instability benchmark.

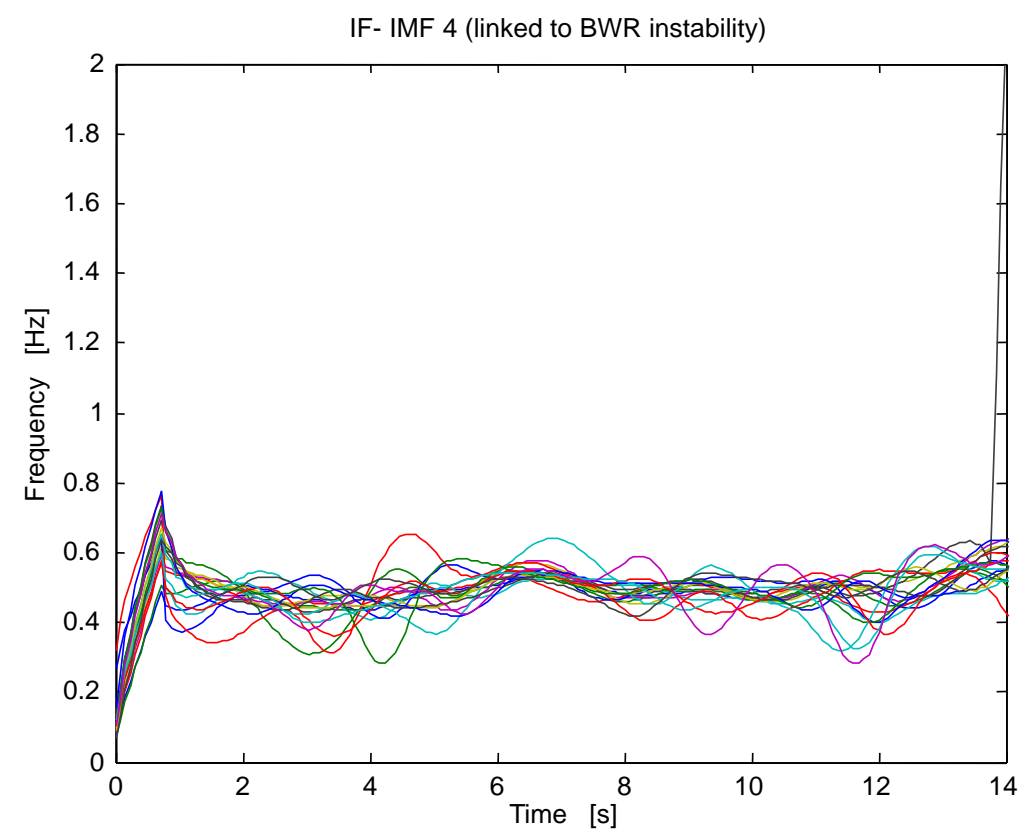

Figure 64. Frequencies IFs (IFs 4) linked to density wave instability. 


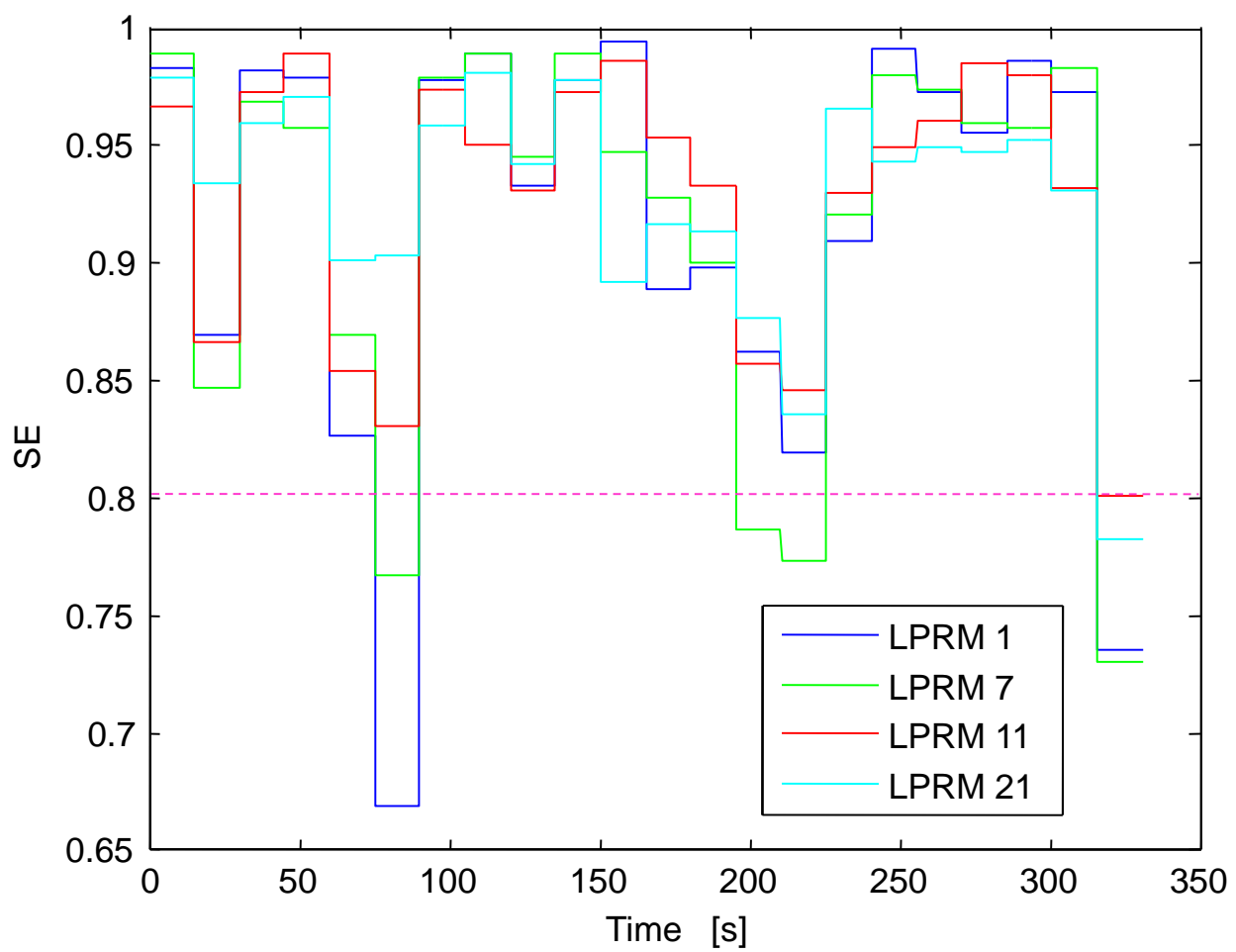

Figure 65. Local SE estimate along time for the selected 4 LPRM sample. The threshold SE bar is located at the same locus as before.

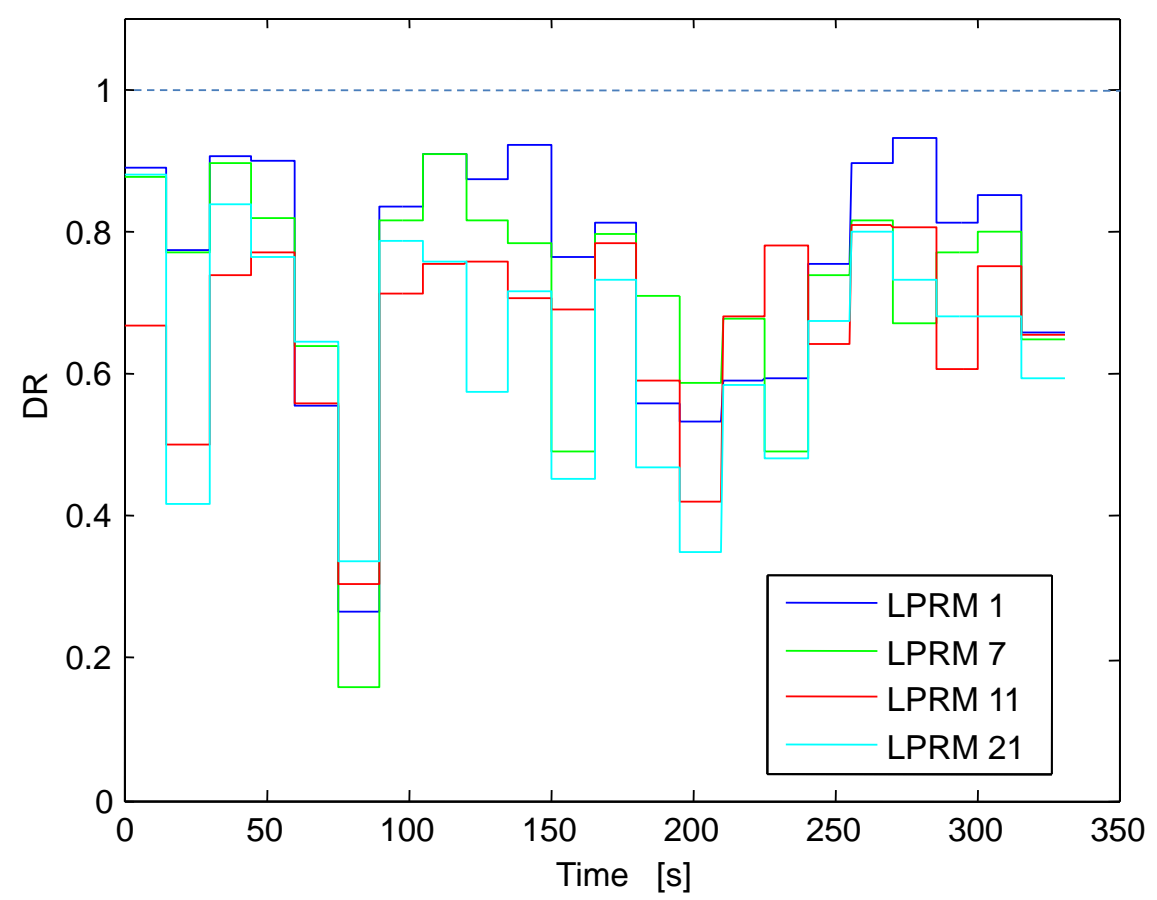

Figure 66. Local DR estimate along time for the selected 4 LPRM sample. 
Finally, Table 9 shows the SE, DR and fo (all of them calculated locally) of the entire studied Case 4 of the Forsmark stability benchmark, the APRM was ignored for this analysis. The estimated parameters are similar to those that stem from the univariate analysis performed through the Methodology 1 in Table 6 of this case (the estimates in Table 9 are similar to those depicted in Table 6 and within the 10\% difference).

Table 9. Average and standard deviation values for the SE, the DR and the oscillation frequency $\left(\mathrm{f}_{0}\right)$ linked to instability of the Forsmark benchmark stability Case 4 studied via stability methodology 2 based on NA-MEMD.

\begin{tabular}{ccccccc}
\hline Detectors & Mean SE & Std SE & Mean DR & Std DR & Mean f & Std f $_{\mathbf{0}}$ \\
\hline LPRM 1 & 0.9208 & 0.0816 & 0.7669 & 0.1417 & 0.4754 & 0.0283 \\
LPRM 2 & 0.9220 & 0.0842 & 0.7670 & 0.1526 & 0.4867 & 0.0250 \\
LPMR 3 & 0.9164 & 0.0924 & 0.7791 & 0.1457 & 0.4875 & 0.0260 \\
LPMR 4 & 0.9034 & 0.1001 & 0.7551 & 0.1476 & 0.4867 & 0.0214 \\
LPRM 5 & 0.9278 & 0.0762 & 0.7328 & 0.1585 & 0.5030 & 0.0373 \\
LPRM 6 & 0.9234 & 0.0783 & 0.7383 & 0.1338 & 0.5034 & 0.0357 \\
LPRM 7 & 0.9176 & 0.0789 & 0.7232 & 0.1232 & 0.5012 & 0.0368 \\
LPMR 8 & 0.9160 & 0.0761 & 0.6595 & 0.1511 & 0.5047 & 0.0447 \\
LPRM 9 & 0.9241 & 0.0767 & 0.6749 & 0.1703 & 0.5016 & 0.0355 \\
LPRM 10 & 0.9127 & 0.0700 & 0.6131 & 0.1748 & 0.5129 & 0.0425 \\
LPRM 11 & 0.9278 & 0.0618 & 0.6466 & 0.1482 & 0.4980 & 0.0395 \\
LPRM 12 & 0.9167 & 0.0450 & 0.5177 & 0.1378 & 0.5163 & 0.0636 \\
LPRM 13 & 0.9218 & 0.0721 & 0.7076 & 0.1327 & 0.5020 & 0.0292 \\
LPRM 14 & 0.9130 & 0.0756 & 0.6945 & 0.1537 & 0.5047 & 0.0300 \\
LPRM 15 & 0.9162 & 0.0785 & 0.7021 & 0.1281 & 0.5028 & 0.0385 \\
LPMR 16 & 0.9108 & 0.0889 & 0.7145 & 0.1088 & 0.5018 & 0.0299 \\
LPMR 17 & 0.9235 & 0.0814 & 0.7331 & 0.1506 & 0.4927 & 0.0242 \\
LPRM 18 & 0.9233 & 0.0851 & 0.7158 & 0.1693 & 0.4990 & 0.0282 \\
LPRM 19 & 0.9235 & 0.0670 & 0.6521 & 0.1686 & 0.4947 & 0.0477 \\
LPRM 20 & 0.9060 & 0.0884 & 0.6337 & 0.1861 & 0.5020 & 0.0428 \\
LPMR 21 & 0.9256 & 0.0668 & 0.6290 & 0.1512 & 0.5037 & 0.0413 \\
LPRM 22 & 0.8900 & 0.0593 & 0.4413 & 0.1466 & 0.5118 & 0.0840 \\
\hline
\end{tabular}

\section{LPRMs from Ringhals benchmark}

Now, the Case 9 cycle 14 of the Ringhals stability benchmark is studied through Methodology 2 based on the NA-MEMD. Figure 67 shows the NA-MEMD decomposition ( with 3 independent channels of noise to compensate for mode mixing) of one of the signal segments, the IMF (IMF 4) linked to density wave instability in shown in this figure and the type of observed oscillation is an out-of-phase one. These type of oscillations can only be observed locally at the LPRM level because at the APRM level (an APRM signal is an average of $n$ LPRMs) the averaging might cancel data, if the signals that participate in the average have ideal phase differences of 180 degrees among them. 
Figure 68 shows the instantaneous frequencies IFs (IF 4) of the IMFs (IMF at the 4 level of NA-MEMD decomposition) associated to BWR instability, all of the IFs oscillate around $0.5 \mathrm{~Hz}$ in a quasi sinusoid way. Figure 69 shows the SE estimates along time of a sample of 4 LPRMs that were selected at random, the selected LPRMs were: LPRM 1, LPRM 10, LPRM 20 and LPRM 29. The SE estimates along time were high (beyond the SE stability threshold located at $\mathrm{SE}=0.8$ ) throughout the time span of the simulation for the 4 chosen LPRMs, thus the BWR is clearly unstable.

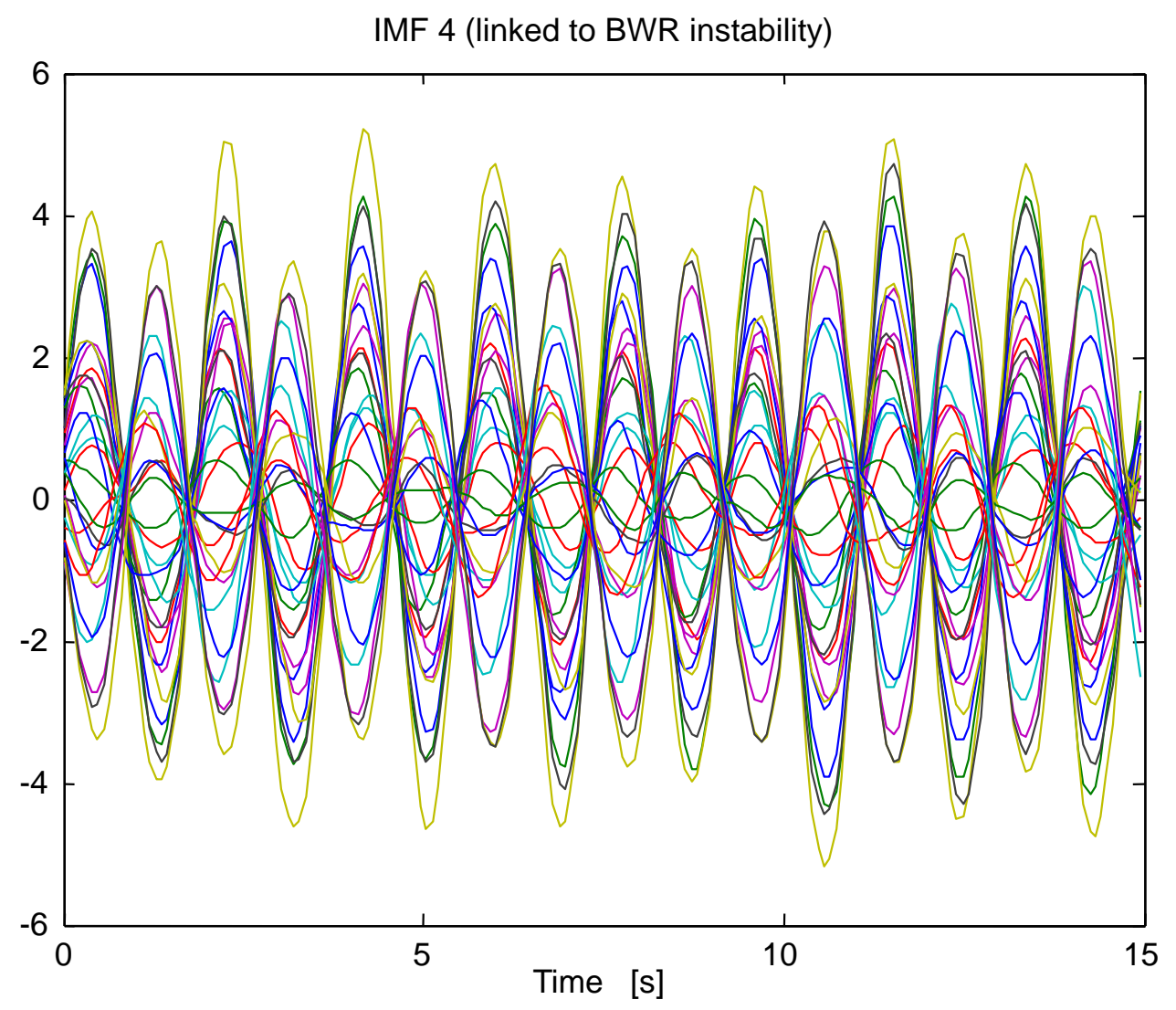

Figure 67. NA-MEMD applied to a short time segment of the Case 9 Cycle 14 of the Ringhals stability benchmark 


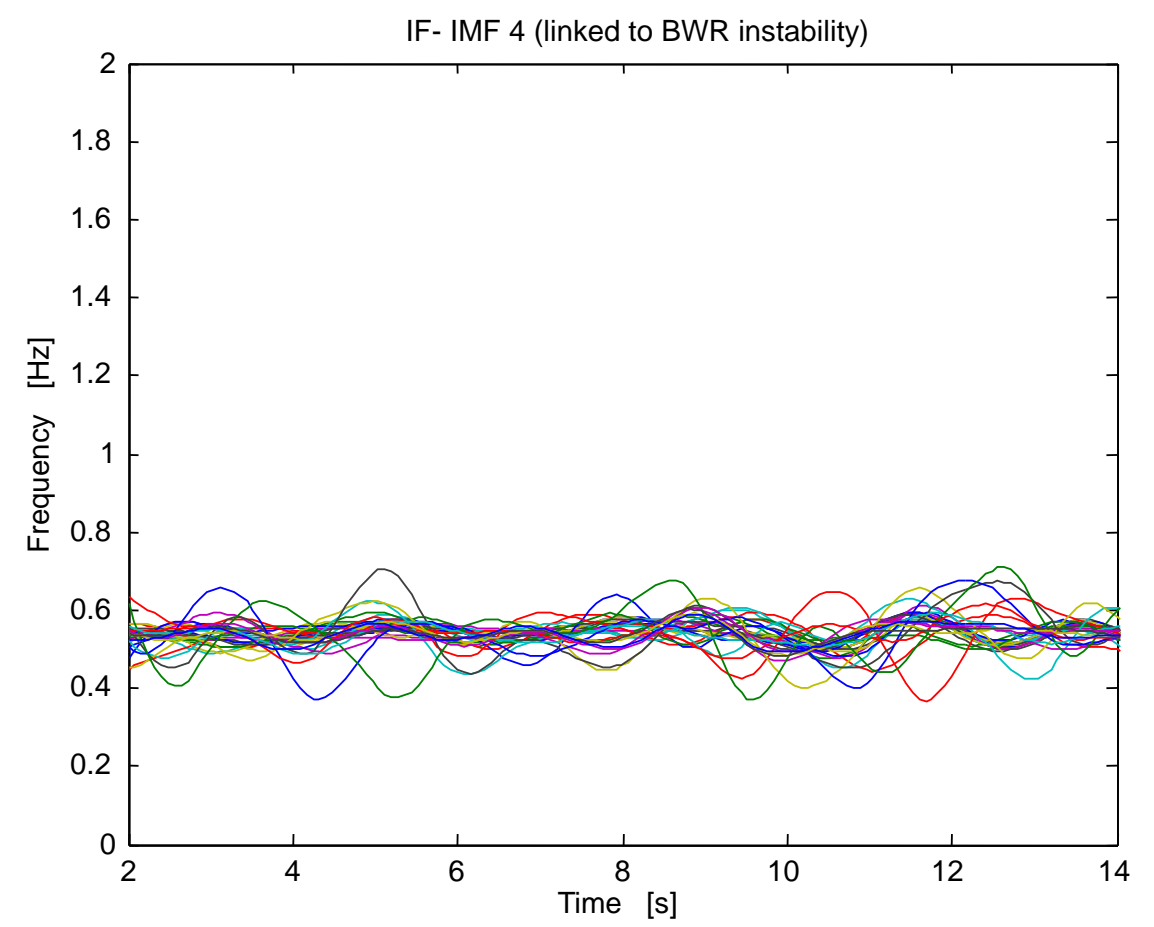

Figure 68. Multivariate instantaneous frequency IF (IF 4) linked to BWR instability oscillating around the region of interest $(0.5 \mathrm{~Hz})$.

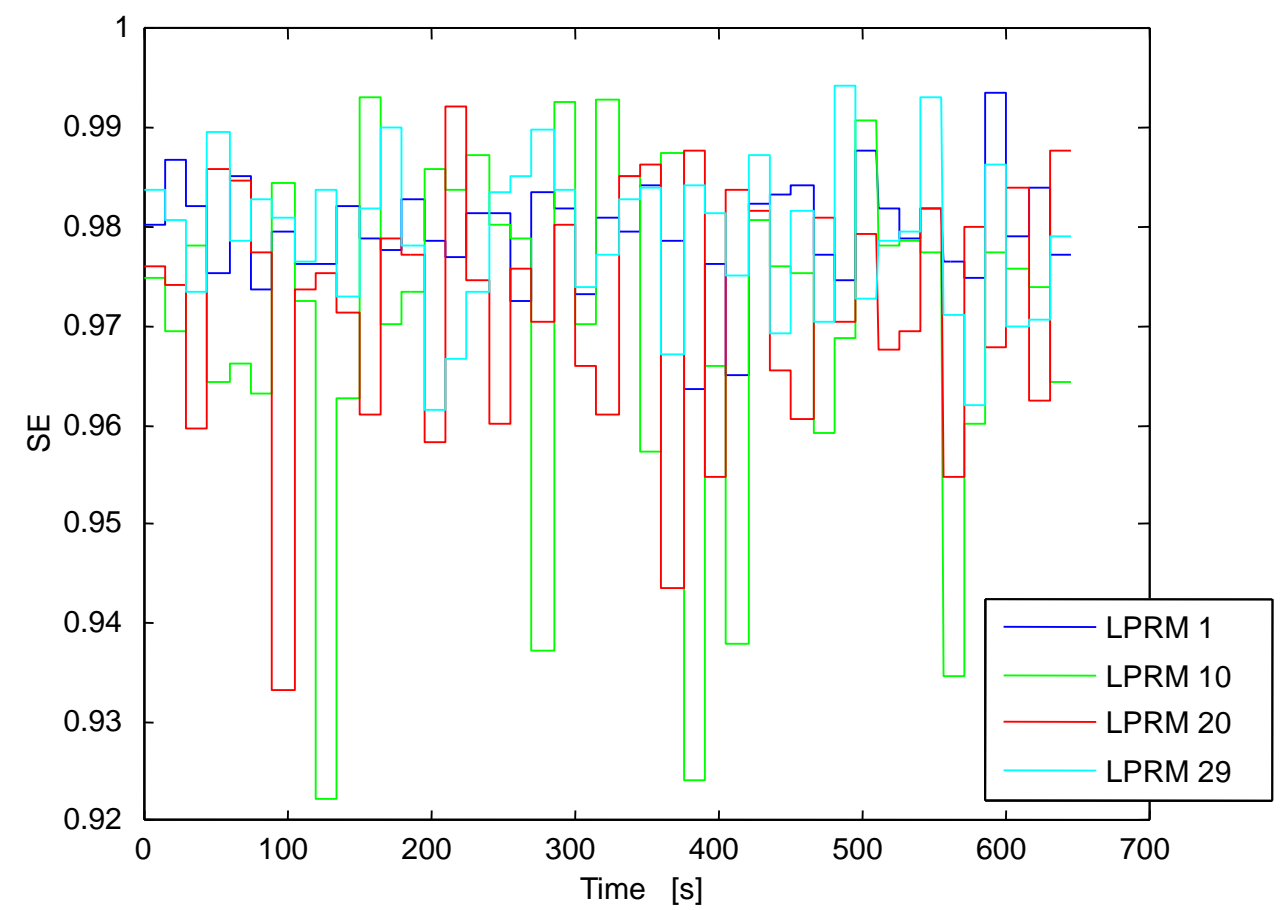

Figure 69. Local SE estimate along time for the selected 4 LPRM sample. All of the SE estimates exceed the stability threshold (located at $\mathrm{SE}=0.8$ ). 
Figure 70 shows the DR estimates along time for the chosen LPRMs, the DR estimates were high, clearly indicating the beginning of an unstable event, but they did not exceed the stability threshold to trigger BWR protection mechanisms.

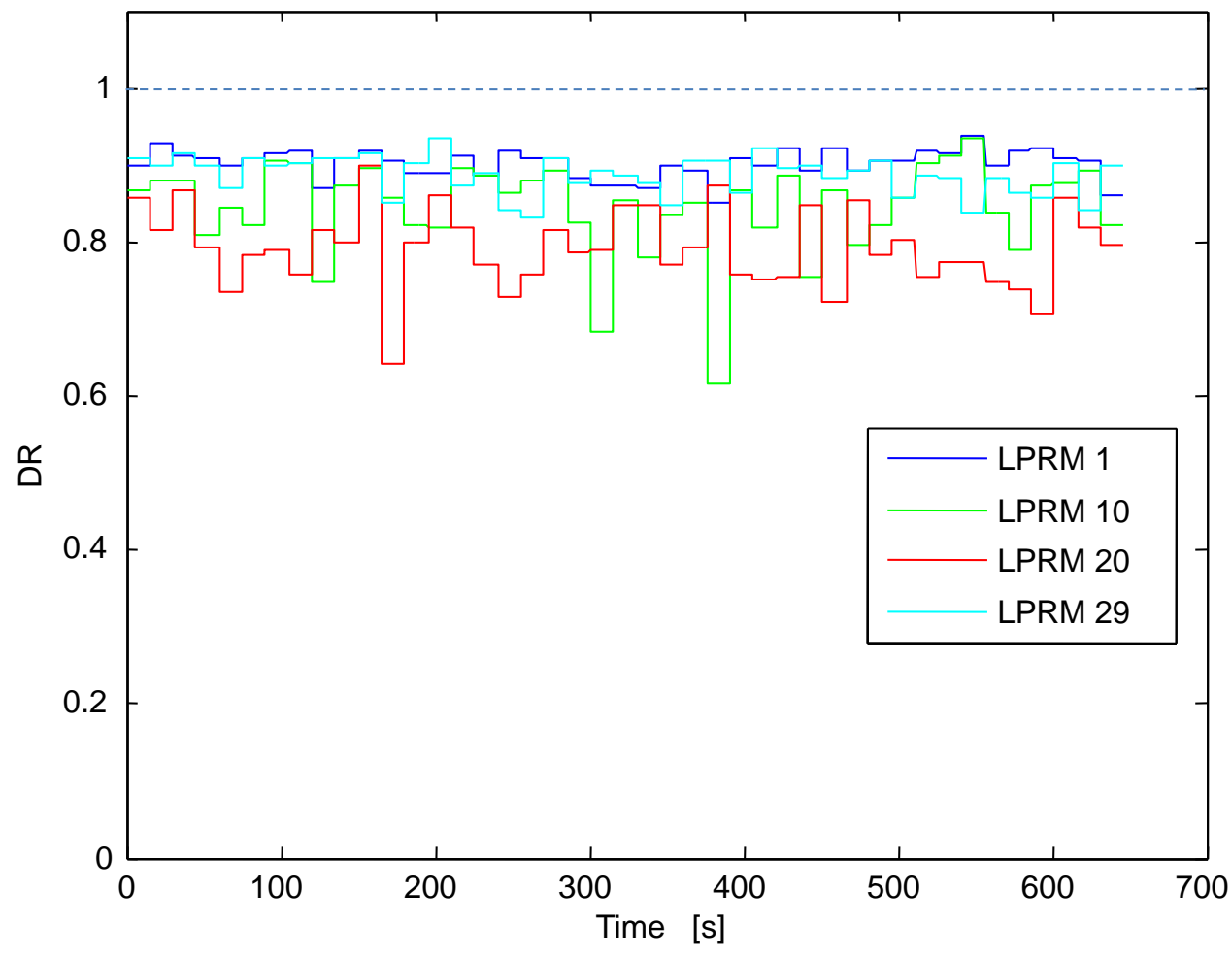

Figure 70. Local DR estimate along time for the selected 4 LPRM sample. The threshold DR bar is located at the same locus as before.

Table 10 shows the SE, DR and oscillation frequency of the entire Ringhals Case 9. Again, the computer parameters in Table 10 are similar (less than $10 \%$ of difference) with the estimates shown previously in Table 7 when this case was analyzed (in an univariate way) through Methodology 1. We highlight that the NA-MEMD capabilities to compensate for mode mixing with only one realization of the algorithm whereas the improved CEEMDAN required a total of $\mathrm{I}=100$ (the size of the ensemble) realizations of the default EMD algorithm to compensate for it. Thus, the NA-MEMD excels in computation time and the SE and DR estimates Methodology 2 provides were slightly the same as those given by stability methodology 1 .

Table 10. Average and standard deviations values for the SE, the DR and the oscillation frequency $\left(f_{0}\right)$ linked to instability of the Ringhals benchmark stability Case 9 Cycle 14 studied via Methodology 2 based on NA-MEMD.

\begin{tabular}{ccccccc}
\hline Detectors & Mean SE & Std SE & Mean DR & Std DR & Mean f & Std f \\
\hline LPRM 1 & 0.9792 & 0.0064 & 0.9033 & 0.0170 & 0.5271 & 0.0223 \\
LPRM 3 & 0.9779 & 0.0062 & 0.9006 & 0.0158 & 0.5284 & 0.0215 \\
LPRM 5 & 0.9786 & 0.0089 & 0.8997 & 0.0194 & 0.5286 & 0.0209 \\
\hline
\end{tabular}




\begin{tabular}{|c|c|c|c|c|c|c|}
\hline LPRM 7 & 0.9793 & 0.0091 & 0.8841 & 0.0315 & 0.5251 & 0.0235 \\
\hline LPRM 9 & 0.9791 & 0.0057 & 0.9003 & 0.0207 & 0.5244 & 0.0268 \\
\hline LPRM 11 & 0.9792 & 0.0065 & 0.9007 & 0.0206 & 0.5270 & 0.0237 \\
\hline LPRM 13 & 0.9762 & 0.0087 & 0.9005 & 0.0196 & 0.5306 & 0.0182 \\
\hline LPRM 15 & 0.9790 & 0.0070 & 0.8977 & 0.0196 & 0.5256 & 0.0247 \\
\hline LPRM 17 & 0.9808 & 0.0083 & 0.8910 & 0.0237 & 0.5239 & 0.0260 \\
\hline LPRM 19 & 0.9708 & 0.0192 & 0.8468 & 0.0594 & 0.5373 & 0.0073 \\
\hline LPRM 21 & 0.9750 & 0.0121 & 0.8682 & 0.0421 & 0.5337 & 0.0141 \\
\hline LPRM 23 & 0.9802 & 0.0086 & 0.8894 & 0.0266 & 0.5307 & 0.0193 \\
\hline LPRM 25 & 0.9789 & 0.0074 & 0.8930 & 0.0230 & 0.5286 & 0.0221 \\
\hline LPRM 27 & 0.9776 & 0.0141 & 0.8881 & 0.0360 & 0.5314 & 0.0189 \\
\hline LPRM 29 & 0.9728 & 0.0229 & 0.8402 & 0.0591 & 0.5346 & 0.0181 \\
\hline LPRM 31 & 0.9635 & 0.0363 & 0.8150 & 0.0792 & 0.5381 & 0.0140 \\
\hline LPRM 33 & 0.9648 & 0.0187 & 0.7227 & 0.1068 & 0.5318 & 0.0224 \\
\hline LPRM 35 & 0.9681 & 0.0213 & 0.7680 & 0.0865 & 0.5308 & 0.0196 \\
\hline LPRM 37 & 0.9769 & 0.0123 & 0.8573 & 0.0393 & 0.5304 & 0.0166 \\
\hline LPRM 39 & 0.9731 & 0.0113 & 0.7923 & 0.0483 & 0.5310 & 0.0228 \\
\hline LPRM 41 & 0.9544 & 0.0306 & 0.6935 & 0.1651 & 0.5312 & 0.0324 \\
\hline LPRM 43 & 0.9600 & 0.0295 & 0.7080 & 0.1310 & 0.5368 & 0.0316 \\
\hline LPRM 45 & 0.9471 & 0.0349 & 0.5754 & 0.2262 & 0.5408 & 0.0456 \\
\hline LPRM 47 & 0.9782 & 0.0073 & 0.8511 & 0.0418 & 0.5279 & 0.0199 \\
\hline LPRM 49 & 0.9796 & 0.0074 & 0.8805 & 0.0255 & 0.5310 & 0.0162 \\
\hline LPRM 51 & 0.9803 & 0.0065 & 0.8992 & 0.0179 & 0.5299 & 0.0169 \\
\hline LPRM 53 & 0.9786 & 0.0055 & 0.8999 & 0.0149 & 0.5271 & 0.0194 \\
\hline LPRM 55 & 0.9813 & 0.0043 & 0.8970 & 0.0195 & 0.5293 & 0.0185 \\
\hline LPRM 57 & 0.9802 & 0.0068 & 0.8868 & 0.0263 & 0.5274 & 0.0204 \\
\hline LPRM 59 & 0.9730 & 0.0329 & 0.8719 & 0.1111 & 0.5254 & 0.0202 \\
\hline LPRM 61 & 0.9698 & 0.0446 & 0.8734 & 0.1171 & 0.5272 & 0.0189 \\
\hline LPRM 63 & 0.9680 & 0.0529 & 0.8790 & 0.0999 & 0.5276 & 0.0187 \\
\hline LPRM 65 & 0.9646 & 0.0669 & 0.8737 & 0.1080 & 0.5265 & 0.0186 \\
\hline LPRM 67 & 0.9685 & 0.0489 & 0.8734 & 0.0948 & 0.5269 & 0.0189 \\
\hline LPRM 69 & 0.9717 & 0.0416 & 0.8735 & 0.1010 & 0.5239 & 0.0228 \\
\hline LPRM 71 & 0.9752 & 0.0218 & 0.8722 & 0.1014 & 0.5275 & 0.0177 \\
\hline
\end{tabular}

\section{Discussions}

Some important final remarks can be done regarding our proposal and recent researches about BWR stability:

The common mechanism for BWR instability is the density wave (DW) oscillations effect (March-Leuba and Blakeman, 1991). A decrease in coolant flow increases the void fraction for a given reactor power. A high wave propagation velocity of voids (wave void) is then formed and accompanied by a high wave propagation velocity of pressure (wave pressure). Since an increase in pressure drop decreases the flow due to increased resistance to flow, a feedback loop results between inlet flow and pressure drop, which may lead to oscillations 
in time. In addition, as the void fraction is increased as described above, the associated decrease in moderator density induces a negative reactivity feedback. This causes the power to decrease, which reduces the void fraction and fuel temperature and allows the power to build up again. As a result, self-sustained power oscillations may appear, depending on the operation conditions.

According with (Muñoz-Cobo et al., 2016) the in-phase instabilities are driven by the interaction between the DWO mechanism and its coupling via the void reactivity feedback with the core neutron population. On the other hand, an in-phase instability implies growing neutron oscillations that are dominated by the fundamental neutronic mode. Regarding to the first azimuthal neutronic mode may also be unstable and growing, but its contribution to the total neutron population is relatively insignificant (Dokhane et al., 2007).

The mechanism of density wave oscillations for two-phase flow has recently received great attention, remaining as an important issue of scientific and technological interest (e.g. Muñoz-Cobo et al., 2016; Demeshko et al., 2015; Paul and Singh, 2014; Paul and Singh, 2017; Vinai et al., 2014; Paruya et al., 2016; Pandey and Singh, 2017; Marcel et al., 2017). However, the core stability is due to fluctuations in coolant flow and power generation process coupled via nuclear feedback where the non-linear nature has been a challenge for the development of stability monitors. Therefore the methodology presented in this work constitutes a significant and novel advance towards the development of stability monitors able to predict linear and nonlinear effects, as well as the transition between them.

Experiments on natural circulation BWR stability show that changing the fuel rods diameter affect to the stability performance of the system (Marcel et al., 2017). These authors clearly observed that at least two oscillatory modes exists in the system, one of them is the so-called reactor mode related to density waves travelling through the core, which is amplified by increasing the void reactivity feedback coefficient. Therefore, the methods based on SE presented in this work, are applicable to existing and advanced reactors of type BWR, and any two-phase flow system as well as characterization of stability limits (Pandey and Singh, 2017). A recent work showed that the stability of a BWR reactor was applied to assessment of optimum Fuel Reload Patterns for a BWR (Castillo-Durán et al., 2016).

The methodology 1, developed in this work, is limited to the cases of neutron signal analysis of an APRM or LPRM where the instability in-phase can be detected like in a NPP as Laguna Verde which characteristic is its size (smaller compare to Forsmark and Ringhals) and where this kind of instability phenomena is expected. Regarding to methodology 2, it can be applied to both phase in-phase and out-of-phase instabilities. Given that the stability phenomena in BWR is a complex phenomenon in a heterogeneous two-phase flow system, where void propagation waves (propagation of the gas phase in the liquid phase) and pressure propagation waves (both in gas phase and liquid phase) generate the DW oscillation mechanics, then is preferable to implement an oscillation detector based on methodology 


\subsection{Chapter 5 Conclusions}

In this section two non-linear stability monitor methodologies based on noise assisted empirical mode decomposition methods (NA-EMDm) were proposed to analyze unstable BWR signals that stemmed from the Ringhals, Forsmark stability benchmarks and the Laguna Verde instability event, with the goal in mind of estimating the Shannon Entropy of those signals to measure their uncertainty and thus assess BWR stability through such novel measure. Also, the SE estimates were compared with Decay Ratio results computed via previous methods based on EMD variants. The proposed stability methodologies are rooted in noise assisted empirical mode decomposition algorithms, which are techniques that decompose non stationary signals that stem from non-linear sources in an adaptive (datadriven) way to grant a physically meaningful decomposition of data, the data (the LRPM or APRM signals are split first in segments of $15 \mathrm{~s}$ ) is decomposed into intrinsic mode functions (or simply modes), via the Hilbert transform it is possible to compute the instantaneous frequencies of the extracted modes to track the mode linked to BWR instability (whose IF is strongly concentrated around $0.5 \mathrm{~Hz}$, the region of interest for BWR unstable events). Later, once the IMF (IMFs in the multidimensional case) of interest has been detected, the SE of this particular IMF is computed to assess the BWR stability of that particular $15 \mathrm{~s}$ signal segment that was analyzed via any of our stability methodologies. The major findings of our BWR stability studies are resumed in the following:

a) regarding Methodology 1 based on the iCEEMDAN (univariate signal analysis)

- $\quad$ Case 4 of the Forsmark stability benchmark

The estimated averaged values for the DR are in perfect agreement with those estimated by the different methodologies presented in [3]. The DR estimates indicate the beginning of an incipient instability event whereas the SE estimates indicate a fully developed instability event in the BWR core.

- $\quad$ Case 9 Cycle 14 of the Ringhals stability benchmark

The high SE estimated values, clearly indicate again a fully developed unstable behavior of this case. Thus, the studied BWR floor 2 is unstable. The high DR estimates (but still not above the locus DR = 1) although high and depicting that there is something unusual going on in the BWR core but not high enough to trigger BWR protection mechanisms.

- The Laguna Verde instability event

Prior the first $300 \mathrm{~s}$ of the signal, the DR oscillates between stability and instability. But, it is hard to infer the dominant DR value due to its strong discontinuous jumps between stability and instability. However, after the $300 \mathrm{~s}$ mark, the DR is high and greater that its threshold value $(D R=1)$ and remains as such (and oscillating around 1.1) throughout the rest of the simulation. Thus, the DR indicates unstable BWR behavior but only after the $300 \mathrm{~s}$ mark. The SE estimate is highly more consistent than the DR prior the $300 \mathrm{~s}$ mark, because the 
SE clearly indicates unstable behavior (whereas the DR is unable to differentiate between the two) and after the $300 \mathrm{~s}$ mark, the SE slightly oscillates around 1 (and not in a dramatic way as the DR does). Nevertheless, the SE always indicates unstable BWR behavior, long before the DR is able to detect it. Thus, the SE is capable of indicating unstable behavior prior any further growth in power of the unstable oscillation within the core whereas the DR is only able to detect instability (without bias) once the unstable oscillation is fully sustained and powerful enough to damage the core.

b) regarding Methodology 2 based on the NA-MEMD (multivariate signal analysis)

- Multivariate analysis of the Forsmark stability benchmark (based on a sample of 4 LPRMs)

Overall the BWR is unstable because of the high SE estimates along time, in spite of 4 segments that had an SE below the stability threshold $(\mathrm{SE}<0.8)$. Thus, again from the very beginning of the simulation, the SE is able to detect an unusual BWR unstable behavior. The DR in the multivariate case prior the $150 \mathrm{~s}$ mark is apparently stable and after this $150 \mathrm{~s}$ mark, it fluctuates around 0.75 , the DR estimate is high but not high enough to be a nuisance for BWR operation.

- Multivariate analysis of the Forsmark stability benchmark (based on a sample of 4 LPRMs):

The SE estimates along time were high (beyond the SE stability threshold located at $\mathrm{SE}=0.8$ ) throughout the time span of the simulation for the 4 chosen LPRMs, thus the BWR is clearly unstable whereas, the DR estimates were high, clearly indicating the beginning of an unstable event, but they did not exceed the stability threshold to trigger the BWR protection mechanisms.

According to our simulations it is naive to assume to infer information associated to BWR dynamics through one linear parameter alone such as the DR, because in most of our simulations, the DR only rises above its stability threshold (DR above 1) once the unstable oscillation has grown enough in power to damage the core (according to the stability analysis of the LV signal). Thus, it is necessary to propose another non-linear stability indicator (to replace the DR or to accompany it) to assess BWR stability, and the SE might be a suitable candidate to fulfill that role via our simple SE estimator or another more elaborate one that will be studied in future works.

To select which stability methodology (between 1 and 2) is the most adequate to analyze BWR signals, is still not known and further stability cases must be studied in detail to decide which type of analysis works better; whether a univariate one or a mutlivariate one. Nevertheless, the SE (and DR) estimates extracted through these decomposition methods were similar (within the $10 \%$ of difference). These noise assisted techniques have one cumbersome inconvenient and a difficult one to overcome. For instance, how to properly select the iCEEMDAN parameters $I$ (the size of the ensemble of realizations of the EMD that this noise assisted method requires) and $\varepsilon_{0}$ (the standard deviation of the added assisted noise)? Nobody knows that answer yet in the EMD literature, thus further studies 
are required to infer these two parameters. A similar question arises with the NA-MEMD, how many independent channels of noise are required in the decomposition scheme to mitigate the mode-mixing problem?, again, another question that has not been addressed in the specialized literature. However, once these questions are answered, then, our stability methodologies might be fully adaptive to be implemented in a real stability monitor and well adapted to decompose non stationary non linear data. 


\section{Chapter 6}

\section{BWR Stability Indicator 2: The Sample Entropy}

In this chapter, we explore the Sample Entropy (SampEn) linked to the noise-assisted multivariate empirical mode decomposition (NA-MEMD) to infer whether BWR signals are stable or not. The SampEn (Richman and Moorman, 2000) is a measure that provides an index of signal complexity or irregularity of a time series. In this way, the SampEn might work as a possible nonlinear BWR stability indicator. SampEn was in principle developed almost exclusively to study physiological time series, over time, its utility expanded to other domains, such as: for daily weather temperature to measure climate complexity ( $\mathrm{Li}$ et al., 2006) and for the measure the complexity of the dynamic reconfiguration of the brain to infer its association with normal aging (Jia et al., 2017).

To properly estimate the SampEn from real BWR signals, the NA-MEMD was explored. The methodology we introduce here is based on the NA-MEMD and the HHT to compute an indicator linked to BWR density wave instability, such novel indicator is the Sample Entropy.

\subsection{The Sample Entropy}

The procedure for computing the sample entropy SampEn was introduced by Richman and Moorman (Richman and Moorman, 2000). In here, we grant a brief summary of their findings. SampEn is a measure of complexity. Let $x=\left[\mathrm{x}_{1}, \mathrm{x}_{2}, \ldots, \mathrm{x}_{N}\right]$ be a time series of length $N$. To compute the complexity of this time series via SampEn, follow the next steps:

1. Build a vector $v_{i}$ with $m$ consecutive data points taken from $x$

$$
v_{i}=\left[\mathrm{x}_{i}, \mathrm{x}_{i+1}, \ldots, \mathrm{x}_{i+m-1}\right]
$$

where $m$ is the length of sequences to be compared, also called the embedding dimension. 
2. For each $i$ define:

$$
C_{i}^{m}=\frac{1}{N-m-1} \sum_{j=1, j \neq i}^{N-m} \Theta\left(r-\left\|v_{i}-v_{j}\right\|_{1}\right)
$$

where $i$ varies in the interval $(1 \leq \mathrm{i} \leq \mathrm{N}-m)$. In here, $r$ is the tolerance for accepting matches, $r=s \times \operatorname{std}(x)$ where $s$ is a scaling parameter and $\operatorname{std}(x)$ is the standard deviation of $x . \Theta(\square)$ is the Heaviside function:

$$
\Theta(\mathrm{x})= \begin{cases}0, & x<0 \\ 1, & x \geq 0\end{cases}
$$

and \|\|$_{1}$ is the Chebyshev distance, defined as:

$$
\left\|v_{i}-v_{j}\right\|_{1}=\max \left(\left|x_{i}-x_{j}\right|,\left|x_{i+1}-x_{j+1}\right|, \ldots,\left|x_{i+m-1}-x_{j+m-1}\right|\right)
$$

3. $C_{i}^{m}$ represents the proportion of $v_{j}(i \neq \mathrm{j})$ whose distances to $v_{i}$ are less than $r$. Now, for each $i$ we also define:

$$
C_{i}^{m+1}=\frac{1}{N-m-1} \sum_{j=1, j \neq i}^{N-m} \Theta\left(r-\left\|v_{i}-v_{j}\right\|_{1}\right)
$$

where $C_{i}^{m+1}$ represents the proportion corresponding to the dimension of $m+1$. $C_{i}^{m}$ and $C_{i}^{m+1}$ have the same mold, but embedding vectors in both cases are defined in different spaces. 
4. Average across all embedding vectors, to obtain:

$$
U^{m}=\frac{1}{N-m} \sum_{i=1}^{N-m} C_{i}^{m}
$$

and

$$
U^{m+1}=\frac{1}{N-m} \sum_{i=1}^{N-m} C_{i}^{m+1}
$$

5. The SampEn is computed as

$$
\text { SampEn }=-\ln \left(\frac{U^{m+1}}{U^{m}}\right)
$$

SampEn is the negative natural logarithm of the conditional probability that two sequences similar for $m$ points remain similar at the next point, where self matches are not included in calculating the probability. Thus, a lower value of SampEn indicates more self-similarity (i.e. high order) of the studied time series whereas a higher value of SampEn points to higher complexity of the time series. The calculation of SampEn requires a priori determination of two unknown parameters, $m$ and $r$ (the length of data $N$ is up to the user). The suggested values of $r$ are located in the range of 0.1-0.2 times the standard deviation of the studied signal $x$. In this section, we looked for the $r$ value that grants the global SampEn maximum because this maximum value leads to the correct interpretation of signals complexity (Chon et al., 2009). So, this $r$ of interest is fixed in our simulations at $r=0.1$. The value of $m$ can be computed via the estimation of false nearest neighbor (Kennel et al., 1992). In our particular BWR stability discipline, the $m$ that was found through false nearest neighbors for our signals was most of the time equal to 2 . Therefore, $m$ is fixed at $m=2$ for all of our computations. SampEn is, theoretically speaking, a fraction in the interval $0<\operatorname{SampEn}<\infty$. However, the next two formulas can be used to find the lower bound and the upper bound of SampEn for fixed values of $m$ and $N$. 
The lower bound is computed as:

$$
2[(N-m-1)(N-m)]^{-1}
$$

The upper bound is computed as:

$$
\ln (N-m)+\ln (N-m-1)-\ln (2)
$$

For all of our simulations $m, r$ and $N$ are fixed at $m=2, r=0.1 \times \operatorname{std}(x)$ and $N=300$ data points. Thus the lower bound is practically $\approx 0$ whereas the upper bound is close to 11 . However, the upper bound value was never attained for any simulation.

\subsection{BWR stability methodology based on the SampEn and the NA-MEMD}

The methodology to detect stable or unstable states (this methodology works with raw data. So, there is no need to preprocess the analyzed data) in a core of a BWR, based on the sample entropy and the NA-MEMD, is presented in the next steps:

1. The studied raw signal (APRM or LPRM) obtained from the BWR is segmented in windows of $60 \mathrm{~s}$ of duration.

2. Each segment (of $60 \mathrm{~s}$ of time span) is considered as just an independent channel $(L=1)$ added with $q$ independent channels of white Gaussian noise and decomposed through the NA-MEMD (for all of our computer simulations $q=2$ ).

3. After decomposition, the $q$ channels corresponding to the noise are discarded, giving a set of IMFs corresponding to each decomposed studied segment. 
4. The Hilbert transform of each IMF (i.e. the HHT) is computed to get the instantaneous frequency of each IMF in the studied segment.

5. When tracking these frequencies, it is possible to get the mode (or modes) linked to the density wave oscillation. In this way, only the mode (or modes) associated to BWR instability is considered for further processing whereas the remaining extracted modes are ignored.

6. The SampEn of the tracked mode of interest (IMF) is computed for $N=300$ (the length of the analyzed segment of $60 \mathrm{~s}$ ), $m=2$ and $r=0.1 \times \operatorname{std}($ segment) to attain the global maximum of the SampEn that leads to the correct interpretation of the studied segment complexity (Chon et al., 2009).

7. The mean, median and the variance of the SampEn values are computed and averaged along all the studied segments of $60 \mathrm{~s}$ of the original signal.

A high SampEn estimate (>1) points out high irregularity and high unpredictability of the studied BWR signal (LPRM or APRM) and this high value might indicate that the studied signal could be merely broad band non-coherent noise (BBNCN). This BBNCN, from the BWR point of view is a sign of stable behavior. On the other hand, a low SampEn value $(<0.8)$ points to high predictability and regularity of the studied BWR signal. Such low value might indicate that the studied signal could be a cyclic one. Such waveform is a sign of BWR unstable behavior, due to the presence of a density wave that manifests in the LPRM or APRM signals as a cyclic waveform with a natural frequency that oscillates very close to $0.5 \mathrm{~Hz}$. For forced convection phenomena, in the circulation natural convection the effect of the chimney changes this value (Marcel, 2007; Rohde et al., 2010).

\subsection{Results and discussions}

Now we validate the methodology given in subsection 3.1 with the next data sets, where $f_{s}$ is the sampling frequency of each set:

1. Data set from a typical BWR:

a) 208 steady state condition signals (i.e. stable signals), $f_{s}=5 \mathrm{~Hz}$.

b) 96 unstable signals, $f_{s}=10 \mathrm{~Hz}$.

2. Case 4 of the Forsmark stability benchmark:

a) 1 APRM signal plus $22 \mathrm{LPRM}$ signals, $f_{s}=12.5 \mathrm{~Hz}$. 
3. Case 9 cycle 14 of the Ringhals stability benchmark:

a) 72 LPRM signals (located within 2 levels of the Ringhals reactor, each level has a total of 36 LPRMs, such levels are called 2 and 4), $f_{s}=12.5 \mathrm{~Hz}$.

\subsubsection{Stable signals}

The aim of this test is to observe the demeanor of the BWR in steady-state conditions. The results obtained are presented in Figures 71-77. Figure 71 shows the plot of one APRM stable signal of a typical BWR. Figure 72 shows in red dotted color, a studied segment of $60 \mathrm{~s}$ of duration of the APRM signal with its power spectral density estimate (black-line). Figure 73 shows the first four extracted IMFs of the studied signal, which happens to be noise that is decomposed in a quasi-dyadic fashion by the NE-MEMD. Figure 74 shows the power spectral density of some IMFs.

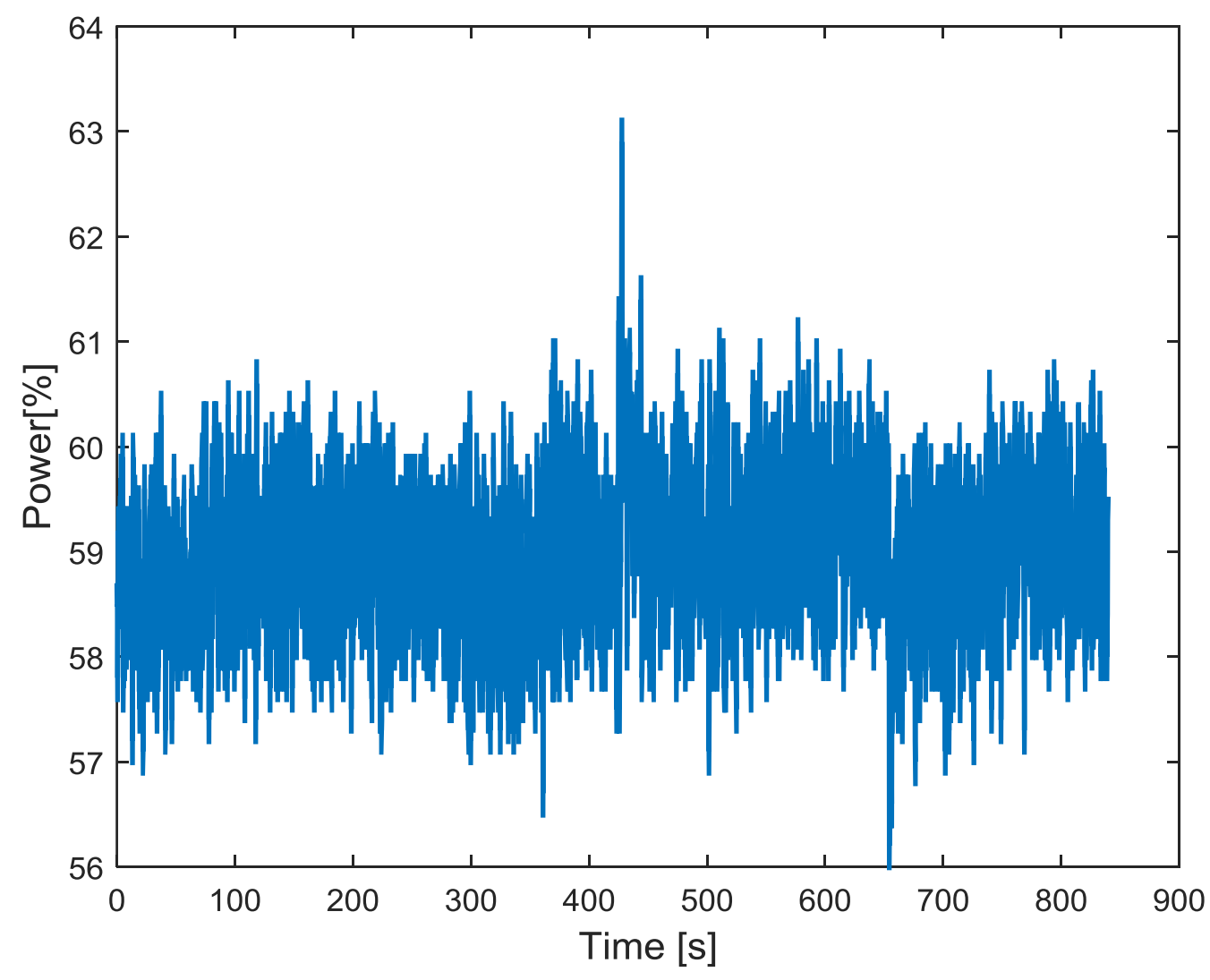

Figure 71. Stable signal from a typical BWR. 

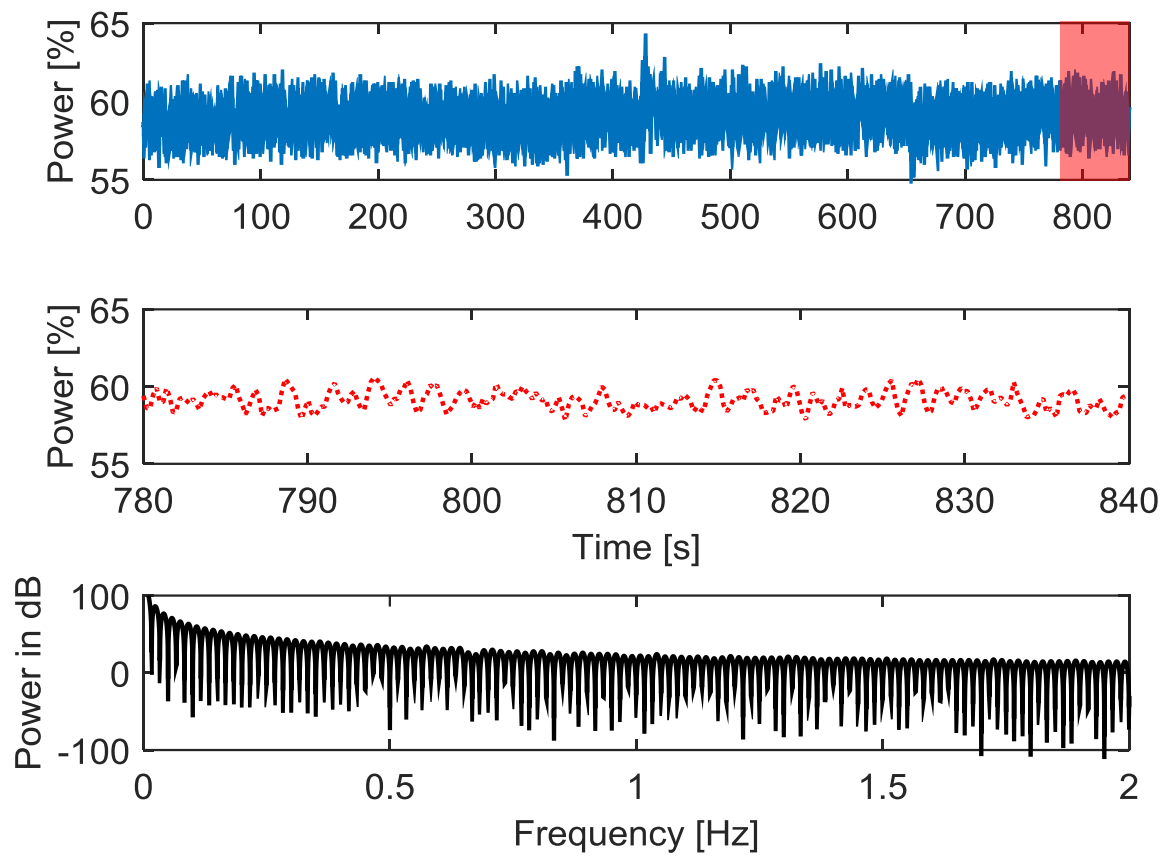

Figure 72. Analyzed segment of the studied APRM signal.
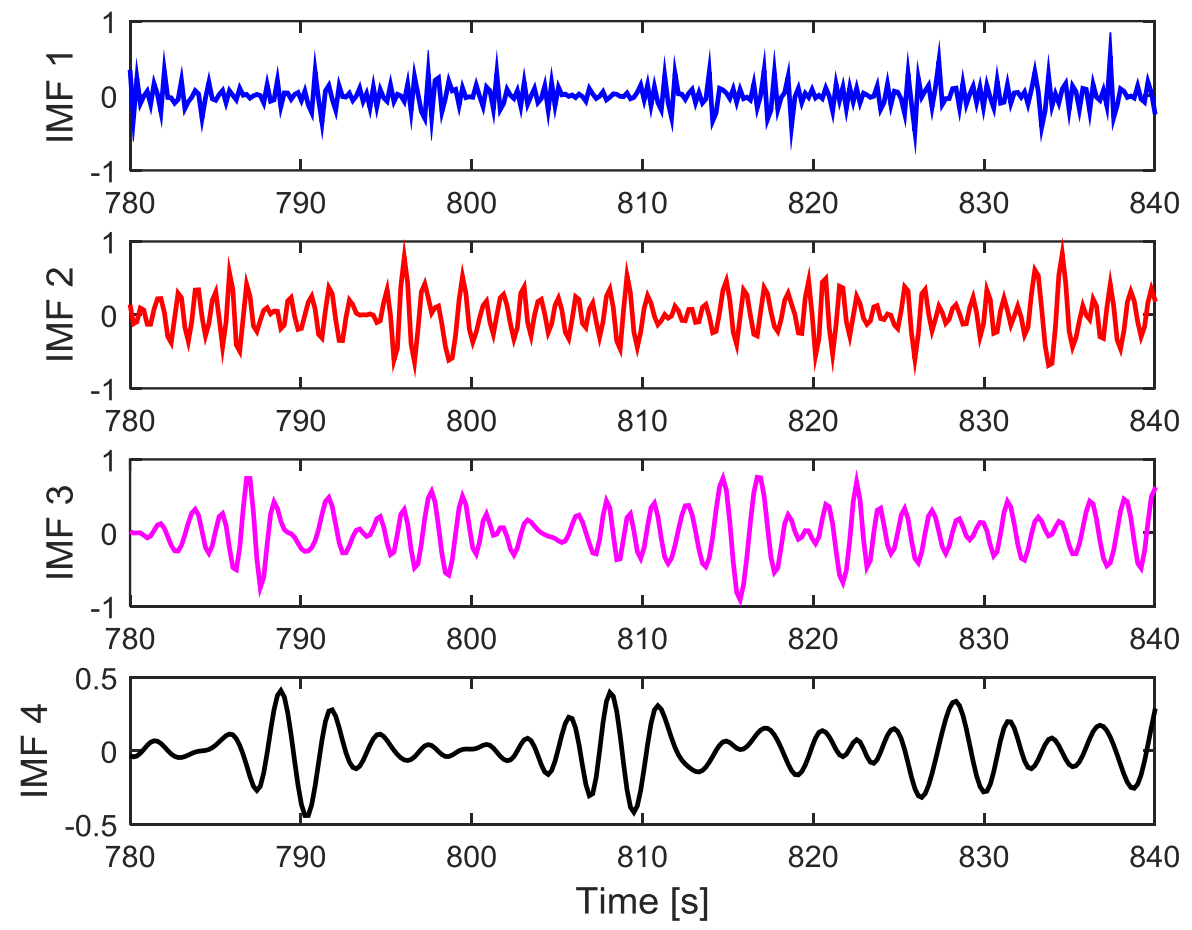

Figure 73. A plot of the first 4 extracted IMFs in the analyzed segment. 


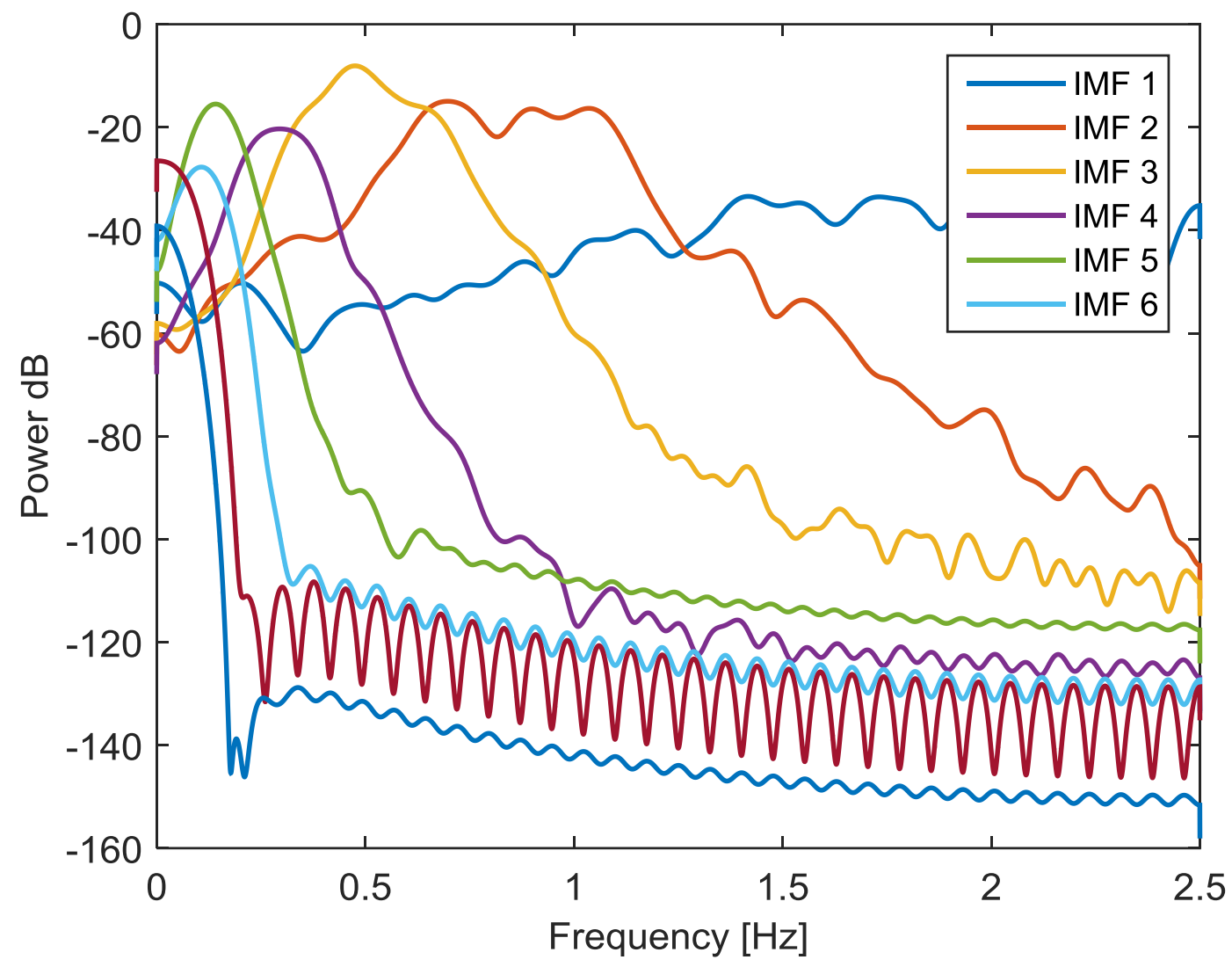

Figure 74. PSD estimate of the first 6 extracted IMFs in the analyzed segment.

Figure 75 shows the tracked IMF (IMF 3) of interest whose instantaneous frequency (IF) oscillates around $0.5 \mathrm{~Hz}$. The computed SampEn of this IMF is 0.9351. Such high SampEn value indicates that the studied signal is broad band non-coherent noise (BBNCN), so the studied segment is stable and far from an unstable behavior. In this stable case, a mode exist whose IF is close to $0.5 \mathrm{~Hz}$. However, the IMF 3 time series looks distant from a cyclic function we look for when an unstable event triggers in the core. All of the IFs were computed through the Hilbert-Huang transform of the extracted IMFs. Figure 76 shows the computed SampEn along time for all of the studied segments of $60 \mathrm{~s}$, most of the SampEn estimates have a value slightly greater than 1 (i.e., the studied signal is stable). Table 11 shows the mean(SampEn), median(SampEn) and std(SampEn) of the studied time series. 

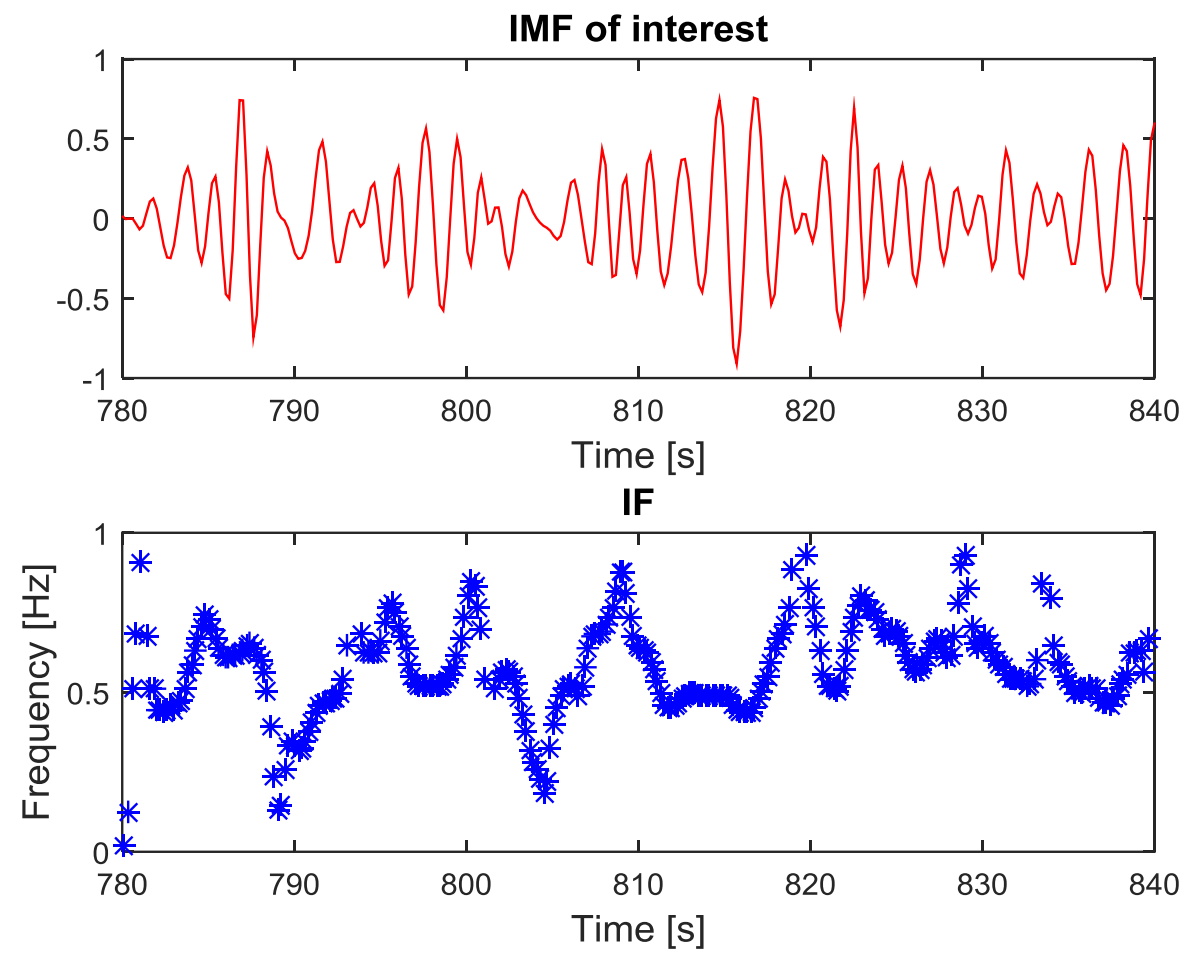

Figure 75. Tracked IMF (IMF 3) of interest and its associated instantaneous frequency (IF) around $0.5 \mathrm{~Hz}$ computed through the HHT.

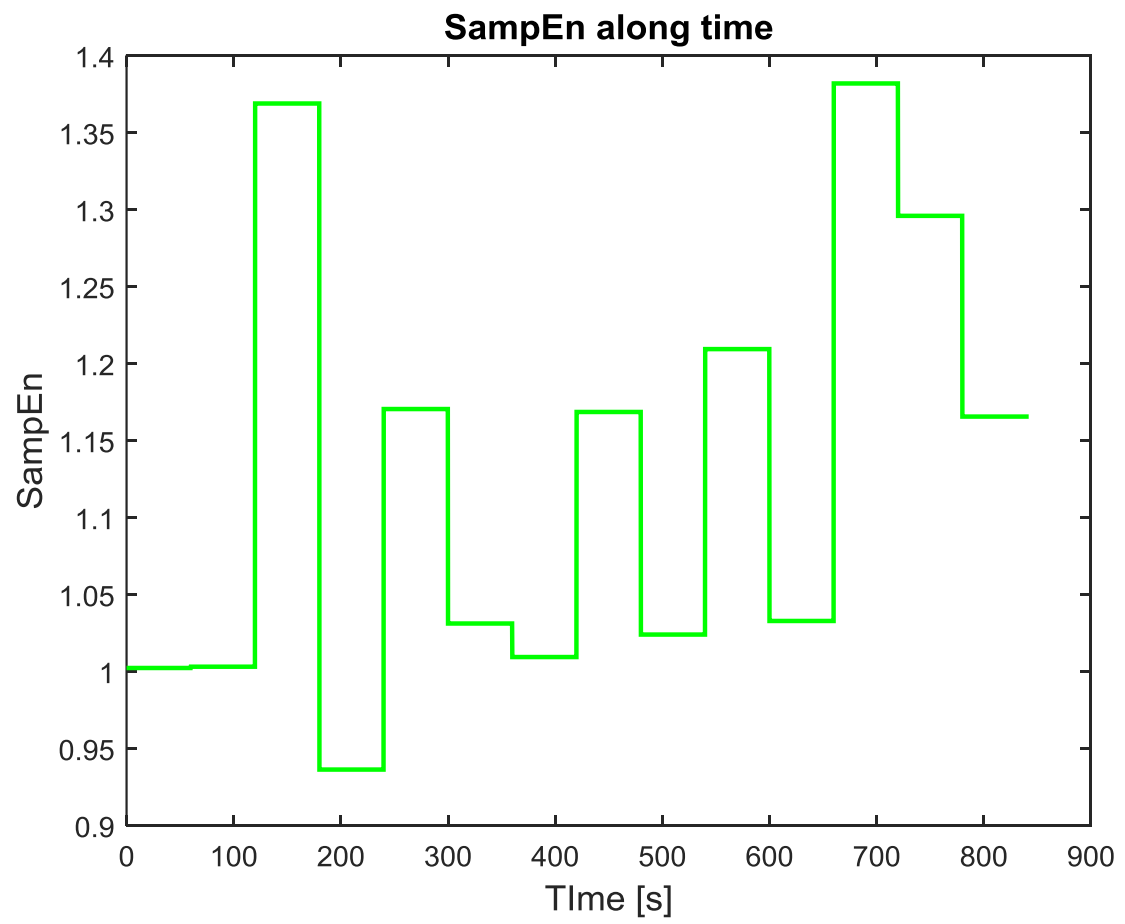

Figure 76. SampEn along time for the studied APRM stable signal. 
Table 11: Mean(SampEn), Median(SampEn) and std(Sampen) of the analyzed APRM.

\begin{tabular}{lccc}
\hline Signal & Mean(SampEn) & Median(SampEn) & Std(SampEn) \\
\hline APRM & 1.1288 & 1.0994 & 0.1398 \\
\hline
\end{tabular}

Figure 77 shows a plot of the computed mean SampEn estimates of the 208 signals (each SampEn estimate is a blue point). A pattern appears, all of the mean SampEn estimates for the studied signals are higher than 1. Thus, the mean SampEn values point to BBNCN. Through SampEn estimation, the proposed stability methodology is able to detect stable signals and classify them in the stable category if their SampEn is greater than 1.

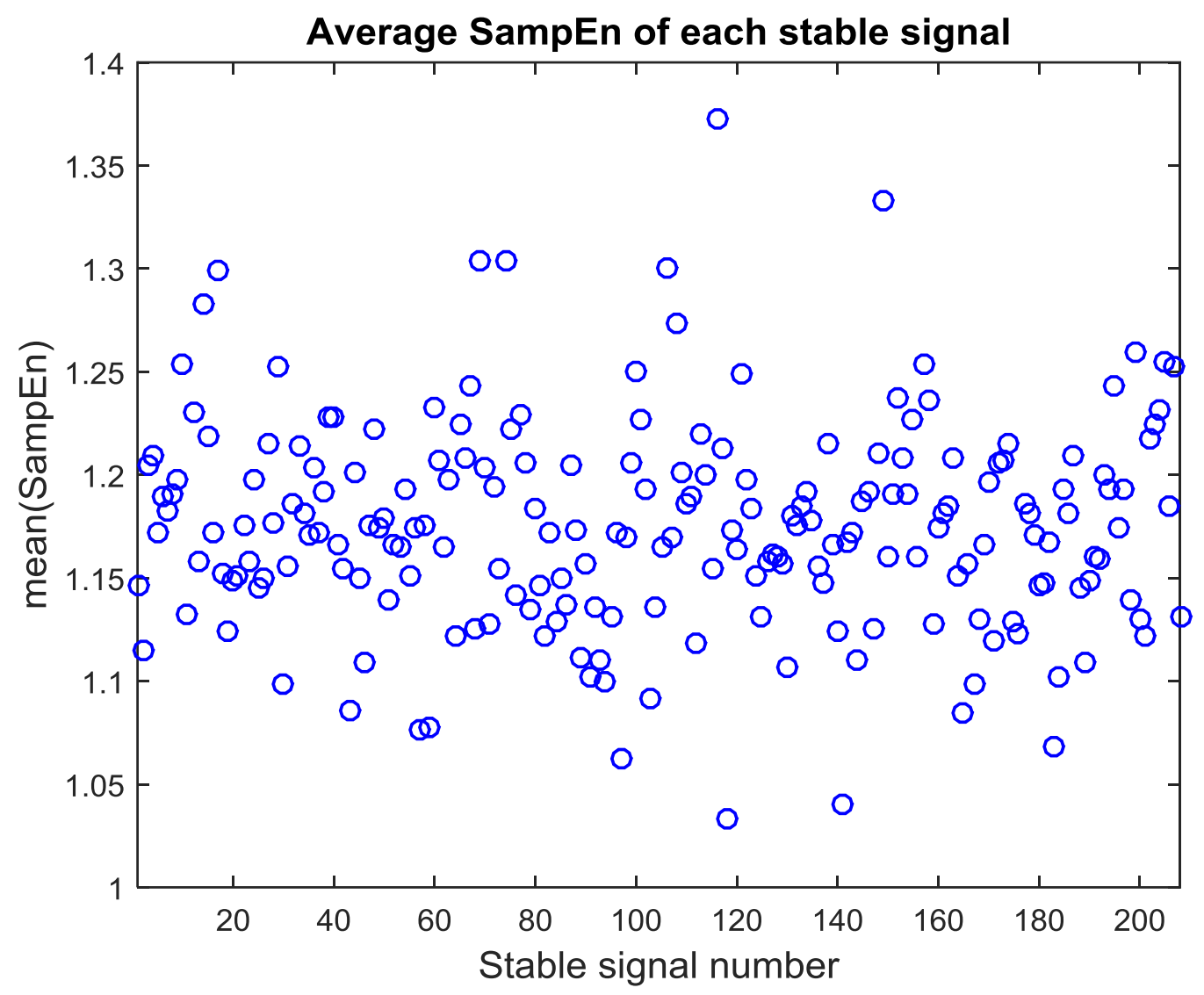

Figure 77. Computed mean(SampEn) of the studied 208 stable signals. 


\subsubsection{Unstable signals}

Now, the studied signals stem from a density wave instability event. This instability case is registered by 96 LPRM signals. For reasons of space, only the analysis of one signal is presented. Figure 78 shows the plot of the studied signal (from LPRM 2), this signal looks like a cyclic function. Figure 79 shows a studied segment of $60 \mathrm{~s}$ (red dotted line) and its PSD estimate (black line), the segment looks like a noisy cyclic function. Figure 80 shows the first 4 extracted IMFs of the studied segment, the first 3 IMFs are linked with acquisition noise, whereas IMF 4 looks like a cyclic function (this is in fact the type of waveform commonly associated with density wave unstable events). Figure 81 shows the PSD estimates of the first 6 IMFs, the energetic content of IMF 4 is highly concentrated around $0.5 \mathrm{~Hz}$, the energetic content of the other IMFs is meaningless next to the energy of IMF 4. The SampEn estimate of IMF 4 (IMF or mode linked to instability, see IF in Figure 82 and PSD estimate in Figure 81) is 0.6181, this estimate is a clear indication of signal regularity and low complexity. The SampEn points to a simple function, in this case a cyclic function that in our context is linked with a density wave type of instability. Thus, the studied segment is unstable. Figure 83 shows the SampEn estimates along time for all of the studied segments of the signal of interest, all estimates are smaller than 1.

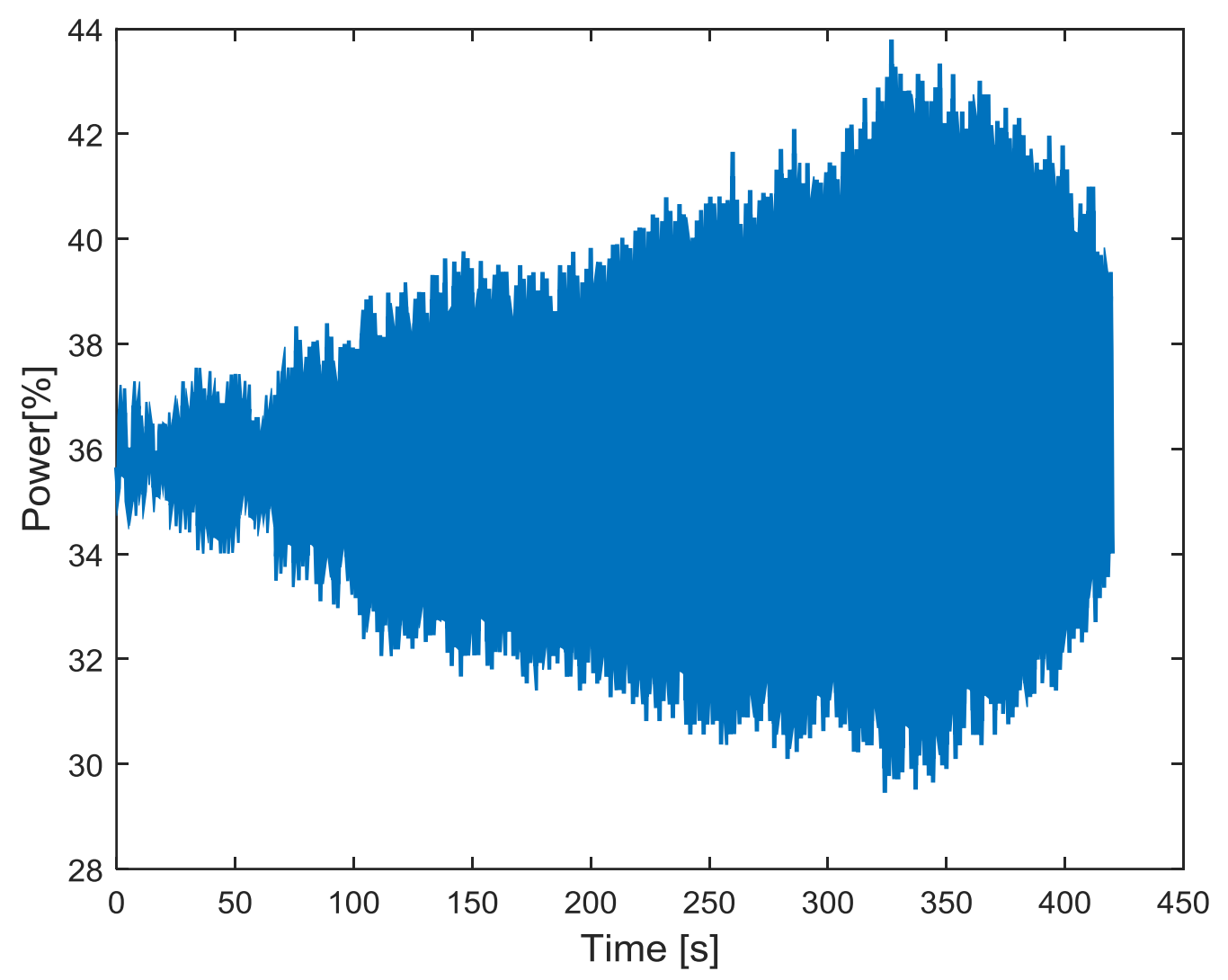

Figure 78. Unstable signal from a typical BWR. 

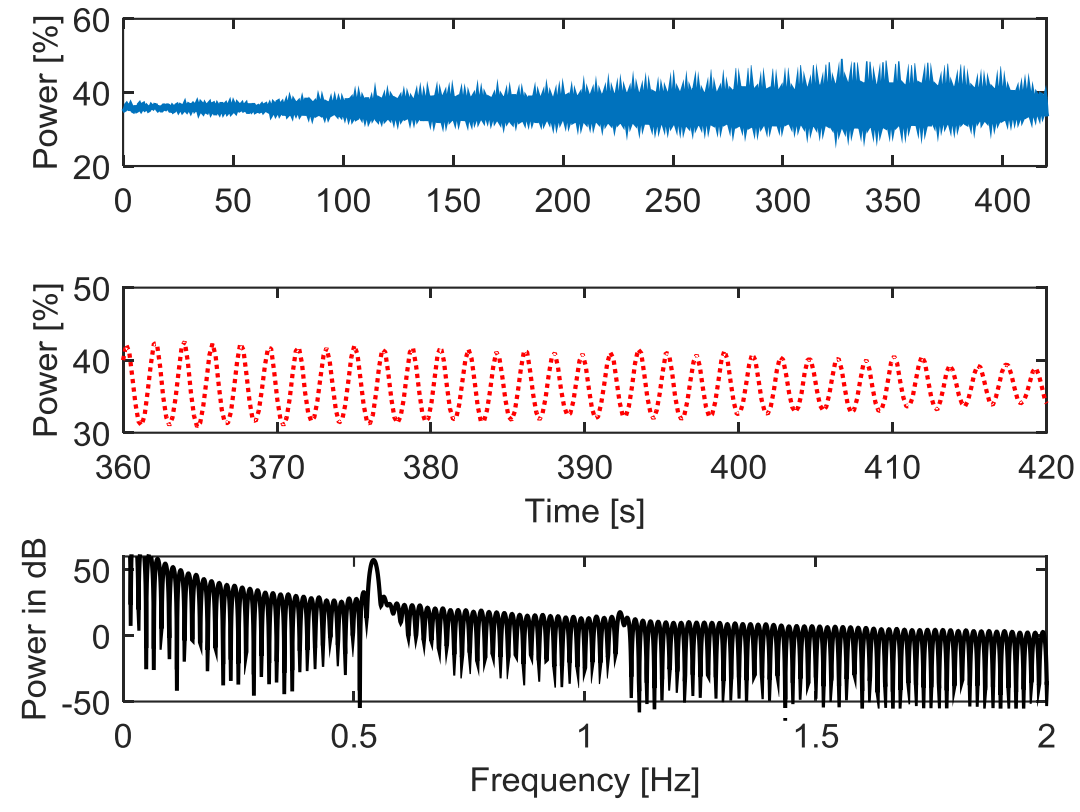

Figure 79. Analyzed segment of the studied LPRM 2 signal.
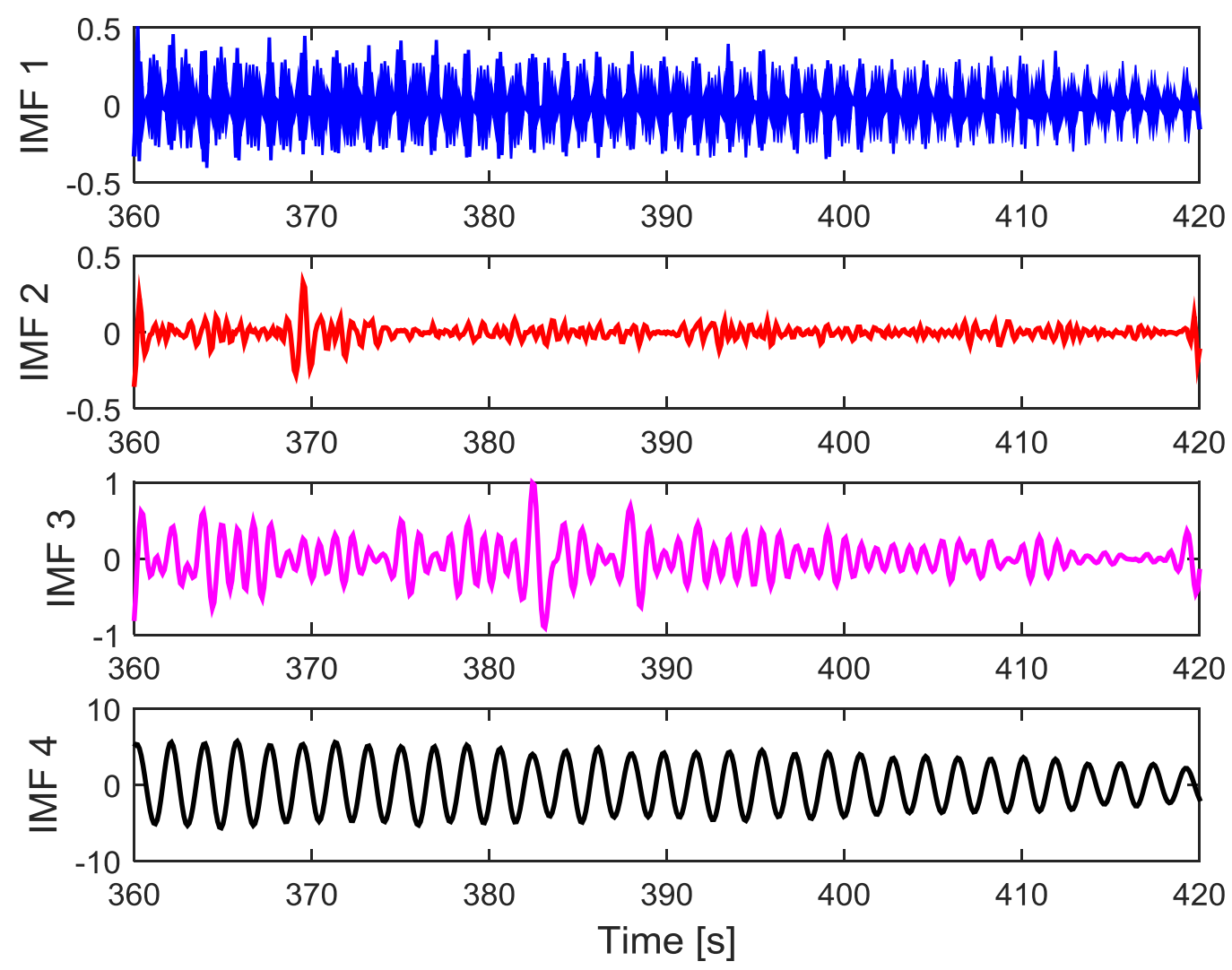

Figure 80. A plot of the first extracted 4 IMFs in the analyzed segment. 


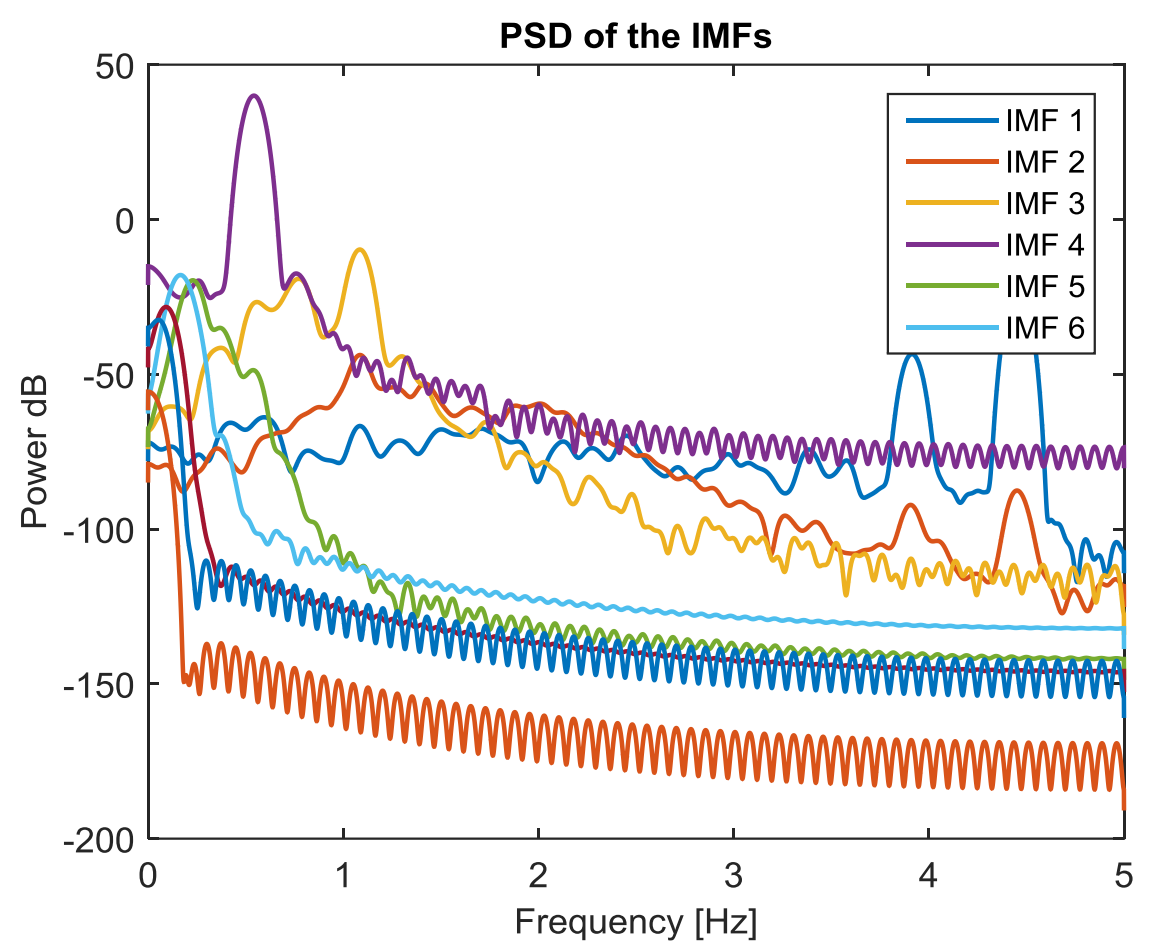

Figure 81. PSD of the first 6 extracted IMFs in the analyzed segment. The PSD of the IMF 4 is highly concentrated around $0.5 \mathrm{~Hz}$.
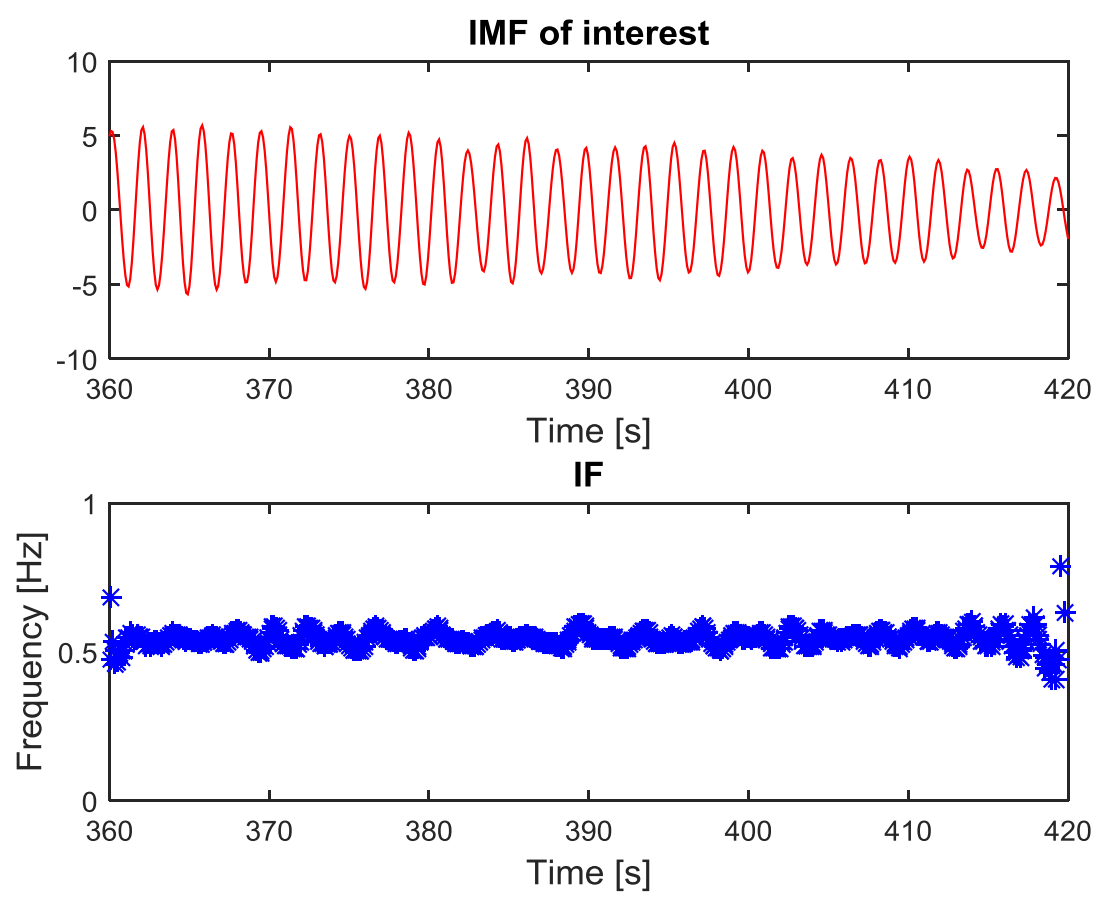

Figure 82. Tracked IMF (IMF 4) of interest linked to BWR instability and its associated instantaneous frequency (IF) around $0.5 \mathrm{~Hz}$ computed through the HHT. 


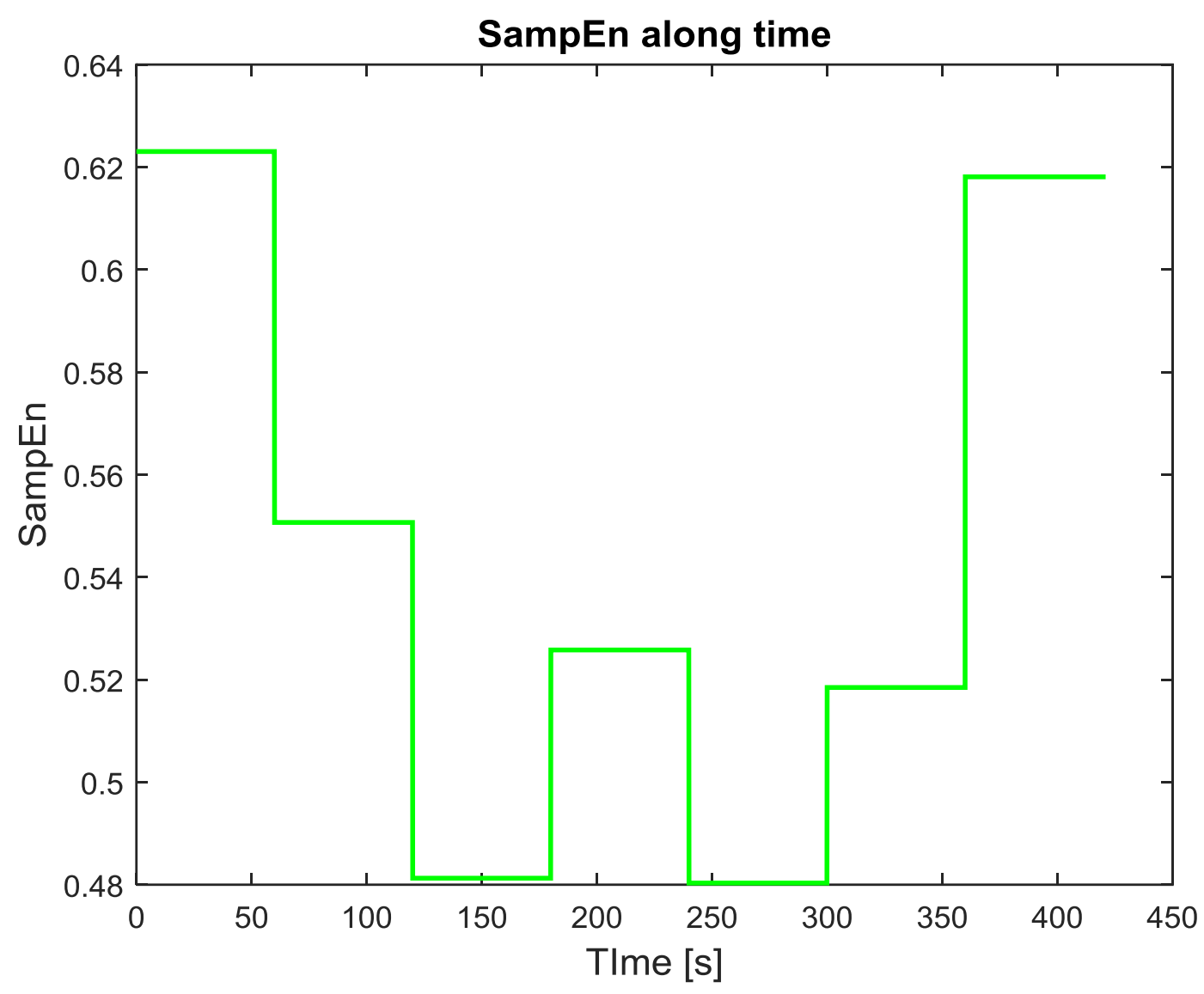

Figure 83. SampEn estimates of the IMFs or modes of interest along time.

Table 12 shows the mean(SampEn), median(SampEn) and std(SampEn) of the time series shown in Figure 83. Now, the tracked IF (IF 4) along time carries physical meaning. Thus, the mean(IF) and std(IF) values of all of the studied segments are now provided in this table.

Table 12: Mean(SampEn), Median(SampEn) and std(Sampen) of the analyzed LPRM 2. Now the mean(IF) and std(IF) are included.

\begin{tabular}{lccccc}
\hline Signal & Mean(SampEn) & Median(SampEn) & Std(SampEn) & $\begin{array}{c}\text { Mean(IF) } \\
{[\mathrm{Hz}]}\end{array}$ & $\begin{array}{c}\text { Std(IF) } \\
{[\mathrm{Hz}]}\end{array}$ \\
\hline LPRM 2 & 0.5425 & 0.5258 & 0.0544 & 0.5374 & 0.0030 \\
\hline
\end{tabular}

Figure 84 shows a plot of the computed mean SampEn estimates of the 96 unstable signals (each SampEn estimate is a red point). A pattern appears, all of the mean SampEn estimates for the studied signals are smaller than 0.8. Thus, the mean SampEn values point to visually regular signals such as cyclic functions. Through SampEn estimation, we are able to build a binary classifier that separates BBNCN signals (linked to stability) from cyclic ones (linked to density wave instability). One core feature of SampEn is that it accommodates for complex non-linear and non-stationary data whereas conventional Decay Ratio estimates 
must assume beforehand that the studied BWR signals behave linearly (an assumption that is false in real life).

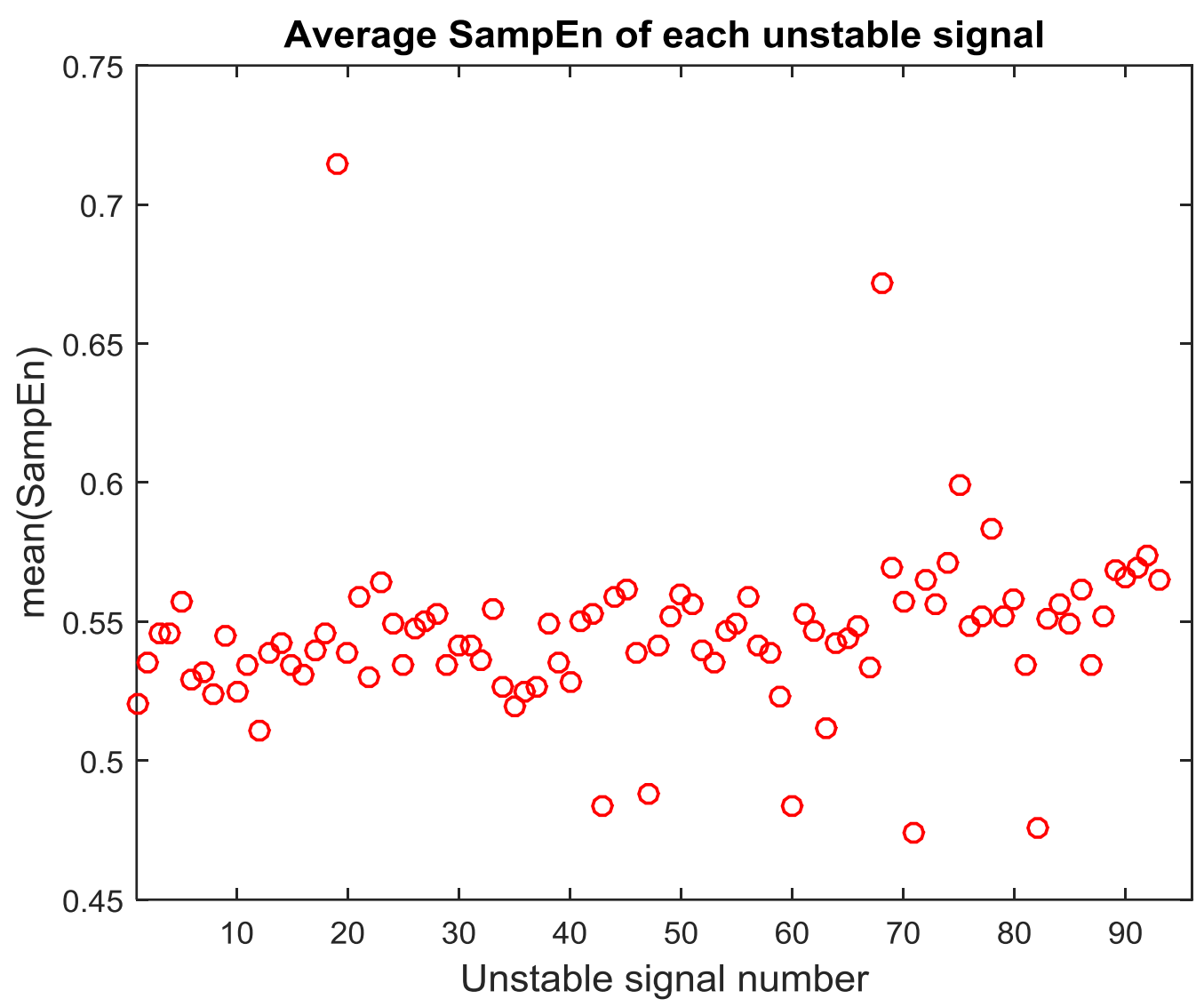

Figure 84. Computed mean(SampEn) of the studied 96 unstable signals.

\subsubsection{Comparison of stable and unstable signals}

To complete the analysis of the stable/unstable signals through SampEn, Figure 85 shows a comparison of the mean SampEn values of the studied sets (stable set of 208 signals plus a set of 96 unstable signals). The computed SampEn mean values of the stable signals fluctuate very close to 1.2 whereas the SampEn mean values of the unstable signals oscillate very close to 0.6. So the SampEn detects with success stable BWR signals from unstable ones via complexity analysis (of course with the aid of the NA-MEMD to denoise these signals and isolate the mode linked to instability). There is a distance of $\approx 0.6$ between the two states (stable from unstable). So, by fixing a threshold value around 0.9, it is possible to differentiate one state from the other properly. Any SampEn estimate of a BWR signal segment higher than 0.9 is stable whereas any lower SampEn value from this threshold is unstable. 


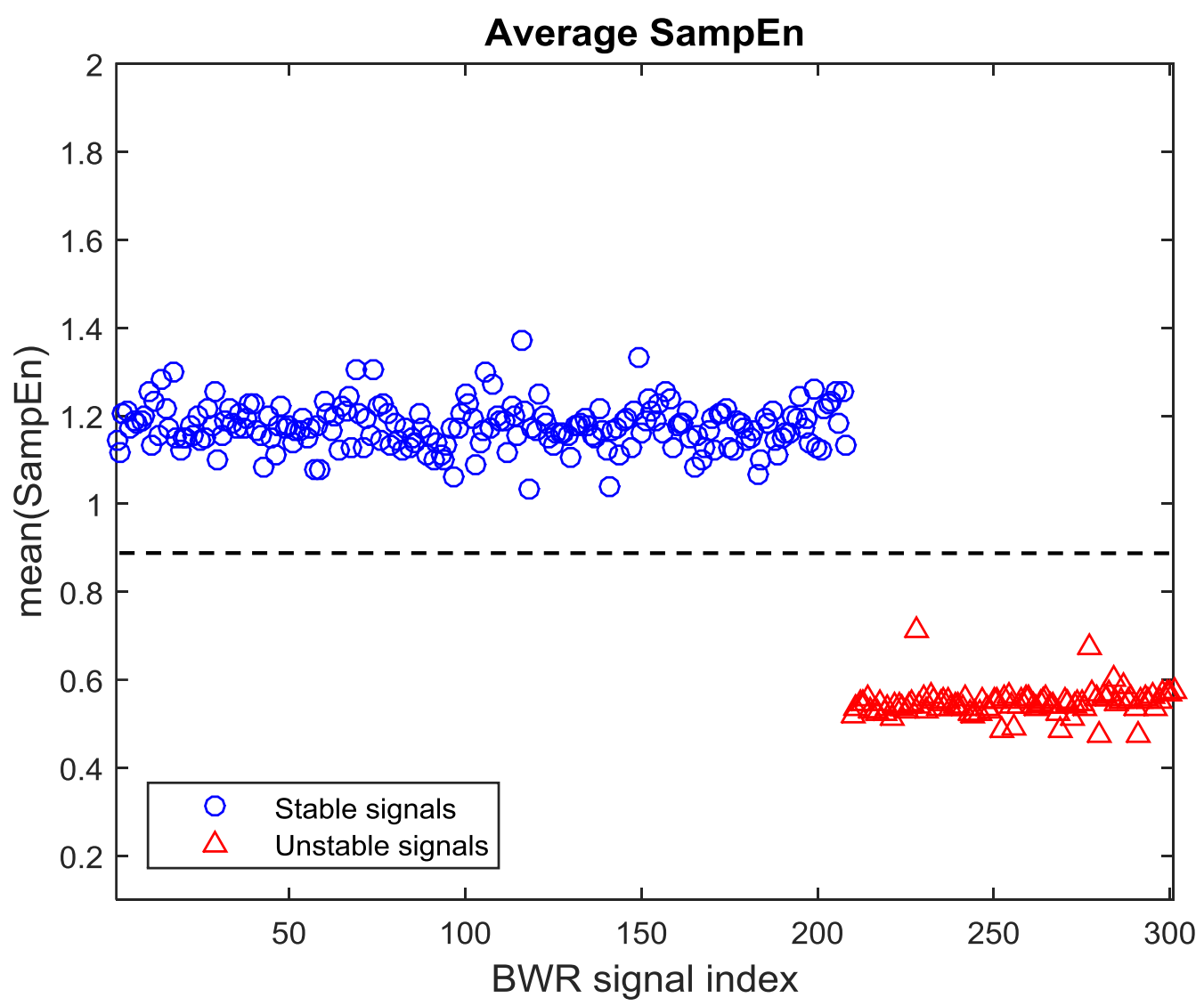

Figure 85. Comparison of the mean(SampEn) values for both studied sets (a stable set of 208 APRM signals versus an unstable set of 96 LPRM signals).

\subsubsection{Case 4 of the Forsmark stability benchmark}

This particular event corresponds to a situtation where the neutronic power reactor suffers unusual unstable problems, i.e., presents a mix of oscillation modes. The C4_APRM and C4_LPRM_x signals correspond to average power monitors (APRM) and local range monitors LPRM. The entire case 4 consists of a total of 23 signals, 22 LPRMs and 1 APRM. Nonetheless, only the analysis of the APRM is presented in this work. Figure 86 shows the studied APRM signal of interest. Figure 87 shows a studied $60 \mathrm{~s}$ seconds segment (the dotted red line) that is decomposed through NA-MEMD and its PSD estimate (the dashed-dotted green line). Figure 88 shows the first 5 extracted IMFs of the studied segment (as a reminder, the IMFs of the 2 noise extra channels are discarded). Figure 89 shows the PSD estimates of the first 6 IMFs, we highlight that there's a bit of mixing between IMF 4 and IMF 5. Nonetheless IMF 5 is slightly closer to $0.5 \mathrm{~Hz}$ than the IMF 4 and has more energy. 
Figure 90 shows a plot of the IMF 5 of interest that is linked to BWR density wave instability and its linked instantaneous frequency (IF) that oscillates around $0.5 \mathrm{~Hz}$ ( we highlight that IMF 5 is slightly mixed with IMF 4 but this is a result due to complexity of the Forsmark case 4. However, IMF 5 has more energy than IMF 4). The SampEn of this IMF 5 is 0.5443 . Thus this segment is unstable (SampEn points to high regularity and low complexity). Figure 91 shows the SampEn estimates of the IMFs or modes of interest along time for all of the studied segments of $60 \mathrm{~s}$. All of the SampEn estimates are smaller than 0.7. So, the studied APRM signal is unstable.

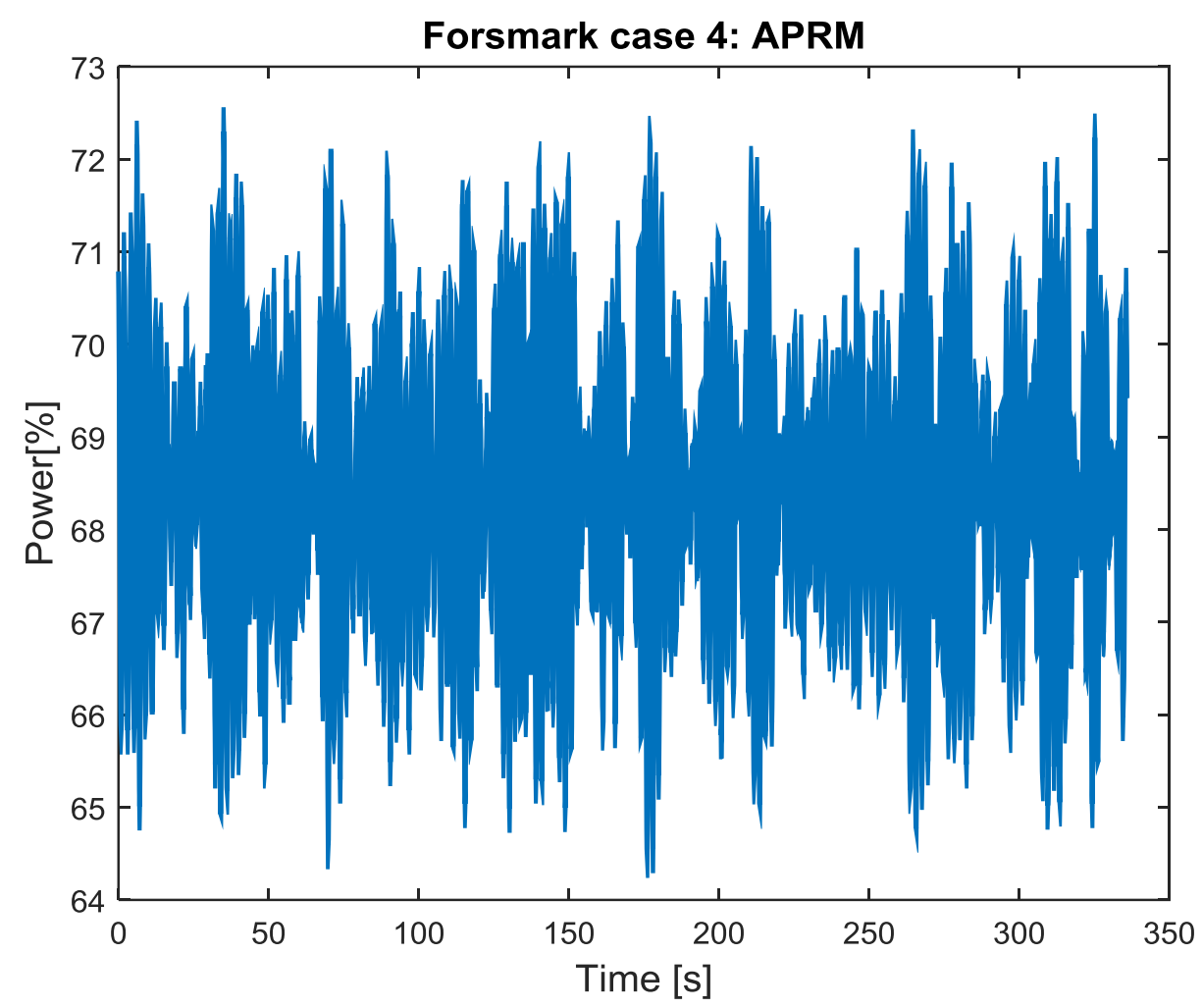

Figure 86. Forsmark Case 4 APRM signal. 

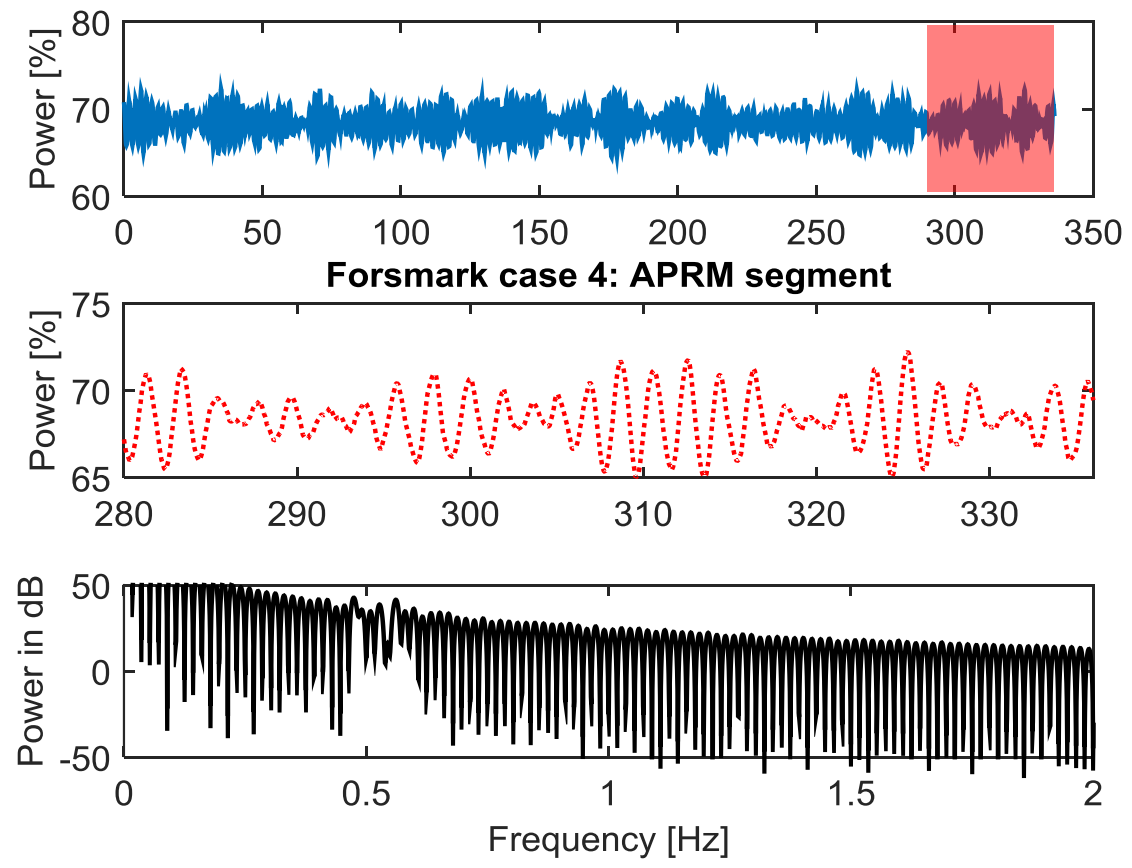

Figure 87. Studied segment of the Forsmark Case 4 APRM.
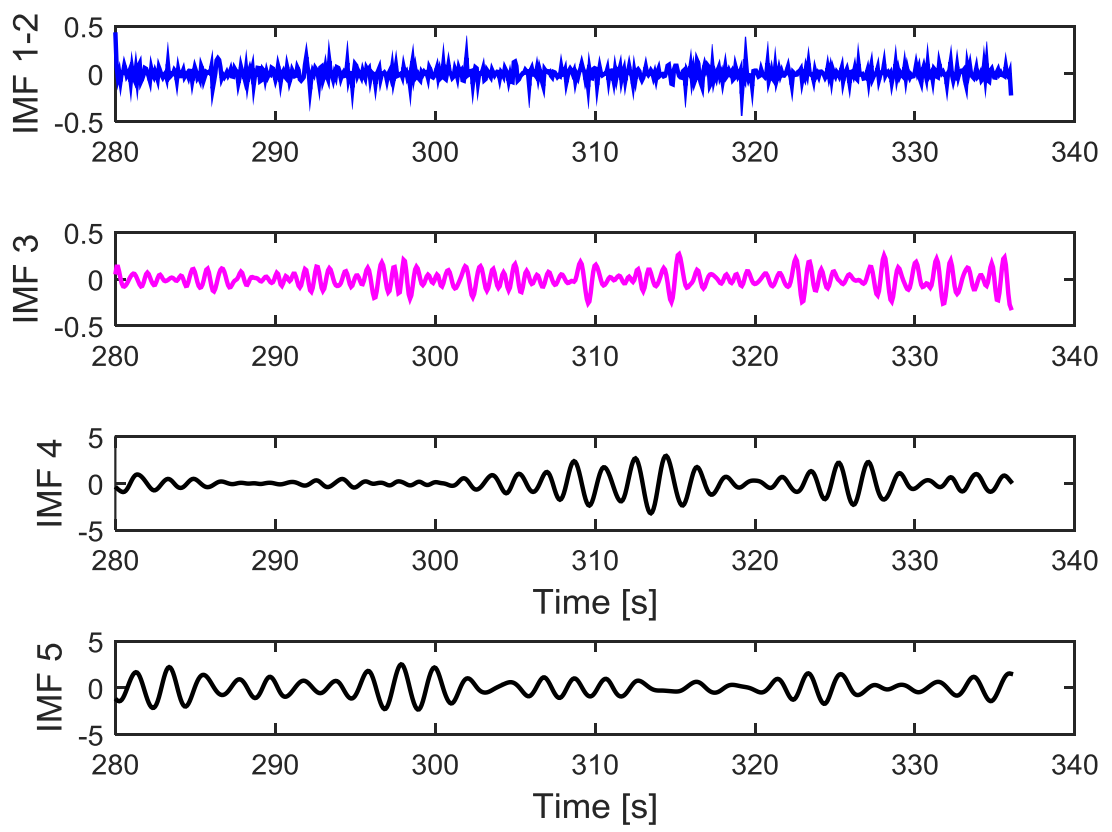

Figure 88. A plot of the first 5 IMFs in a segment of the studied Forsmark APRM. 


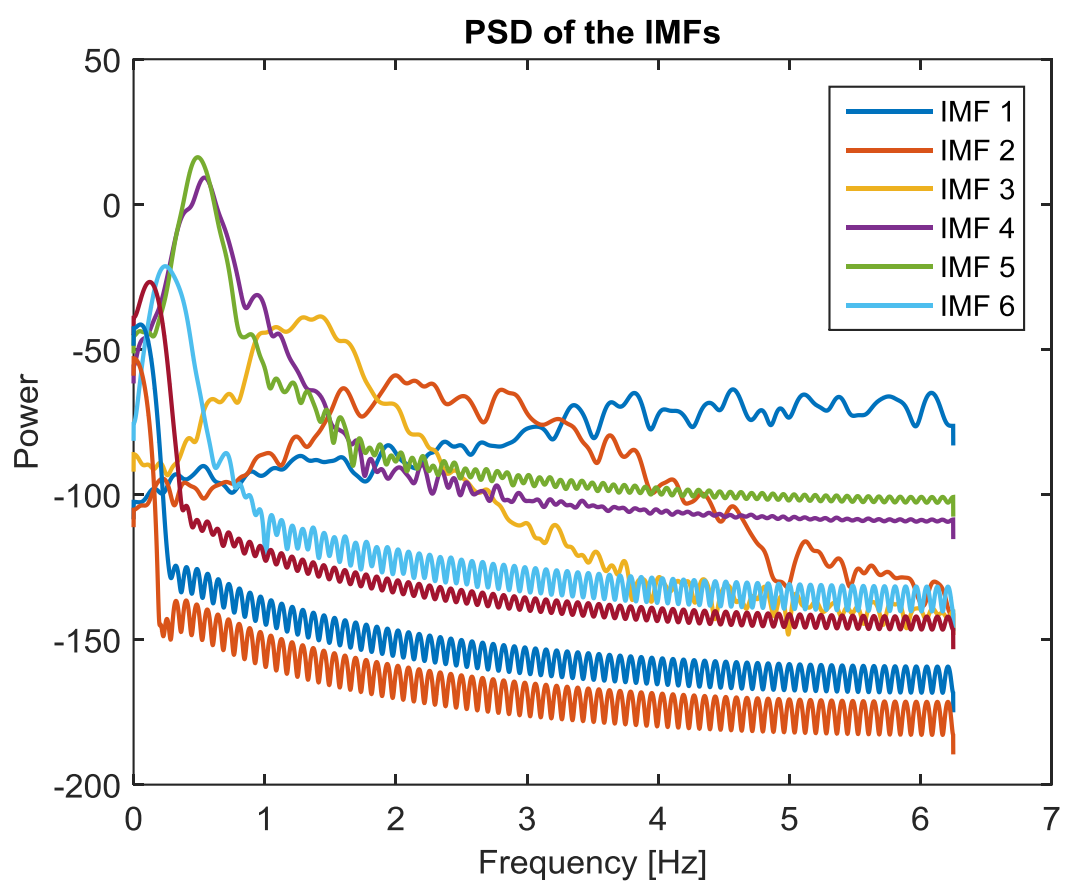

Figure 89. PSD estimate of the first 6 IMFs extracted in the analyzed segment.
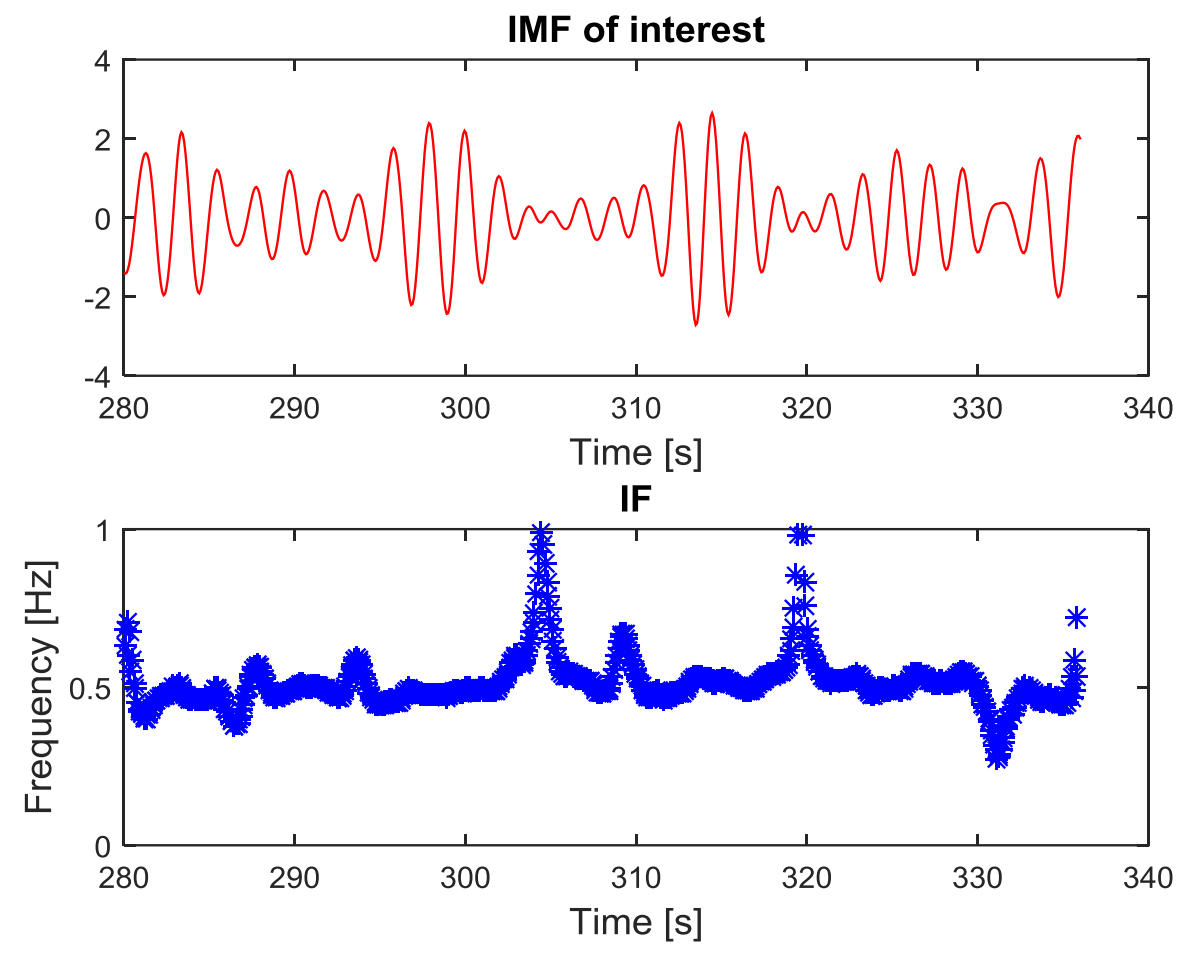

Figure 90. IMF 5 linked to BWR instability and its associated IF (oscillating close to 0.5 $\mathrm{Hz}$ ). 


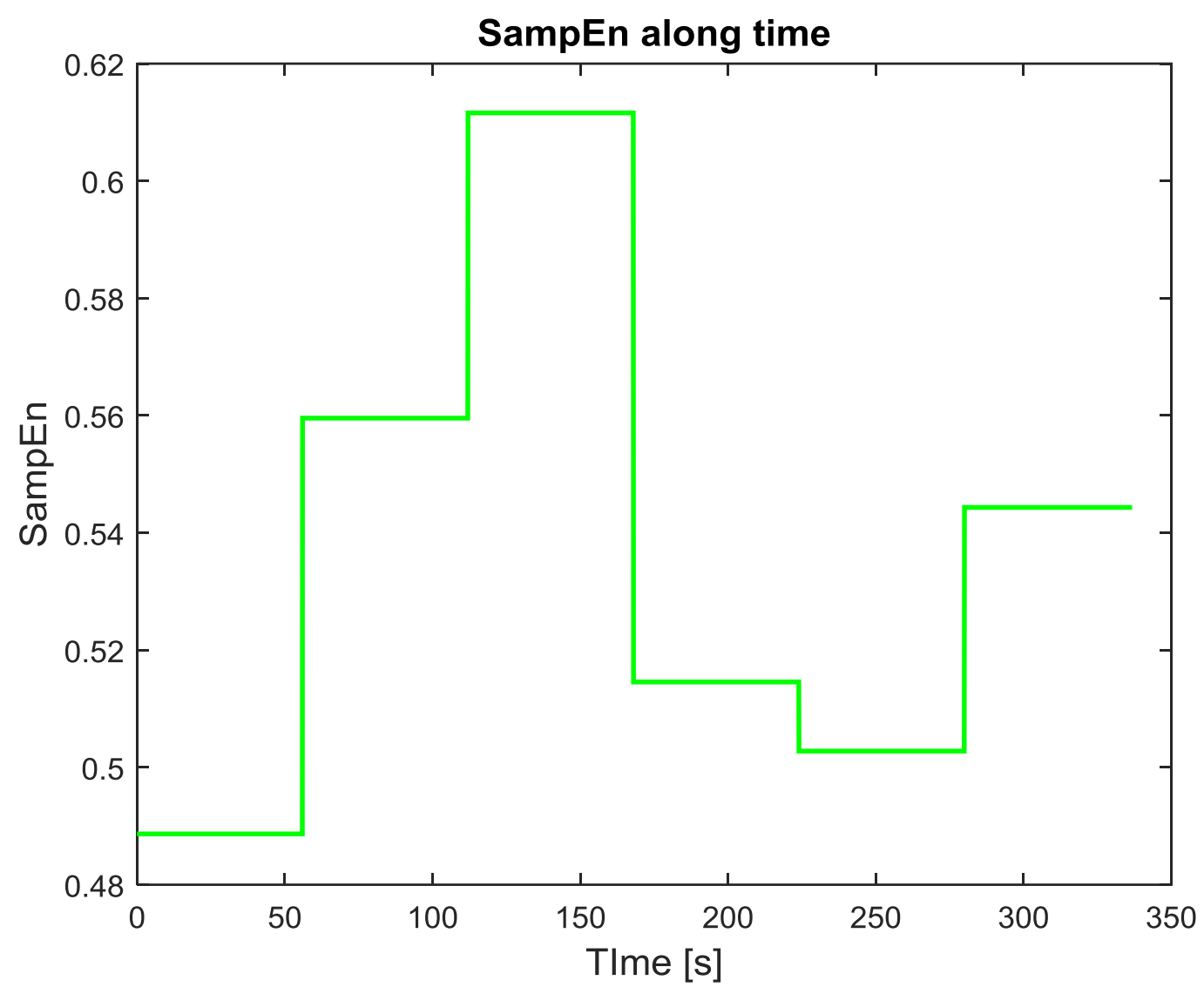

Figure 91. SampEn estimates of the IMFs or modes of interest along time.

Table 13 shows the mean(SampEn), median(SampEn) and std(SampEn) of the time series shown in Figure 91 (SampEn estimates of IMF 4). The mean(IF) and std(IF) are also given in this table. Figure 92 shows a plot of the computed mean SampEn estimates of the 23 unstable signals (each SampEn estimate is a red point). A pattern appears, all of the mean SampEn estimates for the studied signals are smaller than 0.8. Thus, the mean SampEn values point to visually regular signals such as cyclic functions. Through the proposed methodology, we are able to classify these 23 signals in the unstable category with success because their SampEn is smaller than 0.8.

Table 13: Mean(SampEn), Median(SampEn) and std(Sampen) of the analyzed APRM. Now the mean(IF) and std(IF) are included.

\begin{tabular}{lccccc}
\hline Signal & Mean(SampEn) & Median(SampEn) & Std(SampEn) & $\begin{array}{c}\text { Mean(IF) } \\
{[\mathrm{Hz}]}\end{array}$ & std(IF) [Hz] \\
\hline $\begin{array}{l}\text { Forsmark } \\
\text { APRM }\end{array}$ & 0.5369 & 0.5295 & 0.0411 & 0.5017 & 0.0234 \\
\hline
\end{tabular}




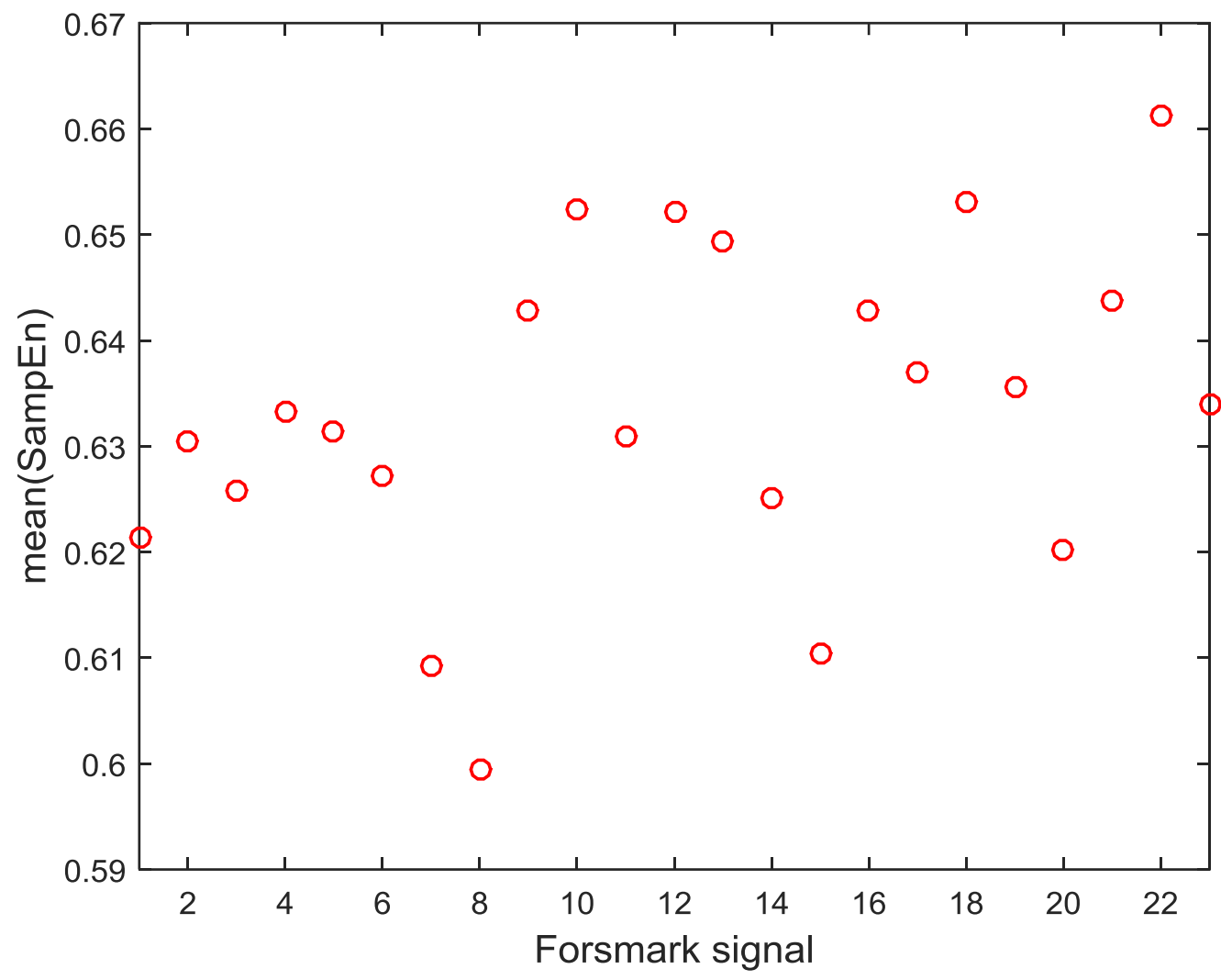

Figure 92. Computed mean(SampEn) of the studied 23 unstable signals (SampEn of the IMFs linked to instability) of the studied Forsmark Case 4.

\subsubsection{Case 9 cycle 14 of the Ringhals stability benchmark}

The data of this case comes from measurements of the Swedish BWR reactor Ringhals 1 . This case consists of a total of 72 LPRMs distributed in two floors or levels of 36 LPRMs each. As stated before, only the analysis of one signal (LPRM 1) is detailed in here. Figure 93 shows a plot of the studied LPRM 1 signal (of level 4). Figure 94 shows a plot of the studied segment (the red dotted line) that is decomposed through NA-MEMD and its PSD estimate (the dashed-dotted green line). Figure 95 shows the first extracted 5 IMFs (by the way, IMF 4 looks like a cyclic function) of the segment. Figure 96 shows a plot of the PSD estimates of the first 6 IMFs. The PSD estimate of IMF 4 is highly concentrated around 0.5 Hz. Figure 97 shows the tracked IMF 4 of interest linked to BWR instability, this IMF 4 looks like a cyclic function and its associated IF is almost a line centered around $0.5 \mathrm{~Hz}$. The computed SampEn of this IMF 4 is 0.4544 (the studied IMF 4 is unstable. Thus, the studied segment is unstable). Figure 98 shows the computations of the SampEn along time for all of the studied segments of $60 \mathrm{~s}$. All of the estimates are smaller than 0.8 and located between 0.4-0.6. So, the studied signal is unstable and highly regular like a cyclic function. 


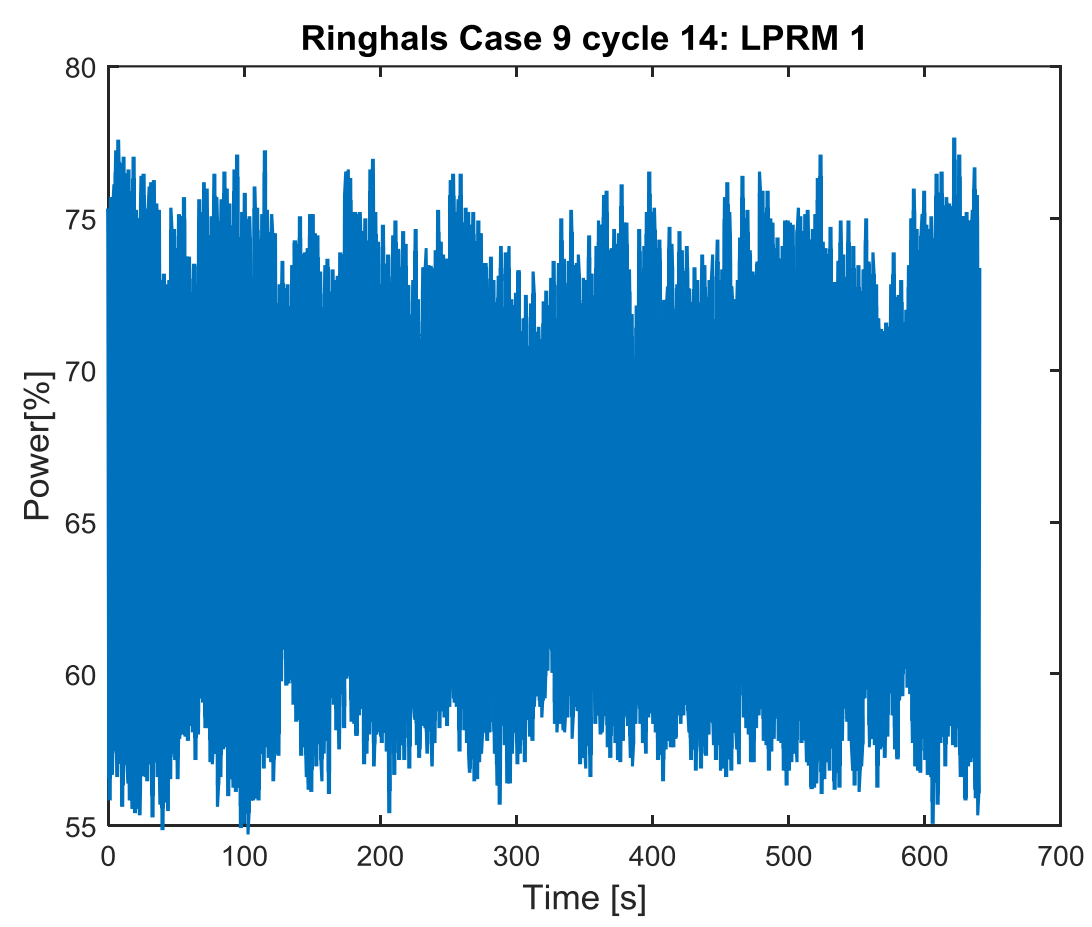

Figure 93. Ringhals Case 9 cycle 14 LPRM1.
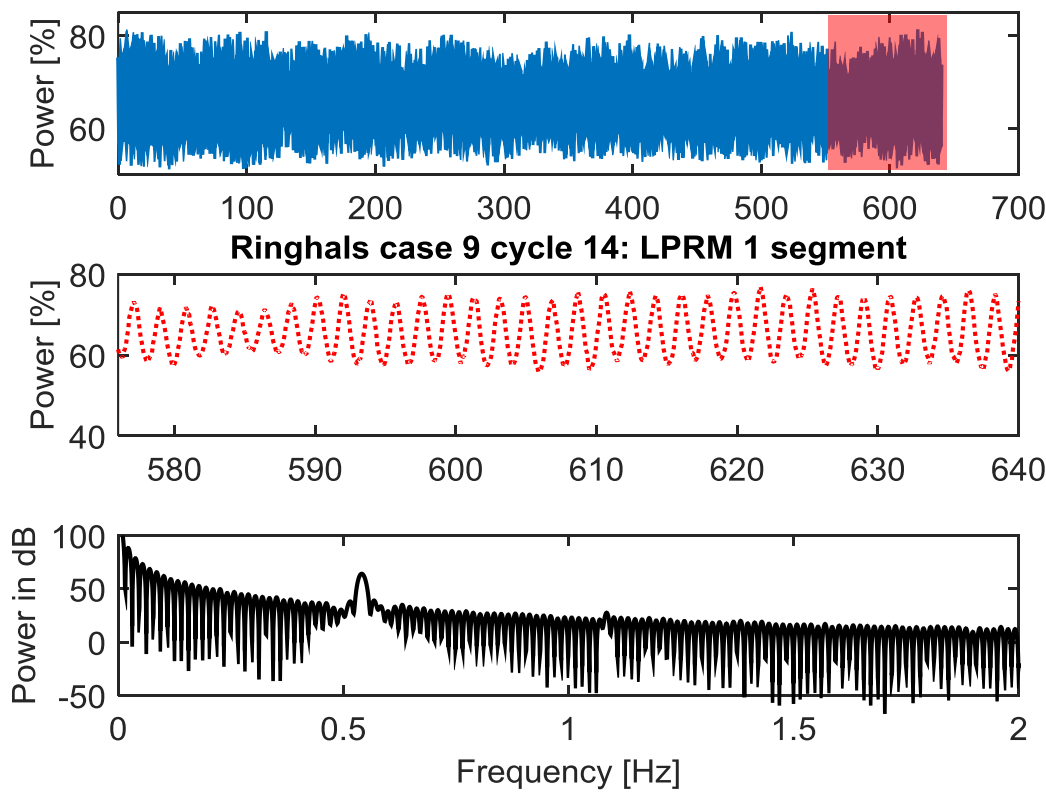

Figure 94. Studied segment of the Ringhals Case 9 cycle 14 LPRM 1. 

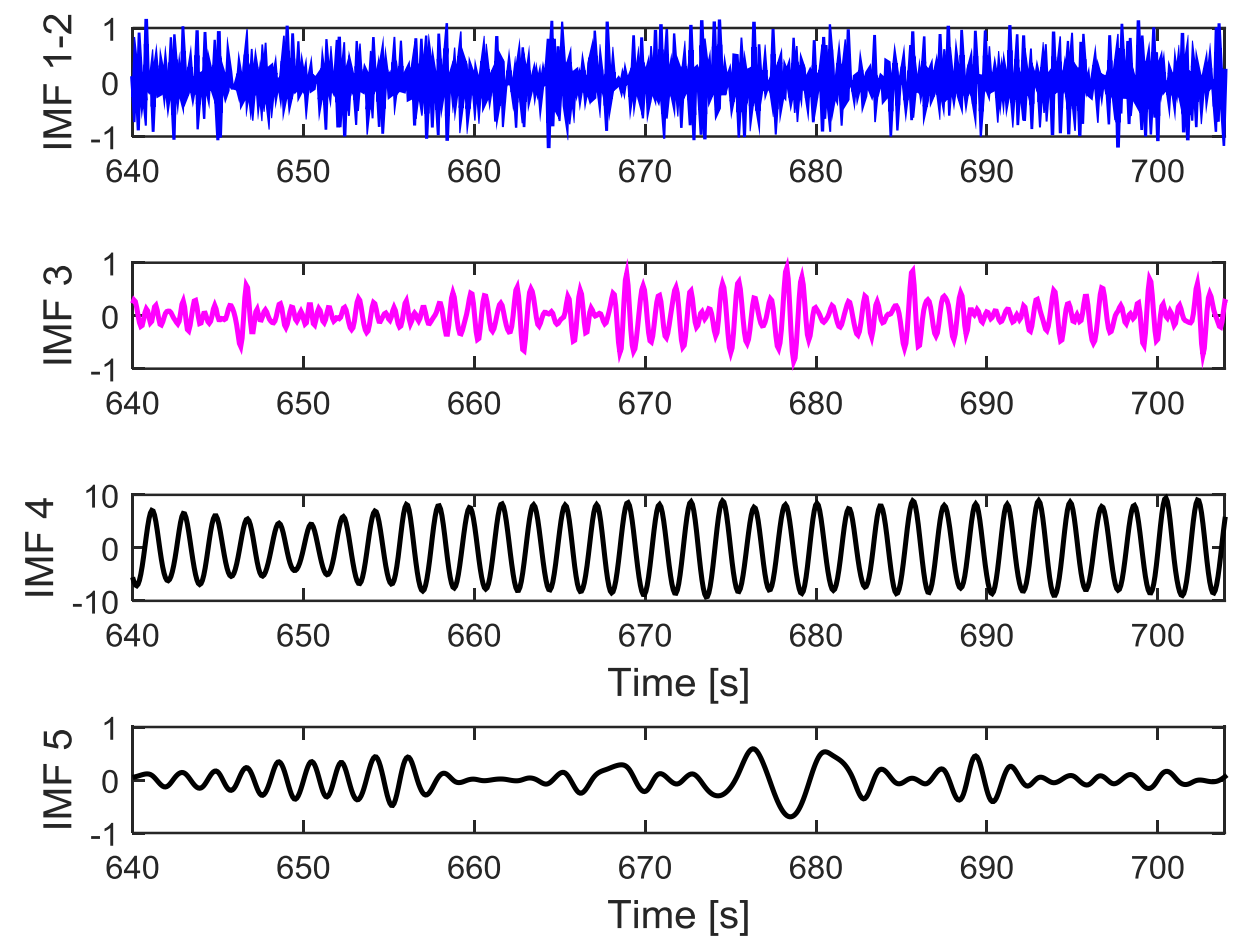

Figure 95. A plot of the first 5 IMFs extracted from the analyzed segment of the studied Ringhals case.

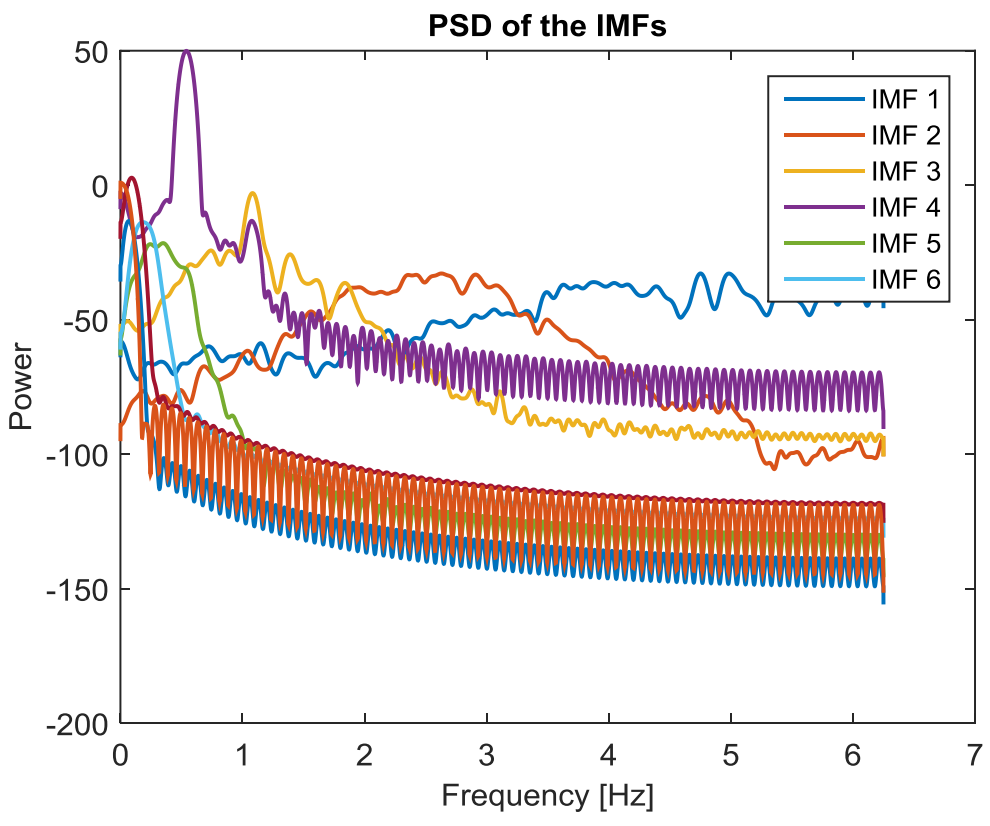

Figure 96. PSD estimate of the first 6 IMFs extracted from the analyzed segment. 

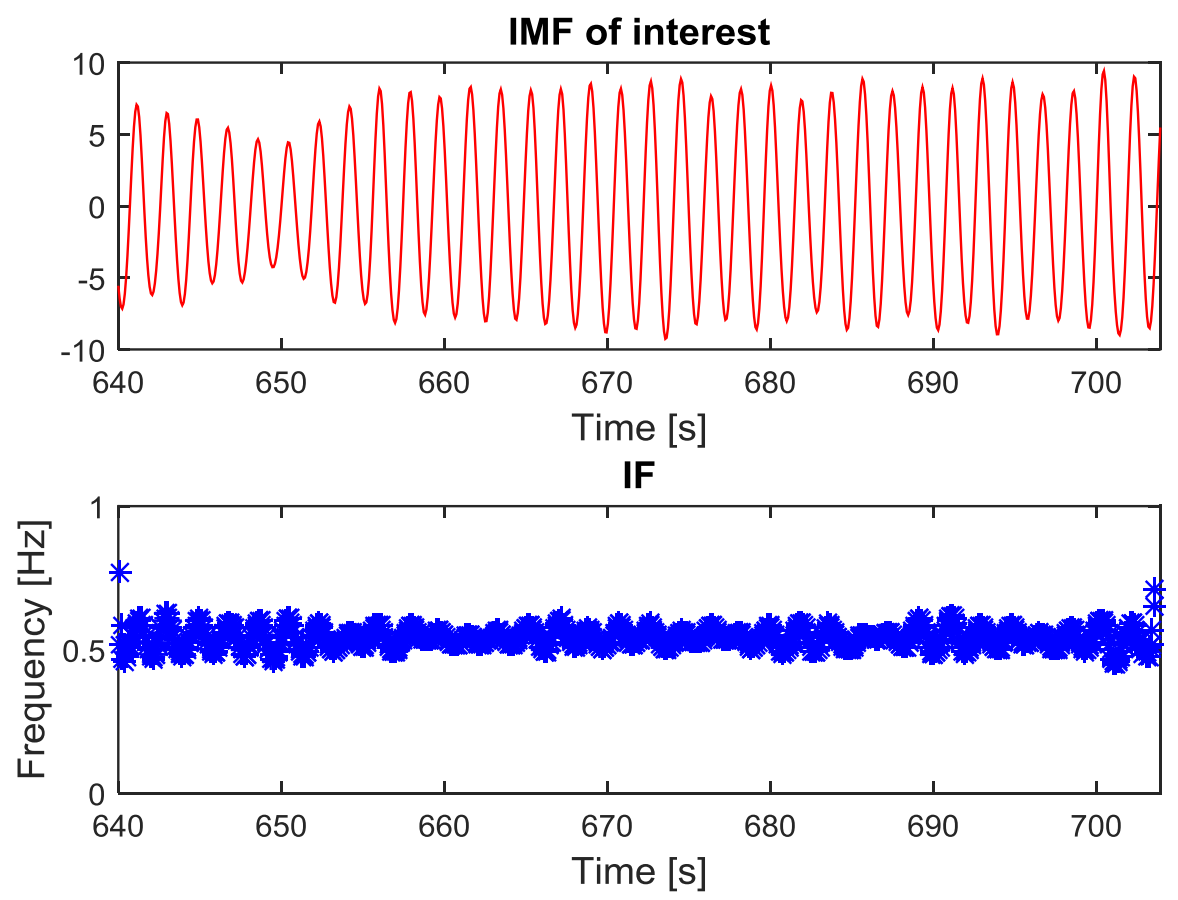

Figure 97. IMF 4 linked to BWR instability and its associated IF (very close to $0.5 \mathrm{~Hz}$ ).

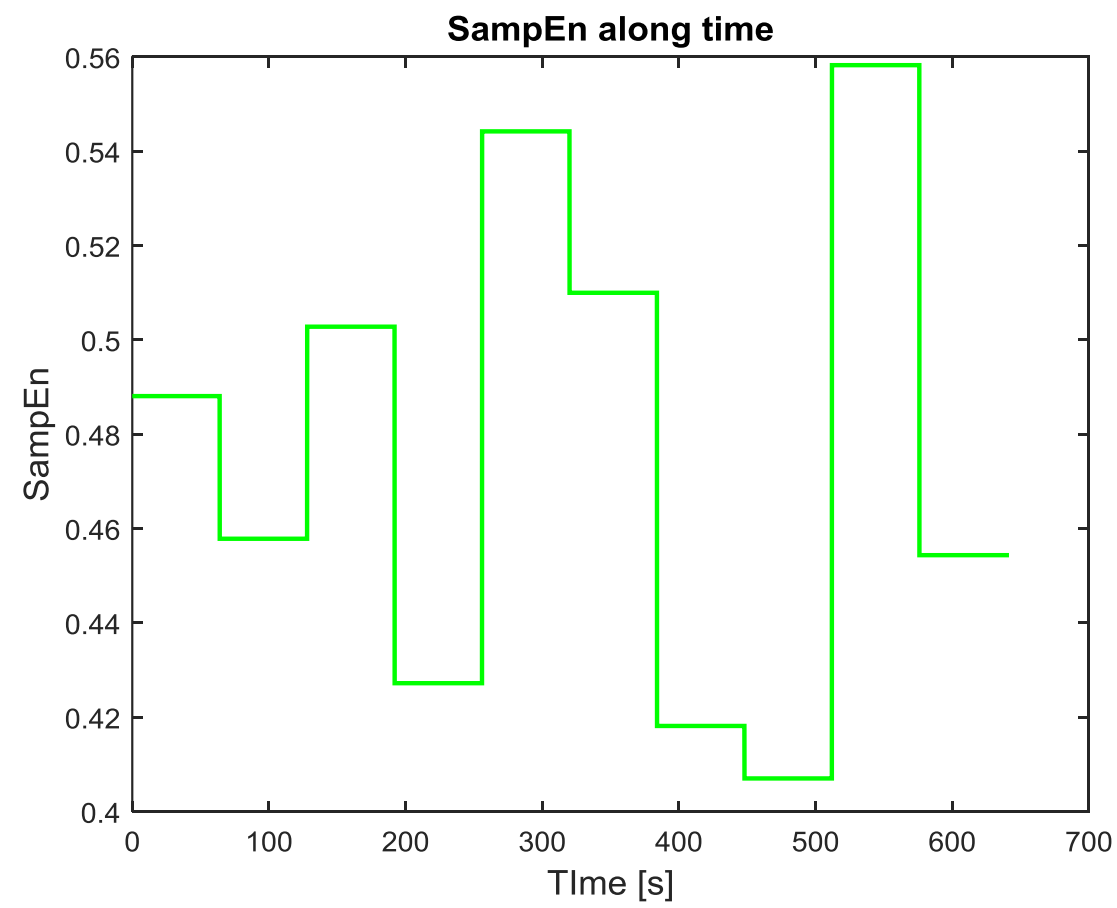

Figure 98. SampEn estimates of the IMFs or modes (IMFs 4) of interest along time. 
Table 14 shows the mean(SampEn), median(SampEn) and std(SampEn) of the time series shown in Figure 98. The mean(IF) and std(IF) of all of the studied segments of the targeted LPRM are also given in this table. Figure 99 shows the computed mean(SampEn) values of the 36 LPRMs of level 2 of the Ringhals reactor. It is observed, that all of the SampEn estimates are smaller than 0.8 (The percentage of classification of the studied signals in the unstable category is of 100\%). The entire floor 2 is unstable and the SampEn estimates are located in the range 0.5-0.7. Figure 100 shows the computed mean(SampEn) values of the 36 LPRMs of level 4 of the Ringhals reactor. It is observed, that all of the SampEn estimates are smaller than 0.8 (The percentage of classification of the studied signals in the unstable category is of $100 \%$ as well). The entire floor 4 is also unstable and the SampEn estimates are located in the range 0.45-0.7. The entire case 9 is unstable and SampEn was perfectly able to detect that behavior.

Table 14: Mean(SampEn), Median(SampEn) and std(Sampen) of the analyzed LPRM 1. Now the mean(IF) and std(IF) are included.

\begin{tabular}{lccccc}
\hline Signal & Mean(SampEn) & Median(SampEn) & Std(SampEn) & $\begin{array}{c}\text { Mean(IF) } \\
{[\mathrm{Hz}]}\end{array}$ & $\begin{array}{c}\text { Std(IF) } \\
{[\mathrm{Hz}]}\end{array}$ \\
\hline Ringhals & 0.4668 & 0.4730 & 0.0497 & 0.5165 & 0.0328 \\
LPRM 1 & & & & & \\
\hline
\end{tabular}

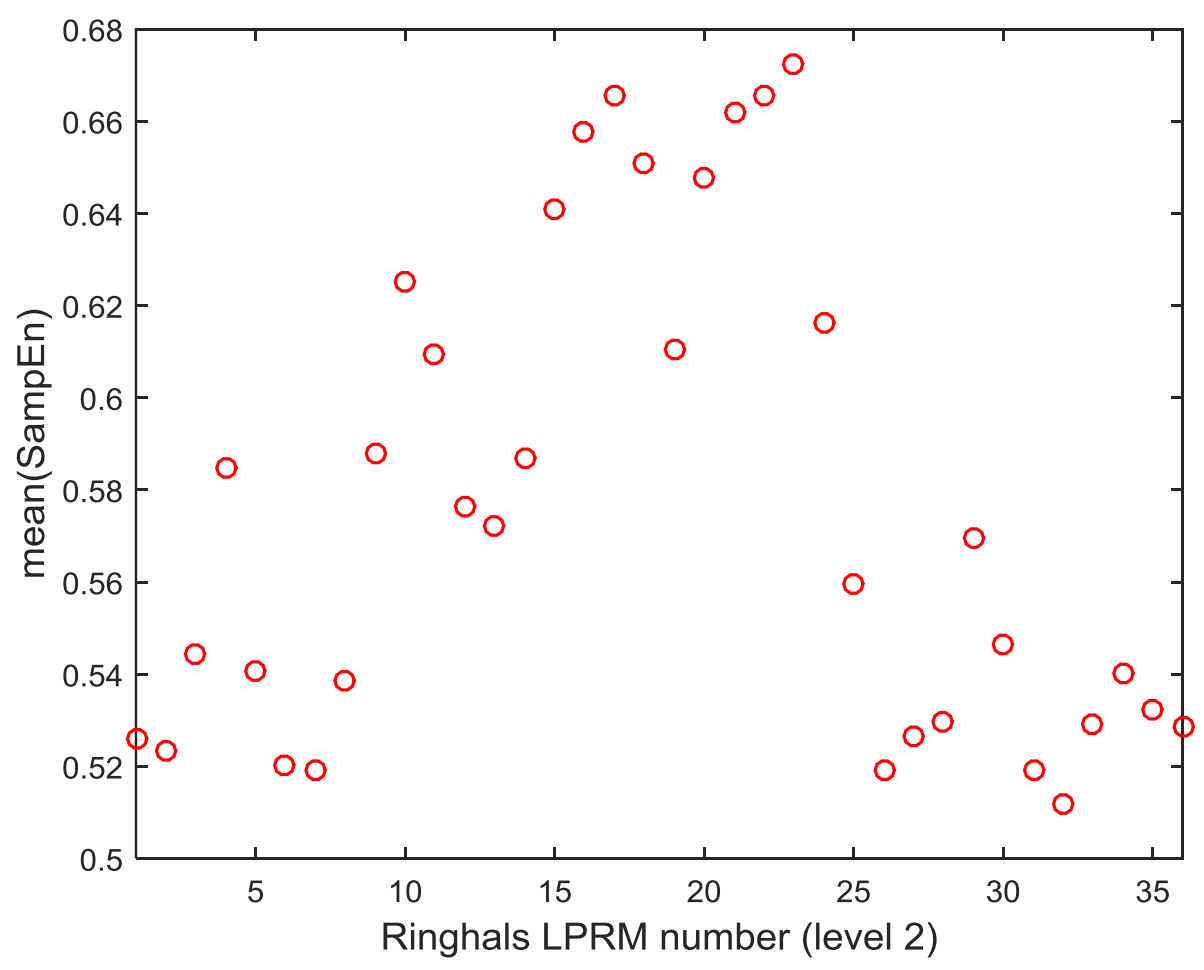

Figure 99. Mean(SampEn) values for the 36 LPRMs of level 2 of the Ringhals BWR unit. 


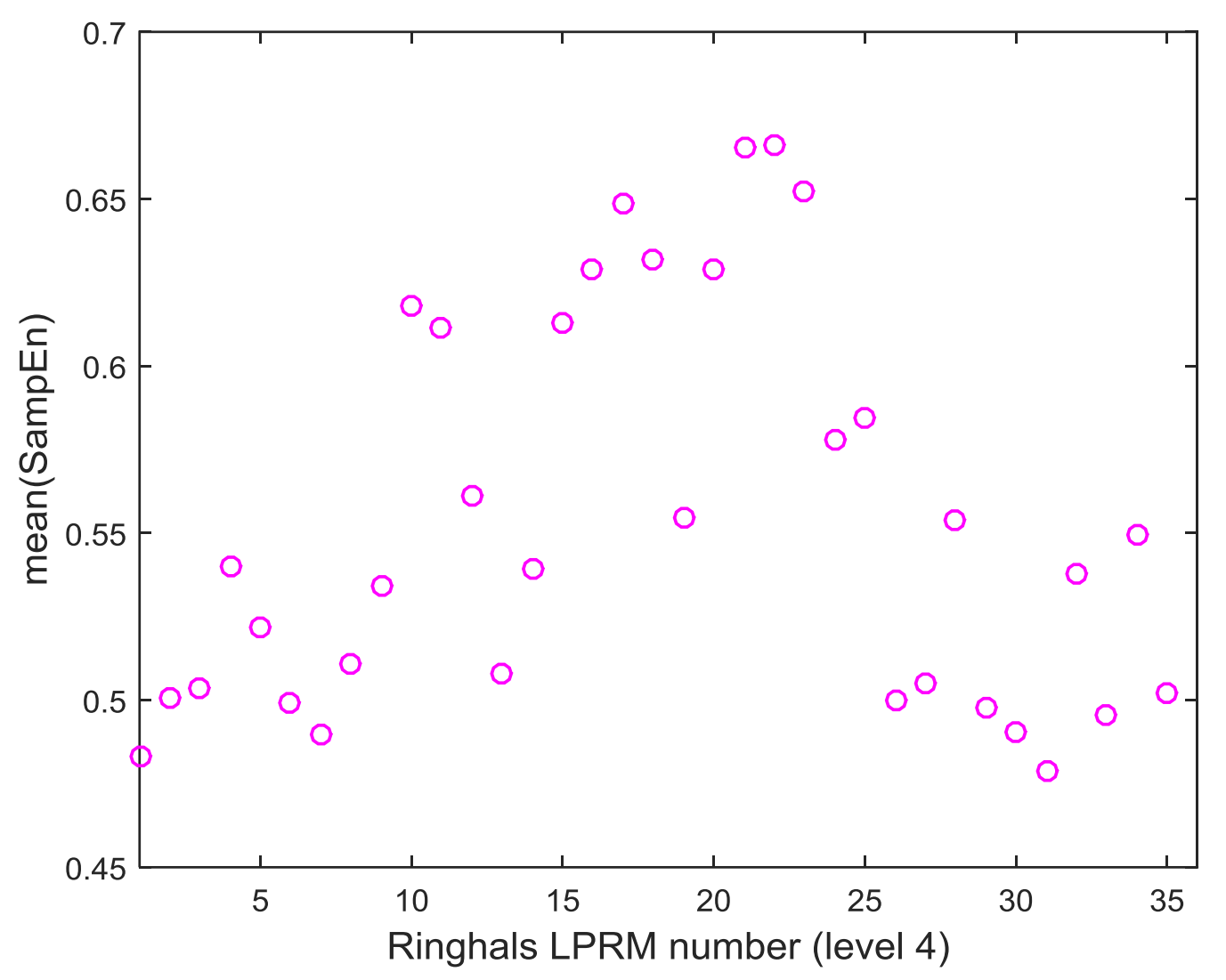

Figure 100. Mean(SampEn) values for the 36 LPRMs of level 4 of the Ringhals BWR unit.

\subsection{Chapter 6 discussions}

In this chapter the Sample Entropy, a measure that provides an index of signal complexity or irregularity of a time series, was used to estimate complexity of a BWR time series for stability analysis. The SampEn was tested in conjunction with the noise-assisted multivariate empirical mode decomposition, a non-linear filter that decomposes nonstationary data from non-linear sources. The NA-MEMD is used in this application to extract correctly the associated mode to the density wave oscillation, before to compute the SampEn. The proposed SampEn + NA-MEMD rolling window methodology allowed categorize stable BWR signals from unstable ones. The computed SampEn values of the stable signals, oscillated around 1.2 (a high SampEN value is linked to a broad band noncoherent noise (BBNCN) associated with stable behavior). The SampEn values of unstable signals fluctuate close to 0.6 (they might be lower than that, but overall such SampEn estimates are below the threshold SampEn value of 0.8). A low value of SampEn points in this case to a simple cyclic function that is linked to density wave instability. $100 \%$ of the typical BWR studied cases were classified with success into stable or unstable categories. $100 \%$ of the Forsmark Case 4 studied LPRMs confirm BWR instability through our proposed SampEn + NA-MEMD methodology. 100\% of the studied LPRMs of level 2 and 
4 of the Ringhals Case 9 cycle 14 confirm instability. According to these experiments, the SampEn paired with the NA-MEMD is a suitable candidate to be used as a BWR stability indicator. In the future, the SampEn might be tested in conjunction with other suitable nonlinear indicators (possibly fractal dimension estimators, as in Demazière et al., 2008) to build a robust BWR stability monitor based on 2 non-linear measurements, where one of them is going to be the SampEn. In general, the SampEn is a suitable and easy to implement BWR non-linear stability indicator for real time series analysis provided that there is enough data for SampEn computation. The SampEn requires sufficiently large data points to provide reliable estimates, by far the time series length constraint is the only handicap of the SampEn technique. 


\section{Chapter 7}

\section{BWR stability indicator 3: The Higuchi fractal dimension}

In this chapter, the Higuchi fractal dimension (HFD, Higuchi, 1988) is explored to assess BWR instability due to density waves. The HFD is a non-linear measure of signal complexity in the time domain. The HFD is a numerical approach to compute the fractal dimension (FD), a tool that stems from fractal theory to provide an index of roughness of a pattern (i.e., a fractal pattern) by comparing how a detail in a particular pattern changes with the scale at which it is measured. The HFD is a fraction on the interval $1<$ HFD $<2$, where a low value of HFD indicates a predictable and regular time series whereas a high HFD indicates an unpredictable and very irregular time series (such as noise). In the BWR stability context, the HFD computations are aimed towards the detection and classification of stable states from unstable ones. The HFD based stability methodology for BWR developed in this chapter was tested with the real signals studied in past chapters.

The HFD has been applied successfully in different areas of neurophysiology, from cellular to systems neurophysiology. The HFD was used for single neuron ( e.g., Spasić et al., 2011; Kesić et al., 2014), in EEG (e.g., Spasić et al., 2005a,b), and in magnetoencephalographic (MEG) signal analysis (e.g., Gómez et al., 2009; Gómez and Hornero, 2010; Poza et al., 2012)

However, further application of HFD in basic research and medical practice will reveal its complementarity with, advantages or disadvantages over existing linear and non-linear methods (Kesić and Spasić, 2016). When it comes to the test of non-linear fractal (Mandelbrot, 1983) based methods on the domain of BWR stability, various techniques have been tested before, such as the correlation exponent (Grassberger and Procaccia, 2004) which is a characteristic measure of strange attractors and allows to differentiate between different strange attractors (Strogatz, 1994) which allows one to distinguish between deterministic chaos and random noise. The usefulness of the correlation exponent in characterizing experimental data which stems from very high dimensional systems is highlighted in the BWR stability domain in (e.g., Suzudo 2003; Suzudo et al., 1993; Otero et al., 2003) through a practical technique to compute the correlation exponent (Grassberger and Procaccia, 2004). 
Nonetheless, this practical method requires a set of parameters that are not known a priori related to the studied data, such as embedding dimension, time delay, Theilers corrector (Theiler, 1990), the number of singular values (SV) and the generalization index. Even if optimal methods to compute the five aforementioned parameters are implemented in BWR stability proposals, their inclusion will increase the cost of the methodology proposed. Based on these facts, we explore more practical estimation methods based on fractal theory, and with less restriction involved in the computation of the input parameters of the studied techniques, to develop new stability methodologies where a fractal measurement is involved to provide information related to the status of reactor signals.

\subsection{What are fractals?}

Fractals are complex geometric objects with a particularly property (in general): selfsimilarity, i.e. a pattern is repeated in an embedded way at different scales. Sometimes such similarity is exact; more often it is only an approximate or statistical. Many natural structures are fractal-like such as mountains, clouds, coastlines, blood vessel networks, etc. In Figure 101 are displayed two classic fractals: one obtained from the Maldelbrot set (Mandelbrot, 1983) and the Von Koch curve (both generated from Matlab). The fractals obtained from the Mandelbrot set are beautiful images (even there were considered as Art) and the Von Koch curve looks like a rough but vigorous model of a coastline, wrote Mandelbrot to describe it. Further details related to the scientific applications of fractals, and their lovely mathematical theory behind them can be found in (Mandelbrot, 1983).

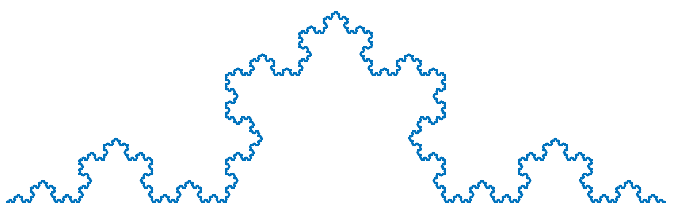

Von Koch curve

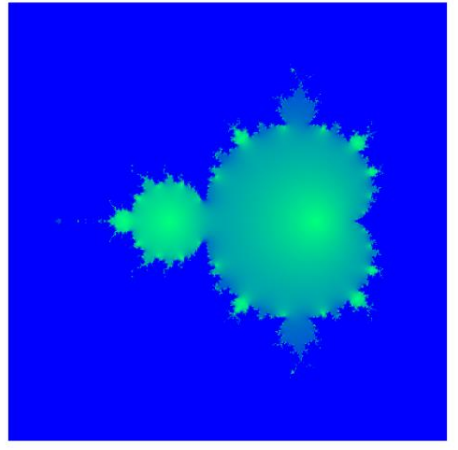

Mandelbrot set

Figure 101. Examples of fractals.

The mathematical key property of a genuinely fractal object is that its fractal metric dimension is a non-integer rational number called the fractal dimension (FD), a measure that can be used to characterize the shape of this object. In the topological sense, a curve in a plane always has a dimension of one. On the other hand, as the curve becomes more and more complex, the curve fills more and more the plane. Thus, in another sense, a complex curve can be considered to have a FD of greater than one. In terms of fractal dimension, a 
straight line has a dimension of one, an irregular line has a dimension between one and two, and a complex curve completely filling this plane could have a dimension approaching of two. Besides, lines accumulating repeatedly in the plane by crossing and recrossing themselves, can even have fractal dimension of greater than two (Katz and George, 1985). In this way, fractal dimensions can be used to assign numbers to the degree of complexity of planar curves. For example, the von Koch curve Fractal Dimension (FD) is approximately 1.26 (Strogatz, 1994). This measure is greater than the dimension of a line (1.0) and greater that the dimension of the Cantor set (0.63) (Fleron, 1994), which means that the Koch curve is rougher (i.e. more complex and intricate) than the Cantor set. Then, the FD is a natural measure of irregularity of a curve, in our case, a BWR time series that can be either stable or unstable depending on its irregularity.

\subsection{The Higuchi fractal dimension algorithm}

The FD estimator that is used in this work is the Higuchi Fractal Dimension (HFD), a nonlinear tool permitting to measure the complexity of a curve, in special to measure regularity of discrete-time series. This method was proposed by Higuchi, 1998 and it is summarized in the next steps:

Step1. Given $x(n)$, a $N$-points discrete-time series $x(1), x(2), \ldots, x(N)$; construct a new selfsimilar time series $x_{k}(m)$ as:

$$
x_{k}(m) \rightarrow\left\{x(m), x(m+k), x(m+2 k), \ldots, x\left(m+\operatorname{int}\left[\frac{(N-k)}{k}\right] k\right)\right\}
$$

with $m=1,2, \ldots, k$ being the initial time; $k$ is the time interval, $k=1, \ldots, \mathrm{k}_{\max }$ is a free parameter, and int $[r]$ is the integer part of the real number $r$.

Step 2. For each of the $k$ time series (or curves) $x_{k}(m)$, compute the length $L_{m}(k)$ as:

$$
L_{m}(k)=\frac{1}{k}\left[\left(\sum_{i=1}^{\operatorname{int}\left[\frac{N-m}{k}\right]}|x(m+i k)-x(m+(i-1) k)|\right) \frac{N-1}{\operatorname{int}\left[\frac{N-m}{k}\right] k}\right]
$$

where $(N-1) /(\operatorname{int}[(N-m) / k] k)$ is a normalization factor. 
Step 3. Obtain an array of mean values $L(k)$ for each $k=1, \ldots, \mathrm{k}_{\max }$ averaging $L_{m}(k)$ for all $m$ as:

$$
L(k)=\frac{\sum_{m=1}^{k} L_{m}(k)}{k}
$$

Step 4. Finally, estimate the HFD from the slope of the best least squares linear fitting the plot $\ln (L(k))$ versus $\ln (1 / k)$, i.e.

$$
\mathrm{HFD}=\frac{\ln (L(k))}{\ln (1 / k)}
$$

In the practical cases, the original time series can be segmented into smaller windows, overlapped or not. In this case, the method for computing HFD values should be applied to each window individually, with $N$ representing the length of each window. The mean of all HFD values for these windows can be used as a measure of complexity for the entire signal.

In the case of the BWR signals, the HFD for a random white noise (stable state) will be estimated around $\approx 1.5$ or higher, and for a low frequency sine wave around $0.5 \mathrm{~Hz}$ (instable state) will be $\approx 1$ (HFD estimated value corresponding to a smooth curve).

\subsection{How to select an adequate kmax free parameter value?}

The HFD has only one free parameter noted as $\mathrm{k}_{\max }$ in the method previously described being his choice very important in the algorithm performance. Higuchi, 1988 did not elaborate extensively in his work on the selection of $\mathrm{k}_{\max }$ value, however some efforts have been developed in order to get the most suitable $\mathrm{k}_{\max }$ in according with the application (e.g. Accardo et al., 1997; Virkkala et al., 2002; Olejarczyk, 2007; Gómez and Hornero, 2010).

In this work, various numerical experiments were performed with synthetic signals with well-known theoretical values of FD in order to figure out a quasi-optimal fixed $\mathrm{k}_{\max }$ value. Such test experiments not only provide information related to the accuracy of the HFD, but also give information linked to the choosing of a quasi-optimal $\mathrm{k}_{\max }$ value, which is used for HFD estimates of BWR data. These experiments are next presented. 


\subsubsection{Synthetic signals: Fractional Gaussian noise}

In this subsection, the reliability of the HFD is tested with a set of complex noise signal paths with well defined values of fractal dimension. Our goal in mind is to infer an adequate estimate of $\mathrm{k}_{\max }$ to calibrate the HFD to suit the intended goal of proposing a new BWR stability indicator able to provide the most accurate FD estimates HFD can provide for our real world application.

Such complex signals are variations of fractional Gaussian noise (fGn) paths, which are defined as the increment process of fractional Brownian motion (Dieker, 2004). In discretetime, any fGn path corresponds to a time series $\left\{x_{\mathrm{H}}(n), n=\cdots,-1,0,1, \cdots\right\}$ indexed by a real-valued parameter $0<\mathrm{H}<1$, and its autocorrelation function is given by:

$$
\begin{aligned}
r_{x_{\mathrm{H}}}(k) & =E\left[x_{\mathrm{H}}(n) x_{\mathrm{H}}(n-k)\right] \\
& =\frac{\sigma^{2}}{2}\left(|k-1|^{2 \mathrm{H}}-2|k|^{2 \mathrm{H}}+|k+1|^{2 \mathrm{H}}\right)
\end{aligned}
$$

where $\mathrm{H}$ is the Hurst exponent (Hurst, 1951) and $\sigma^{2}$ is the variance of the noise. The Hurst exponent is a dimensionless estimator for the self-similarity of a time series. The Hurst exponent is related to the fractal dimension (FD) by the next simple relation:

$$
\mathrm{H}=2-\mathrm{FD}
$$

In fact, FD and $\mathrm{H}$ are cousins measuring irregularity of a curve (in our context, a time series). For a very irregular time series $\mathrm{H}=0$, for white noise $\mathrm{H} \approx 0.5$ (Wood and Chan, 1994) and $\mathrm{H}=1$ indicates a predictable direct motion, coming from a regular time series, possibly a smooth curve. In previous works further information about $\mathrm{H}$ is presented (e.g., Rasheed and Qian, 2004; Mandelbrot and Hudson, 2010). The special case $H=0.5$ reduces to white noise, whereas other values induce non-zero correlations, either negative when $0<\mathrm{H}<0.5$ or positive when $0.5<\mathrm{H}<1$ that represent long-range dependence. In here, intensive simulations have been performed on such processes, with $\mathrm{H}$ varying from 0.1 to 0.9 to build a set $s_{i} \quad i=1,2, \ldots, 100$ of 100 signals of fGn. The data length of each signal in the set is $N=512$. The paths of fGn have been generated by the method given by Wood and Chan, 1994. Figure 102 shows for illustrative purposes the plot of $8 \mathrm{fGn}$ signal paths where $\mathrm{H}$ starts to increase from 0.1 (Figure 102A) until reaching the value 0.8 (Figure $102 \mathrm{H})$. With each increment of $\mathrm{H}$ the fGn paths become more regular (i.e. less rough and less complex) and predictable. The FD decreases accordingly from 1.9 to 1.2. 

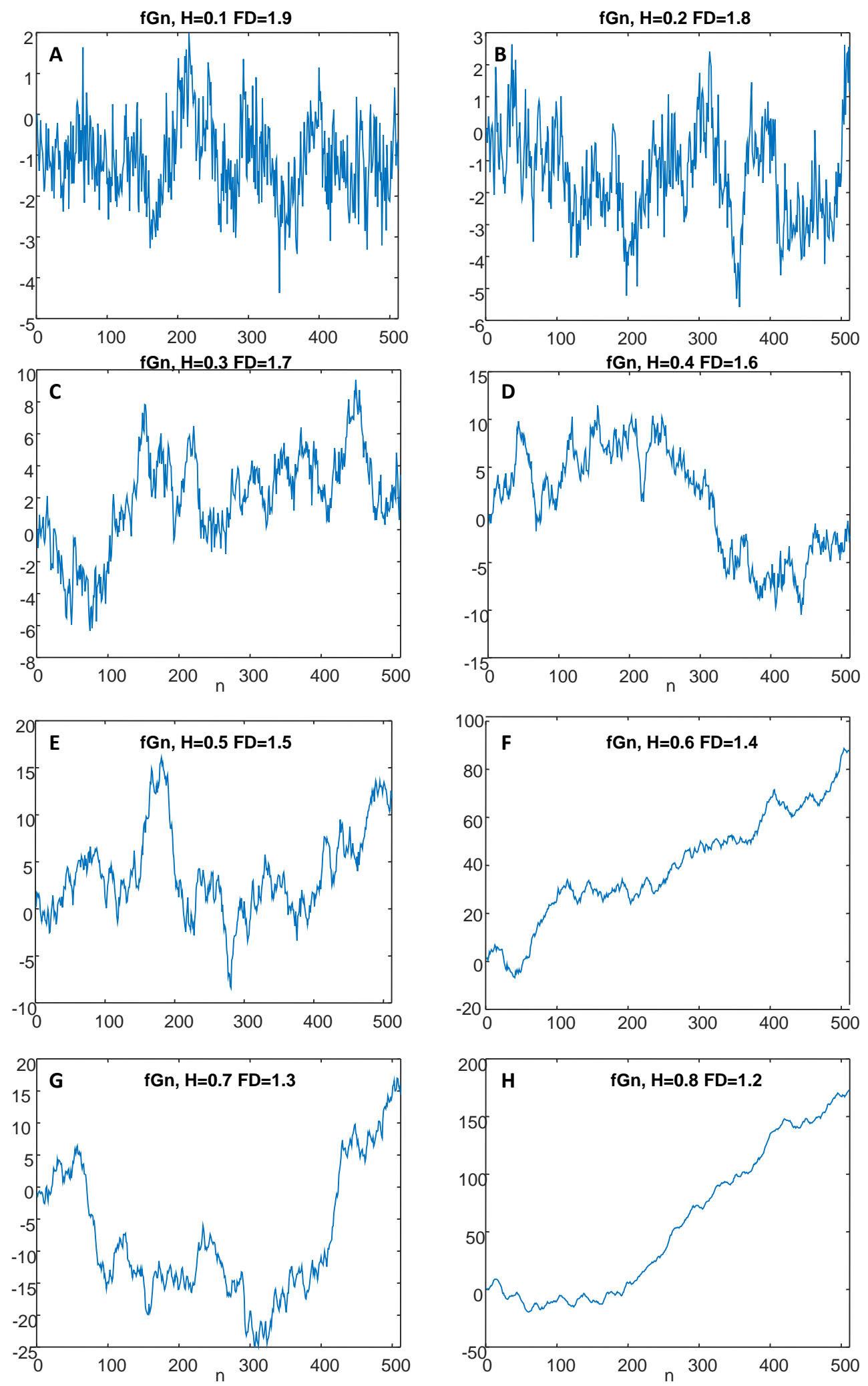

Figure 102. fGn signals generated for 8 specific Hurst exponent ( $H$ ) values. 
For the next experiment, that now involves the FD estimation through the practical HFD technique, a set of $100 \mathrm{fGn}$ signal paths $s_{i}(i=1, \ldots, 100)$ of length $N=512$, were generated. $\mathrm{H}$ varies in the interval $\mathrm{H}=0.1+\Delta H, 0.1+2 \Delta H, \ldots, 0.9$ with $\Delta \mathrm{H}=1 / 100$. For each fGn signal in the set, the HFD is computed, for integer values of the free parameter $\mathrm{k}_{\max }$ chosen in the interval $[2,3,4, \ldots, 32]$ (for $\mathrm{k}_{\max }=1$ the HFD is nonexistent, so this value is ignored for our simulations).

This step will give us the next matrix $G_{\mathrm{H} \times \mathrm{k}_{\max }}$ :

$$
\mathrm{H}\{G_{\mathrm{H} \times \mathrm{k}_{\text {max }}}=\overbrace{\left(\begin{array}{cccc}
\operatorname{HFD}\left(s_{1}, 2\right) & \operatorname{HFD}\left(s_{1}, 3\right) & \cdots & \operatorname{HFD}\left(s_{1}, 32\right) \\
\operatorname{HFD}\left(s_{2}, 2\right) & \operatorname{HFD}\left(s_{2}, 3\right) & \cdots & \operatorname{HFD}\left(s_{2}, 32\right) \\
\vdots & \vdots & \vdots & \vdots \\
\operatorname{kFD}\left(s_{100}, 2\right) & \operatorname{HFD}\left(s_{100}, 3\right) & \cdots & \operatorname{HFD}\left(s_{100}, 32\right)
\end{array}\right)}^{\mathrm{k}_{\text {max }}}
$$

Each column of the matrix $G_{\mathrm{H} \times \mathrm{k}_{\max }}$ gives us a line of computed values of the HFD, as shown in Figure 103, the blue straight line is the theoretical FD line of the fGn paths in the set (this is the line this experiment is trying to approach through HFD for a particular fixed value of $\mathrm{k}_{\max }$ ). In this figure, only 5 HFD lines (instead of the 32 different lines) are plotted for illustrative purposes.

The mean square error (MSE) between the theoretical line of FD values and each column of $G_{\mathrm{H} \times \mathrm{k}_{\max }}$ is computed. Each $G_{\mathrm{H} \times \mathrm{k}_{\max }}$ column generates a line of HFD estimates for a fixed $\mathrm{k}_{\max }$ value. This MSE between the desired blue line and each generated line of HFD estimates is shown in Figure 104. The best $\mathrm{k}_{\max }$ is the one that gives us the line that is the closest to the theoretical FD blue line. This line is also linked to the smallest MSE in the plot. The line that best approaches the theoretical blue line is the one where the HFD was computed for $\mathrm{k}_{\max }=4$ as is depicted in Figure 105. 


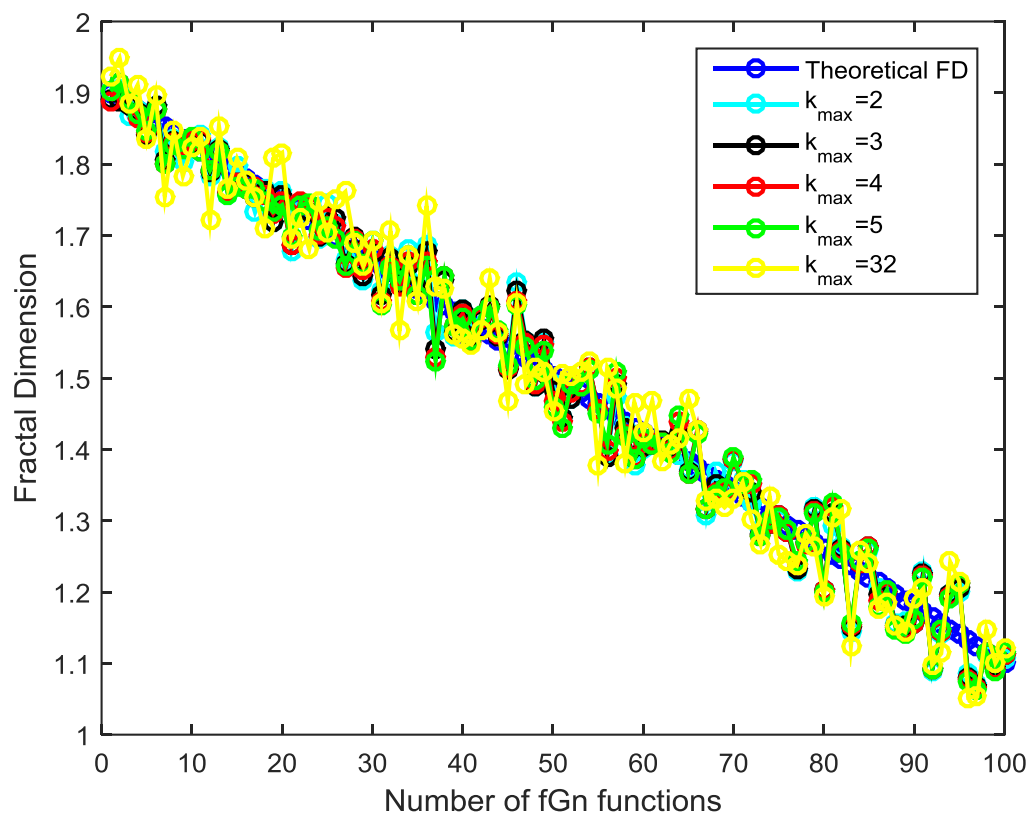

Figure 103. Plot of five columns of $G_{\mathrm{H} \times \mathrm{k}_{\max }}$. Each column grants a pseudo-straight line for a particular $\mathrm{k}_{\max }$ value.

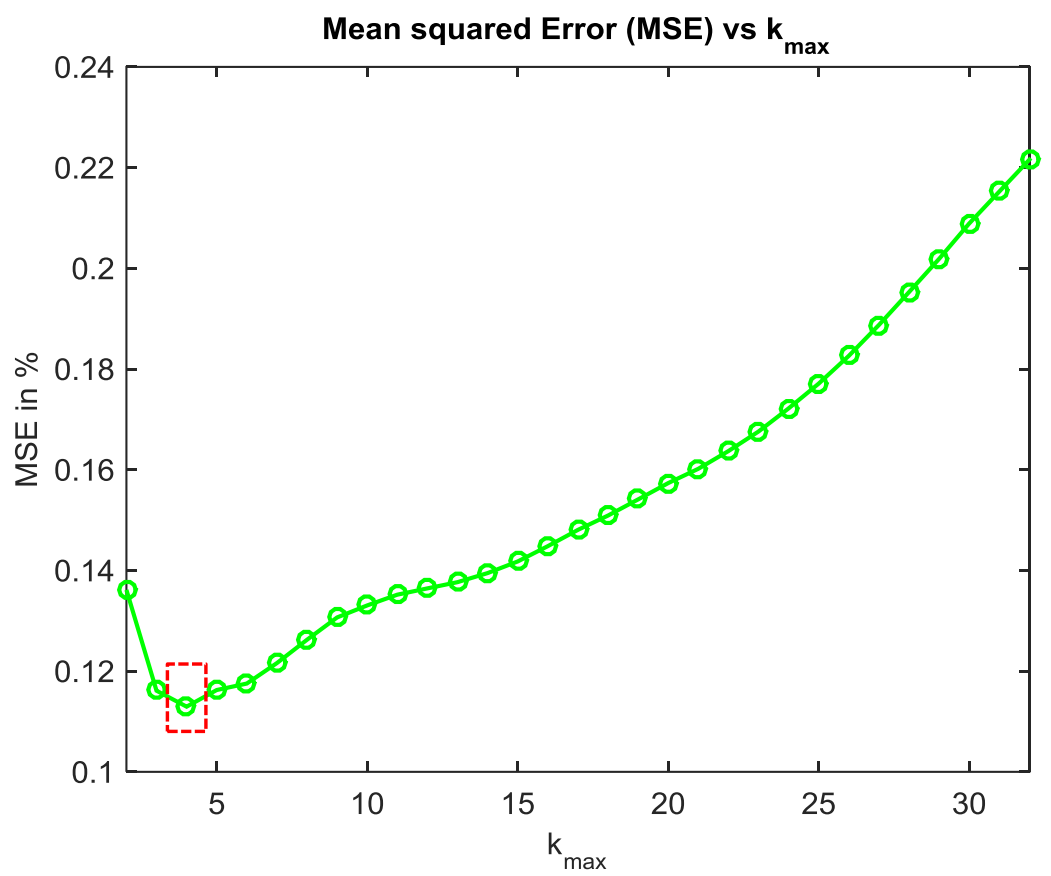

Figure 104. Computed MSE between the desired FD line and each column of $G_{\mathrm{H} \times \mathrm{k}_{\max }}$. 


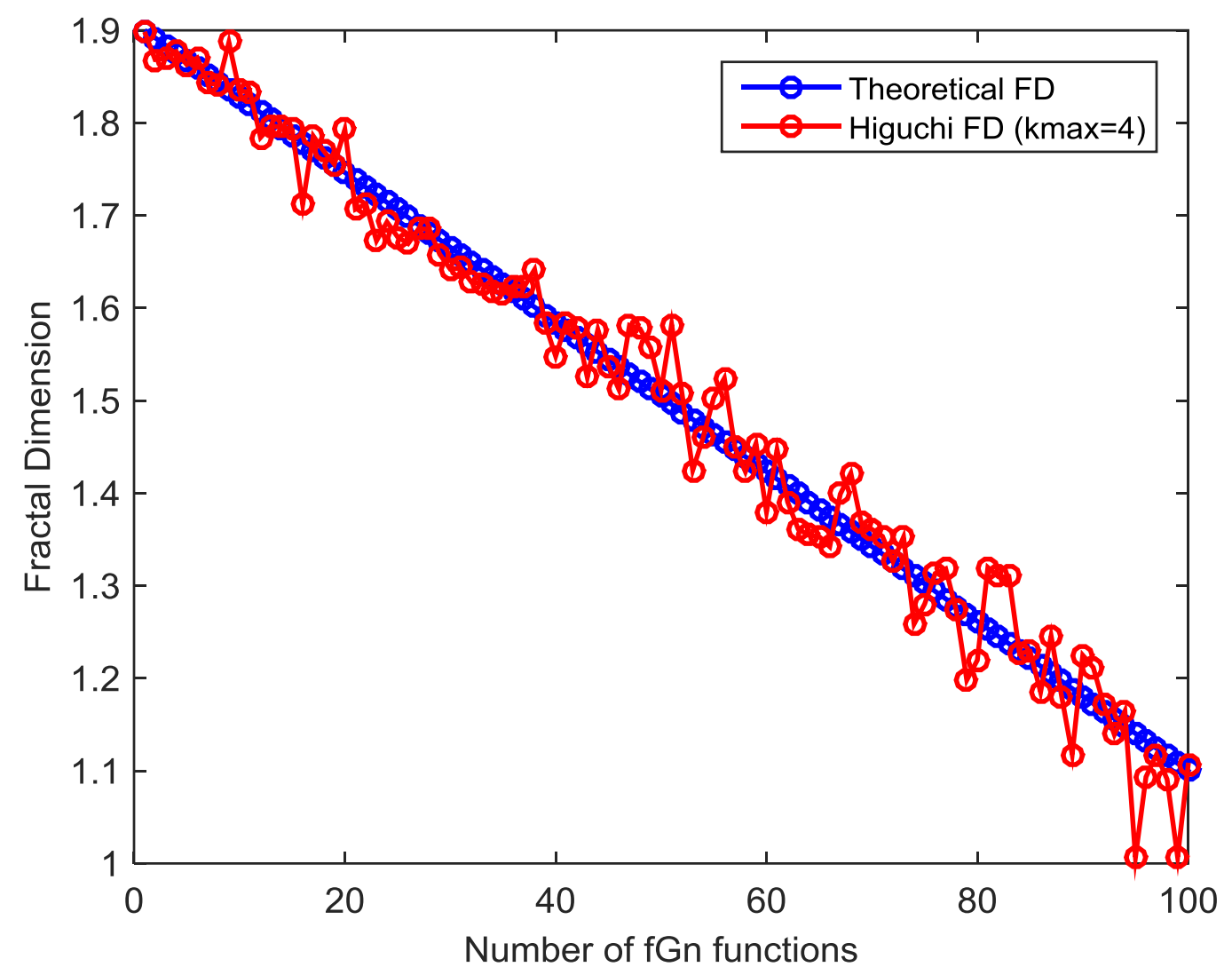

Figure 105. Plot of the theoretical FD line for the fGn set versus the best line computed via HFD for $\mathrm{k}_{\max }=4$.

Given the fact that the fGn signals are random ones, Figure 106 shows the best $\mathrm{k}_{\max }$ obtained after 1000 realizations of this same search experiment for 1000 different realizations of the fGn $s_{i}$ signal set. Figure 106 is a histogram of how many times a $\mathrm{k}_{\max }$ value appears along all the realizations performed on the various $s_{i}$ sets. The value that appeared most of the time was $\mathrm{k}_{\max }=4$, this value was the best candidate to grant the best FD estimate. It can be seen that as soon as $\mathrm{k}_{\max }$ starts to increase beyond 6 , the performance of the HFD to provide good FD estimates diminishes dramatically, to the point where $\mathrm{k}_{\max }$ values greater than 11 never show up in the histogram. Then, $\mathrm{k}_{\max }=4$ is the optimal free HFD parameter value $\approx 40 \%$ of the time. 


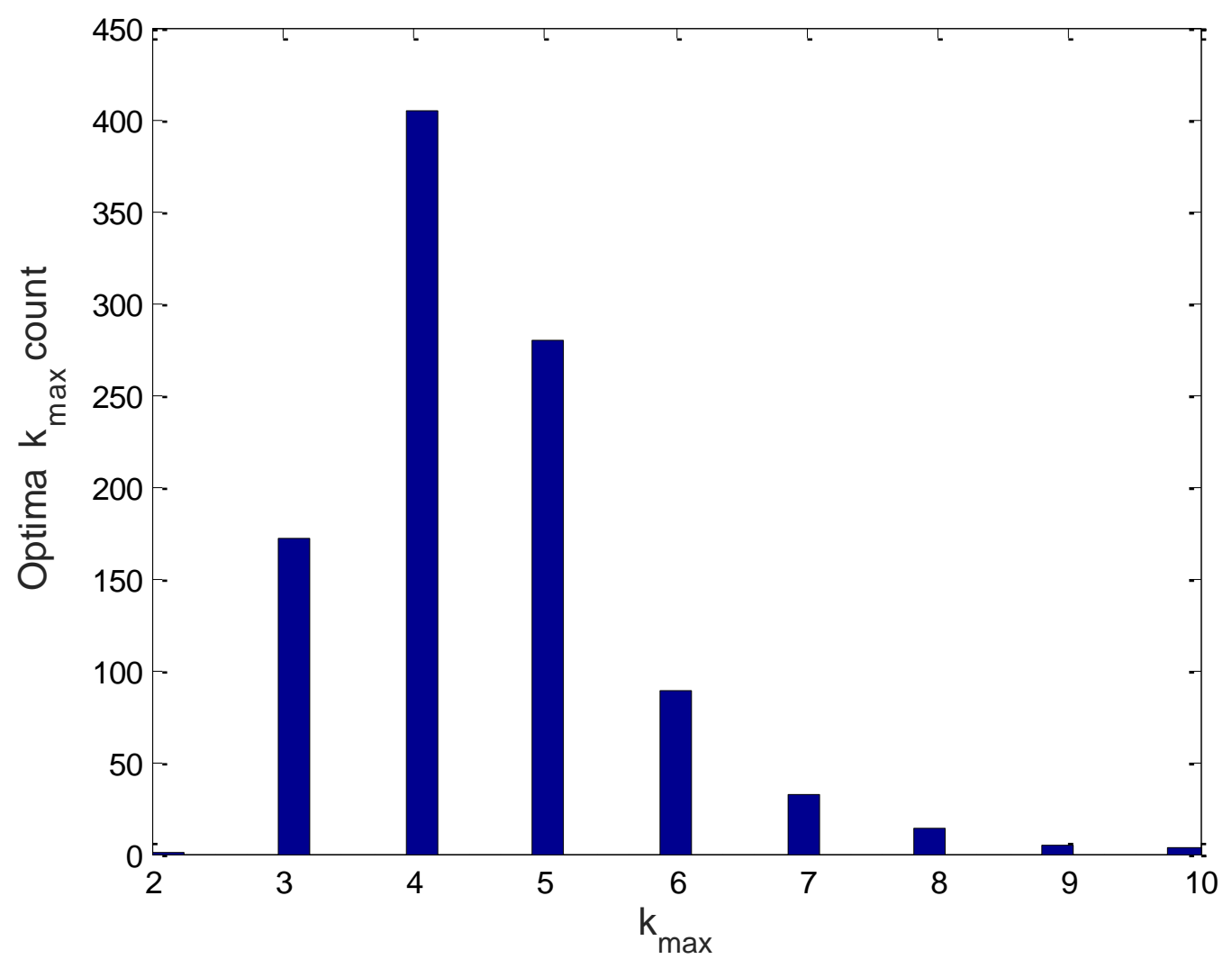

Figure 106. Histogram of the $\mathrm{k}_{\max }$ (the best one for each realization of a fGn set $s_{i}$ ) count for 1000 realizations of the experiment performed above with the various fGn $s_{i}$ sets, to infer their optimal $\mathrm{k}_{\max }$ value for HFD estimation.

Table 15 presents the HFD estimates for the fGn signals shown in Figure 102 for the found quasi-optimal free parameter value $\mathrm{k}_{\max }$. These studies of the HFD involving artificial signals granted the proper value of the free parameter $\mathrm{k}_{\max }$ to be used to analyze BWR real signals. This value is fixed at $\mathrm{k}_{\max }=4$ for all of the studied BWR signals. The decision behind the choosing of the used $\mathrm{k}_{\max }$ value (of 4) for BWR simulations lies in the observation that the fGn paths are visually very complex and are also very difficult artificial signals to implement (and visually speaking, the fGn paths are much more complex than the studied BWR real signals) and for them, there is a $\approx 40 \%$ chance of hitting the jackpot when computing the HFD with $\mathrm{k}_{\max }=4$ for any fGn signal. Thus, our choosing of $\mathrm{k}_{\max }=4$ for BWR signal analysis is linked to the fact that this particular value provided the best HFD estimates of intricate fGn signal paths. 
Table 15. Computed HFD value for eight fGn signals.

\begin{tabular}{cc}
\hline $\begin{array}{c}\text { Theoretical FD values } \\
\text { for some fGn paths }\end{array}$ & $\begin{array}{c}\text { HFD estimate } \\
\text { for } \mathrm{k}_{\max }=4\end{array}$ \\
\hline 1.9 & 1.8904 \\
1.8 & 1.7980 \\
1.7 & 1.5782 \\
1.6 & 1.4649 \\
1.5 & 1.4143 \\
1.4 & 1.3249 \\
1.3 & 1.2057 \\
\hline
\end{tabular}

\subsection{The HFD as a novel BWR stability indicator}

In this section, the HFD is tested with its free parameter $\mathrm{k}_{\max }$ fixed to 4 due to the fact that this value was the best possible $\mathrm{k}_{\max }$ with which to attain the best FD estimates for the studied fGn signals, which are very complicated signals to investigate (and far more complex than real BWR signals). Therefore, it is believed that $\mathrm{k}_{\max }=4$ will provide accurate FD estimates (hopefully the best ones) for real BWR signals. As a starting point, a simple detector methodology based on the HFD is given. Such detector methodology is tested with real signals that stem from a BWR. Our goal is to classify those signals into two categories: stable ones and unstable ones via analysis of signal irregularity or roughness. 


\subsubsection{A BWR instability detector methodology based on the HFD}

Now, a practical BWR detector methodology is introduced based on the HFD $\left(\mathrm{k}_{\max }=4\right)$ to infer whether a BWR signal is stable or unstable. Before giving the steps of this detector methodology. Let us define the next two estimators:

- HFD is a practical estimator of the fractal dimension (FD) for time series.

- $\mathrm{H}_{\mathrm{e}}$ is a practical estimator of the Hurst exponent $(\mathrm{H})$ for time series.

The relationship that links HFD and $\mathrm{H}_{\mathrm{e}}$ is the plug-in Eq. (6) which can be thought of as mapping of the HFD estimates from the interval [1,2] to the interval [0,1] which might be convenient in an online stability monitor for reasons that in the following will be given. However, this particular mapping of the HFD estimates to $\mathrm{H}_{\mathrm{e}}$ ones is optional and up to the BWR user in turn. This mapping is in fact a benefit of working with the HFD and it is of utter importance to highlight it).

The main steps of the methodology for novel non-linear indicator of BWR stability is given as follows:

1. The considered signal (APRM or LPRM) obtained from the BWR is segmented in windows of $10 \mathrm{~s}$ of duration.

2. Each segmented signal (APRM or LPRM) is studied with the HFD method, with the free parameter $\mathrm{k}_{\max }=4$ to estimate its HFD.

3. The mean (mean(HFD)) and standard deviation (std(HFD)) of the HFD estimates are calculated and averaged along all the studied segments of $10 \mathrm{~s}$.

4. Although the HFD is the main protagonist of this detector methodology. The Hurst exponent estimate $\left(\mathrm{H}_{\mathrm{e}}\right)$ can also be used to just range the HFD estimated values between 0 and 1 (plug in the HFD estimates in Eq. (6) to compute $H_{e}$ ). This step can be considered as optional.

A high HFD $(\approx 1.5)$ estimated value points out high irregularity and high unpredictability of the studied BWR signal (LPRM or APRM) and this high value might also indicate that the studied signal could be noise. This noise (could be white noise), from the BWR perspective is a sign of stable behavior. On the other hand, a low HFD $(\approx 1)$ estimated value points out high predictability and high regularity of the studied BWR signal, also, this low value might indicate that the studied signal could be a regular sinusoid waveform. This particular waveform, in the context of BWR signal analysis is a sign of BWR unstable (i.e., undesirable) behavior due to the presence of a density wave, which manifests in the LPRM or APRM signals as a sinusoid function with a natural frequency that oscillates around 0.5 Hz. If the Hurst exponent is employed (a reminder, the Hurst exponent is an optional indicator, but it is important to highlight it, because HFD estimates are related to $\mathrm{H}_{\mathrm{e}}$ via a linear relationship), a high HFD value (sign of stable BWR behavior) is mapped to a low $\mathrm{H}_{\mathrm{e}}$ value close to 0.5 whereas a low HFD value (sign of unstable BWR behavior) is 
mapped to a high $\mathrm{H}_{\mathrm{e}}$ value close to 1 . Then, when HFD increases, the Hurst exponent decreases linearly.

\subsection{Results and discussions}

Now we validate the methodology given in Section 4.1 with the next data sets:

1. Data set from a typical BWR:

a) 207 steady state condition signals (i.e. stable signals).

b) 96 unstable signals.

2. Case 4 from the Forsmark stability benchmark:

a) 1 APRM signal plus 22 LPRM signals.

3. Case 9 cycle 14 of the Ringhals stability benchmark:

a) 72 LPRM signals.

\subsubsection{Stable signals}

The aim of this test is to validate the behavior of the BWR in steady-state conditions. The results obtained are presented in the Figures 107-112. Figure 107 shows the plot of one APRM stable signal of a typical BWR.

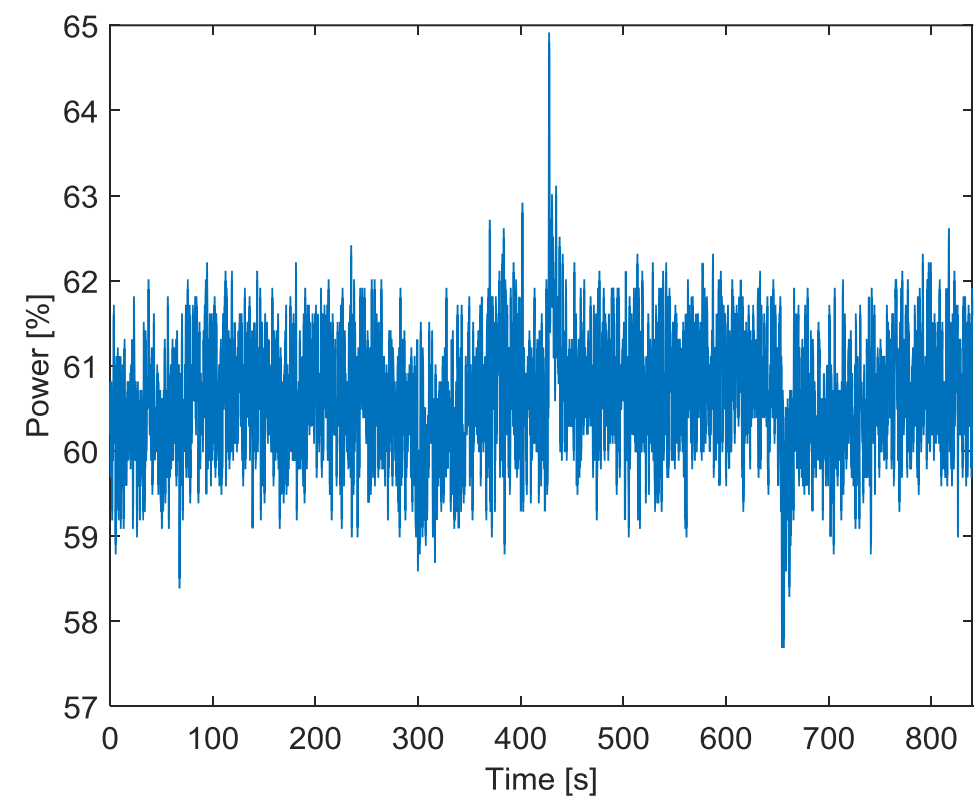

Figure 107. Stable signal from a typical BWR. 
Figure 108 shows in red color, a studied segment of $10 \mathrm{~s}$ of duration of the APRM signal, the FD of the red segment is estimated via HFD $\left(k_{\max }=4\right)$. The FD of the segment is 1.6431, such high FD value indicates that the studied signal is only acquisition noise around the steady state power signal, so the studied segment is stable and far from a sinusoid shape (unstable BWR signals have the shape of a quasi-sinusoid function).
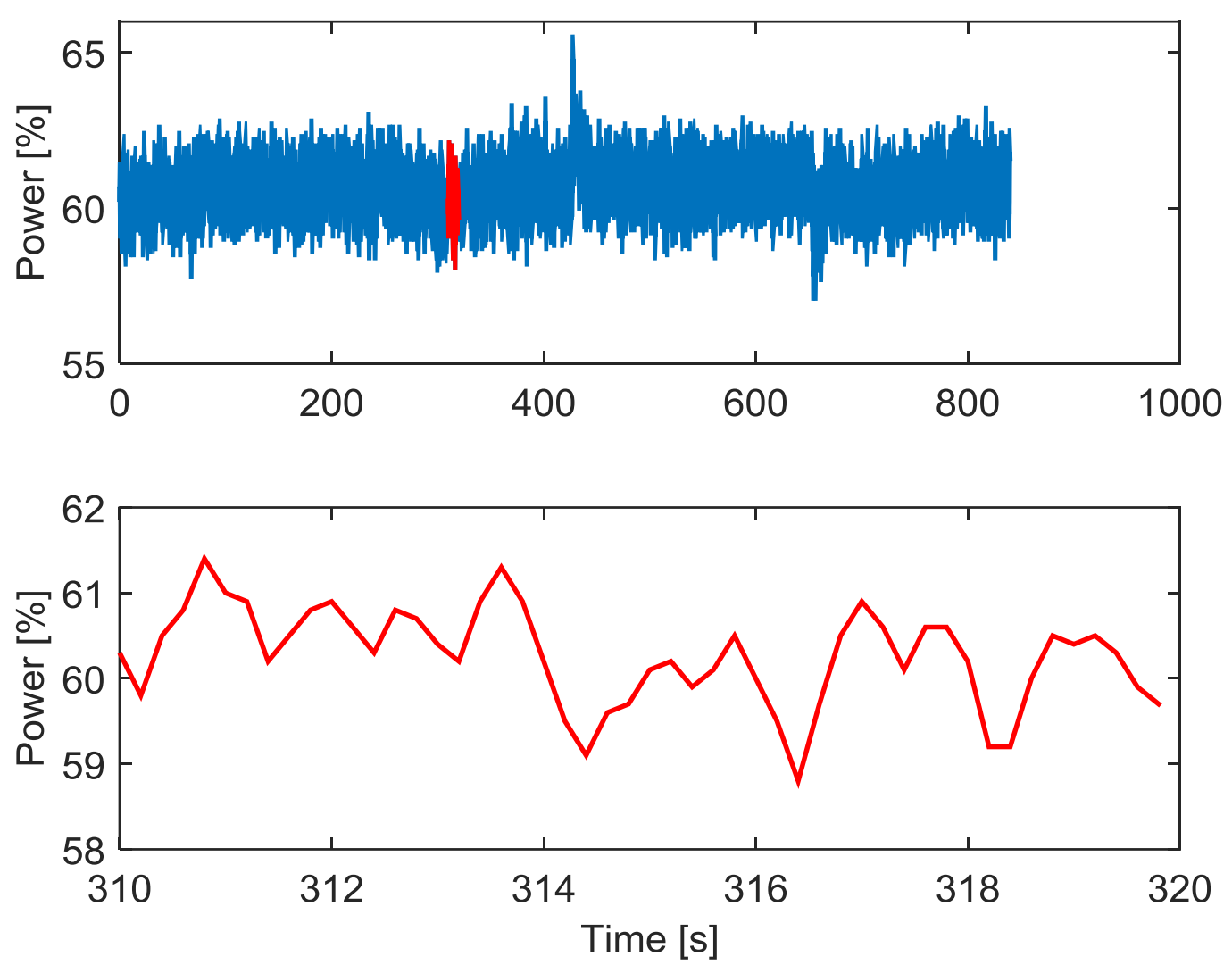

Figure 108. Analyzed segment of the studied APRM signal.

Figure 109 shows the estimated HFD along time for the studied APRM signal. The HFD oscillates very close to the high value of 1.5 and thus nothing unusual is going on in the core. Figure 110 shows the Hurst exponent estimate $\mathrm{H}_{\mathrm{e}}$ computed via HFD by the linear relation given by Eq. (6). It is observed that the value of $\mathrm{H}_{\mathrm{e}}$ along time oscillates very close to 0.5. The Hurst exponent value associated to white Gaussian noise. So, $\mathrm{H}_{\mathrm{e}}$ points to a typical stable case. The mean and the standard deviation are also computed in the final step. 


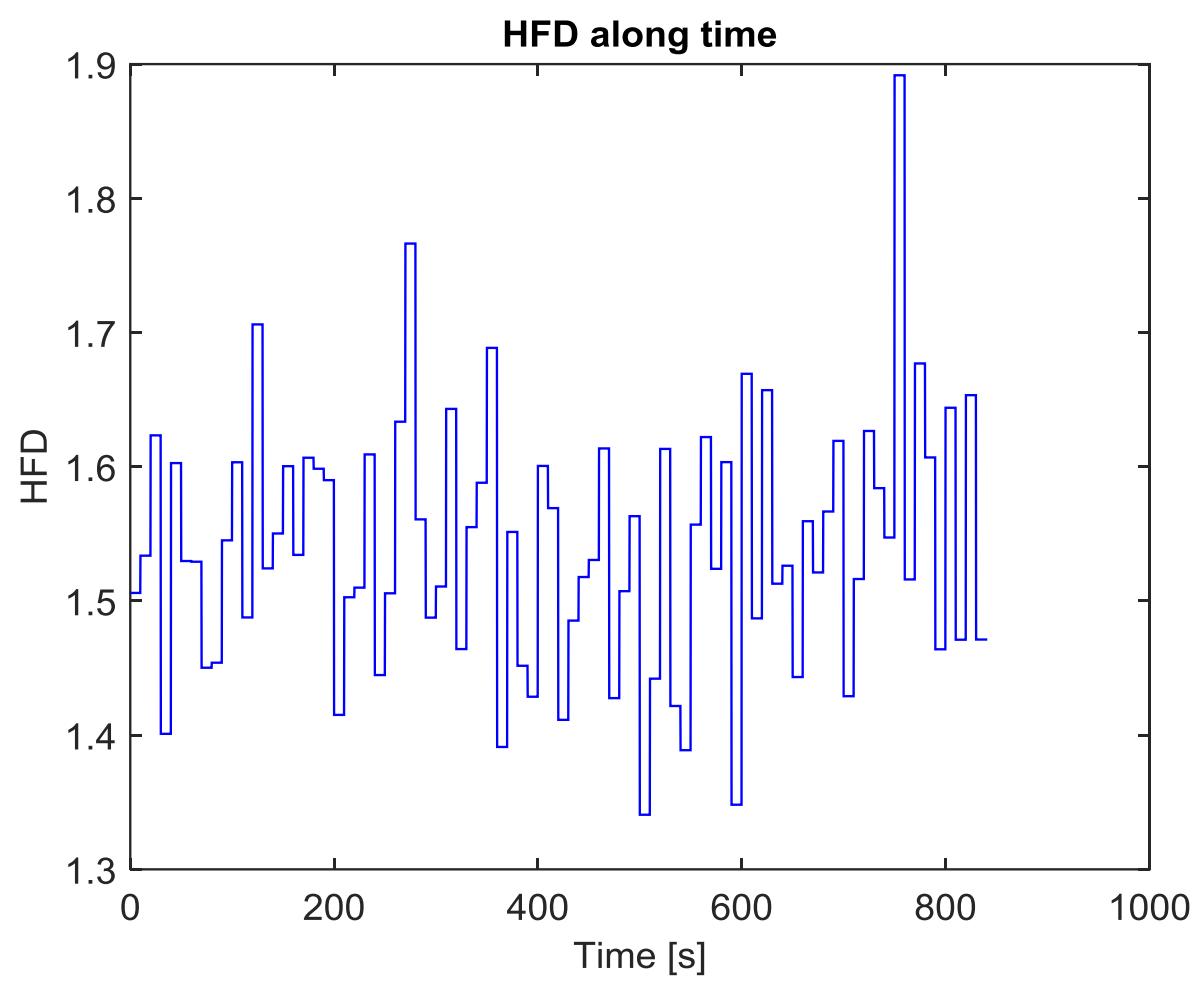

Figure 109. Estimated HFD along time for the studied stable APRM signal.

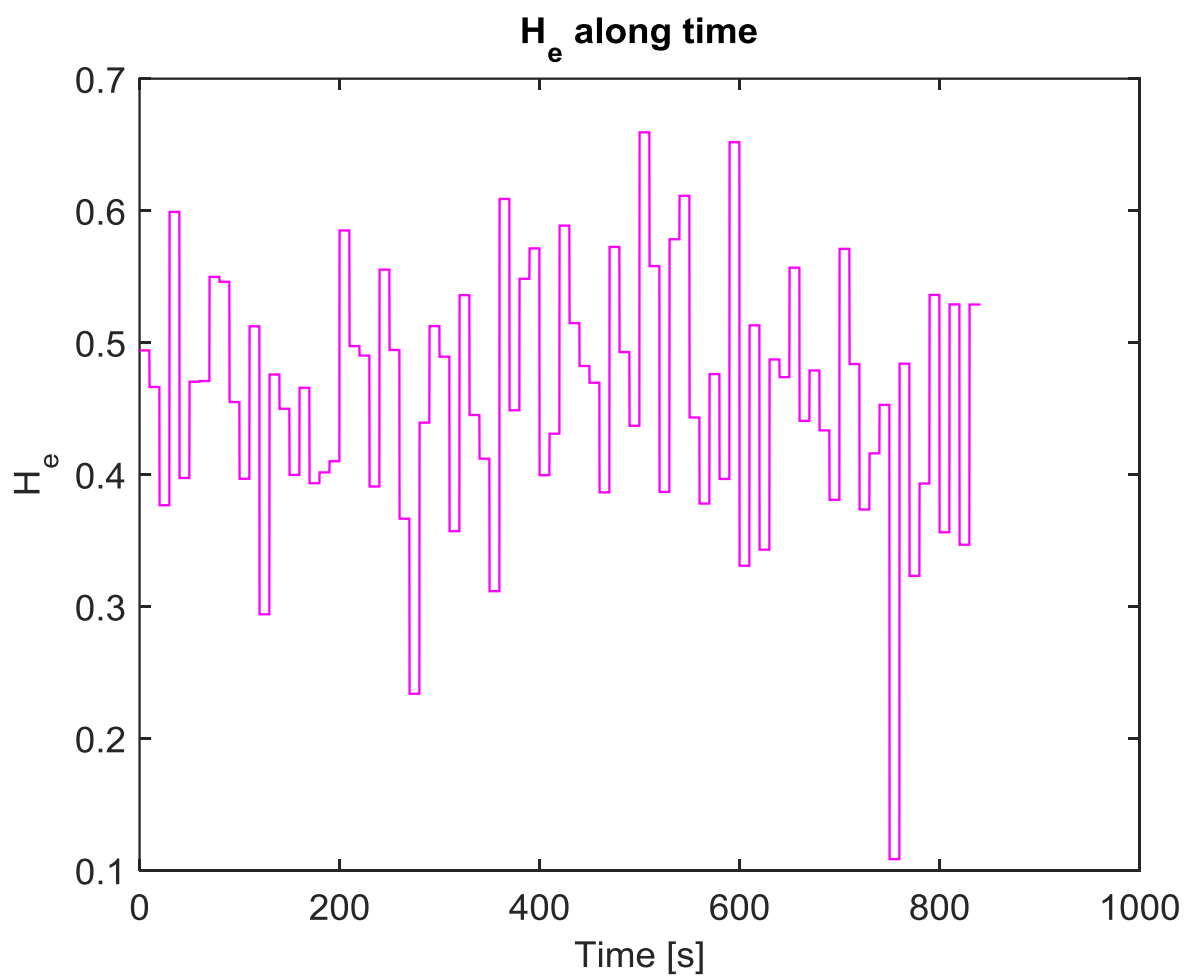

Figure 110. Estimated $\mathrm{H}_{\mathrm{e}}$ along time for the studied stable APRM signal. 
Table 16 shows the mean(HFD) computed along time and the std(HFD) of the studied APRM signal. Figure 111 shows a plot of the computed mean HFD estimates of the 207 signals (each HFD estimate is a blue bullet point). Figure 112 shows the computation of the mean value of $\mathrm{H}_{\mathrm{e}}$ for the entire stable signal set. A pattern appears, most of the mean $\mathrm{H}_{\mathrm{e}}$ estimates for the studied signal set oscillate very close to 0.5 . Thus, the mean $\mathrm{H}_{\mathrm{e}}$ values pinpoint to complex (i.e., rough) signals such as white Gaussian noise. Through HFD $\left(\mathrm{k}_{\max }=4\right)$ estimation, the proposed methodology is able to detect stable signals that are visually noisy ones.

Table 16. Mean(HFD) and std(HFD) of the analyzed APRM

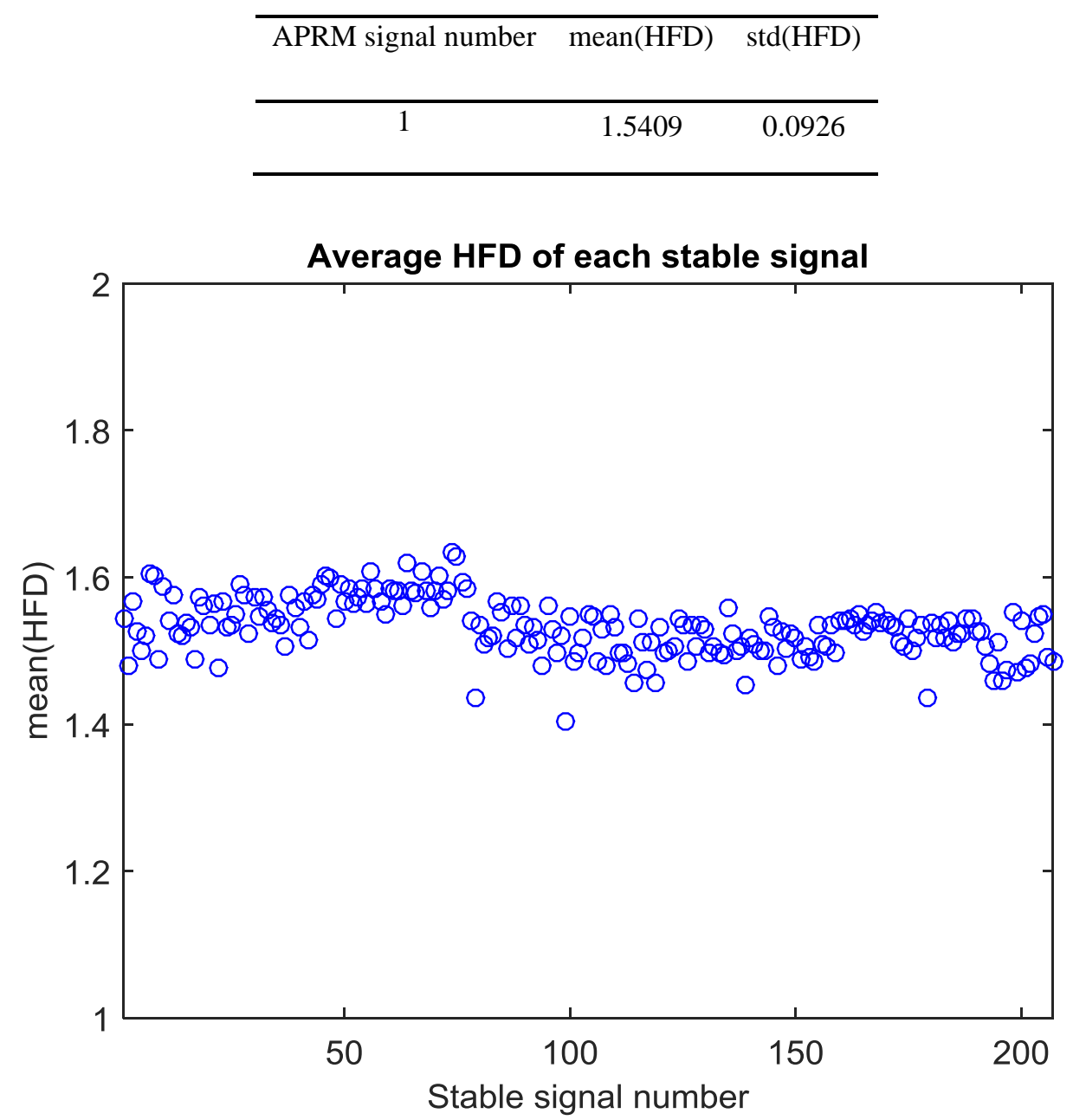

Figure 111. Computed mean (HFD) of the studied 207 stable signals. 


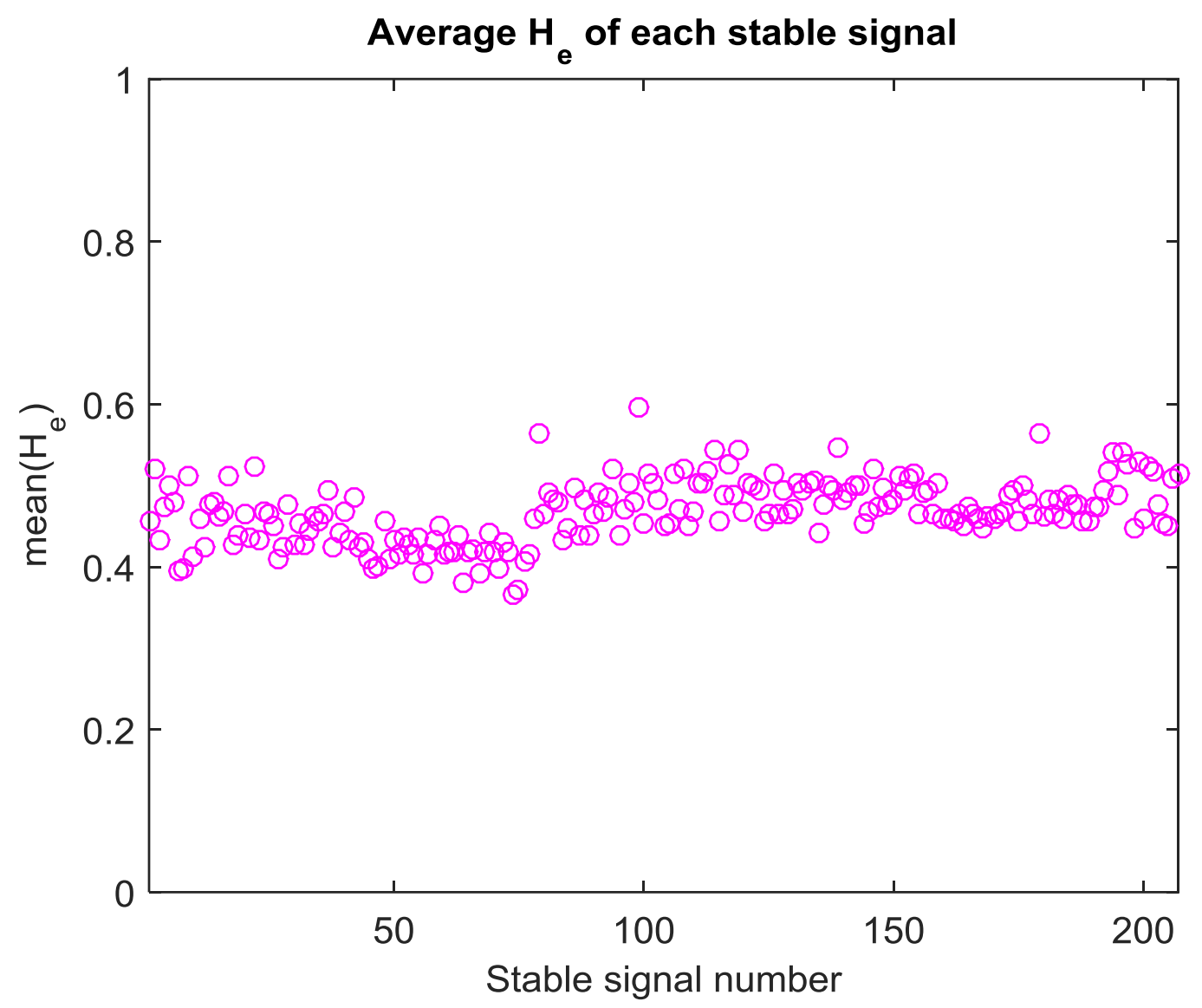

Figure 112. Computed mean $\left(\mathrm{H}_{\mathrm{e}}\right)$ of the studied 207 stable signals.

\subsubsection{Unstable signals of the laguna verde reactor}

The studied signal in this subsection stems from an instability event. This instability case consists of a total of 96 LPRM signals. Again, for reasons of space, only the analysis of one signal is presented. Figure 113 shows the plot the studied signal (from LPRM 1), this signal looks visually far from white noise. Figure 114 shows a studied segment of $10 \mathrm{~s}$, the segment looks like a quasi-sinusoid function. Its HFD $\left(\mathrm{k}_{\max }=4\right)$ estimate is 1.0499, this HFD estimate is a clear indication of signal regularity and low complexity. Thus, the HFD points to a simple function, in this case a sinusoidal function. 


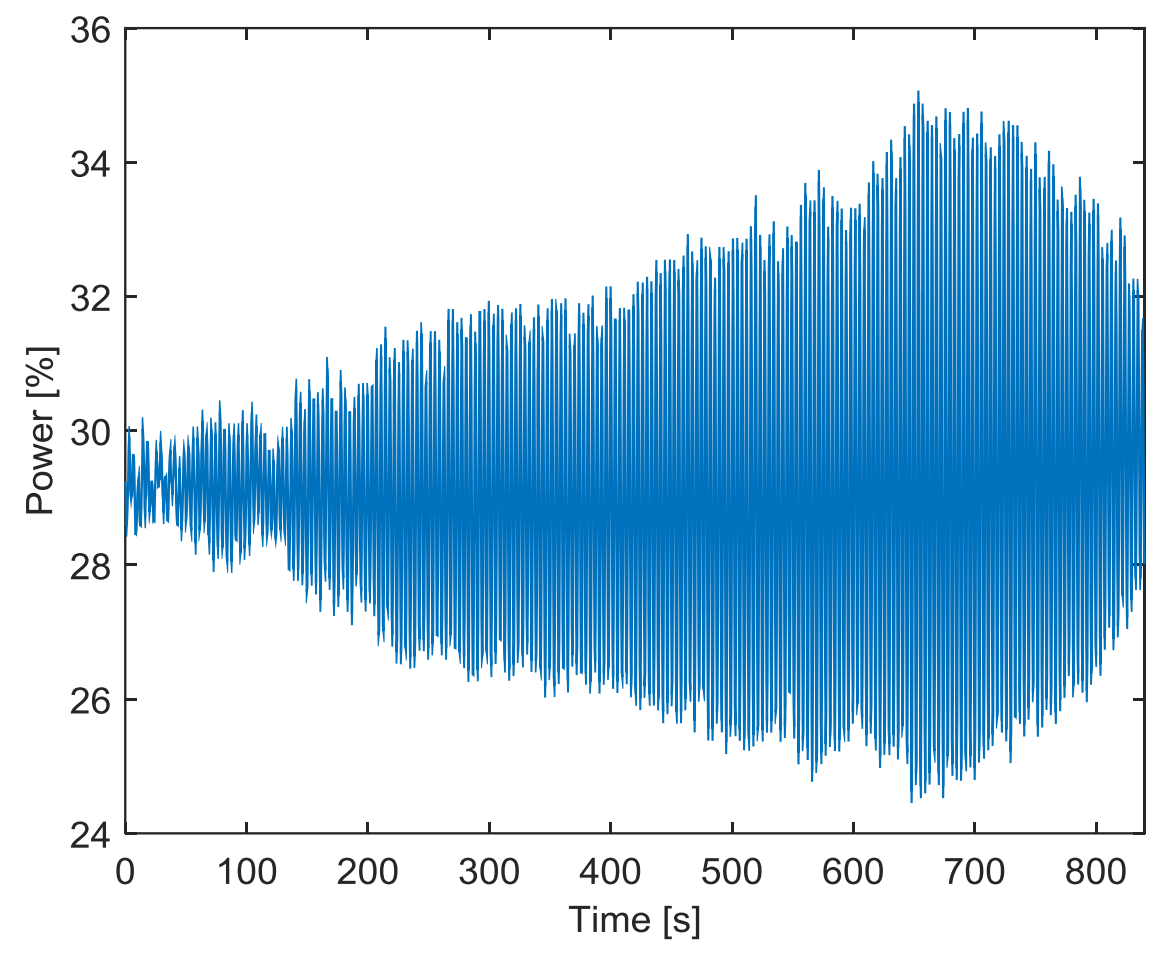

Figure 113. Unstable signal from the Laguna Verde reactor.
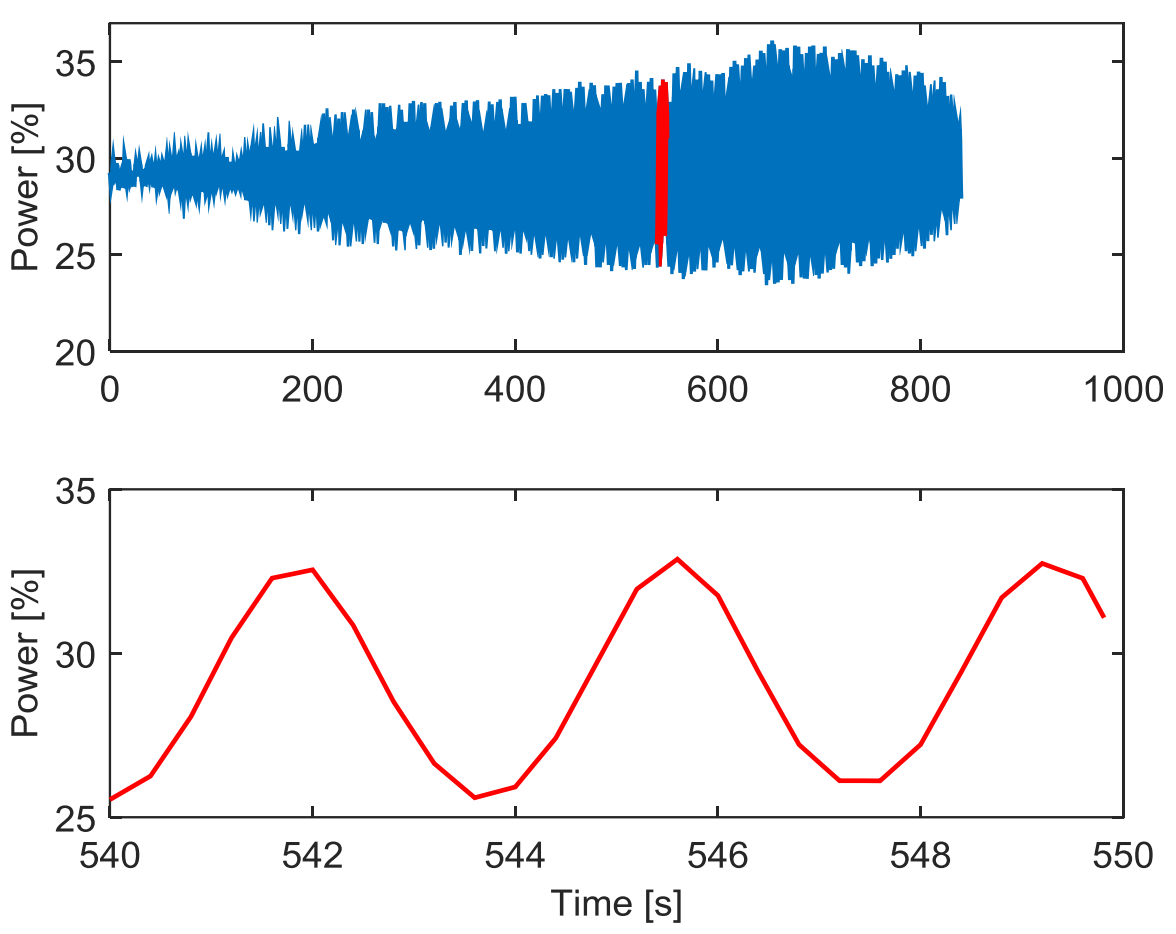

Figure 114. Analyzed segment of the studied LPRM 1 signal. 
Figure 115 shows the estimates of the HFD along time for the LPRM 1 signal. The HFD oscillates close to 1, pointing to high signal regularity (i.e., a quasi-sinusoidal function that has little to no roughness). Figure 116 shows the $\mathrm{H}_{\mathrm{e}}$ estimate of this LPRM 1. Its Hurst exponent $\mathrm{H}_{\mathrm{e}}$ is high and very close to 1 (and much higher than 0.9). $\mathrm{H}_{\mathrm{e}}$ is also pinpointing to low regularity and low complexity of the studied signal. In this case, $\mathrm{H}_{\mathrm{e}}$ points to a sinusoidal function linked to instability. Thus, the studied signal is unstable and the estimated indicator (HFD) also indicates that the LPRM 1 signal is far from desirable acquisition white noise that stems from stable BWR operation. Table 17 shows the computed mean(HFD) and the std(HFD) of the studied LPRM 1 signal.

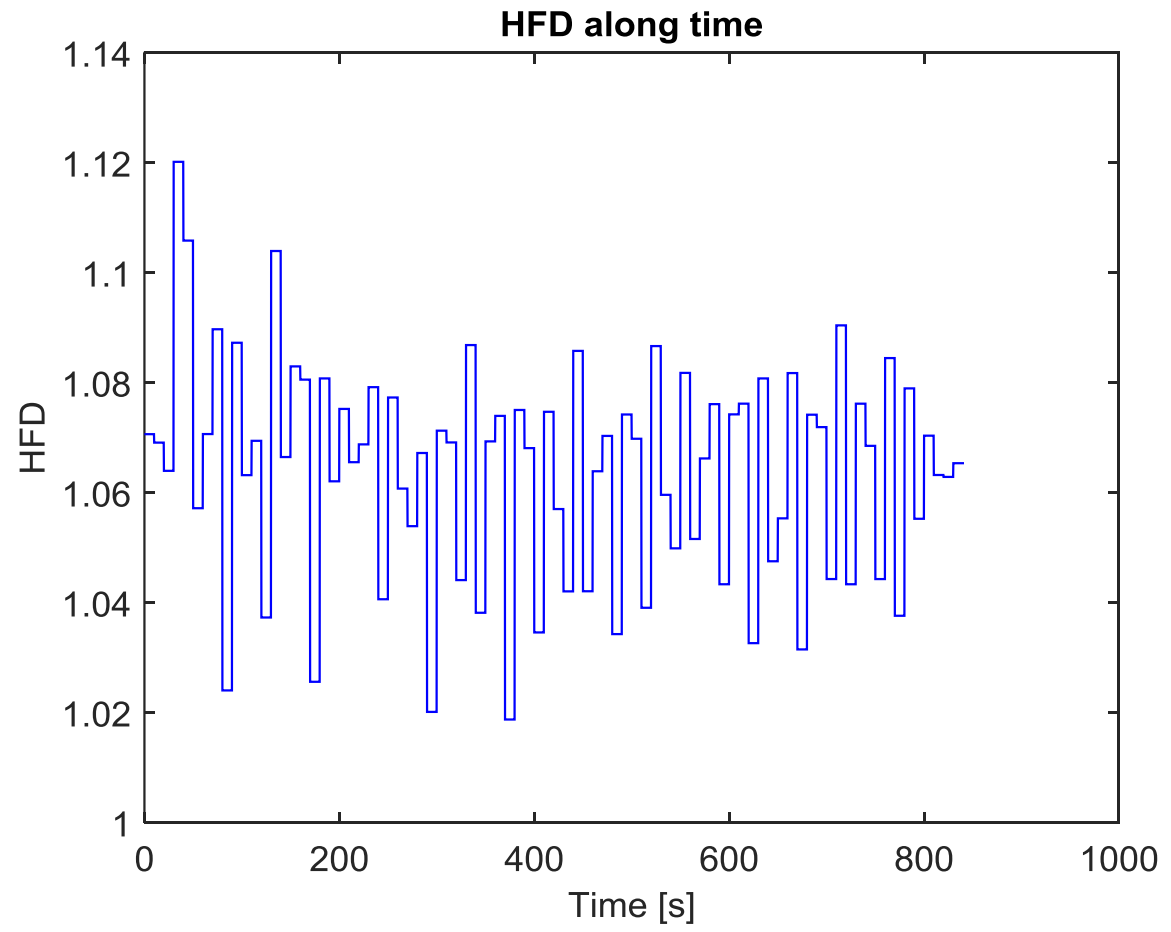

Figure 115. Estimated HFD along time for the studied unstable LPRM 1 signal. 


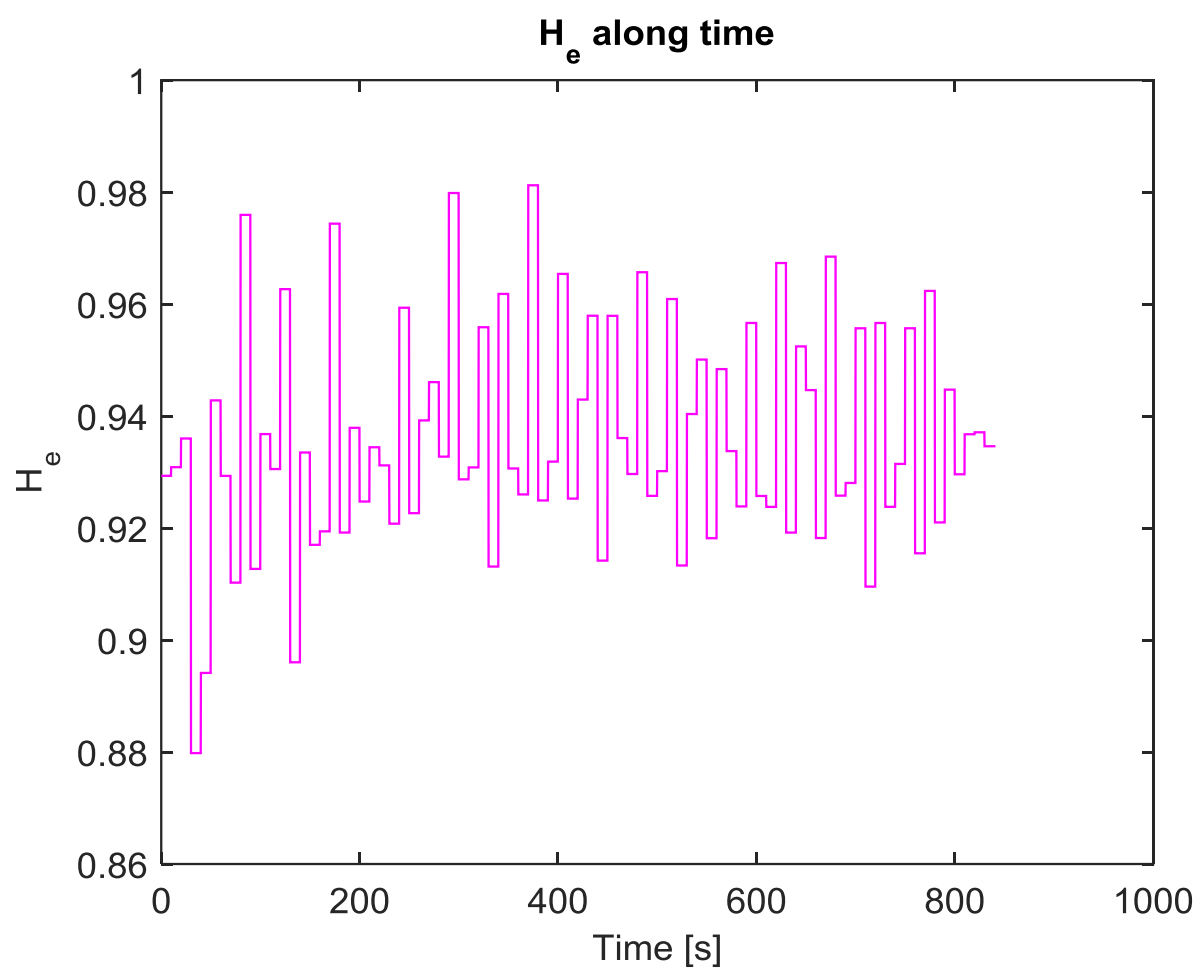

Figure 116. Estimated $\mathrm{H}_{\mathrm{e}}$ along time for the studied unstable LPRM 1 signal.

Table 17. Mean(HFD) and std(HFD) of the studied LPRM 1 signal.

\begin{tabular}{ccc}
\hline LPRM signal number & mean(HFD) & std(HFD) \\
\hline 1 & 1.0639 & 0.0197 \\
\hline
\end{tabular}

Figure 117 shows a plot of the computed mean HFD estimates of the 96 signals (each HFD estimate is a black bullet point). The mean HFD values (there is one HFD value for each signal, so, there are 96 HFD mean values) oscillate close to 1 . Figure 118 shows the computation of the mean value of $\mathrm{H}_{\mathrm{e}}$ for the entire unstable signal set. A pattern appears, most of the mean $\mathrm{H}_{\mathrm{e}}$ estimates for the studied signal set oscillate very close to 1 .

Thus, the mean $\mathrm{H}_{\mathrm{e}}$ values pinpoint to regular (i.e. no rough) signals such as sinusoidal functions (sinusoidal functions related to BWR instability). Through $\operatorname{HFD}\left(\mathrm{k}_{\max }=4\right)$ estimation, the proposed methodology is able to detect in this context, unstable BWR signals. 


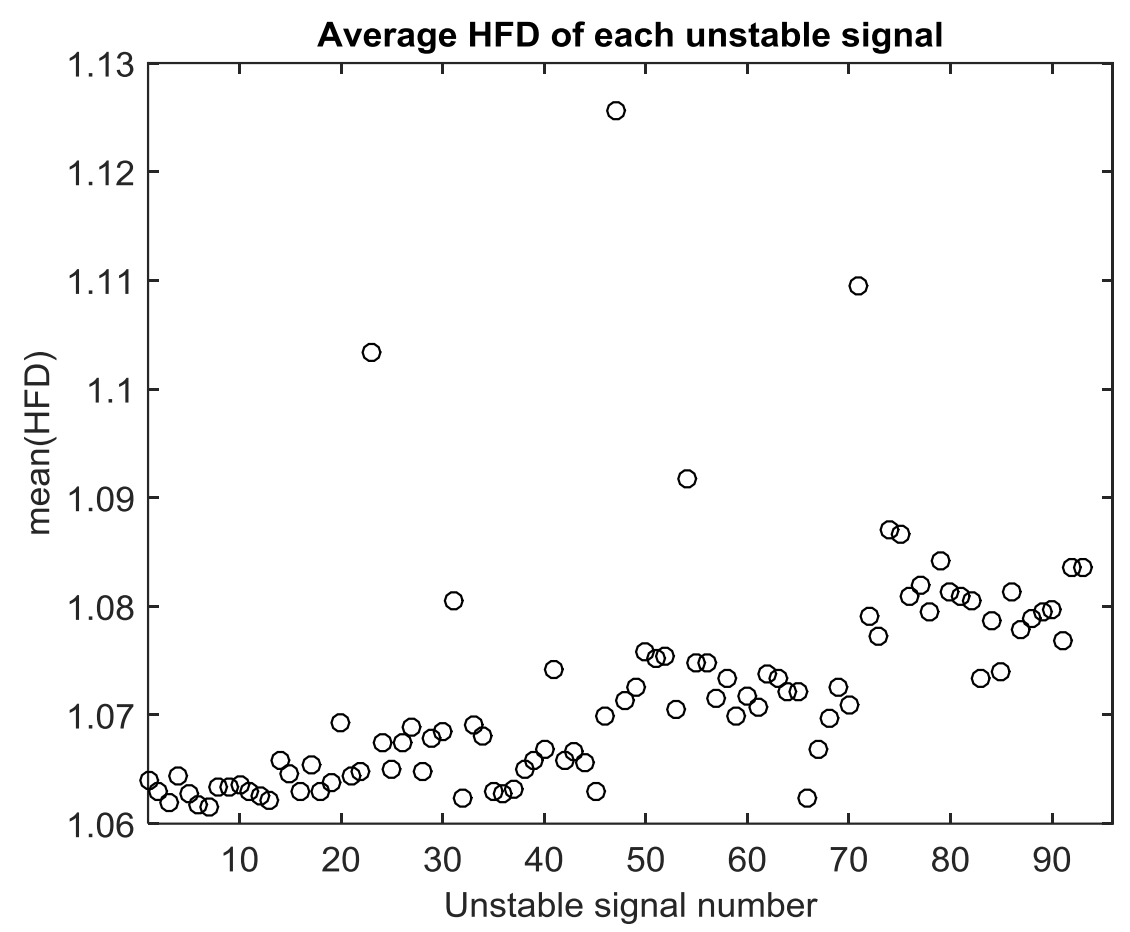

Figure 117. Computed mean (HFD) of the studied 96 unstable signals of the set.

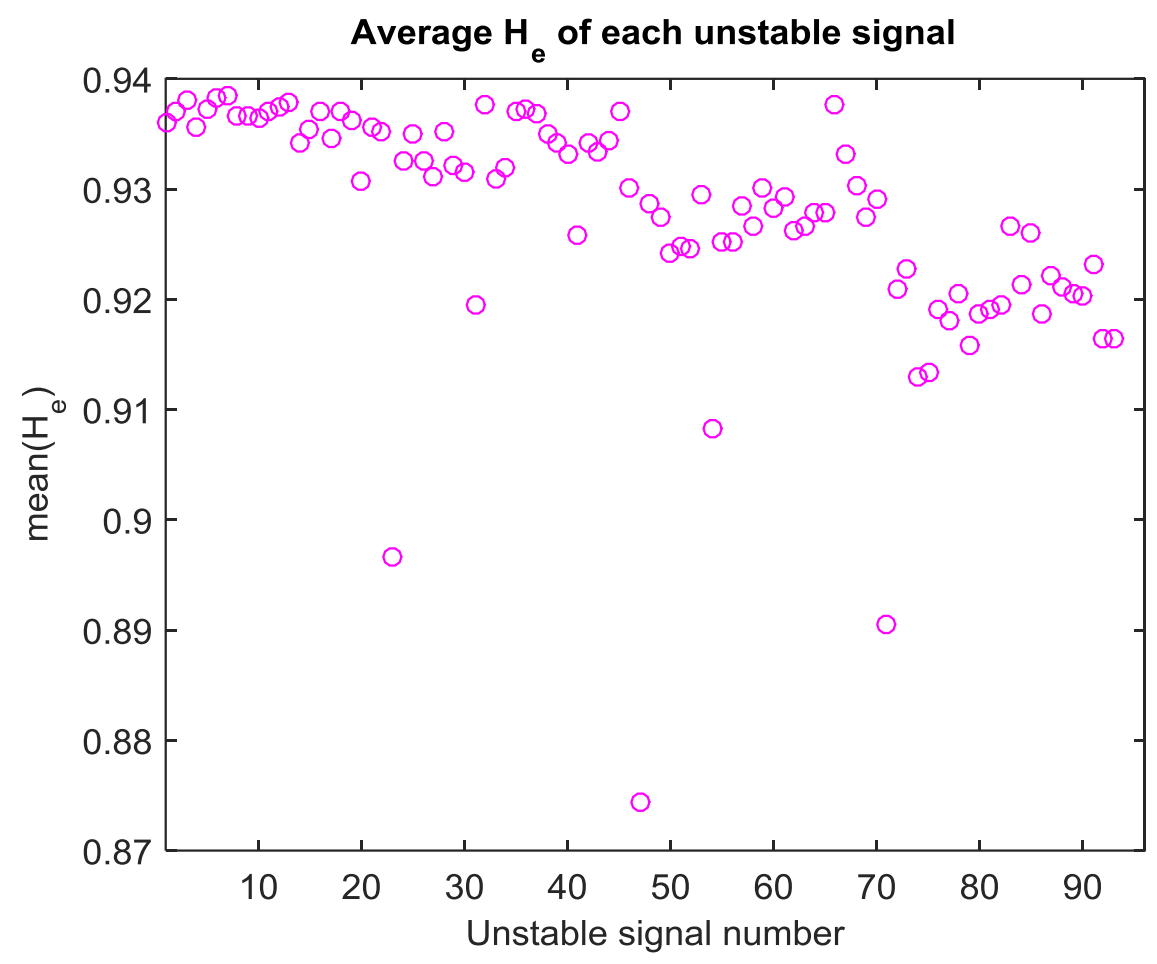

Figure 118. Computed mean $\left(\mathrm{H}_{\mathrm{e}}\right)$ of the studied 96 unstable signals of the set. 


\subsubsection{Comparison between stable and unstable states}

Finally, to complete the analysis of stable/unstable signals through HFD, Figure 119 shows a comparison of the mean HFD values of the studied sets (stable set of 207 stable signals plus a set of 96 unstable signals). The HFD mean values of the stable signals fluctuate very close to 1.5 whereas the HFD mean values of the unstable ones oscillate close to 1 . So, the HFD detects with success stable BWR signals from unstable ones via their complexity (i.e., roughness). Also, there is a distance of $\approx 0.5$ between the two states (stable from unstable). So, by fixing a HFD threshold value around 1.35 , it is possible to differentiate one state from the other properly. Any HFD estimate of a BWR signal segment higher than 1.35 is stable whereas a lower HFD value from this threshold is unstable.

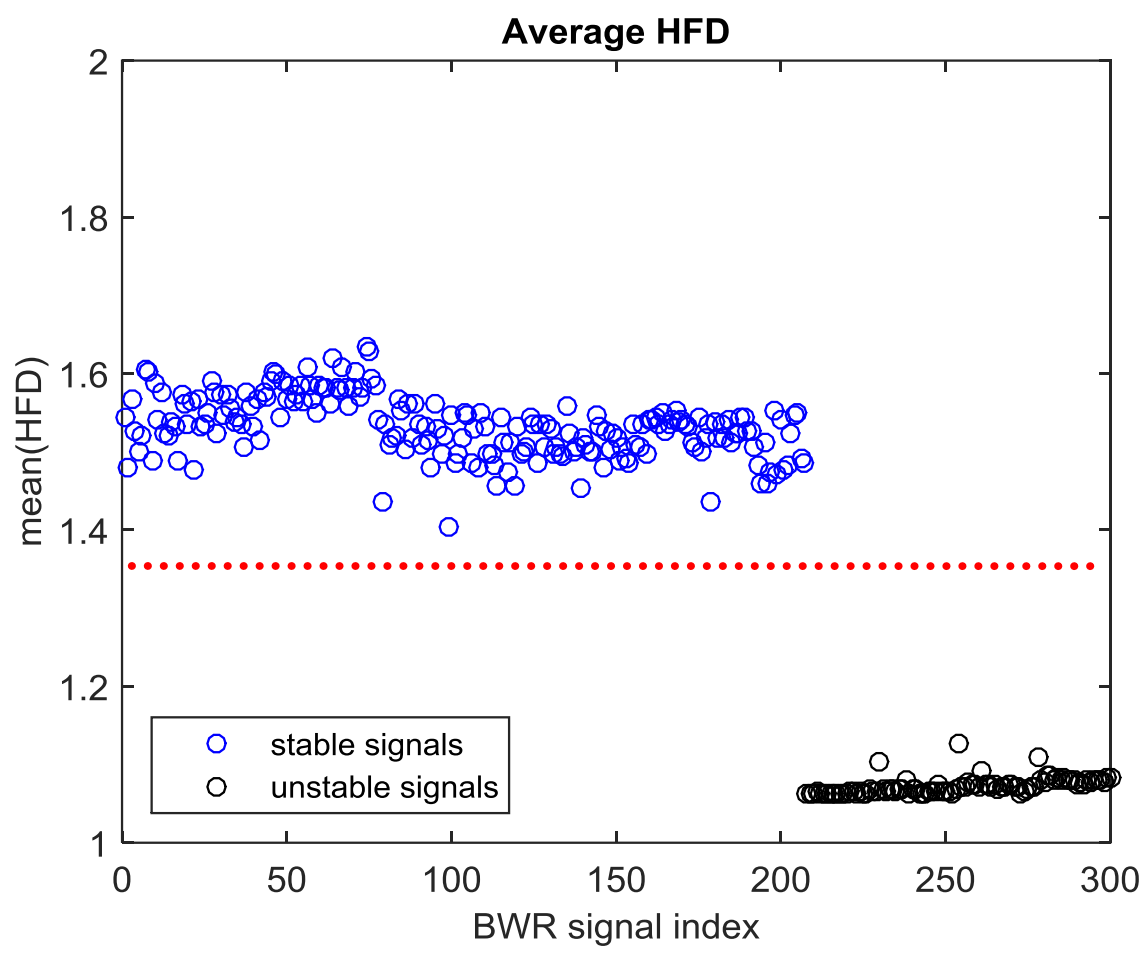

Figure 119. Comparison of the mean(HFD) values for both studied sets (a stable set of 207 APRM signals versus an unstable set of 96 LRPM signals).

Figure 120 shows comparison of the mean $\mathrm{H}_{\mathrm{e}}$ values of the studied sets (stable set of 207 signals plus unstable set of 96 signals). The $\mathrm{H}_{\mathrm{e}}$ mean values of the stable signals fluctuate very close to 0.5 whereas the $H_{e}$ mean values of the unstable ones oscillate close to 1 . So, the Hurst exponent $\mathrm{H}_{\mathrm{e}}$ detects with success stable BWR signals from unstable ones via their complexity (i.e., roughness). Also, there is a $\approx 0.5$ distance between the two states (stable from unstable). So, by fixing a $\mathrm{H}_{\mathrm{e}}$ threshold value around 0.65 . It is also possible to differentiate one state from the other properly via $\mathrm{H}_{e}$. Any $\mathrm{H}_{e}$ estimate of a BWR signal 
segment higher than 0.65 is unstable whereas a lower $\mathrm{H}_{\mathrm{e}}$ value from this threshold is a sign of stable behavior.

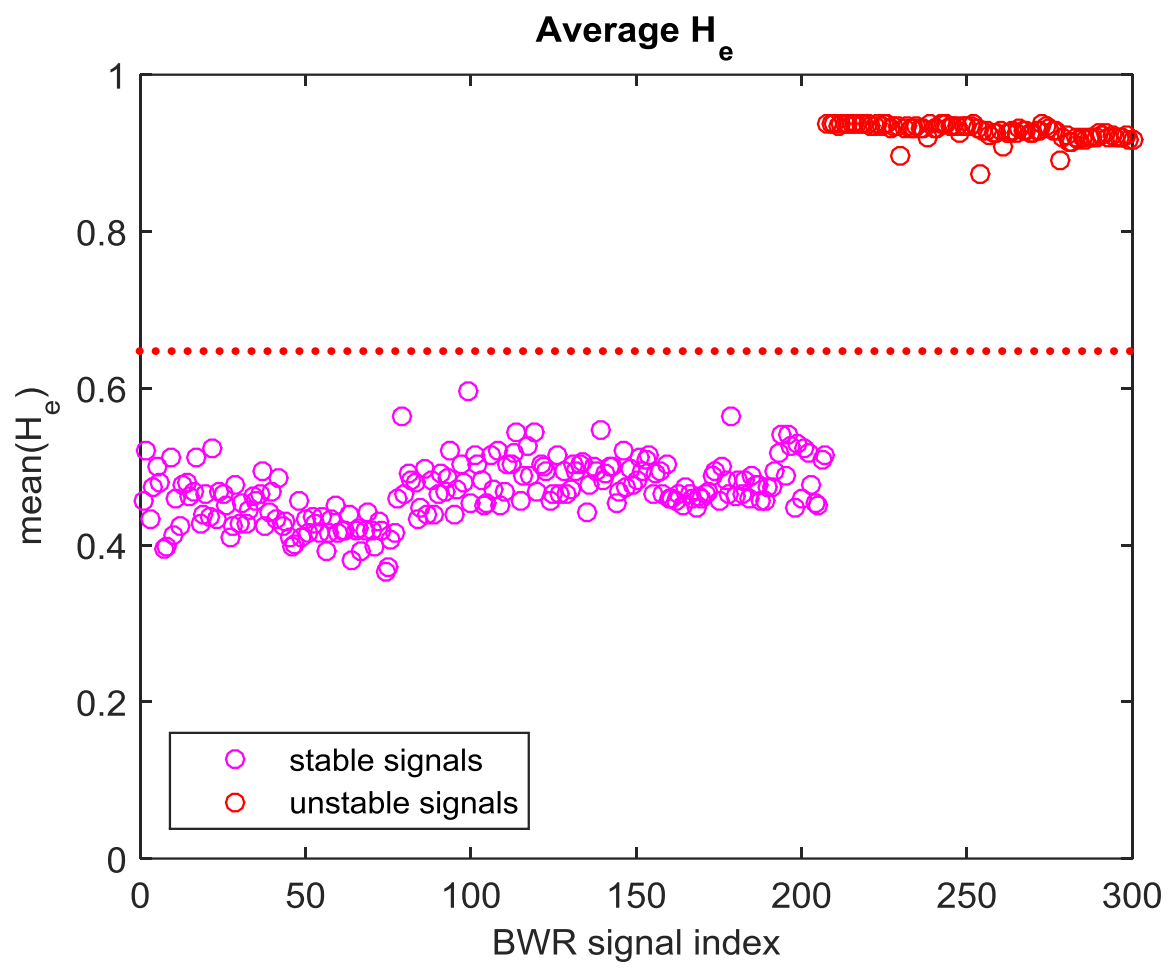

Figure 120. Comparison of the mean $\left(\mathrm{H}_{\mathrm{e}}\right)$ values for both studied sets (a stable set of 207 APRM signals versus an unstable set of 96 LRPM signals).

\subsubsection{Case 4 of the Forsmark stability benchmark}

Case 4 of the Forsmark stability benchmark (Verdú et al., 2001). This event is considered a challenging case to study by the complexity of the phenomenon. For reasons of space, only this case from this benchmark will be presented in a detailed way. This case contains a mixture between a global oscillation and a regional oscillation. This particular event corresponds to a situation where the neutronic power reactor suffers unusual unstable problems. The C4_APRM and C4_LPRM_x signals correspond to average power monitors (APRM) and local range monitors LPRM. The entire case 4 was studied that consists of a total of 23 signals, 22 LPRMs and 1 APRM. However, only the analysis of the APRM is presented in this work. The LPRMs analysis results is summarized in a Figure at the end of this section. Figure 121 shows the APRM signal. Figure 122 shows the plot of an analyzed $10 \mathrm{~s}$ studied segment, the HFD $\left(\mathrm{k}_{\max }=4\right)$ of the segment is 1.0556 , the HFD points to a regular smooth curve, in this context, a sinusoid like function was detected. Thus, the studied segment is unstable. Figure 123 shows the HFD estimates along time for this studied APRM signal. The HFD fluctuates very close to 1 with a few jumps above 1.2. The signal is unstable. Figure 124 shows the estimated $\mathrm{H}_{\mathrm{e}}$ along time, the estimated Hurst 
exponent values $\mathrm{H}_{\mathrm{e}}$ oscillate close to 1 . Thus, this complex APRM signal is overall unstable. Table 18 shows the mean(HFD) and the std(HFD) estimate of the studied APRM signal.

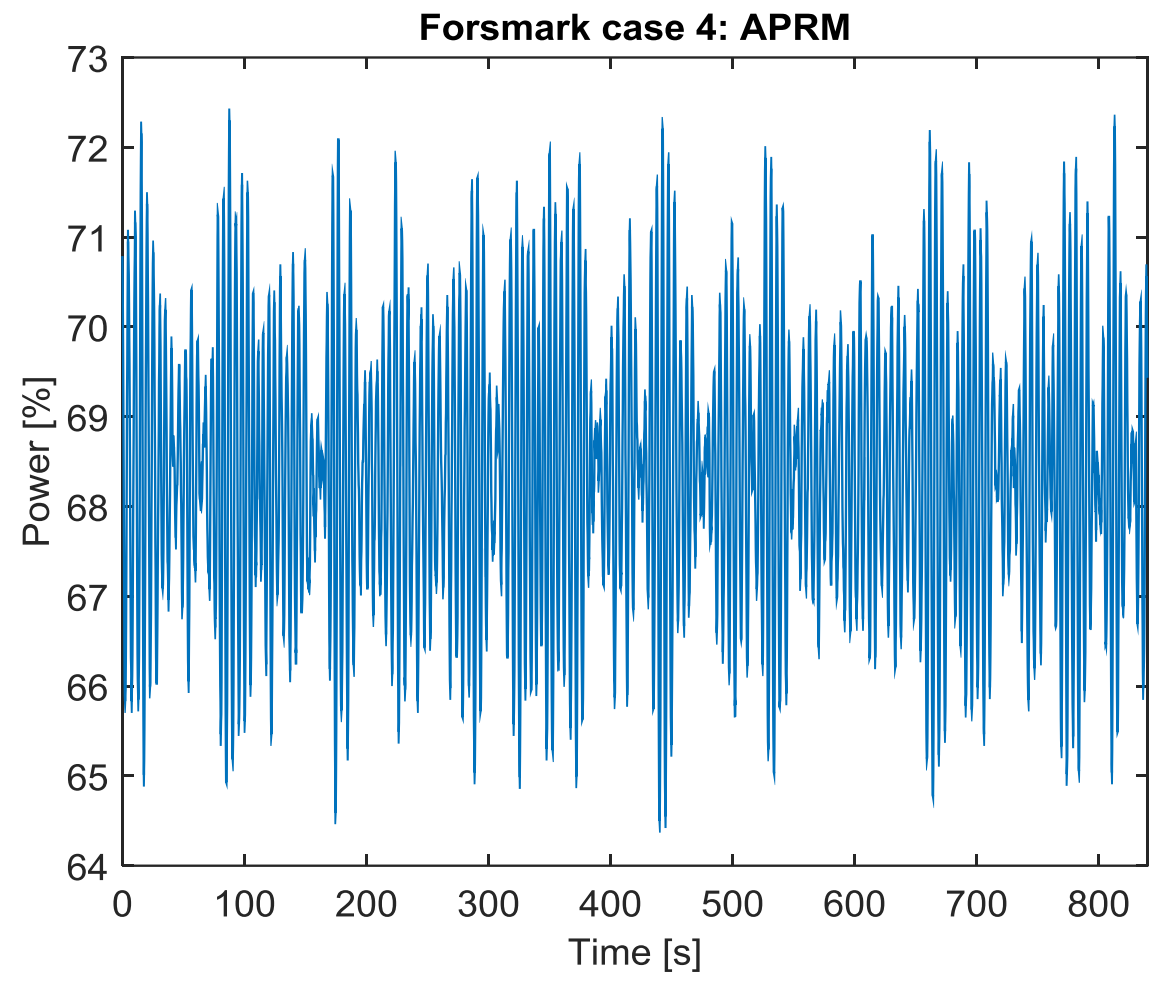

Figure 121. Forsmark case 4 APRM signal.
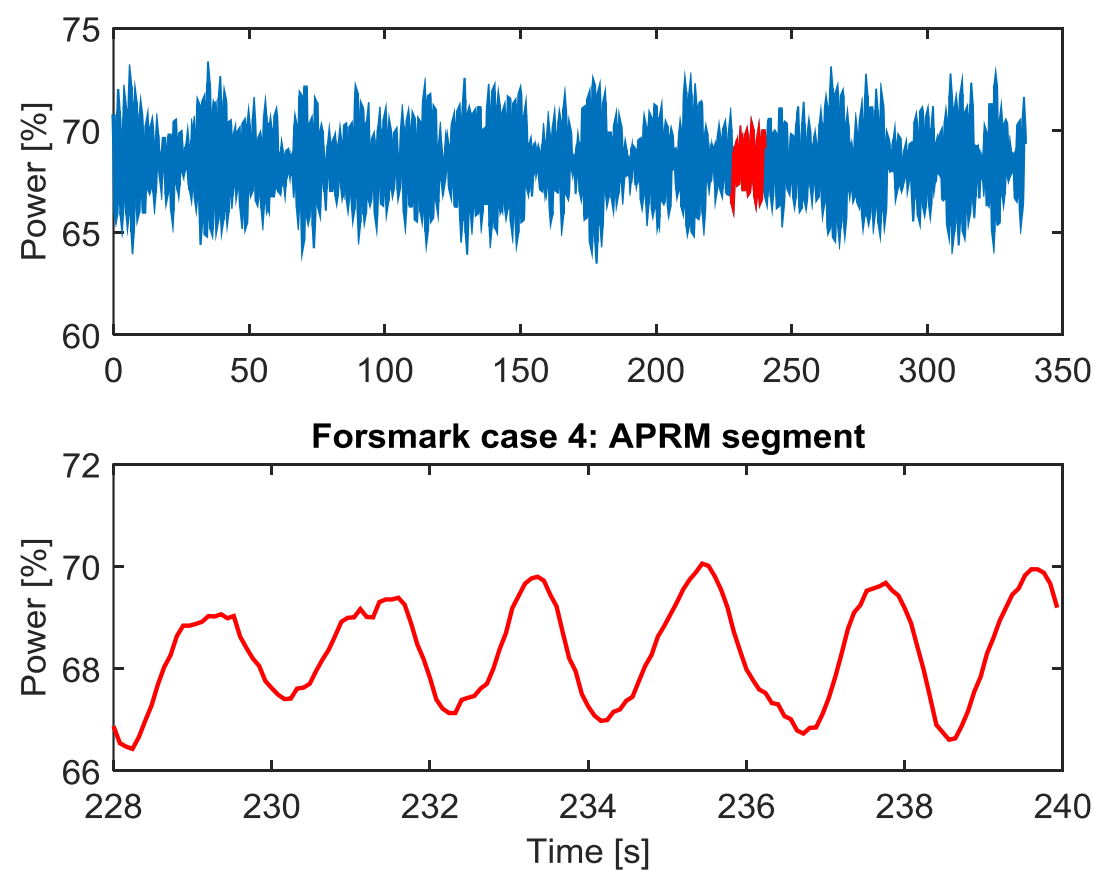

Figure 122. Studied segment of the Forsmark case 4 APRM. 


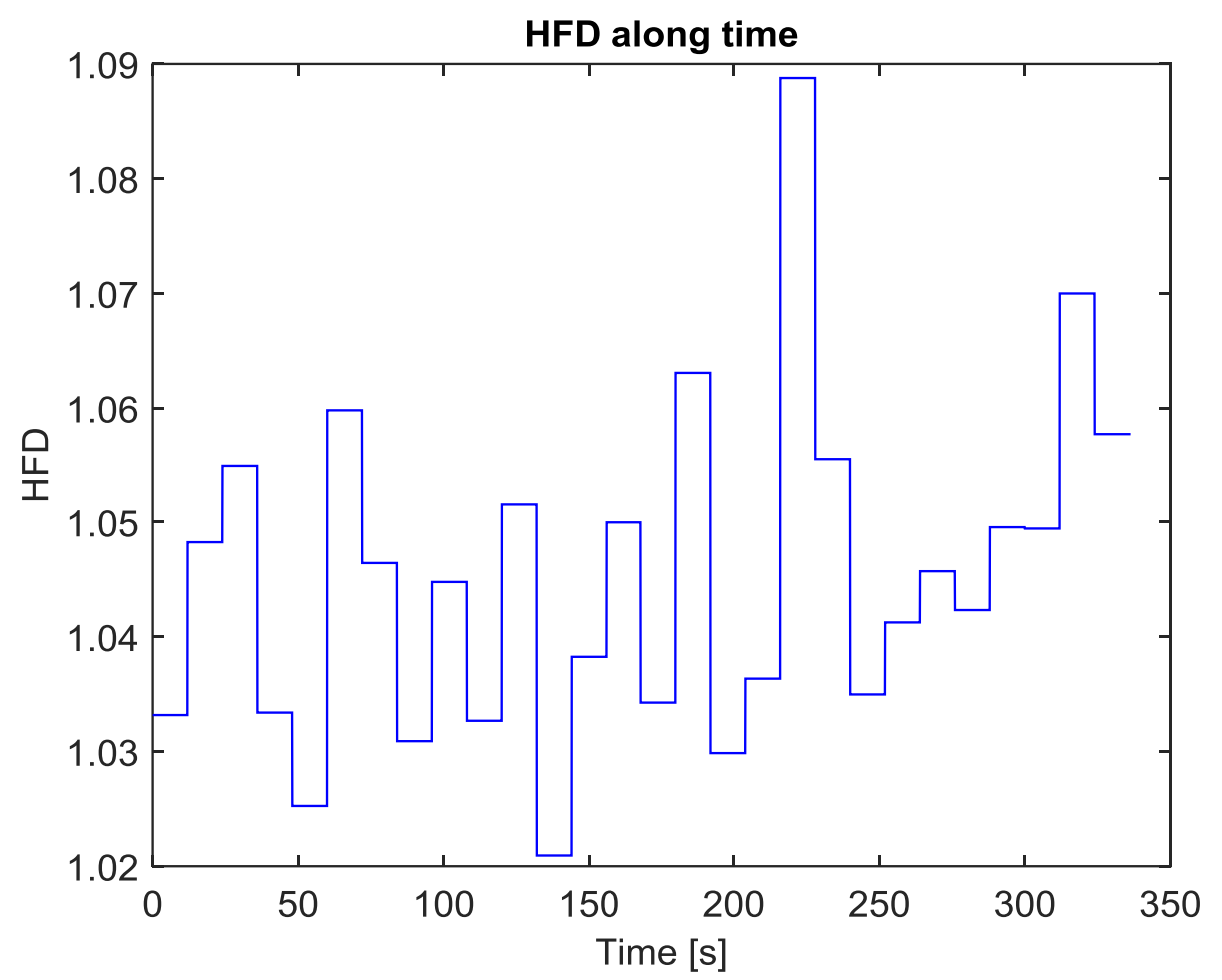

Figure 123. Estimated HFD along time for the Forsmark case 4 APRM signal.

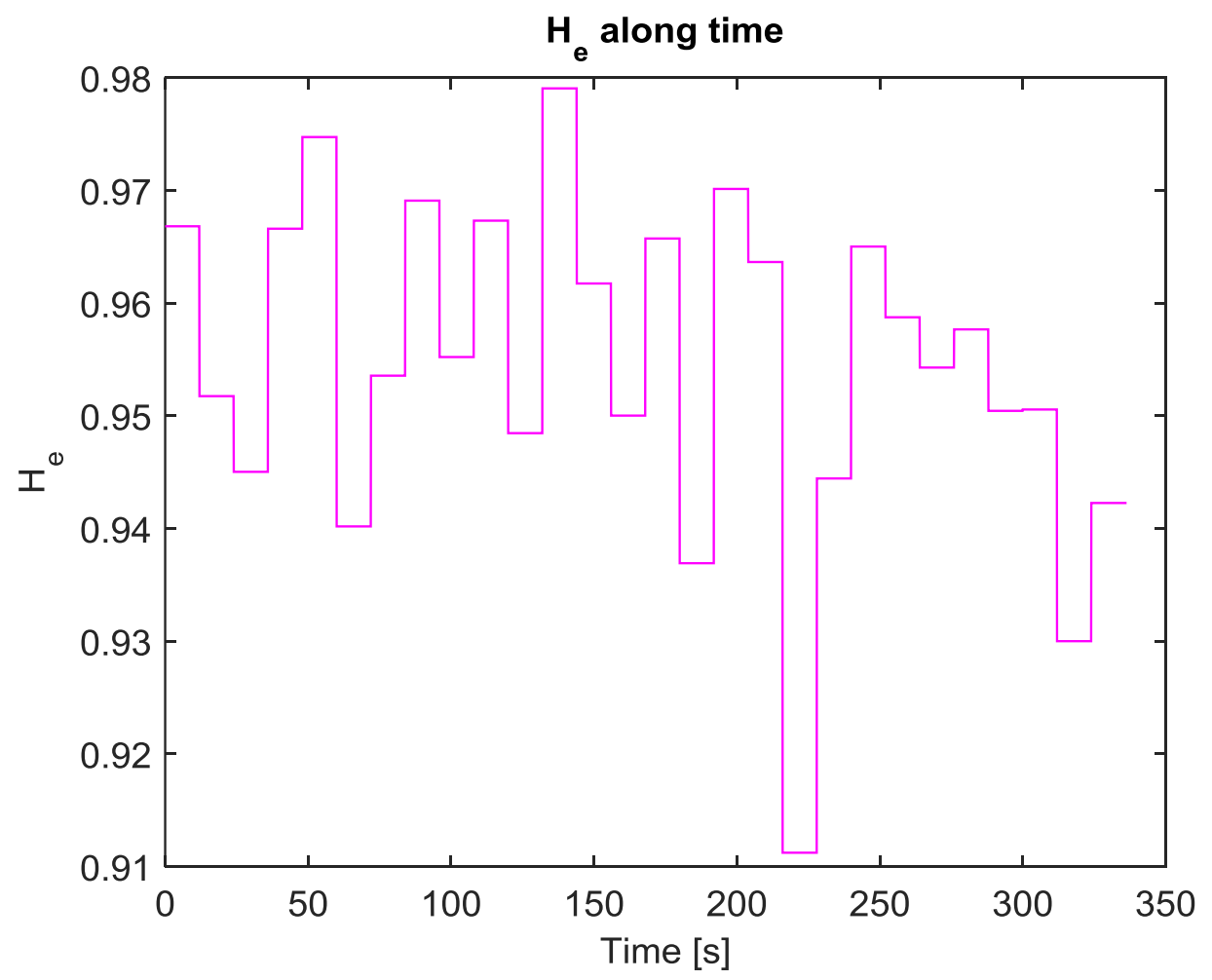

Figure 124. Estimated $\mathrm{H}_{\mathrm{e}}$ along time for the Forsmark case 4 APRM signal. 
Table 18. Mean(HFD) and std(HFD) of the Forsmark case 4 APRM signal.

\begin{tabular}{ccc}
\hline Forsmark case 4 APRM & mean(HFD) & std(HFD) \\
\hline 1 & 1.0453 & 0.0143 \\
\hline
\end{tabular}

Figure 125 shows the mean HFD estimates (each mean HFD value is represented by a red bullet) for the 23 signals of this Forsmark case. It is observed that from the 23 signals of the set, the HFD analysis of 4 LPRMs (LPRM 12, LPRM 14, LPRM 15 and LPRM 22) failed because their mean HFD estimates are above the stability threshold fixed empirically at 1.35. Then, chances are that such LPRM signals are transitioning from a stable state to an unstable one. Nevertheless, if the HFD based analysis of the aforementioned LPRMs is ignored and our focus goes to observe the mean HFD of the other 19/23 LPRMs, the HFD analysis of this case, confirms instability. Approximately, $83 \%$ of the studied LPRMs of this case confirm density wave instability. Figure 126 shows the mean $\mathrm{H}_{\mathrm{e}}$ of the studied signals, the mean $\mathrm{H}_{\mathrm{e}}$ values of the highlighted 4 LPRMs (LPRM 12, LPRM 14, LPRM 15 and LPRM 22) is below the stability threshold fixed at 0.65. Nevertheless, the mean $\mathrm{H}_{\mathrm{e}}$ value of the other studied 19 LPRMs of this case were above this stability threshold and overall, the performed stability (based mainly on HFD and the optional $\mathrm{H}_{\mathrm{e}}$ ) analysis pinpoints to instability. $82 \%$ of the studied LPRMs confirm unstable BWR behavior.

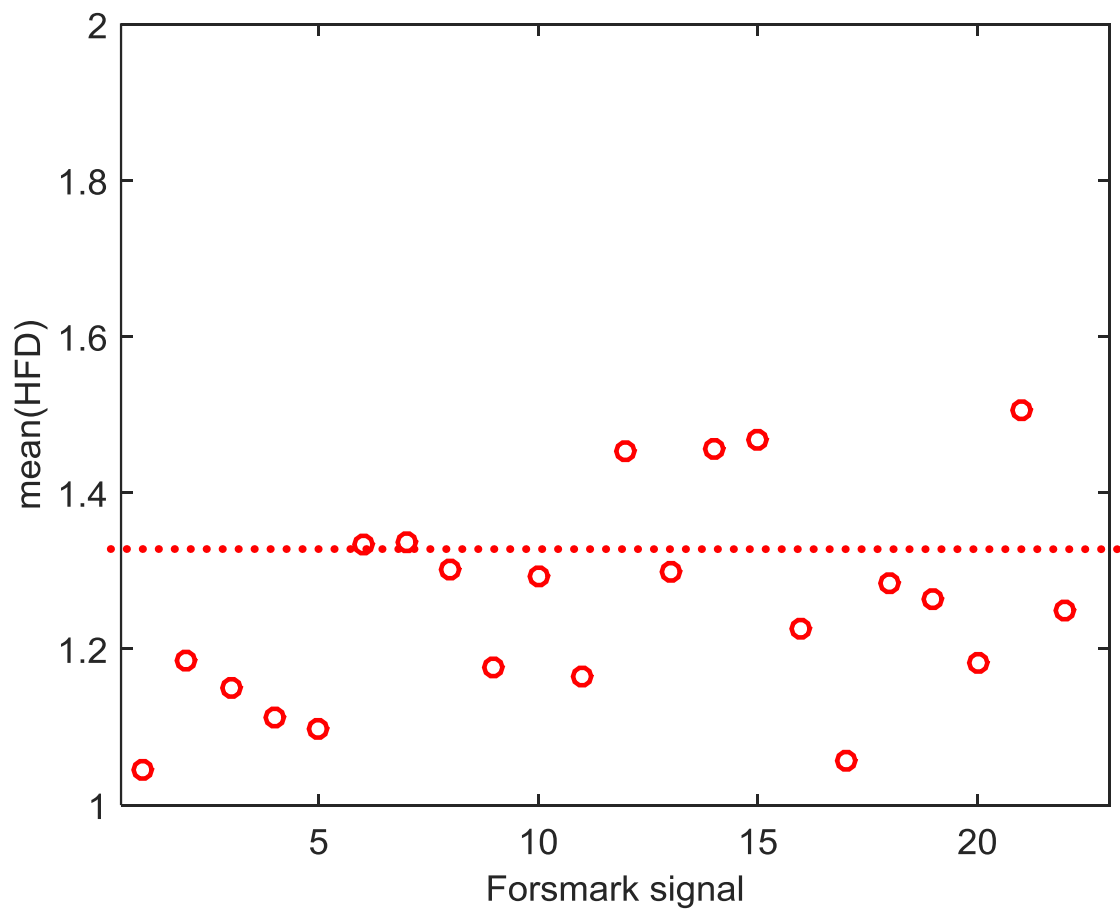

Figure 125. Mean(HFD) values for the entire Forsmark Case 4 set. 


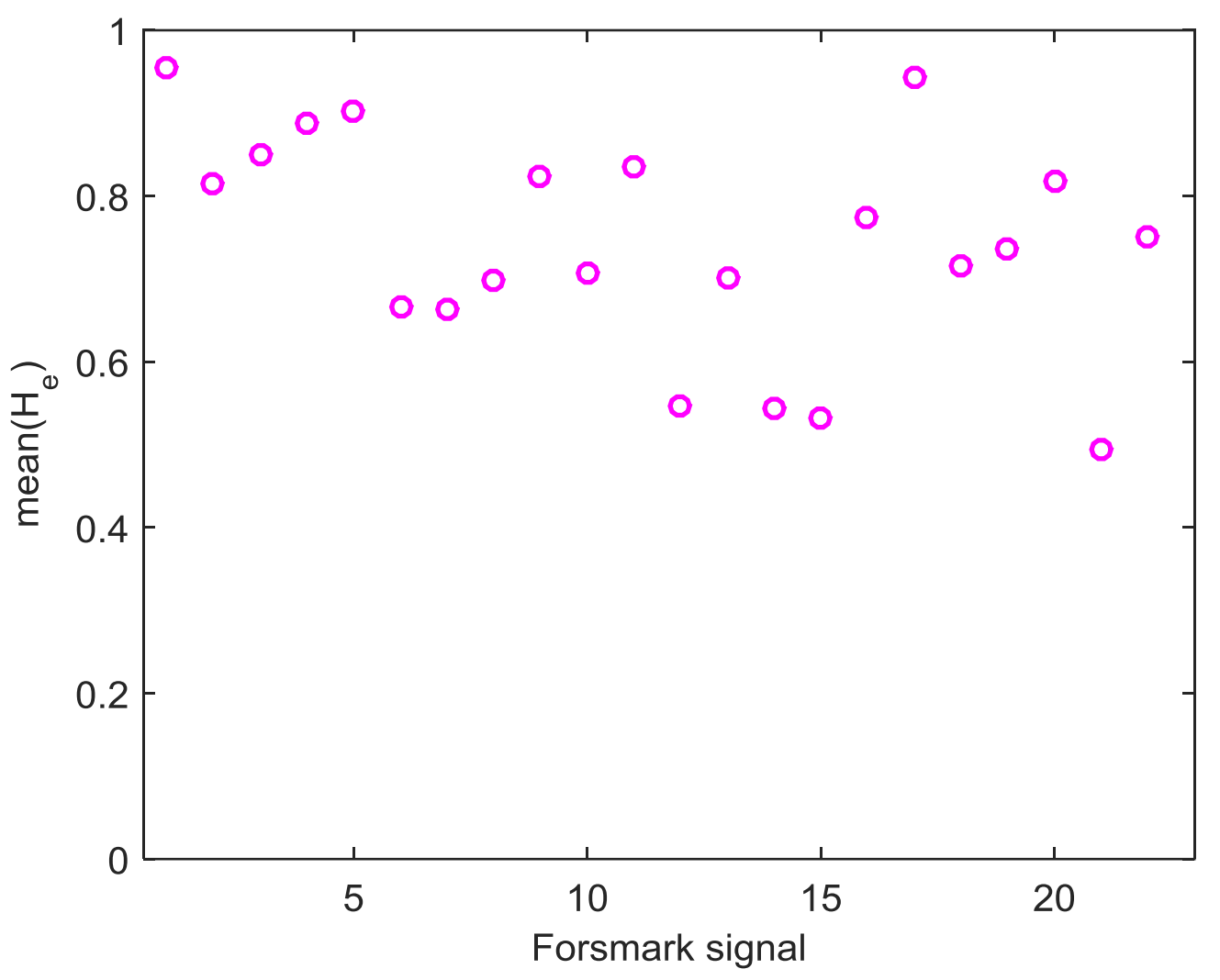

Figure 126. Mean $\left(\mathrm{H}_{\mathrm{e}}\right)$ values for the entire Forsmark Case 4 set.

\subsubsection{Case 9 cycle 14 of the Ringhals stability benchmark}

The data of this case comes from measurements of the Swedish BWR reactor Ringhals 1 (Lefvert, 1996). This case consists of a total of 72 LPRMs distributed in two floors or levels of 36 LPRMs each. As before, only the analysis of one signal (LPRM 1) is detailed in this work. Figure 127 shows the studied LPRM 1 signal, Figure 128 shows a $10 \mathrm{~s}$ studied segment of this signal. The HFD estimate of this segment is 1.053 , the HFD points to a regular sinusoidal signal, the segment is unstable. Figure 129 shows the HFD estimates along time for this LPRM 1, the HFD fluctuate around 1, pointing to instability. Figure 130 shows the estimated Hurst exponent $\mathrm{H}_{\mathrm{e}}$ for the studied signal. $\mathrm{H}_{\mathrm{e}}$ fluctuate around 1 . Thus, $\mathrm{H}_{\mathrm{e}}$ also points to BWR instability due to high regularity of the studied signal. $\mathrm{H}_{\mathrm{e}}$ oscillates around a high value above 0.9 . 


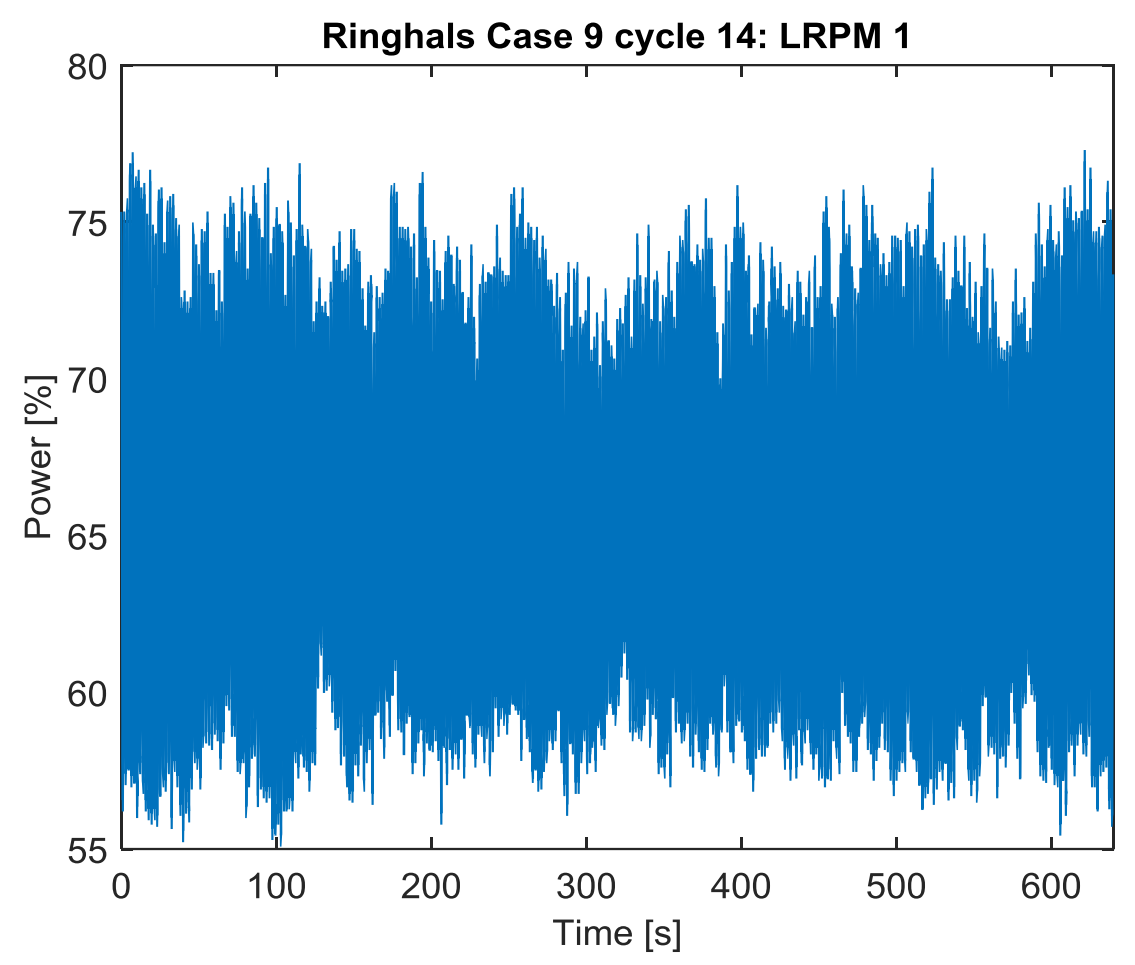

Figure 127. Ringhals case 9 cycle 14 LPRM 1.

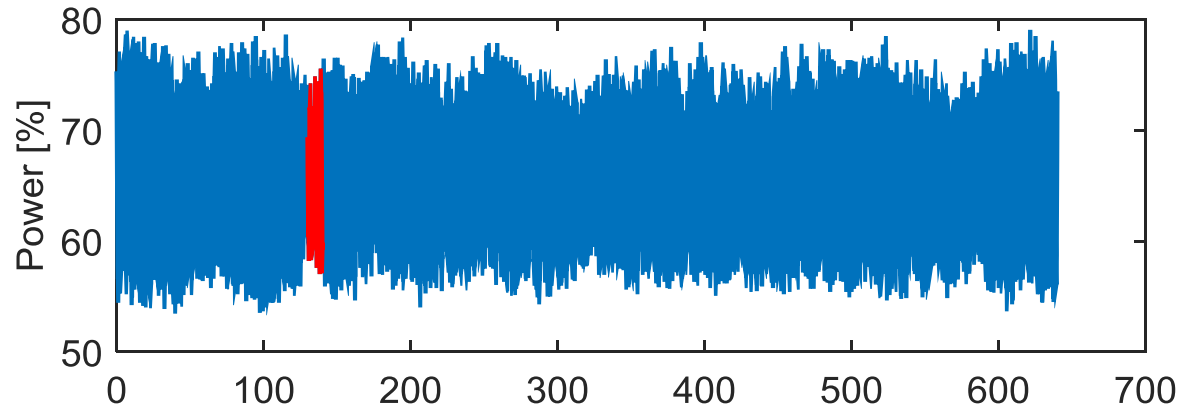

Ringhals case 9 cycle 14: LPRM 1 segment

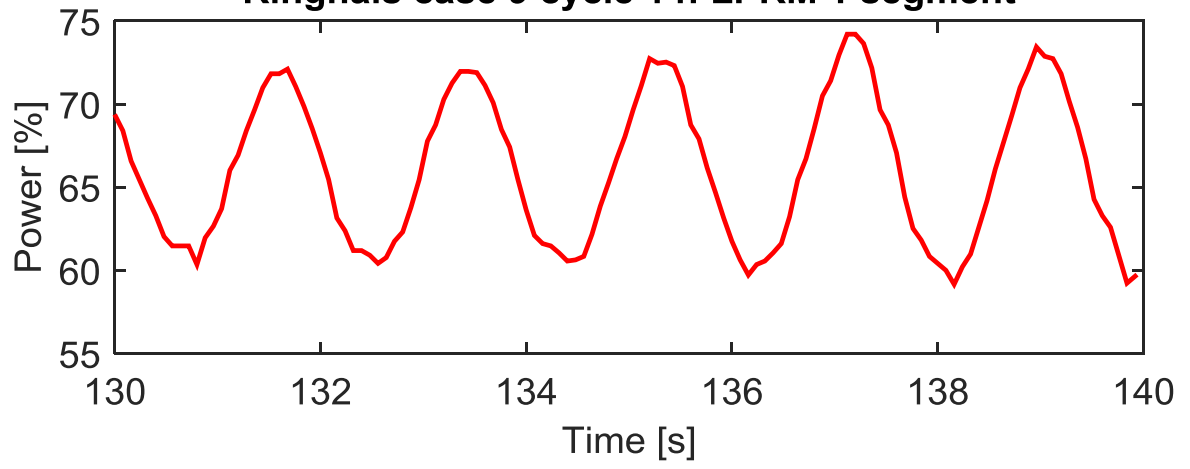

Figure 128. Studied segment of the Ringhals case 9 cycle 14 LPRM 1. 


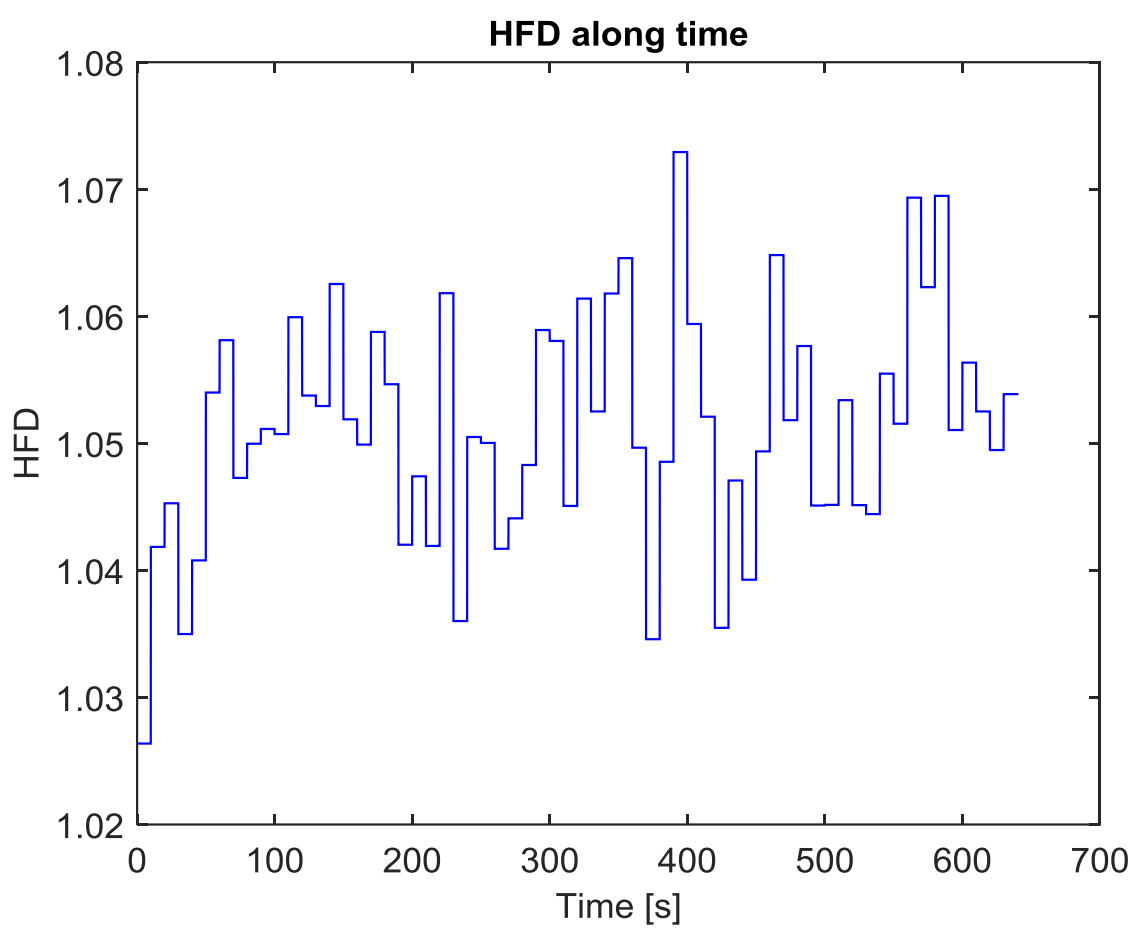

Figure 129. HFD along time for the studied Ringhals case 9 cycle 14 LPRM 1.

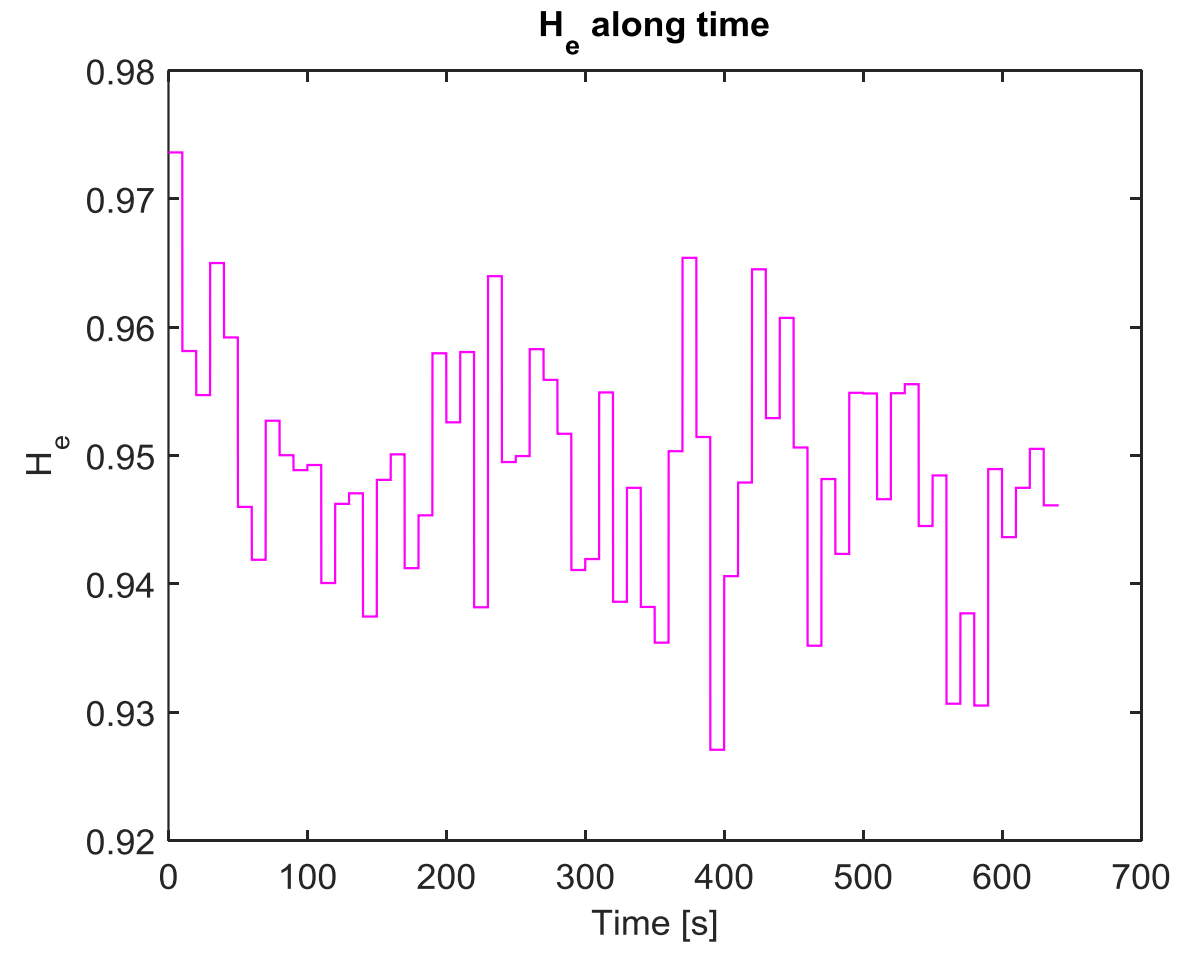

Figure 130. $\mathrm{H}_{\mathrm{e}}$ along time for the studied Ringhals case 9 cycle 14 LPRM 1. 
Table 19 shows the mean(HFD) and std(HFD) of the analyzed Ringhals LPRM 1. Figure 131 shows the computed mean(HFD) values of the 36 LPRMs of level 2 of the Ringhals reactor. It is observed, that the HFD analysis of 21/36 LPRMs pinpoint to instability, whereas the HFD analysis of 15 LPRMs point to stability. But, overall, $58 \%$ of the mean HFD values of all the studied LPRMs confirm BWR instability in floor number 2.

Table 19. Mean(HFD) and std(HFD) of the studied Ringhals LPRM 1.

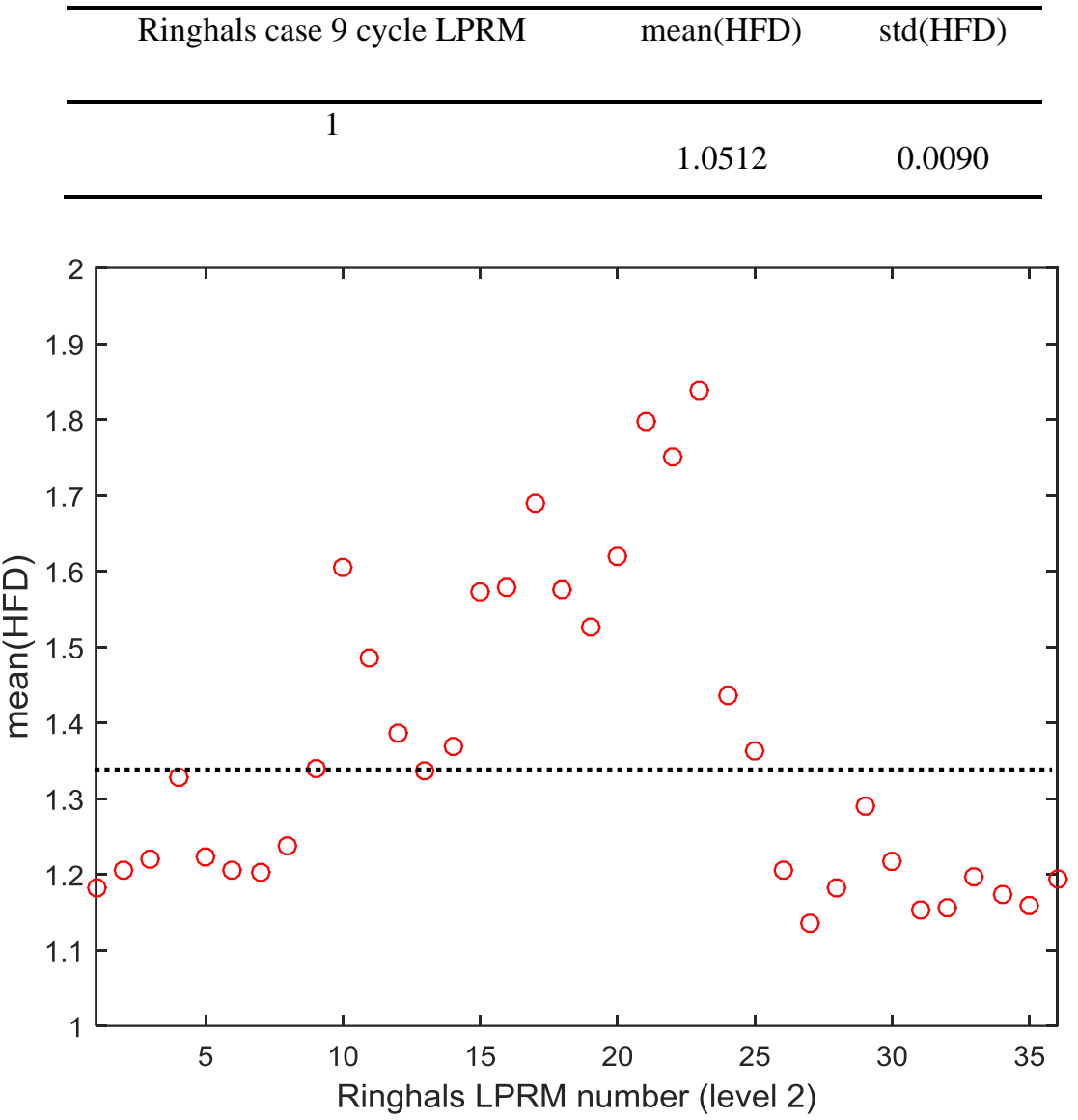

Figure 131. Mean(HFD) values for the 36 LPRMs of level 2 of the Ringhals BWR unit.

Figure 132 shows the computed mean $\left(\mathrm{H}_{\mathrm{e}}\right)$ values of the 36 LPRMs of level 2 of the Ringhals reactor. It is observed, that the $\mathrm{H}_{\mathrm{e}}$ analysis of 21/36 LPRM pinpoint to instability, whereas the $\mathrm{H}_{\mathrm{e}}$ analysis of $15 \mathrm{LPRMs}$ point to stability (the $\mathrm{H}_{\mathrm{e}}$ mean values of these 15 LPRMs is smaller than the $\mathrm{H}_{\mathrm{e}}$ threshold located at 0.65). But, overall, 58\% of the LPRMs point to instability with the optional $\mathrm{H}_{\mathrm{e}}$ indicator. 


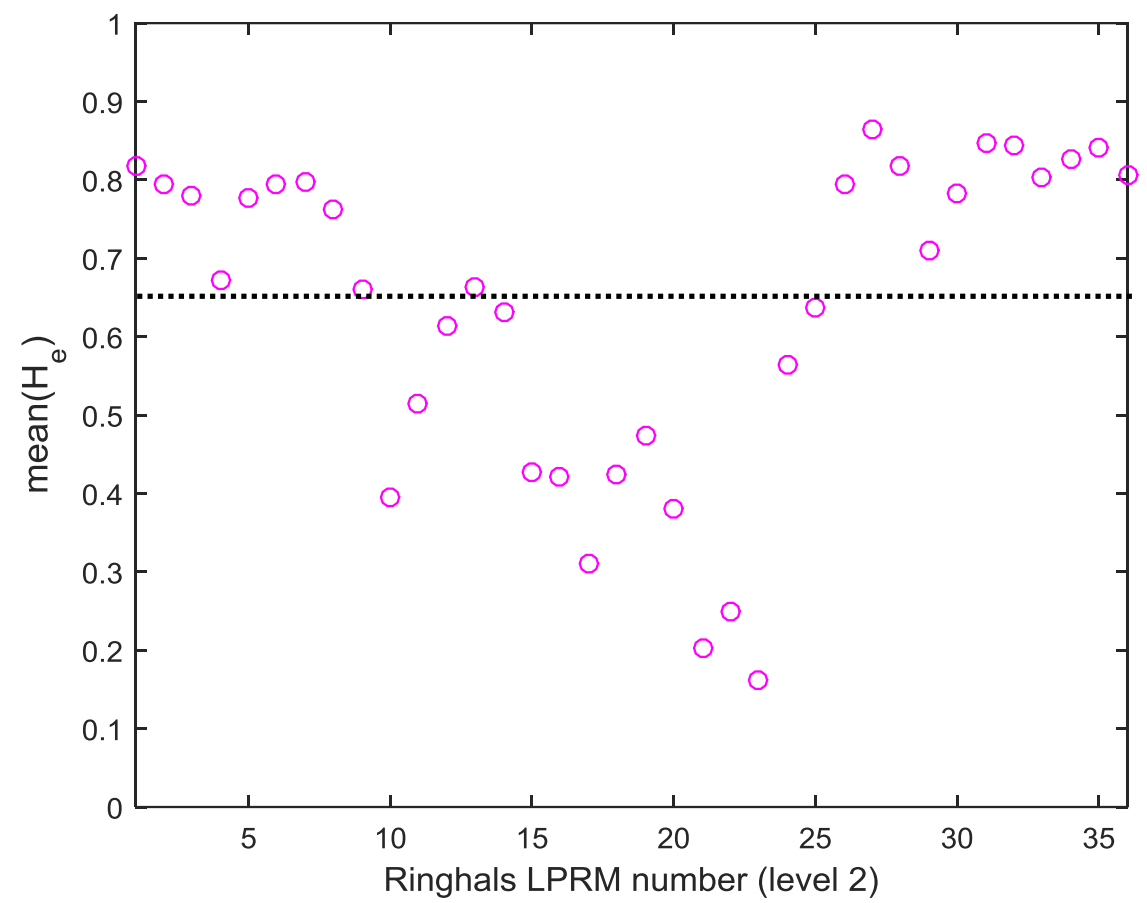

Figure 132. Mean $\left(\mathrm{H}_{\mathrm{e}}\right)$ values for the $36 \mathrm{LPRMs}$ of level 2 of the Ringhals BWR unit.

Figure 133 shows the plot of the HFD analysis of the 36 LPRMs of level 4 . In this level, the computed mean HFD of 34/36 LPRMs point to instability (the mean HFD is smaller than the HFD threshold value located at 1.35. The analysis of 2 LPRMs points to stability. However, in this case, $\approx 95 \%$ of the analyzed LPRMs point to instability. So, level 4 is mostly unstable. Finally, Figure 134 shows the computed mean $\left(\mathrm{H}_{\mathrm{e}}\right)$ values of the 36 LPRMs of level 4 and analogously, the mean $\mathrm{H}_{\mathrm{e}}$ values of 34 LPRMs indicate BWR instability (the mean $\mathrm{H}_{e}$ values of 34 LPRMs is greater than the $\mathrm{H}_{e}$ stability threshold value of 0.65). Thus, level 4 is unstable and the analysis of $95 \%$ of the LRPMs indicate in here undesirable density wave instability. 


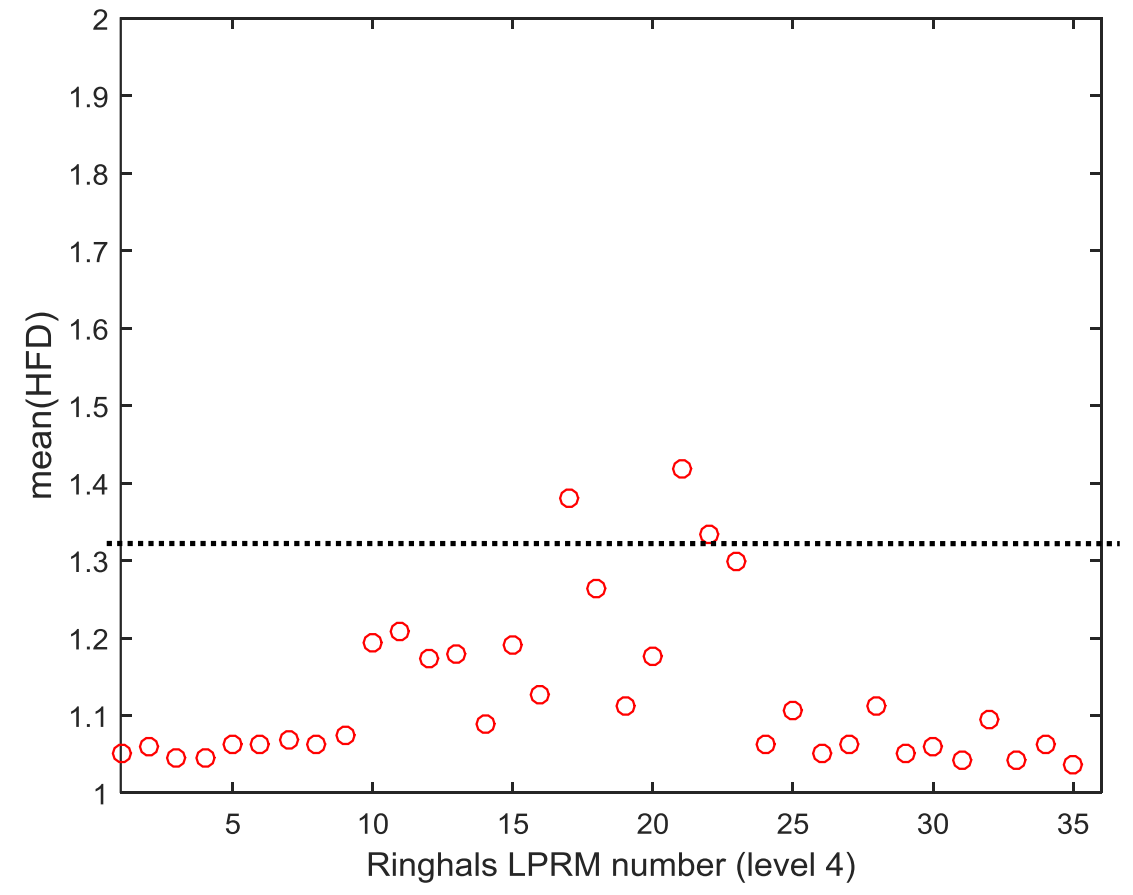

Figure 133. Mean(HFD) values for the 36 LPRMs of level 4 of the Ringhals BWR unit.

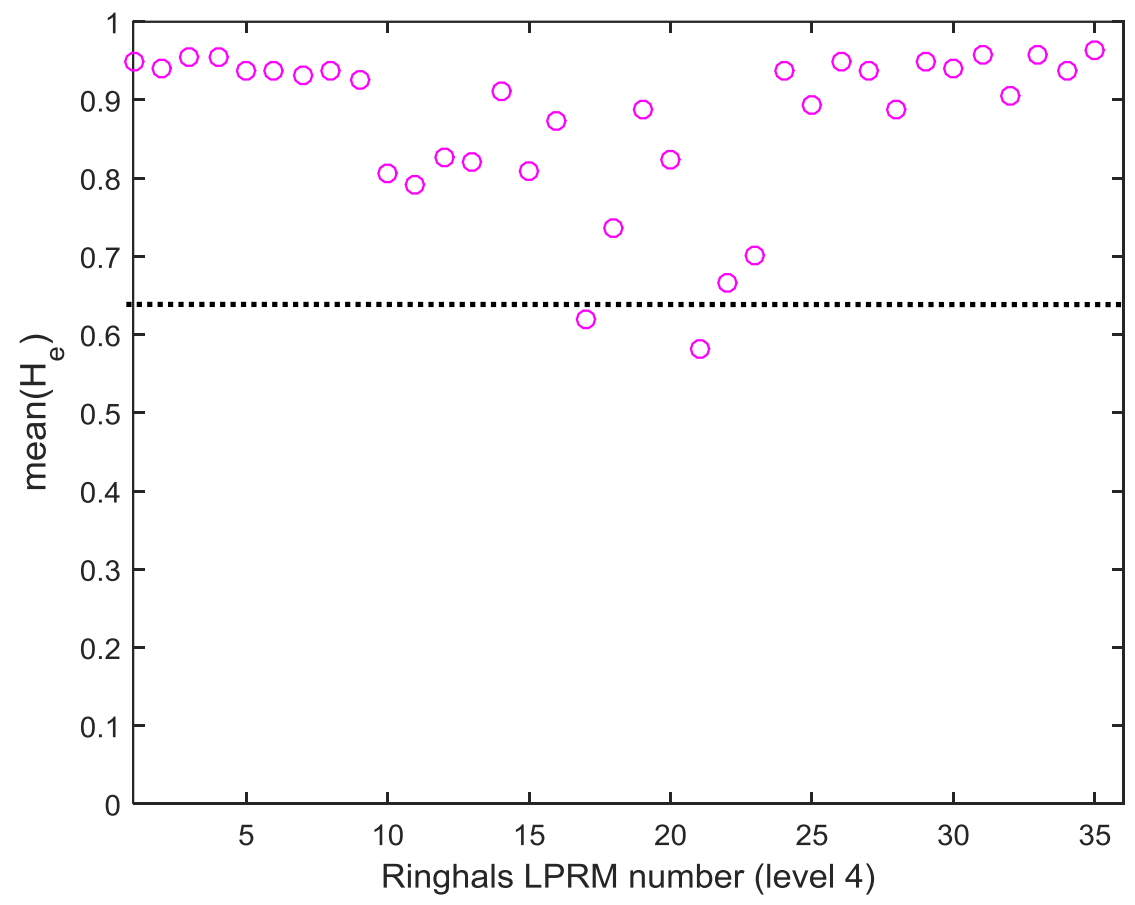

Figure 134. Mean $\left(\mathrm{H}_{\mathrm{e}}\right)$ values for the $36 \mathrm{LPRMs}$ of level 4 of the Ringhals BWR unit. 


\subsection{Chapter 7 discussions}

In this chapter, a fractal dimension estimator known as the Higuchi fractal dimension (HFD) was applied to develop a non-linear methodology for BWR stability. The HFD was tested to accommodate the complex dynamics of a BWR during instability events. The HFD measures the complexity (i.e. roughness) of BWR signals. Via the observation of signal complexity through HFD it is possible to detect and classify stable signals from unstable ones. Numerical experiments with artificial signals were performed to infer the best possible value for the HFD free parameter $\mathrm{k}_{\max }$ for our BWR signal stability analysis.

It was found that the best possible $\mathrm{k}_{\max }$ value to hit the jackpot (the best possible HFD estimate for the studied BWR signals, which is the closest estimate to the true FD value) is when $\mathrm{k}_{\max }$ is fixed to 4 . All in agreement with the intensive experiments performed with complex Fractional Gaussian noise (fGn) paths. We hope that the information revealed by our findings might slightly contribute to the growth of the scientific literature surrounding the HFD. We found that the best $\mathrm{k}_{\max }$ are located within the interval $[3,5]$ whereas higher $\mathrm{k}_{\max }$ values lead to poorer HFD estimates (Figures 103-106).

The proposed HFD analysis rolling window methodology permitted to classify stable BWR signals from unstable ones. The computed mean HFD values of stable signals, oscillated around 1.5 (a high HFD value is associated to high signal irregularity, the studied signal might be pure noise around the nominal value), such high HFD estimates point to noisy signals. The HFD values of stable signals fluctuate around 1 (such HFD values are linked to low signal complexity, the studied signal in this case is a sinusoidal one), pointing to a sinusoidal signal associated to BWR instability. $83 \%$ of the Forsmark case 4 studied LPRMs confirm BWR density wave instability through our proposed HFD stability analysis. 58\% of the studied LPRMs of level 2 of the Ringhals case 9 cycle 14 confirm instability and $95 \%$ of the studied LPRMs of level 4 of this same Ringhals case confirm instability. Thus, 76\% (55/72) of the studied 72 LPRMs of the studied Ringhals case confirm density wave instability. According to these experiments the HFD is a suitable candidate to be used as a BWR stability indicator. 


\section{Chapter 8}

\section{A non-linear stability monitor for boiling water reactors based on the Higuchi fractal dimension with decision rules}

In the past three chapters, we have studied the potentialities of three different non-linear measurements to assess the complexity of time series with the goal of detecting oscillations related to density wave instabilities in BWRs. In here we discuss the constraints of the three studied indicators. The strongest indicator among the three studied ones is chosen to build a robust and practical BWR stability monitor for DW oscillation detection with decision rules.

The studied Shannon Entropy estimator has the following limitations:

The $c_{i} \in\left\{\mathrm{c}_{i}, \ldots, \mathrm{c}_{M}\right\}$ values that constitute $c$ (the studied LPRM signal) must be independent and identically distributed random variables. This is not achieved in practice because there is always a correlation between the samples that make up a LPRM time series. Besides, there is the need to estimate the optimal $M$ number of bins to build a histogram to compute $H_{s}(c)$ (SE, Equation (22) ). Such optimal estimation of $M$ is achieved through a complex Bayesian formula given in Knuth, 2006. In other words, we have two constraints: we are not fully respecting the Shannon Entropy theoretical rules and that the selected Bayesian formula to calculate the required Histogram for SE calculation is not a simple one.

The SampEn has the following limitations:

The studied time series must be too long to provide reliable SampEn estimates. In the case of LPRM recordings, the time span of the studied time series must be of a minimum of $60 \mathrm{~s}$ of duration (For instance, if the sample frequency of the time series is $12.5 \mathrm{~Hz}$, a $60 \mathrm{~s}$ signal sample has a total of 750 data points). We can do better and we can detect the density wave oscillation with shorter time spans of data. Besides, the SampEn requires two input parameters: $m$ and $r$ (Richman and Moorman, 2000). In the case of studied BWR signals, those two parameters were fixed at their default values of $m=2$ and $r=$ std(time_series), where std is standard deviation and time_series is the studied LPRM recording.

The Higuchi Fractal dimension (HFD) has only one limitation: there is not enough information regarding the selection of the $\mathrm{k}_{\max }$ input HFD parameter. But according to the 
experiments shown in section 7.3, a reliable $\mathrm{k}_{\max }$ value is one selected in the interval [2,5]. We fixed $k_{\max }$ at 4 for our experiments with LPRMs and according to our observations $\mathrm{k}_{\max }=4$ worked incredibly well for our HFD computations of BWR signals. Besides, the time span of the BWR studied time series for HFD estimation is of $10 \mathrm{~s}$. HFD provides credible fractal dimension estimates with few data points. The HFD proved to be the strongest proposal for BWR instability appraisal (for as long as the BWR instability event is triggered by Density Waves). In the Following, a stability monitor rooted in the Higuchi Fractal Dimension (Higuchi, 1988) will be presented. The main goal of this chapter is to introduce to the BWR scientific community the potentialities of studying the roughness of local power range monitors time series through fractal analysis to detect core stability instead of relying on conventional power spectrum analysis techniques to achieve this important goal for this type of nuclear reactors, the detection of instabilities. This monitor is tested with real unstable signals that are provided by the Forsmark and Ringhals stability frameworks and with stable signals that stem from a typical BWR. The proposed monitor is capable of classifying stable states from unstable ones.

\subsection{Background of the proposal}

A stability monitor is a system that detects BWR induced unstable power oscillations. In an ideal scenario, a stability monitor prevents unstable oscillations altogether. The stability monitor uses as input data, the recordings of local power range monitors (LPRMs) to detect the instability of the core. The most common induced unstable power oscillation for BWRs is that associated with the density wave (March-Leuba and Rey, 1993). The density wave (DW) is the most common instability type that has been observed in real life BWR operation. DWs can be described as follows: given a flow disturbance, a wave of voids travels upwards through the channel inducing a pressure drop that is delayed with respect to the original disturbance. An increase in flow might induce an increase in pressure drop and a negative feedback that reduces the flow disturbance. The DW phenomena, delays such feedback, and, at some frequency, the delay is equivalent to a phase lag. So, at this frequency (which is very close to $0.5 \mathrm{~Hz}$ ), the pressure drop feedback is positive. If the gain is sufficiently large enough, the channel flow becomes unstable and oscillates at that frequency highly concentrated around $0.5 \mathrm{~Hz}$. Today, the most popular indicator to study BWR stability due to density wave oscillations is the decay ratio (DR), an indicator that is calculated from an estimate of the impulse response function of the BWR core. The DR is the only output indicator out-of-possibly all of the stability monitors proposed in the literature (Van der Hagen et al., 2000). The DR has been quite accepted and it is an easy indicator to implement in practice. Several stability monitors have been proposed in the past to detect and suppress unstable behavior in BWRs. Such as the ones given in: Yokomizo et al., 1990; Mitsutake et al., 1982 ; Covington and Noël, 2003; Grandi et al., 2011; Anegawa et al., 1996; Mowry and Nir, 2001; Tsuji et al., 2005; Prieto-Guerrero et al., 2015. All of the mentioned works provide DR estimates to appraise BWR intability, none of the mentioned proposals dared to explore non-linear indicators (to accommodate for nonlinearity and non-stationarity of data). We propose a novel BWR stability monitor based on the Higuchi fractal dimension (HFD). This technique estimates the fractal dimension of the 
BWR signals to infer whether or not they are stable. The fractal dimension is a measurement that provides an index of roughness or complexity of a studied pattern (Higuchi, 1988).

\subsection{Stability monitor based on the HFD}

The novel methodology to detect stable or unstable states in a BWR core is presented in the next steps:

Step 1. Let $\mathrm{N}$ be the number of LPRM signals. Where $\mathrm{N}$ is the number of LPRMs at a reactor level. Then, the N LPRMs considered are segmented in windows of $10 \mathrm{~s}$ of duration. It is important to mention that this methodology works exclusively with raw data, no need to preprocess the input signals.

Step 2. The HFD is applied locally to each LPRM of the ensemble. This step will provide N HFD estimates, one for each LPRM. Here the free parameter $k_{\max }$ is fixed at 4 (as we discussed at the beginning of this section).

Step 3. Once the estimation of the $\mathrm{N}$ different HFD values has been computed. The decision rule is applied, which is presented in Section 3.1 of this work.

\subsubsection{Decision rule}

The decision rule used for to the local HFD estimates (of the $\mathrm{N}$ - LPRMs) considers that if $75 \%$ of the $\mathrm{N}$ - HFD estimates are below a HFD threshold set to 1.4 (indicating instability), a HFD alarm (HFDA) is set to 1 for the $10 \mathrm{~s}$ time segment. Else HFDA is set to 0.

Now, if five consecutive time segments (of $10 \mathrm{~s}$ of duration each) are set to 1 , a General HFD alarm (GHFDA) is set to 1 (indicating unstable behavior), otherwise the GHFDA is set to 0 (indicating stable behavior).

The proposed methodology is illustrated in Figures 135 and 136. Figure 135 illustrates Steps 1 to Step 3 whereas Figure 136 shows how decision rule number one is applied. 


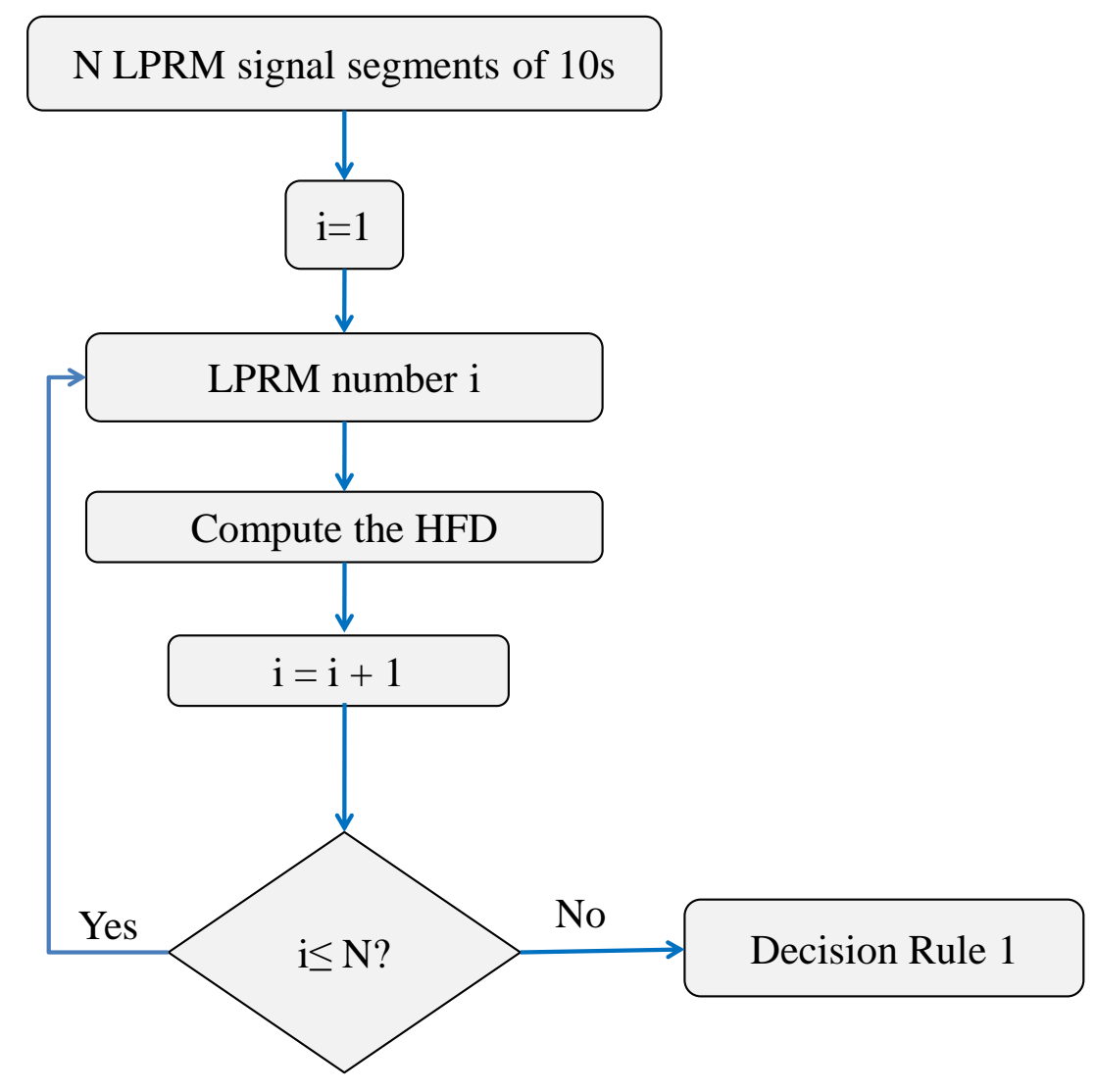

Figure 135. Flow chart to compute the HFD of raw LPRM signals. 


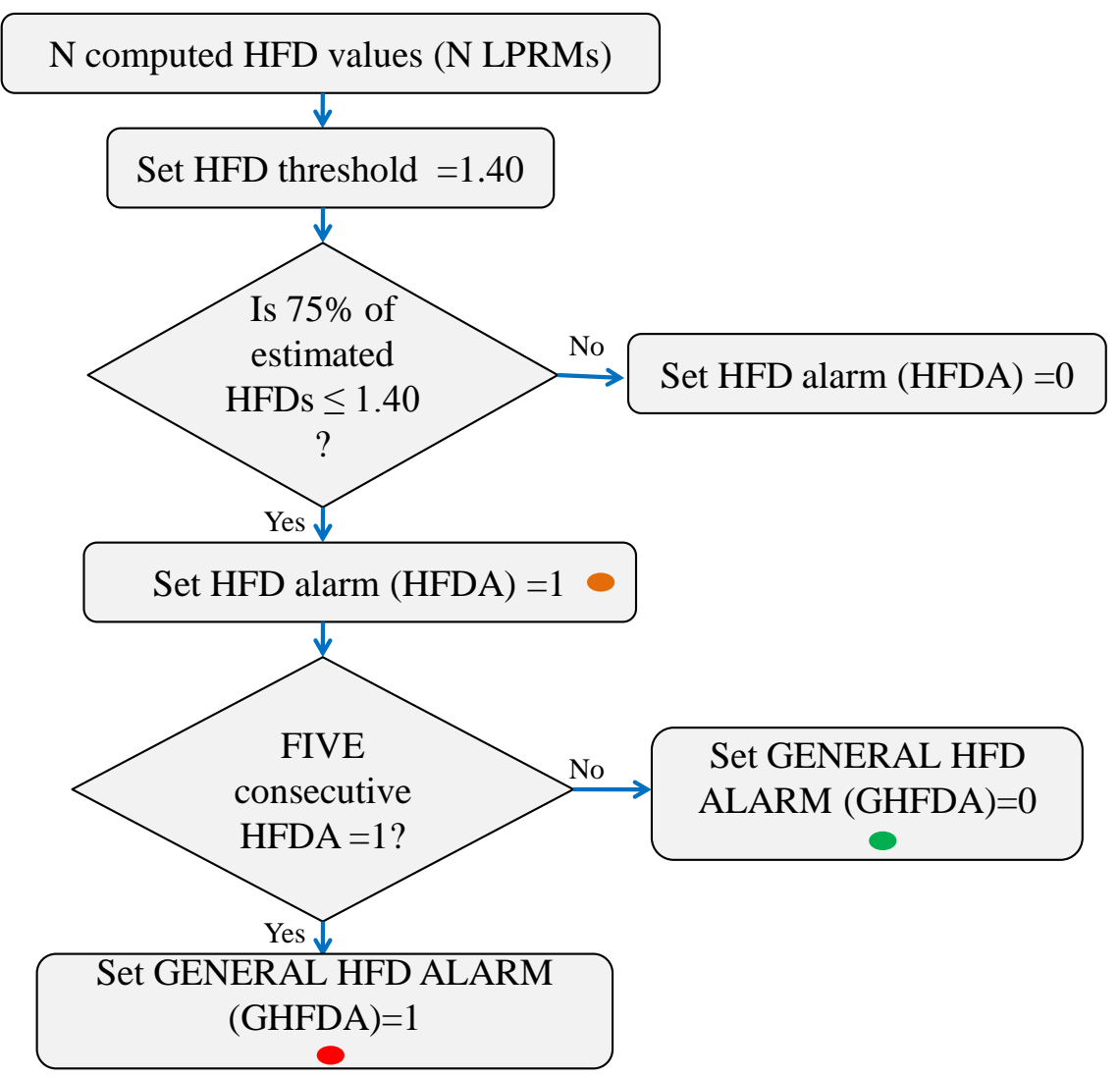

Figure 136. Flow chart of decision rule.

\subsection{Validation}

Now we validate the stability monitor given in Section 3 with the next data sets, where $f_{s}$ is the sampling frequency of each set:

1. Case 9 cycle 14 of the Ringhals stability benchmark $\left(f_{\mathrm{s}}=12.5 \mathrm{~Hz}\right)$ :

72 LPRM signals (located within 2 levels of the Ringhals reactor, each level has a total of 36 LPRMs, such levels are called 2 and 4).

2. Case 4 of the Forsmark stability benchmark $\left(f_{\mathrm{s}}=12.5 \mathrm{~Hz}\right)$ :

22 LPRM signals and 1 APRM signal (a sample of 22 LPRM signals located within two levels of the Forsmark reactor).

3. Stable signals of a Typical BWR $\left(f_{\mathrm{s}}=5 \mathrm{~Hz}\right)$

A data set of 207 steady state condition APRM signals. 


\subsubsection{Case 9 cycle 14 of the Ringhals stability benchmark}

The data used in this case comes from measurements of the Swedish BWR reactor Ringhals (Lefvert, 1996). This Ringhals framework was developed to enable code developers in member countries to test their codes to validate their predictive capability to detect BWR instabilities. Data comes from measurements in BOC 14 and 15 in the Swedish BWR reactor Ringhals 1, designed by $\mathrm{ABB}$ Atom and operated by Vattenfall $\mathrm{AB}$. The data provided by the benchmark can be used to validate and to compare models for methods used to evaluate BWR stability. The studied case consists of a total of 72 LPRMs arranged in two levels of 36 LPRMs each (such levels are called Level2 and Level4). A population of 4 LPRMs from level 2 are presented (LPRM 1 to LPRM 4). Figure 137 shows the selected 4 LPRMs and 4 segments of $10 \mathrm{~s}$ of duration of each LPRM (the $10 \mathrm{~s}$ segments are highlighted in green color). The local HFD estimates of the signals segments and the corresponding HFDA value are given in Table 20. The four HFD estimates are located below the stability threshold of 1.4. Thus, the waveforms associated to each segment are cyclic functions.
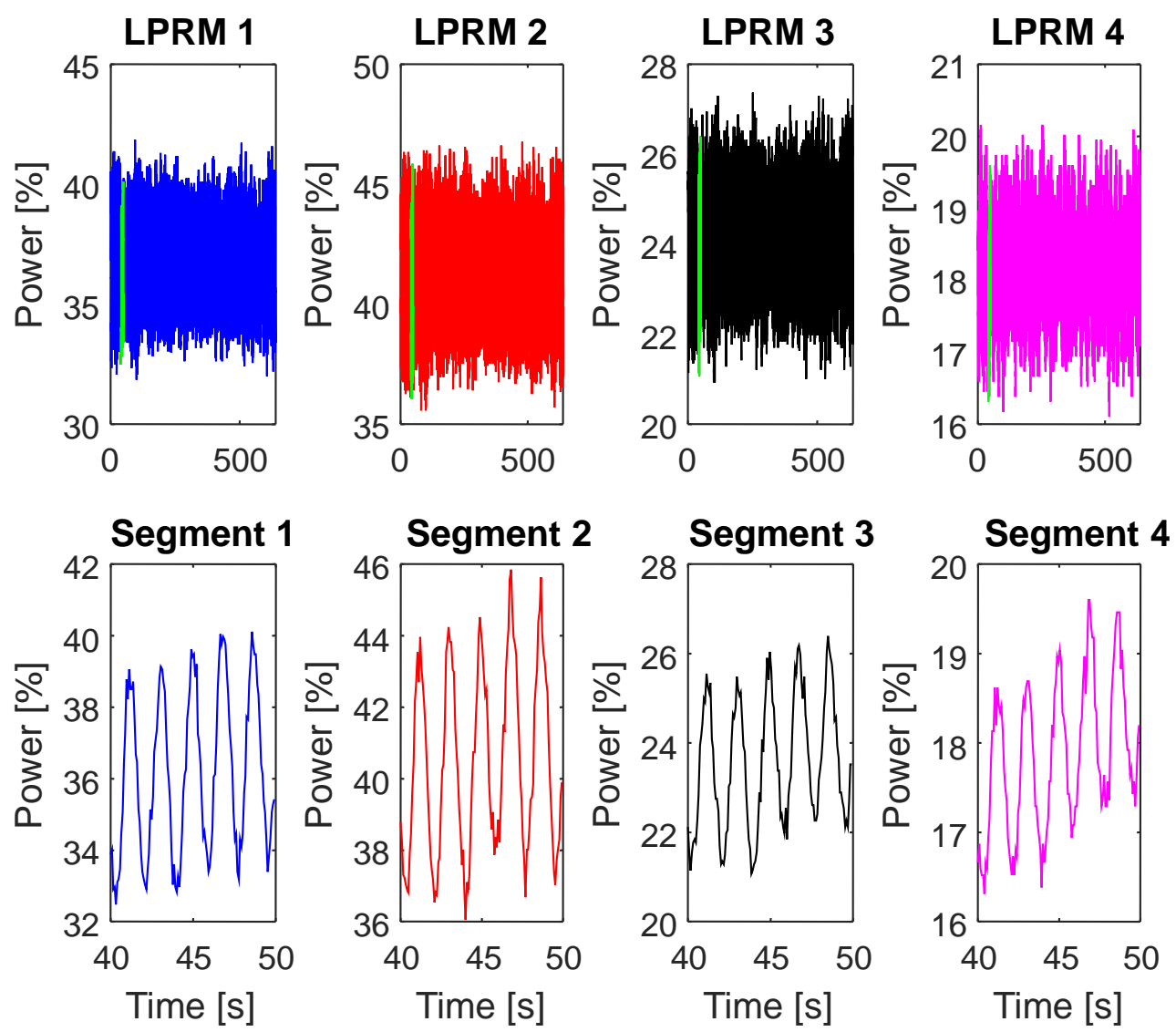

Figure 137. Ringhals NPP. Four LPRMs and their respective 10 s segment. 
Table 20. HFD estimates of the analyzed segments.

\begin{tabular}{|c|c|c|c|c|c|}
\hline \multirow{2}{*}{ HFD } & LPRM 1 & LPRM 2 & LPRM 3 & LPRM 4 & HFDA \\
\cline { 2 - 5 } & 1.1362 & 1.1660 & 1.1708 & 1.2441 & 1 \\
\hline
\end{tabular}

In Figure 137, the four signals segments look like regular functions (their respective HFD estimates will be close to 1). Table 21 shows five consecutive segments of $10 \mathrm{~s}$ and their associated HFD alarm values where the General HFD alarm turns to 1 pointing to BWR instability.

Table 21. HFD estimates of five consecutive segments.

\begin{tabular}{|c|c|c|c|c|c|}
\hline Segment \# & LPRM 1 & LPRM 2 & LPRM 3 & LPRM 4 & HFDA \\
\hline 1 & 1.1362 & 1.1660 & 1.1708 & 1.2441 & 1 \\
\hline 2 & 1.1633 & 1.1791 & 1.1851 & 1.2775 & 1 \\
\hline 3 & 1.2081 & 1.2472 & 1.2081 & 1.3426 & 1 \\
\hline 4 & 1.1700 & 1.1797 & 1.1951 & 1.2549 & 1 \\
\hline 5 & 1.2031 & 1.1587 & 1.1831 & 1.3725 & 1 \\
\hline GHFDA & \multicolumn{5}{|c|}{} \\
\hline
\end{tabular}

Table 22 shows the complete analysis of the four selected LPRMs based on the stability monitor given in Section 3, the HFDA and GHFDA were turned on (HFDA=1 and GHFDA=1) the whole time pointing to BWR instability. In this case, $75 \%$ or more of the LPRMs were unstable.

Table 22. HFD estimates of all of the segments of the four analyzed LPRMs.

\begin{tabular}{|c|c|c|c|c|c|}
\hline Segment \# & LPRM 1 & LPRM 2 & LPRM 3 & LPRM 4 & HFDA \\
\hline 1 & 1.1715 & 1.1359 & 1.1279 & 1.2855 & 1 \\
\hline 2 & 1.0836 & 1.1323 & 1.1474 & 1.2812 & 1 \\
\hline 3 & 1.1410 & 1.1297 & 1.1750 & 1.2953 & 1 \\
\hline 4 & 1.1502 & 1.1324 & 1.1738 & 1.2636 & 1 \\
\hline 5 & 1.1362 & 1.1660 & 1.1708 & 1.2441 & 1 \\
\hline GHFDA & \multicolumn{5}{|c|}{1} \\
\hline 6 & 1.1633 & 1.1791 & 1.1851 & 1.2775 & 1 \\
\hline 7 & 1.2081 & 1.2472 & 1.2081 & 1.3426 & 1 \\
\hline 8 & 1.1700 & 1.1797 & 1.1951 & 1.2549 & 1 \\
\hline 9 & 1.2031 & 1.1587 & 1.1831 & 1.3725 & 1 \\
\hline 10 & 1.1392 & 1.1255 & 1.1698 & 1.1960 & 1 \\
\hline GHFDA & \multicolumn{5}{|c|}{1} \\
\hline 11 & 1.1355 & 1.1116 & 1.1543 & 1.2545 & 1 \\
\hline 12 & 1.1333 & 1.1601 & 1.1941 & 1.2069 & 1 \\
\hline 13 & 1.2155 & 1.2711 & 1.2653 & 1.5381 & 1 \\
\hline 14 & 1.2924 & 1.2491 & 1.2692 & 1.4129 & 1 \\
\hline 15 & 1.1965 & 1.1832 & 1.1933 & 1.2555 & 1 \\
\hline GHFDA & \multicolumn{5}{|c|}{1} \\
\hline 16 & 1.1005 & 1.2214 & 1.2122 & 1.3002 & 1 \\
\hline 17 & 1.1637 & 1.2355 & 1.2724 & 1.2623 & 1 \\
\hline 18 & 1.2013 & 1.1433 & 1.2081 & 1.2335 & 1 \\
\hline
\end{tabular}




\begin{tabular}{|c|c|c|c|c|c|}
\hline 19 & 1.1493 & 1.1898 & 1.2231 & 1.3084 & 1 \\
\hline 20 & 1.1492 & 1.1586 & 1.1623 & 1.2403 & 1 \\
\hline GHFDA & \multicolumn{5}{|c|}{1} \\
\hline 21 & 1.1557 & 1.2512 & 1.2284 & 1.2644 & 1 \\
\hline 22 & 1.1538 & 1.1248 & 1.2762 & 1.2604 & 1 \\
\hline 23 & 1.2132 & 1.2555 & 1.2417 & 1.3789 & 1 \\
\hline 24 & 1.2074 & 1.1548 & 1.1933 & 1.3243 & 1 \\
\hline 25 & 1.2202 & 1.1891 & 1.2821 & 1.3043 & 1 \\
\hline GHFDA & \multicolumn{5}{|c|}{1} \\
\hline 26 & 1.1763 & 1.2015 & 1.1998 & 1.3013 & 1 \\
\hline 27 & 1.1343 & 1.1624 & 1.1786 & 1.2962 & 1 \\
\hline 28 & 1.2158 & 1.2689 & 1.2577 & 1.2983 & 1 \\
\hline 29 & 1.1700 & 1.2061 & 1.2551 & 1.2757 & 1 \\
\hline 30 & 1.2022 & 1.2330 & 1.2828 & 1.3235 & 1 \\
\hline GHFDA & \multicolumn{5}{|c|}{ 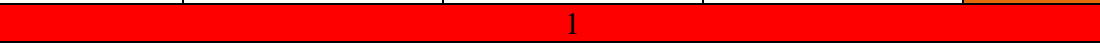 } \\
\hline 31 & 1.1981 & 1.2054 & 1.2069 & 1.3720 & 1 \\
\hline 32 & 1.3154 & 1.2630 & 1.2973 & 1.4538 & 1 \\
\hline 33 & 1.2993 & 1.3207 & 1.3179 & 1.5207 & 1 \\
\hline 34 & 1.1940 & 1.2320 & 1.2736 & 1.3240 & 1 \\
\hline 35 & 1.1942 & 1.2772 & 1.2897 & 1.4164 & 1 \\
\hline GHFDA & \multicolumn{5}{|l|}{ 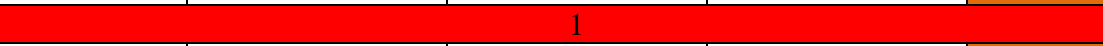 } \\
\hline 36 & 1.1687 & 1.1722 & 1.2205 & 1.3378 & 1 \\
\hline 37 & 1.1714 & 1.2109 & 1.2233 & 1.2785 & 1 \\
\hline 38 & 1.1778 & 1.1517 & 1.2887 & 1.3299 & 1 \\
\hline 39 & 1.2515 & 1.3234 & 1.2974 & 1.4857 & 1 \\
\hline 40 & 1.1656 & 1.2609 & 1.1620 & 1.4097 & 1 \\
\hline GHFDA & \multicolumn{5}{|c|}{1} \\
\hline 41 & 1.1547 & 1.2613 & 1.2253 & 1.3145 & 1 \\
\hline 42 & 1.1730 & 1.2478 & 1.2284 & 1.4333 & 1 \\
\hline 43 & 1.1755 & 1.2143 & 1.2560 & 1.3464 & 1 \\
\hline 44 & 1.1850 & 1.2395 & 1.2367 & 1.3411 & 1 \\
\hline 45 & 1.2226 & 1.2662 & 1.2114 & 1.4221 & 1 \\
\hline GHFDA & \multicolumn{5}{|c|}{1} \\
\hline 46 & 1.1489 & 1.1811 & 1.1668 & 1.2619 & 1 \\
\hline 47 & 1.1842 & 1.1622 & 1.2611 & 1.2893 & 1 \\
\hline 48 & 1.2325 & 1.2295 & 1.2568 & 1.3930 & 1 \\
\hline 49 & 1.1601 & 1.2057 & 1.1688 & 1.3131 & 1 \\
\hline 50 & 1.2150 & 1.1645 & 1.2814 & 1.3216 & 1 \\
\hline GHFDA & \multicolumn{5}{|c|}{-1} \\
\hline 51 & 1.1472 & 1.2657 & 1.2323 & 1.4018 & 1 \\
\hline 52 & 1.1135 & 1.1468 & 1.1553 & 1.3078 & 1 \\
\hline 53 & 1.1573 & 1.1828 & 1.1719 & 1.2868 & 1 \\
\hline 54 & 1.2171 & 1.1858 & 1.1697 & 1.2878 & 1 \\
\hline 55 & 1.2120 & 1.2118 & 1.2113 & 1.3546 & 1 \\
\hline GHFDA & \multicolumn{5}{|c|}{ 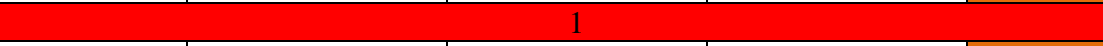 } \\
\hline 56 & 1.2439 & 1.2417 & 1.2609 & 1.3874 & 1 \\
\hline 57 & 1.2654 & 1.2653 & 1.2460 & 1.4056 & 1 \\
\hline 58 & 1.2499 & 1.2366 & 1.2836 & 1.4191 & 1 \\
\hline 59 & 1.2789 & 1.3151 & 1.3096 & 1.5519 & 1 \\
\hline 60 & 1.1113 & 1.1820 & 1.2262 & 1.2476 & 1 \\
\hline GHFDA & \multicolumn{5}{|c|}{ 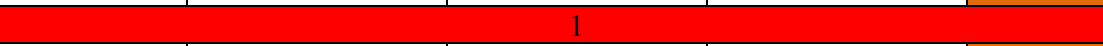 } \\
\hline 61 & 1.1440 & 1.1775 & 1.1475 & 1.2691 & 1 \\
\hline 62 & 1.1737 & 1.1810 & 1.1982 & 1.3297 & 1 \\
\hline 63 & 1.1445 & 1.1772 & 1.1354 & 1.2136 & 1 \\
\hline 64 & 1.1329 & 1.1452 & 1.1633 & 1.2829 & 1 \\
\hline
\end{tabular}


Finally, Figure 138 shows the HFD estimates along time for the four LPRMs, the red dotted line fixed at 1.4 is the HFD stability threshold point. We highlight that LPRMs 1-3 show HFD values underneath our threshold set point whereas some HFD values of LPRM 4 are above this threshold during some segments. However, to trigger the activation of the HFD alarm, the HFD estimates of $75 \%$ or more of the LPRMs must be low (HFDs $\leq 1.4$ ).

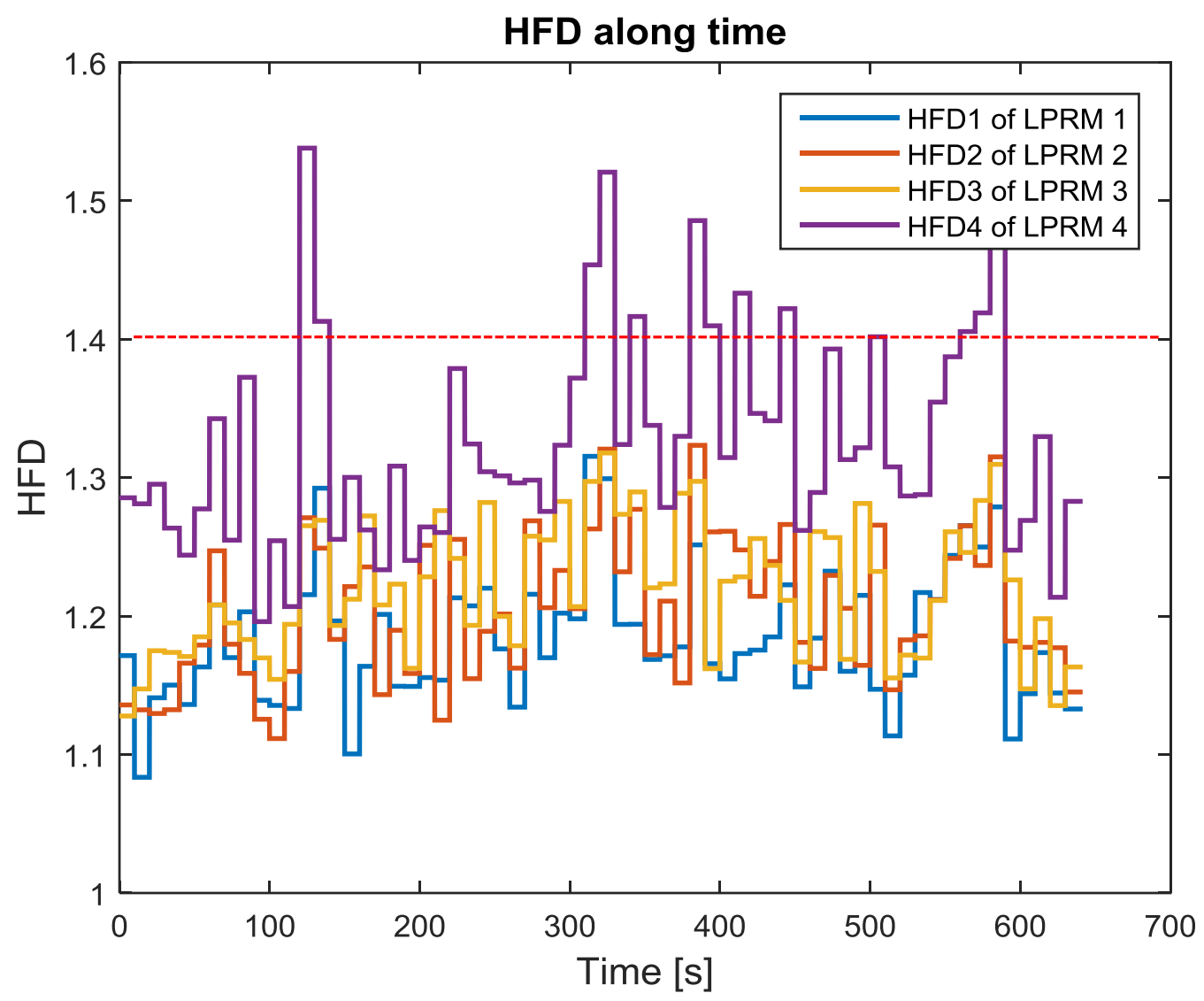

Figure 138. HFD estimates along time for the four LPRMs.

\subsubsection{Case 4 of the Forsmark stability benchmark}

Events linked to undesired power oscillations have occurred in the past in various BWR reactors. As a response, a considerable amount of research and analytical activities were developed to better understand the underlining phenomenology of the oscillations, and to define final solutions to handle such events. For the experimental study of BWR oscillations, various stability tests have been performed at the Forsmark $1 \& 2$. The Forsmark $1 \& 2$ BWR stability benchmark is the second work in a series of benchmarks based on data from Swedish BWRs. This work was coordinated by Thomas Lefvert of Vattenfall AB with the help of the team at Ringhals 1. The results were published in the report NEA/NSC/DOC96(22) in 1996 (Verdu et al., 2001). The selected event is considered by many a difficult case to be studied due to the complexity of the physical phenomenon. Again, for reasons of space, only four LPRMs will be presented. This case consists of a 
mixture between a regional and a global oscillation. Figure 139 shows the selected four LPRMs and four segments of $10 \mathrm{~s}$ of duration of each LPRM (the studied $10 \mathrm{~s}$ segments are highlighted in green color). The local HFD estimates of the studied segments and the corresponding HFDA value are given in Table 23.
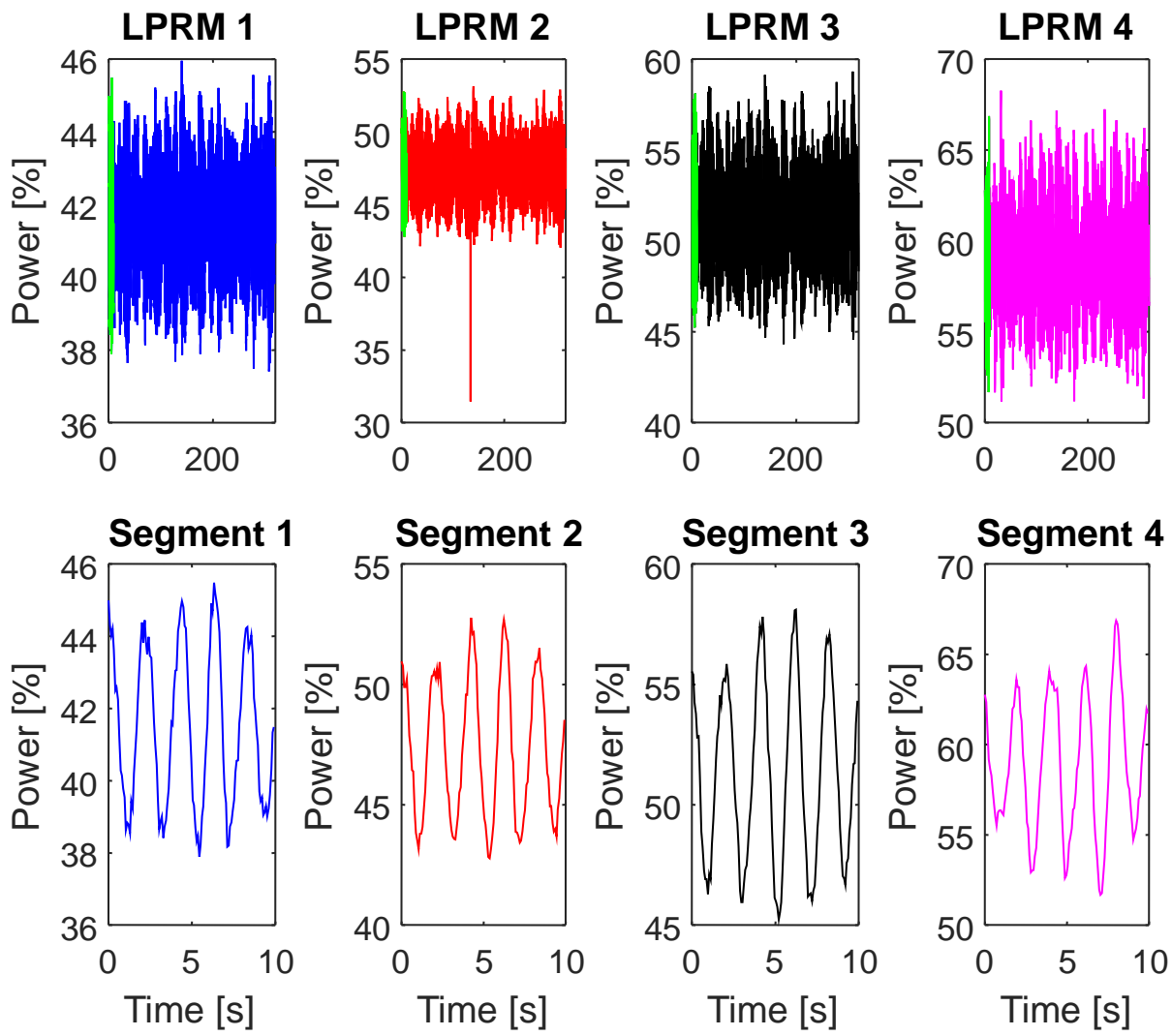

Figure 139. Forsmark stability benchmark. Four LPRMs and their respective studied $10 \mathrm{~s}$ segment.

Table 23. HFD estimates of the analyzed segments.

\begin{tabular}{|c|c|c|c|c|c|}
\hline \multirow{3}{*}{ HFD } & LPRM 1 & LPRM 2 & LPRM 3 & LPRM 4 & HFDA \\
\cline { 2 - 6 } & 1.1365 & 1.0743 & 1.0645 & 1.0540 & 1 \\
\hline
\end{tabular}

Table 24 shows five consecutive studied segments of $10 \mathrm{~s}$ and their linked HFD Alarm values. The GHFDA changes to 1 indicating the BWR instability.

Table 24. HFD estimates of five consecutive studied segments.

\begin{tabular}{|c|c|c|c|c|c|}
\hline & LPRM 1 & LPRM 2 & LPRM 3 & LPRM 4 & HFDA \\
\hline Segment ONE & 1.1365 & 1.0743 & 1.0645 & 1.0540 & 1 \\
\hline Segment TWO & 1.2166 & 1.2086 & 1.1592 & 1.1251 & 1 \\
\hline Segment THREE & 1.2010 & 1.1659 & 1.1211 & 1.1567 & 1 \\
\hline Segment FOUR & 1.1251 & 1.0747 & 1.0650 & 1.0384 & 1 \\
\hline
\end{tabular}




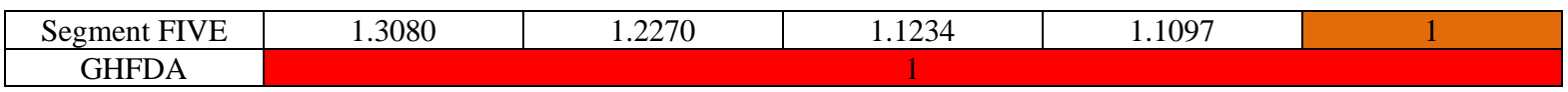

Table 25 shows the complete analysis of the four LPRMs based on the stability monitor given in Section 8.2, the HFDA and GHFDA were turned on the whole time pointing to BWR instability. In this case, $100 \%$ of the studied LPRMs were unstable.

Table 25. HFD estimates of all of the studied segments of the four analyzed LPRMs.

\begin{tabular}{|c|c|c|c|c|c|}
\hline Segment \# & LPRM 1 & LPRM 2 & LPRM 3 & LPRM 4 & HFDA \\
\hline 1 & 1.1365 & 1.0743 & 1.0645 & 1.0540 & 1 \\
\hline 2 & 1.2166 & 1.2086 & 1.1592 & 1.1251 & 1 \\
\hline 3 & 1.2010 & 1.1659 & 1.1211 & 1.1567 & 1 \\
\hline 4 & 1.1251 & 1.0747 & 1.0650 & 1.0384 & 1 \\
\hline 5 & 1.3080 & 1.2270 & 1.1234 & 1.1097 & 1 \\
\hline \multicolumn{6}{|l|}{ GHFDA } \\
\hline 6 & 1.1719 & 1.1032 & 1.0919 & 1.0838 & 1 \\
\hline 7 & 1.3084 & 1.2191 & 1.1175 & 1.0781 & 1 \\
\hline 8 & 1.1342 & 1.1722 & 1.1162 & 1.0764 & 1 \\
\hline 9 & 1.1875 & 1.1612 & 1.0947 & 1.0893 & 1 \\
\hline 10 & 1.1332 & 1.0975 & 1.0737 & 1.0825 & 1 \\
\hline \multicolumn{6}{|l|}{ GHFDA } \\
\hline 11 & 1.2809 & 1.1987 & 1.1573 & 1.0985 & 1 \\
\hline 12 & 1.1026 & 1.1019 & 1.0766 & 1.0958 & 1 \\
\hline 13 & 1.1688 & 1.1102 & 1.0790 & 1.0968 & 1 \\
\hline 14 & 1.2442 & 1.3536 & 1.1500 & 1.0931 & 1 \\
\hline 15 & 1.0730 & 1.0679 & 1.0618 & 1.0488 & 1 \\
\hline \multicolumn{6}{|l|}{ GHFDA } \\
\hline 16 & 1.2610 & 1.1630 & 1.1158 & 1.0768 & 1 \\
\hline 17 & 1.2370 & 1.2163 & 1.1852 & 1.1323 & 1 \\
\hline 18 & 1.1284 & 1.1145 & 1.0853 & 1.0494 & 1 \\
\hline 19 & 1.2525 & 1.2473 & 1.2267 & 1.2368 & 1 \\
\hline 20 & 1.1412 & 1.1181 & 1.0868 & 1.0710 & 1 \\
\hline \multicolumn{6}{|l|}{ GHFDA } \\
\hline 21 & 1.2453 & 1.1723 & 1.1199 & 1.1246 & 1 \\
\hline 22 & 1.1626 & 1.0905 & 1.0621 & 1.0677 & 1 \\
\hline 23 & 1.2708 & 1.1961 & 1.2050 & 1.0993 & 1 \\
\hline 24 & 1.1698 & 1.1449 & 1.1164 & 1.1051 & 1 \\
\hline 25 & 1.2105 & 1.1742 & 1.1854 & 1.1047 & 1 \\
\hline \multicolumn{6}{|l|}{ GHFDA } \\
\hline 26 & 1.1924 & 1.1625 & 1.0827 & 1.0919 & 1 \\
\hline 27 & 1.1095 & 1.0830 & 1.0739 & 1.0833 & 1 \\
\hline 28 & 1.1914 & 1.1411 & 1.1323 & 1.0955 & 1 \\
\hline 29 & 1.1925 & 1.1476 & 1.1343 & 1.1006 & 1 \\
\hline 30 & 1.2118 & 1.1129 & 1.0740 & 1.0692 & 1 \\
\hline \multicolumn{6}{|l|}{ GHFDA } \\
\hline 31 & 1.1316 & 1.1405 & 1.0982 & 1.0992 & 1 \\
\hline 32 & 1.1722 & 1.1219 & 1.1074 & 1.1391 & 1 \\
\hline
\end{tabular}

Figure 140 shows the HFD estimates along time for the four LPRMs. We highlight that the four LPRMs had HFD estimates underneath our threshold set point. Our entire Case 4 of the Forsmark operation is unstable. Please read Appendix A for comparisons with a DR estimation method. 


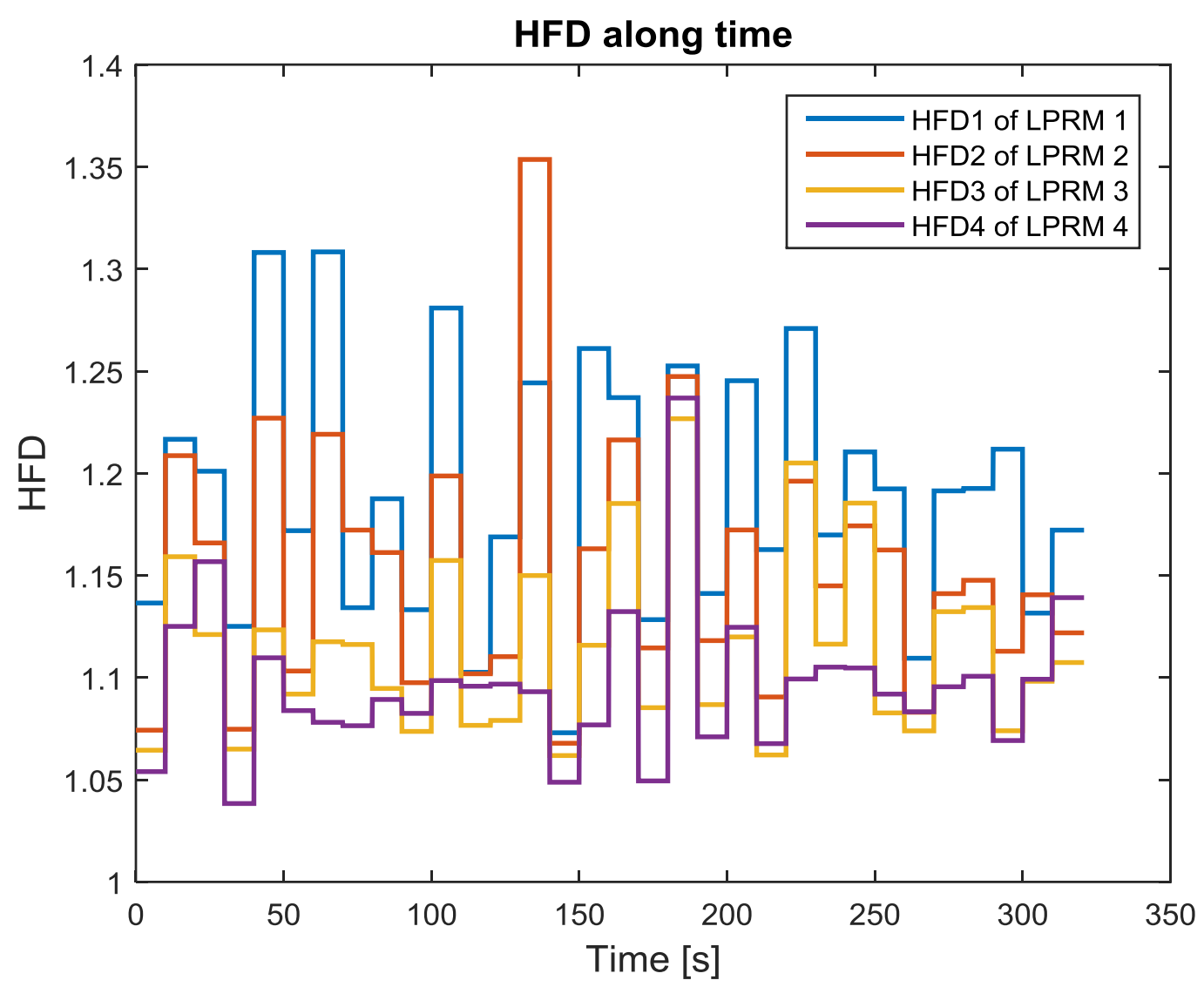

Figure 140. HFD estimates along time for the four LPRMs.

\subsubsection{Stable signals of the laguna verde reactor}

The aim of this test is to validate the proposed stability methodology with stable signals that stem from a typical BWR. The studied signals were recorded by APRMs. The four studied APRM signals and their four studied segments of $10 \mathrm{~s}$ are shown in Figure 141. The studied signals look visually like recordings of broad band non-coherent noise (BBNCN); they all look like rough paths and a priori, their HFD estimates will be high. The local HFD estimates of the studied segments and the corresponding HFDA value are given in Table 26. The HFDA value is at 0 , a sign of stable behavior. 

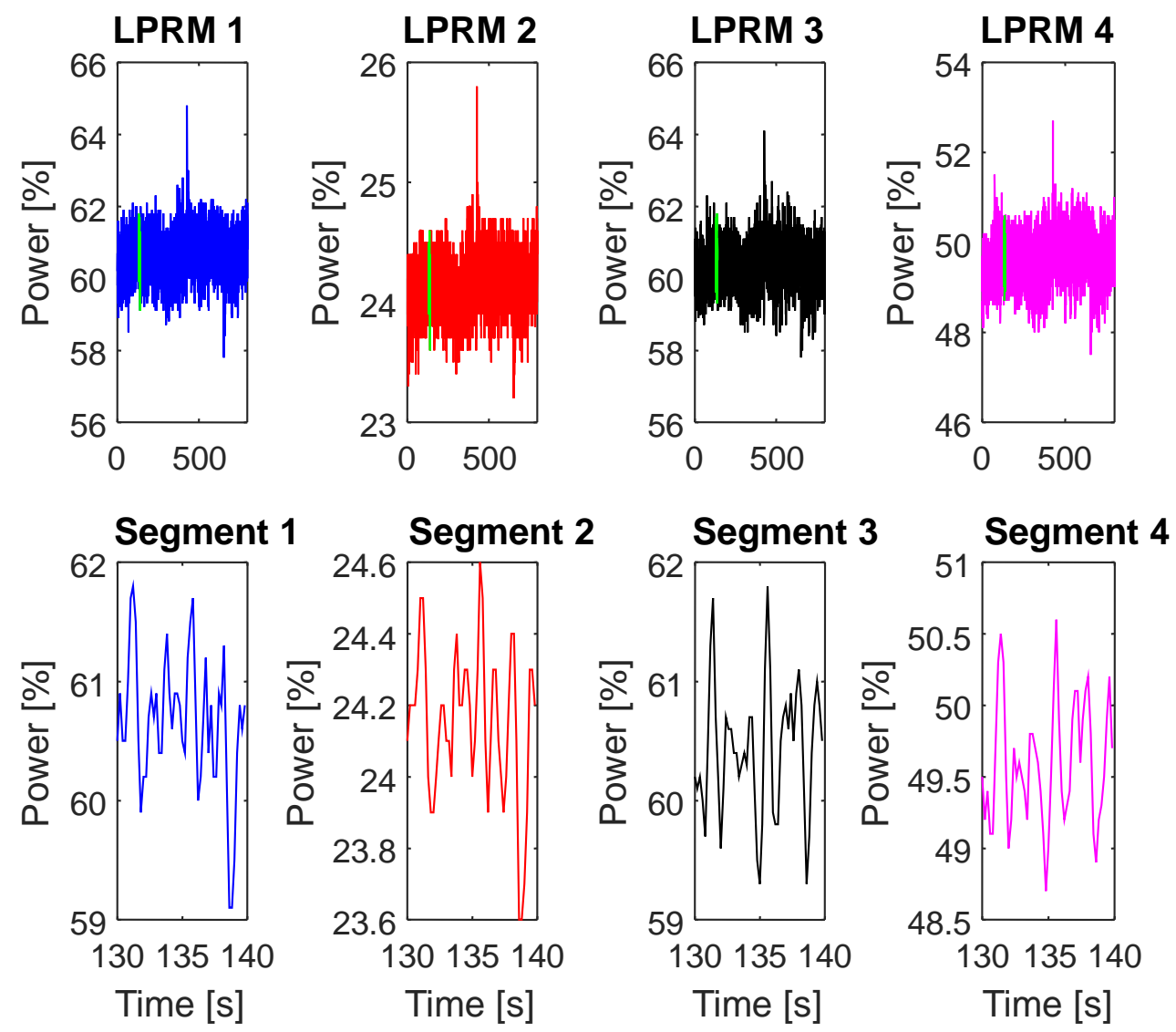

Figure 141. Four APRMs from a typical BWR and their respective studied $10 \mathrm{~s}$ segment.

Table 26. HFD estimates of the analyzed segments.

\begin{tabular}{|c|c|c|c|c|c|}
\hline \multirow{3}{*}{ HFD } & APRM 1 & APRM 2 & APRM 3 & APRM 4 & HFDA \\
\cline { 2 - 6 } & 1.5060 & 1.4398 & 1.5447 & 1.5393 & 0 \\
\hline
\end{tabular}

Table 27 shows the complete analysis of the four APRMs based on the stability monitor given in Section 8.2, the HFDA and GHFDA were turned off the whole time pointing to BWR stability. In this case, $100 \%$ of the studied APRMs were stable.

Table 27. HFD estimates of all of the studied segments of the four analyzed APRMs.

\begin{tabular}{|c|c|c|c|c|c|}
\hline Segment \# & APRM 1 & APRM 2 & APRM 3 & APRM 4 & HFDA \\
\hline 1 & 1.5060 & 1.4398 & 1.5447 & 1.5393 & 0 \\
\hline 2 & 1.5337 & 1.4424 & 1.5192 & 1.5764 & 0 \\
\hline 3 & 1.6234 & 1.4860 & 1.6180 & 1.4958 & 0 \\
\hline
\end{tabular}




\begin{tabular}{|c|c|c|c|c|c|}
\hline 4 & 1.4010 & 1.2901 & 1.4807 & 1.4805 & 0 \\
\hline 5 & 1.6027 & 1.5017 & 1.5979 & 1.4301 & 0 \\
\hline GHFDA & \multicolumn{5}{|c|}{$\overline{0}$} \\
\hline 6 & 1.5297 & 1.3768 & 1.5833 & 1.4019 & 0 \\
\hline 7 & 1.5292 & 1.5310 & 1.6392 & 1.5335 & 0 \\
\hline 8 & 1.4502 & 1.3856 & 1.4191 & 1.3278 & 0 \\
\hline 9 & 1.4540 & 1.4842 & 1.5056 & 1.3845 & 0 \\
\hline 10 & 1.5452 & 1.4811 & 1.5906 & 1.5064 & 0 \\
\hline GHFDA & \multicolumn{5}{|c|}{0} \\
\hline 11 & 1.6033 & 1.5135 & 1.6429 & 1.4565 & 0 \\
\hline 12 & 1.4877 & 1.4354 & 1.5608 & 1.4878 & 0 \\
\hline 13 & 1.7060 & 1.5111 & 1.7088 & 1.6771 & 0 \\
\hline 14 & 1.5243 & 1.4339 & 1.4879 & 1.4703 & 0 \\
\hline 15 & 1.5503 & 1.4741 & 1.6079 & 1.4883 & 0 \\
\hline GHFDA & \multicolumn{5}{|c|}{0} \\
\hline 16 & 1.6004 & 1.5173 & 1.5484 & 1.6062 & 0 \\
\hline 17 & 1.5343 & 1.5347 & 1.6752 & 1.5858 & 0 \\
\hline 18 & 1.6067 & 1.4924 & 1.5728 & 1.5415 & 0 \\
\hline 19 & 1.5985 & 1.4468 & 1.4977 & 1.4121 & 0 \\
\hline 20 & 1.5900 & 1.5324 & 1.6866 & 1.6131 & 0 \\
\hline GHFDA & \multicolumn{5}{|c|}{0} \\
\hline 21 & 1.5151 & 1.4090 & 1.5589 & 1.4596 & 0 \\
\hline 22 & 1.5027 & 1.4090 & 1.6129 & 1.4047 & 0 \\
\hline 23 & 1.5099 & 1.3754 & 1.4357 & 1.5631 & 0 \\
\hline 24 & 1.6092 & 1.5150 & 1.6682 & 1.5721 & 0 \\
\hline 25 & 1.4448 & 1.3402 & 1.6071 & 1.4979 & 0 \\
\hline GHFDA & \multicolumn{5}{|c|}{0} \\
\hline 26 & 1.5057 & 1.4228 & 1.6052 & 1.4551 & 0 \\
\hline 27 & 1.6336 & 1.6092 & 1.5335 & 1.5734 & 0 \\
\hline 28 & 1.7663 & 1.6146 & 1.9187 & 1.5602 & 0 \\
\hline 29 & 1.5608 & 1.4788 & 1.5047 & 1.5032 & 0 \\
\hline 30 & 1.4876 & 1.4237 & 1.4983 & 1.4876 & 0 \\
\hline GHFDA & \multicolumn{5}{|c|}{0} \\
\hline 31 & 1.5108 & 1.5183 & 1.6409 & 1.5730 & 0 \\
\hline 32 & 1.6431 & 1.4753 & 1.5044 & 1.5964 & 0 \\
\hline
\end{tabular}




\begin{tabular}{|c|c|c|c|c|c|}
\hline 33 & 1.4641 & 1.4076 & 1.5220 & 1.5212 & 0 \\
\hline 34 & 1.5550 & 1.4613 & 1.4328 & 1.5390 & 0 \\
\hline 35 & 1.5881 & 1.4789 & 1.6434 & 1.5577 & 0 \\
\hline GHFDA & \multicolumn{5}{|c|}{0} \\
\hline 36 & 1.6885 & 1.5792 & 1.7173 & 1.6050 & 0 \\
\hline 37 & 1.3912 & 1.3788 & 1.4921 & 1.5527 & 0 \\
\hline 38 & 1.5514 & 1.5767 & 1.6429 & 1.5689 & 0 \\
\hline 39 & 1.4517 & 1.3669 & 1.4961 & 1.4156 & 0 \\
\hline 40 & 1.4286 & 1.3513 & 1.5708 & 1.3796 & 0 \\
\hline GHFDA & \multicolumn{5}{|c|}{0} \\
\hline 41 & 1.6006 & 1.4560 & 1.6350 & 1.5591 & 0 \\
\hline 42 & 1.5692 & 1.5797 & 1.6029 & 1.5107 & 0 \\
\hline 43 & 1.4114 & 1.3609 & 1.4507 & 1.4312 & 0 \\
\hline 44 & 1.4853 & 1.4262 & 1.5736 & 1.5815 & 0 \\
\hline 45 & 1.5178 & 1.5121 & 1.5873 & 1.5252 & 0 \\
\hline GHFDA & \multicolumn{5}{|c|}{0} \\
\hline 46 & 1.5305 & 1.4133 & 1.5197 & 1.5525 & 0 \\
\hline 47 & 1.6136 & 1.5147 & 1.5006 & 1.5221 & 0 \\
\hline 48 & 1.4276 & 1.3833 & 1.4184 & 1.4045 & 0 \\
\hline 49 & 1.5073 & 1.4860 & 1.5936 & 1.5814 & 0 \\
\hline 50 & 1.5631 & 1.5333 & 1.5639 & 1.5064 & 0 \\
\hline GHFDA & \multicolumn{5}{|c|}{0} \\
\hline 51 & 1.3407 & 1.3774 & 1.4493 & 1.4702 & 0 \\
\hline 52 & 1.4421 & 1.4681 & 1.3620 & 1.3856 & 0 \\
\hline 53 & 1.6133 & 1.5675 & 1.6049 & 1.6131 & 0 \\
\hline 54 & 1.4217 & 1.4101 & 1.3223 & 1.3697 & 0 \\
\hline 55 & 1.3888 & 1.3036 & 1.4256 & 1.4945 & 0 \\
\hline GHFDA & \multicolumn{5}{|c|}{0} \\
\hline 56 & 1.5569 & 1.5362 & 1.5056 & 1.5235 & 0 \\
\hline 57 & 1.6221 & 1.6014 & 1.6452 & 1.6532 & 0 \\
\hline 58 & 1.5239 & 1.4220 & 1.4483 & 1.4742 & 0 \\
\hline 59 & 1.6034 & 1.5416 & 1.5653 & 1.5315 & 0 \\
\hline 60 & 1.3482 & 1.3412 & 1.5300 & 1.5214 & 0 \\
\hline GHFDA & \multicolumn{5}{|c|}{0} \\
\hline 61 & 1.6692 & 1.6394 & 1.6224 & 1.6186 & 0 \\
\hline
\end{tabular}




\begin{tabular}{|c|c|c|c|c|c|}
\hline 62 & 1.4870 & 1.4183 & 1.4826 & 1.6139 & 0 \\
\hline 63 & 1.6570 & 1.6058 & 1.5723 & 1.6162 & 0 \\
\hline 64 & 1.5128 & 1.4705 & 1.5924 & 1.6170 & 0 \\
\hline 65 & 1.5262 & 1.3678 & 1.5376 & 1.5889 & 0 \\
\hline GHFA & \multicolumn{5}{|c|}{0} \\
\hline 66 & 1.4433 & 1.3892 & 1.5151 & 1.5136 & 0 \\
\hline 67 & 1.5594 & 1.5080 & 1.6362 & 1.5458 & 0 \\
\hline 68 & 1.5212 & 1.5257 & 1.6052 & 1.4908 & 0 \\
\hline 69 & 1.5666 & 1.4720 & 1.6800 & 1.5631 & 0 \\
\hline 70 & 1.6192 & 1.7527 & 1.8109 & 1.6262 & 0 \\
\hline GHFDA & \multicolumn{5}{|c|}{0} \\
\hline 71 & 1.4290 & 1.3599 & 1.5220 & 1.5772 & 0 \\
\hline 72 & 1.5163 & 1.4780 & 1.6114 & 1.5324 & 0 \\
\hline 73 & 1.6266 & 1.5685 & 1.5996 & 1.4830 & 0 \\
\hline 74 & 1.5840 & 1.6164 & 1.7286 & 1.6408 & 0 \\
\hline 75 & 1.5472 & 1.4402 & 1.5458 & 1.4769 & 0 \\
\hline GHFDA & \multicolumn{5}{|c|}{0} \\
\hline 76 & 1.8917 & 1.7352 & 1.6804 & 1.5876 & 0 \\
\hline 77 & 1.5160 & 1.4969 & 1.6372 & 1.6150 & 0 \\
\hline 78 & 1.6769 & 1.6097 & 1.7058 & 1.5848 & 0 \\
\hline 79 & 1.6069 & 1.4938 & 1.5761 & 1.5933 & 0 \\
\hline 80 & 1.4640 & 1.4285 & 1.4791 & 1.4059 & 0 \\
\hline
\end{tabular}

Finally, Figure 142 shows the HFD estimates along time for the four studied APRMs. It is vital to highlight that the studied APRMs had HFD estimates most of the time higher than the threshold set point (fixed at 1.4). The 4 studied APRMs show stable behavior. The BWR is operating within the stable region. 


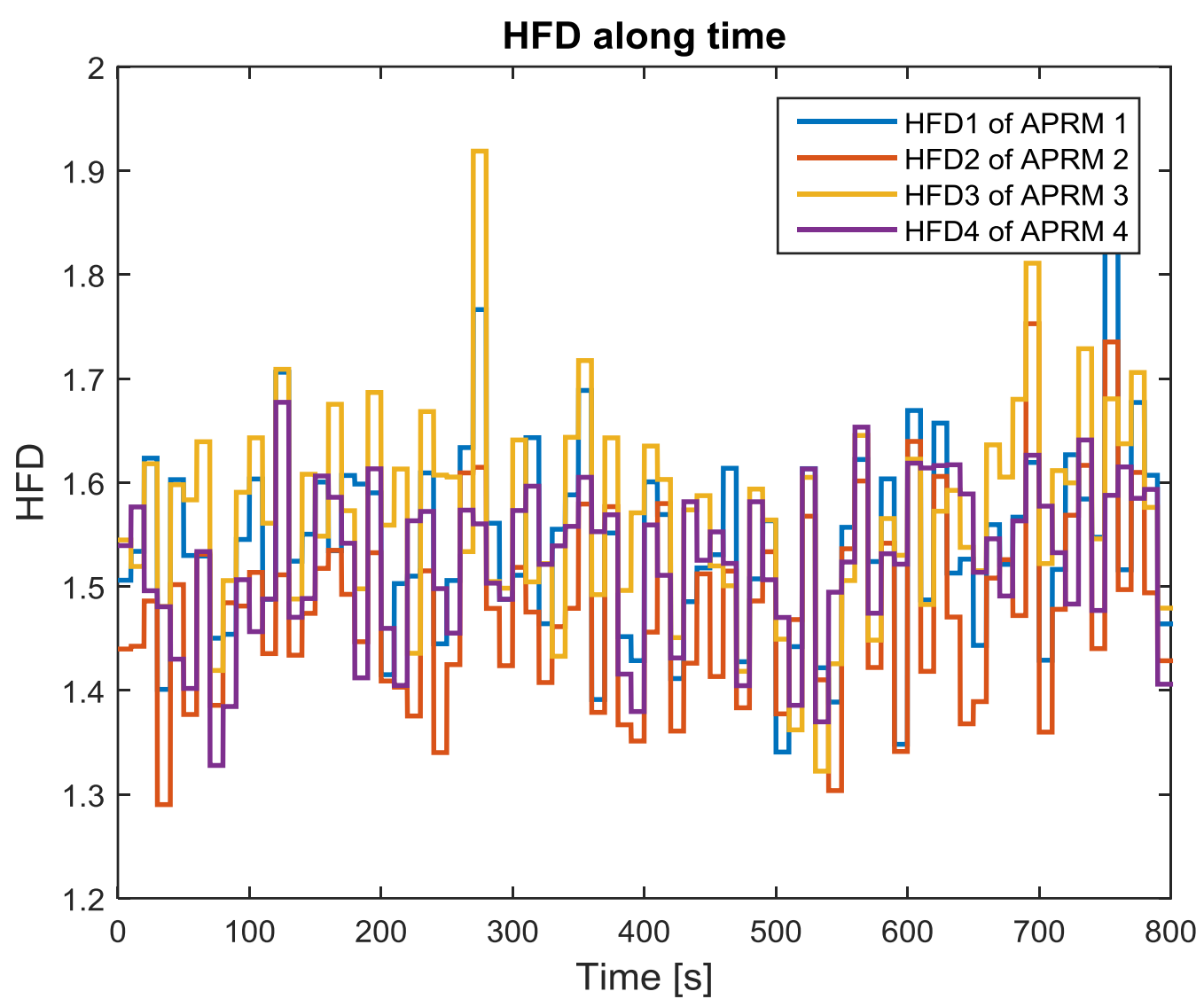

Figure 142. HFD estimates along time for the four LPRMs.

For comparisons of the proposed stability monitor (based on the HFD) with a common and well understood linear stability technique (based on the DR).

\subsection{Chapter 8 discussions}

In this chapter, a novel boiling water reactor (BWR) stability monitor was constructed with the aid of the Higuchi fractal dimension technique with the following goals:

1. Improving BWR density wave (DW) instability detection.

2. Make the BWR stability monitoring more realistic.

3. Build a methodology that can be used in conjunction with decay ratio estimation techniques that are currently the backbone of most of the BWR stability monitors out there.

4. Change to the best of our abilities the DR centered ethos of BWR stability analysis.

5. Propose a novel non-linear stability indicator rooted in fractal theory.

The proposed HFD rolling window stability monitor allowed to categorize stable BWR signals from unstable ones. The estimated HFD values of unstable cyclic density wave 
induced oscillations fluctuate close to 1 . The stability monitor was tested with signals that stem from the Ringhals stability benchmark Case 9 Cycle 14 and with signals that stem from the Forsmark stability benchmark. This monitor was also tested with stable signals that come from a typical BWR. Our goal is to deploy the monitor presented in section 3 in real time and help it to evolve accordingly. The HFD calculation needs no preprocessing of the studied time series and needs no assumptions regarding the linearity or stationarity of studied data. The HFD calculation is not rooted in the Fourier domain. The HFD is able to provide reliable data with few data points. In further research works, a phase detection module will be added to enhance this monitor capabilities in order to detect an in-phase or an out-of-phase instability event. The HFD accommodates non-linear behavior of the studied LPRM signals. 


\section{Chapter 9}

\section{Final Conclusions}

In this work, our research team decided to study BWR instability due to density waves through the lens of non-linear signal processing techniques because we believed for long that the DR was a really simple indicator to use to assess the stability of a complex dynamical system such as a BWR reactor system, besides, March-Leuba, 1986 proved 30 years ago that the BWR behavior way beyond the stability threshold might turn chaotic. It is impossible for the DR to operate under such complex unstable conditions. Our first stop was to study a signal processing technique to analyze non-stationary signals provided by non-linear systems, the empirical mode decomposition (EMD) proved to be a powerful candidate to achieve this goal, as our theoretical knowledge of the EMD grew, we understood that it was necessary to address the mode mixing problem of the default EMD method to better decompose LPRMs into IMFs. The improved CEEMDAN for uni variate signals was the selected candidate to address this problem (because it is the most recent EMD expansion to alleviate the mode mixing issue) and the NA-MEMD was selected to study simultaneously several LPRMs (by the way, The NA-MEMD can be used to analyze a uni variate signal, provided that at least two channels of white noise are added to create a multi channel signal consisting of the studied single LRPM plus the two noise channels as shown in section 3.6 at the end of Chapter 3).

Later, it was necessary to study in depth a differential equation model to understand more about the BWR behavior within its unstable region (the model had to emulate how the BWR operation is spoiled by density waves). Such simple and yet powerful ROM that we chose was developed in the late 80's by March-Leuba. By increasing one of its parameters, it was possible destabilize the reactor, and if we kept increasing such parameter, the signals provided by the ROM started to show chaotic behavior and strange attractors (Strogatz, 1994). We realized immediately that the DR might not be a suitable candidate to study such signals, to prove this belief, we tested the ROM signals under many scenarios by a conventional AR modeling of these signals to grant DR estimates. Our belief was confirmed, the DR loses all credibility to measure instability as the BWR behavior becomes more unstable (and chaotic) when it's instability parameter is increased (as shown in section 4.3). In this new light, we decided to observe BWR instability with entropy formulas to measure the complexity or intricacy of the studied data. The first candidate we implemented was a simple Shannon Entropy (SE) estimator, such computer formula estimates an optimal number of bins $M$ to calculate a Histogram (which is a probability density function (PDF) estimate of the studied data) that is used to later compute the SE, the SE estimator we developed was first tested with ROM signals, the SE measures the information content generated by the chaos of the ROM (more chaos equals more information content). Later the same SE estimator was tested with real BWR signals 
provided by many popular BWR instability benchmarks (Forsmark, Ringhals and one data set from a laguna verde instability event that happened in 1994). The SE is a quantity located in the interval $0 \leq \mathrm{SE} \leq 2$. 0 for low information content data (stable states) and closer to 2 for high information content data (unstable scenarios). In many cases the SE was high (on average $\geq 0.8$ ) for the studied signals. The simple $\mathrm{SE}$ estimator that we implemented was a starting point of this research, much improvement could still be achieved.

As our theoretical knowledge of entropy measurements increased, we decided to prove the capabilities of the Sample Entropy (SampEn), SampEn is a practical computer formula that measures the complexity of a studied time series, at that time, the SampEn remained solely tested with biomedical signals with success. We decided to explore SampEn with our BWR benchmarks, the input parameters of the SampEn, $m$ and $r$ were left at their default values ( $m=2$ and $r=0.25 \mathrm{std}$ (data) ), the SampEn detected with success BWR unstable scenarios, at the expense of longer time spans of data ( a minimum of $60 \mathrm{~s}$ of data were required to provide reliable SampEn computations). The SampEn was able to classify signals into two categories: stable signals and unstable ones (a feature that existing DR estimation methods in the literature do not provide). By the time we tested the SampEn, we had in our power for the very first time, stable signals of the laguna verde reactor that were used in the testing of the SampEn. Still, a single problem remained with this indicator, the long time spans of the studied signals.

The final indicator we tested was a fractal dimension estimator developed by Higuchi, we now measured roughness (or fractured behavior) of the studied LPRM signals. The Higuchi fractal dimension (HFD) estimator was able to provide accurate estimates with very few data points (now the minimum time span of the studied signals was of $10 \mathrm{~s}$ ) under noisy conditions ! with a really simple and reliable computer algorithm. With the HFD we were able again to classify signals into two categories: stable BWR signals and unstable BWR signals. With this HFD estimator we developed a practical and reliable BWR STABILITY MONITOR with decision rules for real time implementation (read chapter 8). Such STABILITY MONITOR based on the HFD does not need pre processing of the studied signals, it works with few data points, and the fractal dimension ignores any assumptions related to the stationary and linearity of studied data (whereas the DR needs such assumptions). This monitor differentiates stable BWR signals, known by the technical name of broad band non-coherent noise signals (BBNCN) from unstable BWR signals affected by density waves, known by the technical name of unstable cyclic oscillations. The HFD is a quantity located within the interval $[1,2]$. It is close to 1 for unstable cyclic oscillations and close to 1.5 for BBNCN signals. According to the works presented in this thesis, no stability monitor proposed in the literature is able to differentiate BBNCN signals from unstable ones. 


\subsection{Future works}

According to our own views, we could add a phase detection monitor between LPRMs to the STABILITY MONITOR proposed in chapter 8 to detect a possible out-of-phase instability event or an in-phase one. We need to test the proposed stability monitor with more BWR signals, signals that we do not have at the time and are really difficult to get. With more signals, we could obtain more information not considered at the time of the elaboration of this thesis. Another future goal would be to elaborate a patent of the stability monitor based on the HFD given in chapter 8. The estimation of the instantaneous frequencies of the IMFs related to instability can be improved as soon as more powerful Hilbert transform estimation methods are developed in the future (to improve the accuracy of the HHT step).

It would be a viable project to implement our stability methodologies in real time with the aid of FPGAs or Raspberries.

\section{References}

Akaike, H., 1974. A new look at the statistical model identification. IEEE Trans, Autom. Control 19 (6), 716-723.

Alimuradov, A.K., Tychkov, A. Y., Kuzmin, A.V., Churakov, P.P., Ageykin, A.V., Vishnevskaya, G.V., 2019. Improved CEEMDAN Based Speech Signal Analysis Algorithm for Mental Disorders Diagnostic System: Pitch Frequency Detection and Measurement. International Journal of Embedded and Real-Time Communication Systems (IJERTCS), 10(1), 22-47.

Andersen, J.G.M., et al., 1983. BWR Refill-Reflood Program Task 4.7 - Model Development, basic Models for the BWR Version of TRAC, NUREG/CR-2573, EPRI NP2375, GEAP-22051.

Anegawa, T., Ishikawa, M., Oguma, R., Lorenzen, J., 1996. Study of BWR stability at Kashiwazaki BWR unit 4 using the on-line stability monitor simon-64. In SMORN-VII proceedings, Avignon, France, Vol 1, paper 2.3.

Benedetto, J.J., 1996. Harmonic analysis and applications, Vol. 23, CRC Press. 
Blazquez, J., Ruiz, J., 2003. The Laguna Verde BWR/5 instability Event. Prog. Nucl. Energy 2003, 43, 195-200.

Bouré, J.A., 1966. The Oscillator Behavior of Heated Channels, Part I and II, French Report CEA-R 3049, Grenoble.

Bouré, J.A., Ihaila, A., 1967. The Oscillator Behavior of Heated Channels, EURATOM Report, Proc. Symp. on Two-Phase Flow Dynamics, Eindhoven.

Castillo-Durán, R., Ortiz-Servin, J. J., Castillo, A., Montes-Tadeo, J. L., Perusquía-delCueto, R., 2016. A stability assessment of optimum Fuel Reload Patterns for a BWR. Annals of Nuclear Energy 94, 841-847.

Chaiko, M.A., et al., 1990. An Analysis of Reactor Transient Response of BWR ATWS Events, Proceedings International Workshop on BWR Stability, OECD-NEA CSNI Report $178,457-476$.

Chan, K.C., 1989. FABLE02V User's Manual, NEDE-31732P.

Chon, K., Scully, C., Lu, S., 2009. Approximate entropy for all signals, IEEE Engineering in Medicine and Biology Magazine, vol. 28, no. 6, pp. 18-23.

Colominas, M.A., Schlotthauer, G., Torres, M.E., 2014. Improved complete ensemble EMD: a suitable tool for biomedical signal processing. Biomed. Signal Process. Control 14, 19-29.

Colominas, M.A., Schlotthauer, G., Torres, M.E., Flandrin, P., 2012. Noise assited EMD methods in action. Adv. Adapt. Data Anal. 4, 1250025.

Coughlin, K., Tung, K.K., 2014. Empirical mode decomposition and climate variability. In Hilbert-Huang Transform and its Applications, pp. 223-239.

Covington, L.J., Noël, A., 2003. GARDEL-BWR: Advanced BWR Online Monitoring: In Advances in Nuclear Fuel Management III. American Nuclear Society, South Carolina, USA. 
D'Auria, F., 2008. The BWR stability issue. THICKET 2008 (Seminar On the Transfer Of Competence, Knowledge And Experience Gained Through CSNI Activities in the Field of Thermal-Hydraulics).

Demazière, C., Marcel, C., Rohde, M., Van Der Hagen, T.H.J.J., 2008. Multifractal analysis of chaotic flashing-induced instabilities in boiling channels in the naturalcirculation CIRCUS facility, Nuclear Science and Engineering, 158(2), pp. 164-193.

Demeshko, M., Dokhane, A., Washio, T., Ferroukhi, H., Kawahara, Y., Aguirre, C., 2015. Application of Continuous and Structural ARMA modeling for noise analysis of a BWR coupled core and plant instability event. Annals of Nuclear Energy 75, 645-657.

Dieker, T., 2004. Simulation of fractional Brownian motion. MSc theses, University of Twente, Amsterdam, The Netherlands.

Dokhane, A., Hennig, D., Chawla, R., 2007. Interpretation of in-phase and out-of-phase BWR oscillations using an extended reduced order model and semi-analytical bifurcation analysis. Annals of Nuclear Energy 34 271-287.

Dykin, V., Demazière, C., Lange, C., Hennig, D., 2013. Investigation of global and regional BWR instabilities with a four heated-channel Reduced Order Model. Ann. Nucl. Energy 53, 381-400.

Energy, G. N., 1995. BWR Owners' Group Long-Term Stability Solution Licensing Methodology. NEDO-31960-A, November.

Espinosa-Paredes, G., Núñez-Carrera, A., Prieto-Guerrero, A., Ceceñas, M., 2007. Wavelet approach for analysis of neutronic power using data of Ringhals stability benchmark. Nucl. Eng. Des. 237, 1009-1015.

Espinosa-Paredes, G., Prieto-Guerrero, A., Nuñez-Carrera, A., Amador-Garcia, R., 2005. Wavelet-based method for instability analysis in boiling water reactors. Nucl. Technol. 151, 250-260. 
Farawila, Y.M., Pruitt, D.W., Smith, P.E., Sanchez, L., Fuentes, L.P., 1996. Analysis of the Laguna Verde instability event. In Proceedings of the National Heat Transfer Conference, Houston, TX, USA, 3-6 August 1996, Volume 9, pp. 198-202.

Fleron, J.F., 1994. A note on the history of the Cantor set and Cantor function. Mathematics Magazine, 67(2), 136-140.

Gómez, C., Hornero, R., 2010. Entropy and complexity analyses in Alzheimer's disease: an MEG study. The Open Biomedical Engineering Journal, 4, 223.

Gómez, C., Mediavilla, Á., Hornero, R., Abásolo, D., Fernández, A., 2009. Use of the Higuchi's fractal dimension for the analysis of MEG recordings from Alzheimer's disease patients. Medical engineering and physics, 31(3), 306-313.

Gonzalez, V.M., Amador, R., Castillo, R., 1995. Análisis del evento de oscilaciones de potencia en la CNLV: Informe Preliminar; Comisión Nacional de Seguridad Nuclear y Salvaguardias: Mexico City, Mexico, CNSNSTR-13, REVISION 0.

Grandi, G., Belblidia, L., Jönsson, C., 2011. BWR stability analyses with SIMULATE-3K benchmark against measured plant data. Progress in Nuclear Energy 53(6), 583-592.

Grassberger, P., Procaccia, I., 2004. Measuring the strangeness of strange attractors. In The Theory of Chaotic Attractors, Springer, New York, NY, 170-189.

Haga, T., et al., 1990. 3D Analysis of BWR Stability in Low Flow Conditions, Proceedings International Workshop on BWR Stability, OECD-NEA CSNI Report 178, 163-174.

Han, L., Van der Baan, M., 2013. Empirical mode decomposition for seismic timefrequency analysis. Geophysics, 78(2), O9-O19.

Hashimoto, K., Hotta, A., Takeda, T., 1997. Neutronic model for modal multichannel analysis of out-of-phase instability in boiling water reactor cores. Annals of Nuclear Energy, 24(2), 99-111.

Higuchi, T., 1988. Approach to an irregular time series on the basis of the fractal theory. Physica D: Nonlinear Phenomena, 31(2), 277-283. 
Hotta, A., Ninokata, H., 2002. Numerical study on observed decay ratio of coupled neutronic-thermal hydraulic instability in Ringhals Unit 1 under random noise excitation. J. Nucl. Sci. Technol. 39, 138-149.

Hu, W., Si, B.C., 2013. Soil water prediction based on its scale-specific control using multivariate empirical mode decomposition. Geoderma, 193, 180-188.

Huang, N.E., Shen, Z., Long, S.R., Wu, M.C., Shih, H.H., Zheng, Q., Yen, N.C., Tung, C.C., Liu, H.H., 1998. The empirical mode decomposition and the Hilbert spectrum for nonlinear and non-stationary time series analysis. Proceedings of the Royal Society of London A: Mathematical, Physical and Engineering Sciences, vol. 454. The Royal Society, pp. 903-995.

Hurst, H.E., 1951. Long term storage capacity of reservoirs. ASCE Transactions, 116(776), 770-808.

Ivan, M.C., 1999. Empirical mode decomposition based frequency attributes. In Proceedings of 69th SEG Meeting, Texas, USA, 1999.

Jensen, P.J., Galer, R.R., 1990. Analysis of Water Level Reduction During ATWS/Instability Events, Proceedings International Workshop on BWR Stability, OECDNEA CSNI Report 178, 446-456.

Jia, Y., Gu, H., Luo, Q., 2017. Sample entropy reveals an age-related reduction in the complexity of dynamic brain, Scientific Reports, vol. 7, no. 1.

Kanemoto, S., Andoh, Y., Yamamoto, F., Kitamura, N., 1985. Application of autoregressive modeling techniques in BWR noise analysis. Progress in Nuclear Energy, $15,85-93$.

Katz, M.J., George, E.B., 1985. Fractals and the analysis of growth paths. Bulletin of mathematical biology, 47(2), 273-286. 
Kennel, M.B., Brown, R., Abarbanel, H.D.I., 1992. Determining embedding dimension for phase-space reconstruction using a geometrical construction, Physical Review A: Atomic, Molecular and Optical Physics, vol. 45, no. 6, pp. 3403-3411.

Kesić, S., Nikolić, L., Savić, A. G., Petković, B., Spasić, S. Z., 2014. Ouabain modulation of snail $\mathrm{Br}$ neuron bursting activity after the exposure to $10 \mathrm{mT}$ static magnetic field revealed by Higuchi fractal dimension. Gen. Physiol. Biophys, 33, 335-344.

Kesić, S., Spasić, S., 2016. Application of Higuchi's fractal dimension from basic to clinical neurophysiology: A review. Computer Methods and Programs in Biomedicine, 133, 55-70.

Knuth, K.H., 2006. Optimal data-based binning for histograms. arXiv, arXiv:physics/0605197v1.

Kumar, S., Panigrahy, D., Sahu, P.K., 2018. Denoising of Electrocardiogram (ECG) signal by using empirical mode decomposition (EMD) with non-local mean (NLM) technique. Biocybernetics and Biomedical Engineering, 38(2), 297-312.

Lahey, R.T., Podoswski, M.Z., 1989. On the analysis of various instabilities in two-phase flow. Multiphase Science and Technology 4, 183- 371.

Lange, C., Hennig, D., Hurtado, A., 2011. An advanced reduced order model for BWR stability analyisis. Prog. Nucl. Energy 53, 139-160.

Lange, C., Hennig, D., Schulze, M., Hurtado, A., 2013. Comments on the application of bifurcation analyisis in BWR stability analysis. Progress in Nuclear Energy, 68, 1-15.

Lefvert, T., 1996. OECD/NEA Ringhals 1 Stability Benchmark. Nuclear Energy Agency: Boulogne-Billancourt, France.

Lehmann, C. R., LeFrancois, M. P., Mertz, W. R., GE, J. P., Pfefferlen, H. C., 1996. Reactor Stability Detect and Suppress Solutions Licensing Basis Methodology for Reload Applications NEDO-32465-A, August. 
Lei, Y., He, Z., Zi, Y., 2009. Application of the EEMD method to rotor fault diagnosis of rotating machinery. Mechanical Systems and Signal Processing, 23(4), 1327-1338.

Lei, Y., He, Z., Zi, Y., 2011. EEMD method and WNN for fault diagnosis of locomotive roller bearings. Expert Systems with Applicaitons, 38(6), 7334-7341.

Lei, Y., Liu, Z., Ouazri, J., Lin, J., 2017. A fault diagnosis method of rolling element bearings based on CEEMDAN. Proceedings of the Institution of Mechanical Engineers, Part C: Journal of Mechanical Engineering Science, 231(10), 1804-1815.

Li, S., Zhou, Q., Wu, S., Dai, E., 2006. Measurement of climate complexity using sample entropy, International Journal of Climatology, vol. 26, no. 15, pp. 2131-2139.

Li, X., Li, C., 2016. Improved CEEMDAN and PSO-SVR modeling for near-infrared noninvasive glucose detection. Computational and mathematical methods in medicine.

Lv, Y., Yan, R., Wang, T., Li, H., Song, G., 2018. Health degradation monitoring and early fault diagnosis of a rolling bearing based on CEEMDAN and improved MMSE. Materials, 11(6), 1009.

Mandelbrot, B.B., 1983. The fractal geometry of nature. Vol. 173. New York: WH freeman.

Mandelbrot, B.B., Hudson, R.L., 2010. The (mis) behavior of markets: a fractal view of risk, ruin and reward. Profile books.

Manera, A., Zboray, R., Van der Hagen, T.H.J.J., 2003. Assessment of linear and linear and non-linear autoregressive methods for BWR stability monitoring. Prog. Nucl. Energy 43 (14), 321-327.

Marcel, C. P., Rohde, M., Van Der Hagen, T. H. J. J., 2017. An experimental parametric study on natural circulation BWRs stability. Nuclear Engineering and Design 318, 135-146.

Marcel, C.P., 2007. Experimental an numerical stability investigations on natural circulation boiling water reactors, IOS press.

March-Leuba, J., 1984. Dynamic behavior of boiling water reactors. 
March-Leuba, J., 1986. A reduced-order model of boiling water reactor linear dynamics. Nucl. Technol. 75, 15-22.

March-Leuba, J., 1990. LAPUR Benchmark Against In-Phase and Out-of-Phase Stability Tests, NUREG/CR-5605, ORNL/TM-11621.

March-Leuba, J., 1992. Density-wave instabilities in boiling water reactors (No. NUREG/CR-6003; ORNL/TM-12130). Nuclear Regulatory Commission, Washington, DC (United States). Div. of Systems Technology; Oak Ridge National Lab., TN (United States).

March-Leuba, J., Blakeman, E.D., 1991. A mechanism for out-of-phase power instabilities in Boiling Water Reactors. Nuclear Science and Engineering 107, 173-179.

March-Leuba, J., Otaduy, P.J., 1983. A Comparison of BWR stability Measurements with calculations using the code LAPUR-IV. NUREG/CR-2998, ORNL/TM-8546, Oak Ridge National Laboratory, January.

March-Leuba, J., Rey, J., 1993. Coupled thermohydraulic-neutronic instabilities in boiling water nuclear reactors: a review of the state of the art. Nuclear Engineering and Design, 145(1-2), 97-111.

McFadden, J.H., et al., 1981. RETRAN-02- A program for Transient Thermal-Hydraulic Analysis of Complex Fluid Flow Systems, EPRI NP-1850-CCM.

Mitsutake, T., Tsunoyama, S., Namba, H., 1982. Application of autoregressive (AR) technique to BWR stability estimation. Progress in Nuclear Energy, 9.

Moberg, L., Tangen, K., 1986. Time Domain BWR Stability Analysis using the 3D Code RAMONA-3B, Trans. ENC, 3, 503-509.

Molla, M., Islam, K., Rahman, M.S., Sumi, A., Banik, P., 2006. Empirical mode decomposition analysis of climate changes with special reference to rainfall data. Discrete dynamics in Nature and Society.

Molla, M., Ismal, K., Ghosh, P.R., Hirose, K., 2011. Bivariate EMD-based data adaptive approach to the analysis of climate variability. Discrete Dynamics in Nature and Society. 
Montalvo, C., Gavilán-Moreno, C.J., García-Berrocal, A., 2017. Cofrentes nuclear power plant instability analysis using ensemble empirical mode decomposition (EEMD). Annals of Nuclear Energy, 1012, 390-396.

Montesinos, M.E., Muñoz-Cobo, J.L., Perez, C., 2003. Hilbert-Huang analysis of BWR neutron detector signals: application to DR calculation and to corrupted signal analysis. Annals of Nuclear Energy, 30(6), 715-727.

Moreno, G.G., 2016. Boiling water reactor instability analysis using attractor characteristics. Ann. Nucl. Energy, 88, 41-48.

Mowry, C. M., Nir, I., 2001. System for monitoring reactor instability and controlling reactor suppression U.S. Patent No. 6,173,026. Washington, DC: U.S. Patent and Trademark Office.

Muñoz-Cobo, J., Chiva, S., Sekhri, A., 2004. A reduced order model of BWR dynamics with subcooled boiling and modal kinetics: application to out of phase oscillations. Ann. Nucl. Energy 31, 1135-1162.

Muñoz-Cobo, J.L., Escrivá, A., Domingo, M.D., 2016. In-phase instabilities in BWR with sub-cooled boiling, direct heating, and spacers effects. Annals of Nuclear Energy 87, 671686.

Muñoz-Cobo, J.L., Verdú, G., 1991. Application of Hopf bifurcation theory and variational methods to the study of limit cycles in boiling water reactors. Ann. Nucl. Energy 18, 269302.

Mutlu, A.Y., Aviyente, S., 2011. Multivariate empirical mode decomposition for quantifying multivariate phase synchronization. EURASIP Journal on Advances in Signal Processing, 2011(1), 615717.

Muto, S., et al., 1990. Space-Dependent Analysis of BWR Core Nuclear Thermal Hydraulic Instability and Thermal Margin, Nucl. Eng. Design, 120, 227-239.

Navarro-Esbri, J., Ginestar, D., Verdu, G., 2003. Time dependence of linear stability parameters of a BWR. Prog. Nucl. Energy 43, 187-194. NEA/NSC/DOC, p.2. 
Neal, L.G., Zivi, S.M., Wright, R.W., 1967. The mechanisms of Hydrodynamic Instabilities in Boiling Channel, EURATOM Report, Proc. Symp. on Two-Phase Flow Dynamics, Eindhoven.

Niederreiter, H., 1992. Random Number Generation and Quasi-Monte Carlo Methods; Society for Industrial and Applied Mathematics: Philadelphia, PA, USA.

Nir, I., Mowry, C. M., 1996. System for monitoring and controlling nuclear reactors U.S. Patent No. 5,555,279. Washington, DC: U.S. Patent and Trademark Office.

Olvera-Guerrero, O.A., Prieto-Guerrero, A., Espinosa-Paredes, G., 2017. Decay Ratio estimation in BWRs based on the improved complete ensemble empirical mode decomposition with adaptive noise. Annals of Nuclear Energy, 102, 280-296.

Otaduy, P., 1979. Modelling the Dynamic Behavior of Large BWR Cores, Ph.D. Dissertation, Unversity of Florida.

Otaduy, P., March-Leuba, J., 1989. LAPUR User's Guide, NUREG/CR-5421.

Otero, M.M., Naranjo, A.R., Carralero, A.A., 2003. The attractor dimension determination applied to monitoring and surveillance in nuclear power plants. Progress in Nuclear Energy, 43(1-4), 389-395.

Pandey, V., Singh, S., 2017. Characterization of stability limits of Ledinegg instability and density wave oscillations for two-phase flow in natural circulation loops. Chemical Engineering Science 168, 204-224.

Park, C., Looney, D., Rehman, N., Ahrabian, A., Mandic, D., 2012. Classification of motor imagery BCI using multivariate empirical mode decomposition. IEEE Transactions on neural systems and rehabilitation engineering, 21(1), 10-22. 
Paruya, S., Goswami, N., Pushpavanam, S., Pillai, D. S., Bidyarani, O., 2016. Periodicallyforced density wave oscillations in boiling flow at low forcing frequencies: Nonlinear dynamics and control issues. Chemical Engineering Science 140, 123-133.

Paul, S., Singh, S., 2014. A density variant drift flux model for density wave oscillations. International Journal of Heat and Mass Transfer 69, 151-163.

Paul, S., Singh, S., 2017. On nonlinear dynamics of density wave oscillations in a channel with non-uniform axial heating. International Journal of Thermal Sciences 116, 172-198.

Peng, S.J., et al., 1985. NUFREQ-NP: A Digital Computer Code for the Linear Stability Analysis of Boiling Water Nuclear Reactors, NUREG/CR-4116.

Poza, J., Gómez, C., Bachiller, A., Hornero, R., 2012. Spectral and Non-Linear Analyses of Spontaneous Magnetoencephalographic Activity in Alzheimer's Disease. Journal of Healthcare Engineering, 3(2), 299-322.

Prieto-Guerrero, A., Espinosa-Paredes, G., 2014. Decay ratio estimation in boiling water reactors based on the empirical mode decomposition and the Hilbert-Huang transform. Prog. Nucl. Energy 71, 122-133.

Prieto-Guerrero, A., Espinosa-Paredes, G., Laguna-Sánchez, G.A., 2015. Stability monitor for boiling water reactors based on the multivariate empirical mode decomposition. Annals of Nuclear Energy, 85, 453-460.

Rasheed, B.Q.K., Qian, B., 2004. Hurst exponent and financial market predictability. In IASTED conference on Financial Engineering and Applications (FEA 2004), 203-209.

Rehman, N., Mandic, D.P., 2009. Multivariate Empirical Mode Decomposition. Proc. R. Soc. A, 466, 1291-1302. 
Rehman, N., Mandic, D.P., 2011. Filter bank property of multivariate empirical mode decomposition. IEEE Trans. Signal Process, 59, 2421-2426.

Richman, J.S., Moorman, J.R., 2000. Physiological time-series analysis using approximate entropy and sample entropy, American Journal of Physiology-Heart and Circulatory Physiology, vol. 278, no. 6, pp. H2039-H2049.

Rohatgi, U.S., 1990. Assessment of RAMONA-3B Methodology with FRIGG Dynamic Tests, Proceedings International Workshop on BWR Stability, OECD-NEA CSNI Report $178,412-429$.

Rohde, M., Manera, C.P., Van der Hagen, T.H.J.J., Shiralkar, B., 2010. Investigating the ESBWR stability with experimental and numerical tools: a comparative study, Nuclear Engineering and Design, 240(2), 375-384.

Shannon, C.E., 1948. A mathematical theory of communication. Bell Syst. Tech. J. 27 (3), $379-423$.

Shaug, J.C., et al., 1990. TRACG Analysis of BWR Plant Stability Data, Proceedings International Workshop on BWR Stability, OECD-NEA CSNI Report 178, 354-369.

Shi, Z., Tamura, Y., Ozaki, T., 2001. Monitoring the stability of BWR oscillation by nonlinear time series modeling. Ann. Nucl. Energy 28, 953-966.

Spasić, S., Kalauzi, A., Ćulić, M., Grbić, G., \& Martać, L., 2005a. Estimation of parameter kmax in fractal analysis of rat brain activity. Annals of the New York Academy of Sciences, 1048(1), 427-429.

Spasić, S., Kalauzi, A., Grbic, G., Martac, L., Culic, M., 2005b. Fractal analysis of rat brain activity after injury. Medical and Biological Engineering and Computing, 43(3), 345-348. 
Spasić, S., Nikolić, L., Mutavdžić, D., Šaponjić, J., 2011. Independent complexity patterns in single neuron activity induced by static magnetic field. Computer Methods and Programs in Biomedicine, 104(2), 212-218.

Stenning, A.H., Veziroglu, T.N., 1965. Flow Oscillations Modes in Forced Convection Boiling, Proc. 1965 Heat Transfer and Fluid Mech. Inst., Stanford Univ. Press, 301-316.

Strogatz, S.H., 1994. Nonlinear dynamics and chaos: with applications to physics, biology, chemistry, and engineering. Cambridge, MA.

Strogatz, S.H., 2014. Nonlinear Dynamics and Chaos: With Applications to Physics, Biology, Chemistry and Engineering. Westview press, USA.

Sunde, C., Pazsit, I., 2007. Wavelet techniques for the determination of the decay ratio in boiling water reactors. Kerntechnik 72, 7-19.

Suzudo, T., 2003. Application of a nonlinear dynamical descriptor to BWR stability analyses. Progress in Nuclear Energy, 43(1-4), 217-223.

Suzudo, T., Hayashi, K., Shinohara, Y., 1993. Methodology of Nuclear Reactor Monitoring and Diagnostics Using Information Dimension Application to Power Oscillation. Journal of Nuclear Science and Technology, 30(8), 727-740.

Takigawa, Y., et al., 1987. Caorso Limit Cycle Oscillation Analysis With ThreeDimensional transient Code TOSDYN-2, Nucl. Tech, 79, 210-218.

Tambouratzis, T., Antonopoulos-Domis, M., 1999. On-line stability monitoring of BWRs using artificial neural networks. Annals of Nuclear Energy, 26, 1287-1302.

Tan, X., Chen, X., Hu, X., Ren, R., Zhou, B., Fang, Z., Xia, S., 2014. EMD-based electrocardiogram delineation for a wearable low-power ECG monitoring device. Canadian Journal of Electrical and Computer Engineering, 37(4), 212-221.

Theiler, J., 1990. Estimating fractal dimension. JOSAA, 7(6), 1055-1073. 
Torres, M.E., Colominas, M.A., Schlotthauer, G., Flandrin, P., 2011. A complete ensemble empirical mode decomposition with adaptive noise. In 2011 IEEE international conference on acoustics, speech and signal processing (ICASSP), pp. 4144-4147. IEEE.

Tsuji, M., Michishita, H., Shimazu, Y. 2005. Stability monitoring for BWR based on singular decomposition method using neutral networks. Journal of Nuclear Sciences and Technology 42(12), 1054-1066.

Tsuji, M., Nishio, K., Narita, M., Ogawa, Y., Mori, M., 1993. Stability analysis of BWRs using bifurcation theory. Journal of Nuclear Sience and Technology, 30(11), 1107-1119.

Uehiro, M., Rao, Y.F., Fukuda, K., 1996. Linear stability analysis on instabilities of inphase and out-of-phase modes in boiling water reactors. J. Nucl. Sci. Technol. 33, 628-635.

Upadhyaya, B. R., Kitamura, M., 1981. Stability Monitoring of Boiling Water Reactor by Time Series Analysis of Neutron Noise. Nucl. Sci. Eng., Vol 77, p. 480.

Valtonen, K., 1990. RAMONA-3B and TRAB Assessment Using Oscillation data from TVO-J, Proceedings International Workshop on BWR Stability, OECD-NEA CSNI Report $178,205-231$.

Van der Hagen, T. H. J. J., Zboray, R., de Kruijf, W. J. M., 2000. Questioning the use of the decay ratio in BWR stability monitoring. Annals of Nuclear Energy 27, 727-732.

Van der Hagen, T.H.J.J., 1995. Artificial neural networks versus conventional methods for boiling water reactor stability monitoring. Nucl, Technol, 109, 286-305.

Verdú, G., Ginestar, D., Muñoz-Cobo, J.L., Navarro-Esbri, J., Palomo, M.J., Lansaker, P., Conde, J.M., Recio, M., Sartori, E., 2001. Forsmark 1\&2 Stability Benchmark. Time Series Analysis Methods for Oscillations During BWR Operation. Nuclear Science: New York, NY, USA, Final Report, NEA/NSC/DOC(2001)2.

Verdú, G., Ginestar, D., Muñoz-Cobo, J.L., Navarro-Esbri, Palomo, M.J., Lansaker, P., Conde, J.M., Recio, M., Sartori, E., 2001. Forsmark 1\&2 Stability Benchmark. Time Series Analysis Methods for Oscillations During BWR Operation. Final Report, NEA/NSC/DOC(2001) 2. 
Vinai, P., Demazière, C., Dykin, V., 2014. Modelling of a self-sustained density wave oscillation and its neutronic response in a three-dimensional heterogeneous system. Annals of Nuclear Energy 67, 41-48.

Wang, T., Zhang, M., Yu, Q., Zhang, H., 2012. Comparing the applications of EMD and EEMD on time-frequency analysis of seismic signal. Journal of Applied Geophysics, 83, 29-34.

Wang, W.C., Chau, K.W., Xu, D.M., Chen, X.Y., 2015. Improving forecasting accuracy of annual runoff time series using ARMA based EEMD decomposition. Water Resources Management, 29(8), 2655-2675.

Weaver, W.L., et al., 1986. TRAC-BF1 Manual: Extension to TRAC-BD1/MOD1, NUREG/CR-4391.

Weng, B., Blanco-Velasco, M., Barner, K.E., 2006. ECG denoising based on the empirical mode decomposition. In 2006 International Conference of the IEEE Engineering in Medicine and Biology Society, pp. 1-4, IEEE.

Williams, M.M.R., 1977. Reactor Noise-SMORN-II. Progress in Nuclear Energy, Vol 1. Pergamon Press.

Williams, M.M.R., 1982. Reactor Noise-SMORN-III. Progress in Nuclear Energy, Vol 9. Pergamon Press.

Wood, A.T., Chan, G., 1994. Simulation of stationary Gaussian processes in [0,1]d. Journal of computational and graphical statistics, 3(4), 409-432.

Wu, Z., Huang, H.E., 2009. Ensemble empirical mode decomposition: a noise assisted data analysis method. Adv. Adapt. Data Anal. 1, 1-41.

Wulff, W., et al., 1984. A Description and Assessment of RAMONA-3B MOD0 cycle 4: A Computer Code with Three Dimensional Neutron Kinetics for BWR System transients, NUREG/CR-3664. 

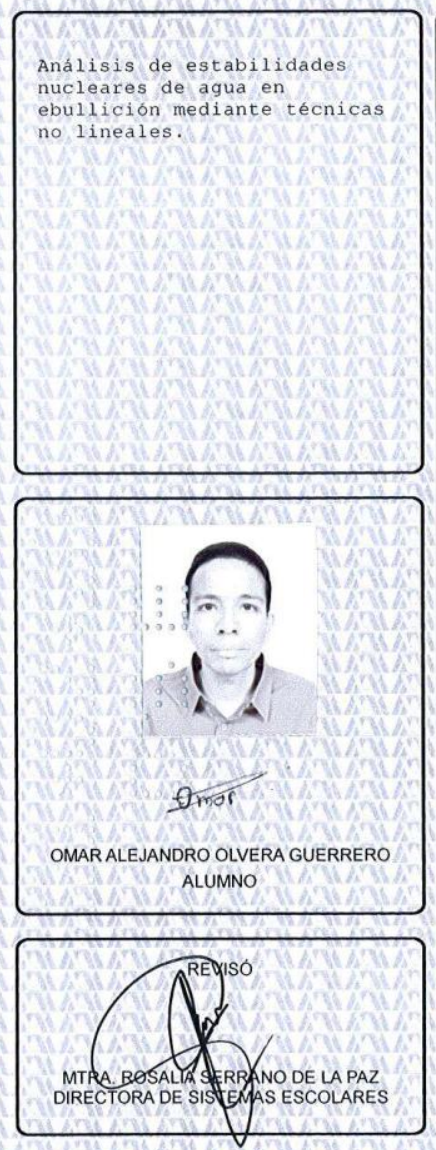

Bajo la Presidencia del primero y con caracte: de Secretario el ultimo, se reunieron a la presentación de la Disertación Publica cuya denominacion apareceral maraen. para la obtención del grado de:

DOCTOR EN CIENCIAS ICIENCIAS Y TECNOLOGIAS DE LA INFORMACION )

DE: OMAR ALEJANDRO OLVERA GUERRERO

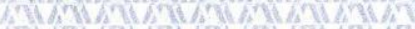

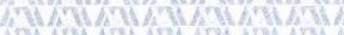

Y de acuerdo con el articulo 78 fraccion IV del Reglamento de Estudios Superiores de la universidad Autonoma Metropolitana, los miembros del jurado/resolvieron:
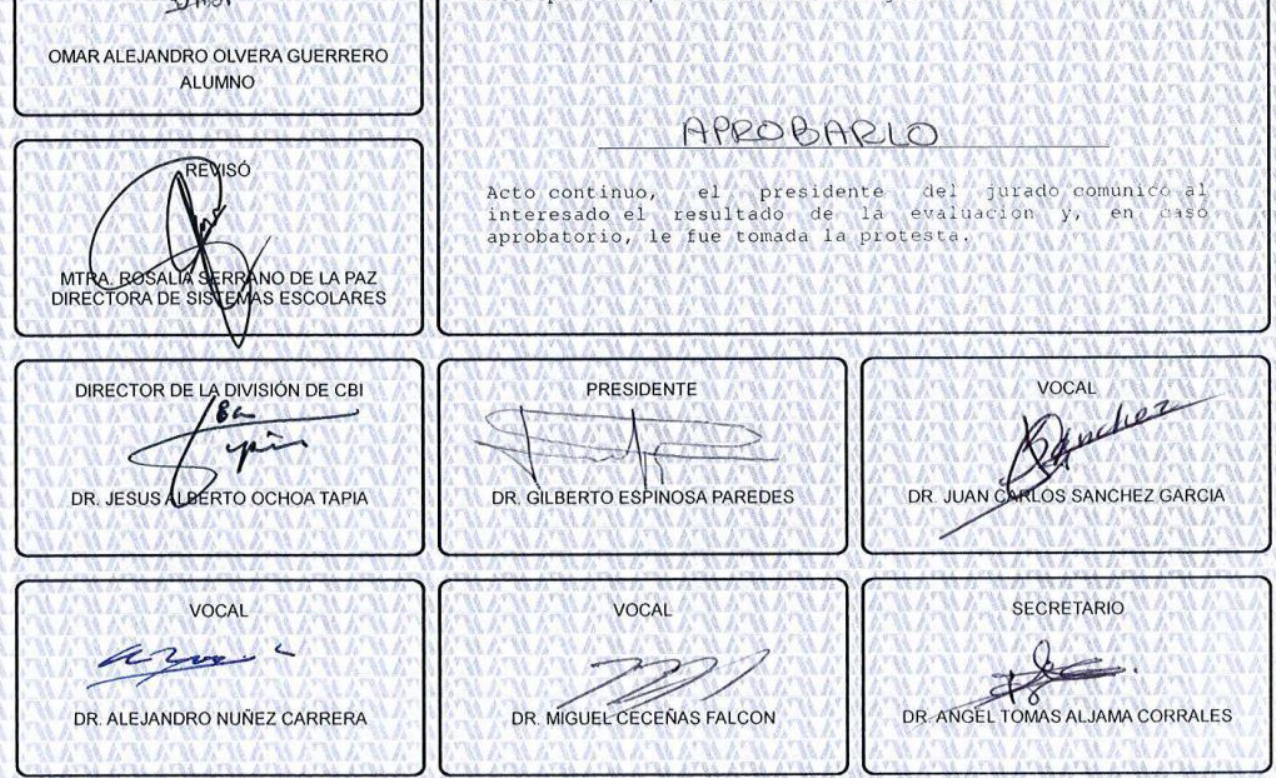


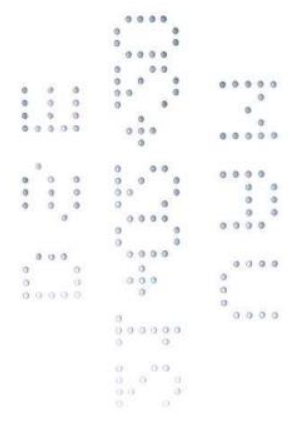

